INDIVIDUALIZED PREVENTION IN CARDIOVASCULAR DISEASE AND DIABETES MELLITUS
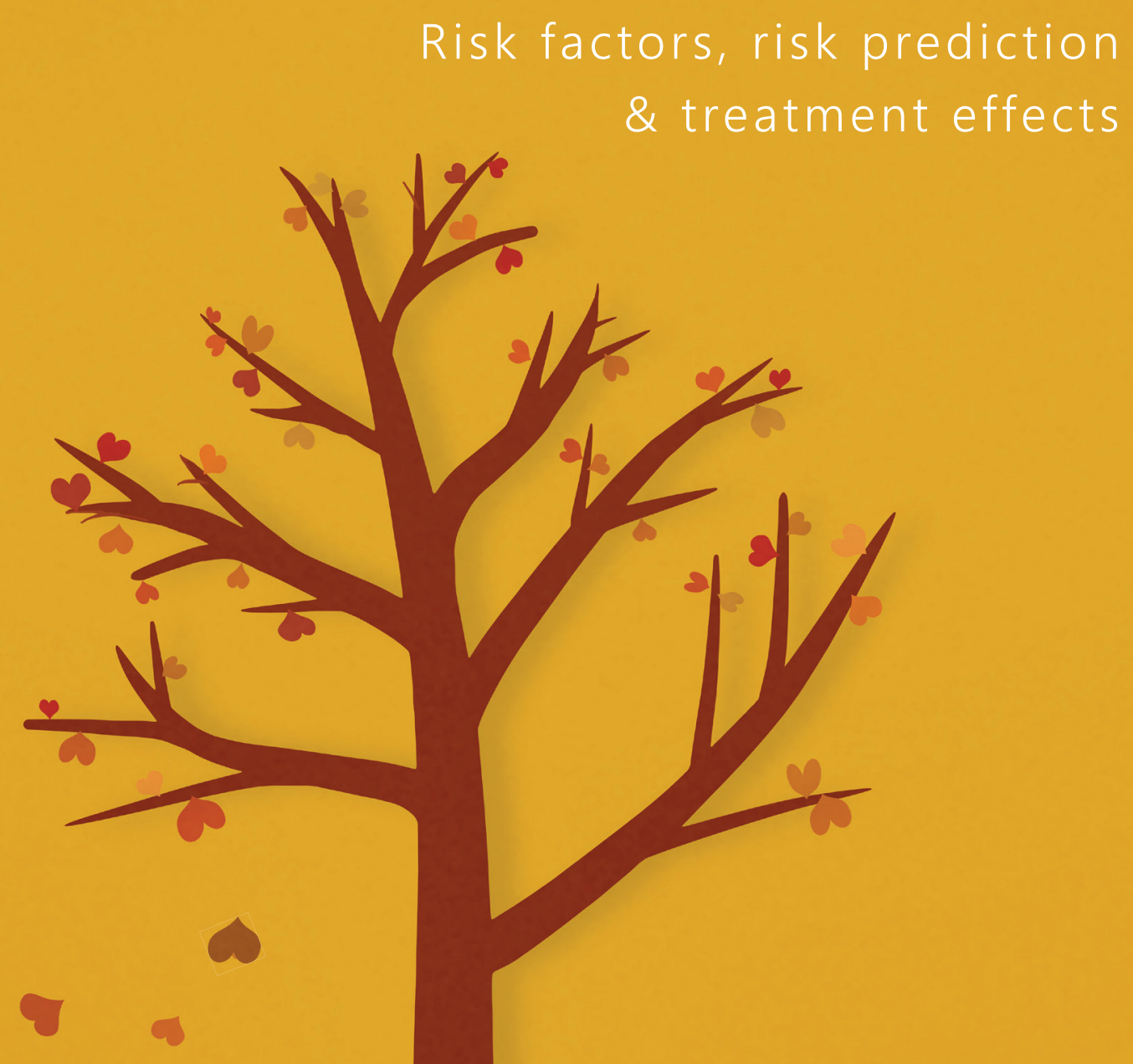

ข

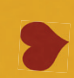

Tamar I. de Vries 



\section{Individualized prevention in cardiovascular disease and diabetes mellitus}

Risk factors, risk prediction, and treatment effects 
Individualized prevention in cardiovascular disease and diabetes mellitus: Risk factors, risk prediction, and treatment effects

Tamar de Vries

PhD thesis, department of Vascular Medicine, University Medical Center Utrecht, University Utrecht, the Netherlands

ISBN: $\quad$ 978-94-6416-160-1

Design by: Tamar I. de Vries

Printing: $\quad$ Ridderprint | www.ridderprint.nl

All rights reserved. No parts of this publication may be reproduced, stored, or transmitted, in any way or by any means, without the prior permission of the author, or when applicable, of the publishers of the scientific papers. 


\title{
Individualized prevention in cardiovascular disease and diabetes mellitus
}

Risk factors, risk prediction, and treatment effects

Geïndividualiseerde preventie van cardiovasculaire ziekte en diabetes mellitus:

risicofactoren, risicopredictie en behandeleffecten

(met een samenvatting in het Nederlands)

\begin{abstract}
Proefschrift
ter verkrijging van de graad van doctor aan de Universiteit Utrecht op gezag van de rector magnificus, prof.dr. H.R.B.M. Kummeling, ingevolge het besluit van het college door promoties in het openbaar te verdedigen op donderdag 5 november 2020 des middags te 2.30 uur
\end{abstract}

door

Tamar Irene de Vries

geboren op 3 april 1992 te Wageningen 
Promotor: $\quad$ Prof. dr. F.L.J. Visseren

Copromotor: Dr. J. Westerink

Financial support by the Dutch Heart Foundation for the publication of this thesis is gratefully acknowledged. 



\section{Table of Contents}

Chapter 1 General introduction 9

\section{Part 1. Risk factors for type 2 diabetes and cardiovascular disease}

Chapter 2 The relationship between classical vascular risk factors and cumulative recurrent cardiovascular event burden in patients with clinically manifest vascular disease: results from the UCC-SMART prospective cohort study

In revision.

Chapter 3 Thyroid-stimulating hormone levels in the normal range and incident type 2 diabetes mellitus

Acta Diabetol. 2019;56(4):431-440.

Chapter 4 Normal-range thyroid-stimulating hormone levels and cardiovascular events and mortality in type 2 diabetes Diabetes Res Clin Pract. 2019;157:107880.

\section{Part 2. Individualized prediction of treatment effects}

Chapter 5 Impact of a patient's baseline risk on the relative benefit and harm of a preventive treatment strategy: applying trial results in clinical decision-making In revision.

Chapter 6 Estimating absolute cardiovascular disease risk in individual persons aged 65 years or older using an older person-specific risk score Manuscript draft.

Chapter 7 Lifetime benefit of cholesterol-lowering, blood pressure-lowering, and smoking cessation in people without cardiovascular disease: a changing paradigm in cardiovascular disease prevention Manuscript draft. 
Chapter $8 \quad$ Estimating individual lifetime benefit and bleeding risk of adding

rivaroxaban to aspirin for patients with stable cardiovascular disease: results from the COMPASS trial

Eur Heart J. 2019;40(46):3771-3778a.

Chapter 9 Heterogeneity of treatment effects from an intensive lifestyle 189 weight loss intervention on cardiovascular events in patients with type 2 diabetes: data from the Look AHEAD trial.

Diabetes Care. 2019;42(10):1988-1994.

Chapter 10 General Discussion

\section{Chapter 11 Appendix}

Samenvatting (voor niet-ingewijden)

List of publications

Contributing authors

Dankwoord

Curriculum Vitae 


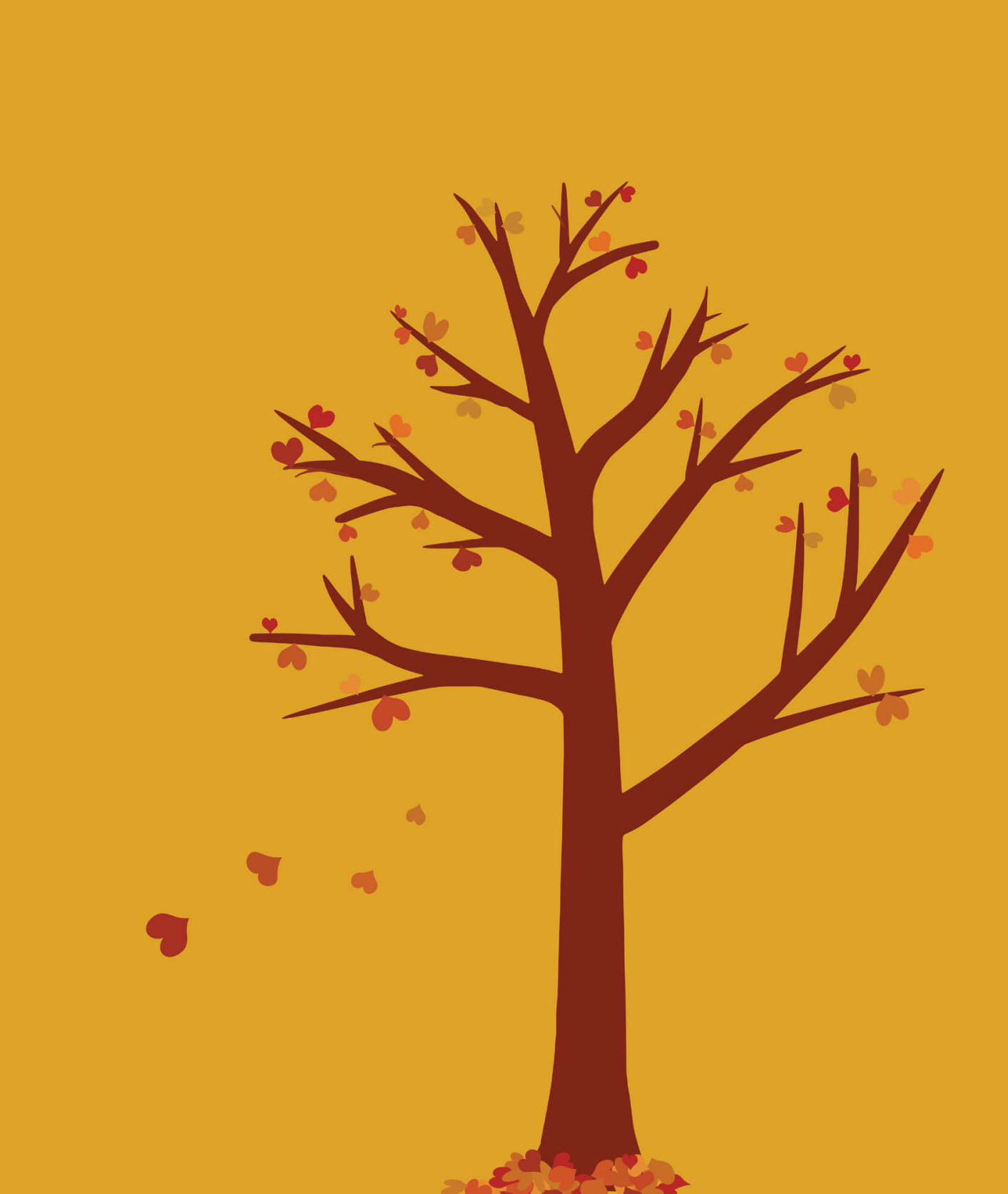




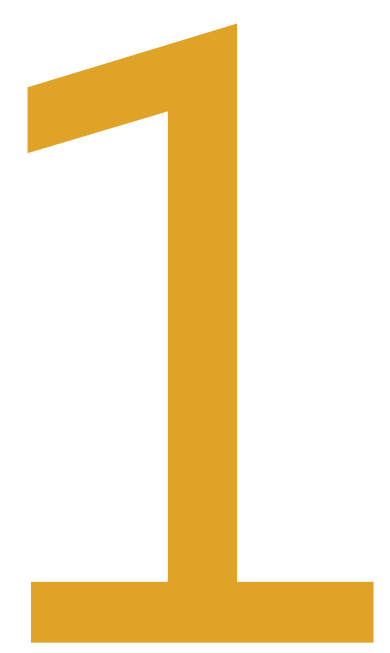

General Introduction 
In William Shakespeare's famous play Macbeth, Lord Banquo speaks to the Three Witches: "If you can look into the seeds of time, and say which grain will grow and which will not, speak then unto me." As physicians, we too wish we could look into the future and know with certainty which of our patients are going to suffer from disease and which are not. In a patient not at risk of a given disease, we can safely withhold preventive strategies, thereby sparing the patient burden of treatment, costs, and potential adverse events. And in a patient who is going to develop a given disease, we can intensify or commence treatment to delay onset, or even to prevent disease from occurring. Being able to look into the future would thus help immensely in daily clinical practice. Unfortunately, no Shakespearean witches are available for foretell the future of our patients. Therefore, in clinical practice we have to rely on different methods to foresee their future.

\section{Is my patient at risk for cardiovascular disease?}

Clinical intuition alone may tell us that a certain patient is at high risk of developing cardiovascular disease (CVD). A textbook patient at high risk of CVD is a middle-aged male, who smokes, and who suffers from diabetes, dyslipidemia, and hypertension. Based on clinical experience, his doctor will very likely consider this individual at high risk of future CVD, and recommend him to stop smoking and initiate treatment to lower his blood glucose, lipids, and blood pressure. However, there are many patients for whom preventive treatment choices are not so clear-cut. For example, what of a patient who has never smoked, lives generally healthy, and has a normal blood pressure, but whose lowdensity lipoprotein cholesterol is too high? Is it really necessary to initiate lipid-lowering therapy in such a patient? And what of an older patient who has hypertension, but who is afraid of adverse effects from blood pressure lowering therapy? Could we defend the decision not to initiate blood pressure-lowering therapy in this patient? Or should we perhaps treat all patients, as most patients who develop their first cardiovascular event were at a predicted low short term risk before the event? These decisions can be difficult to make in clinical practice on the basis of clinical intuition alone.

\section{Risk factors for cardiovascular disease}

To even begin knowing which patients are at high risk of developing CVD, we first need to know what risk factors are important for the development of CVD. Many risk factors are already widely known from etiologic studies, with high blood pressure, ${ }^{1}$ dyslipidemia, ${ }^{2}$ diabetes, ${ }^{3}$ and smoking ${ }^{4}$ being considered as some the most important amendable risk factors for developing CVD, but also for the risk of a recurrent CVD event in patients with established vascular disease. ${ }^{5}$ 
However, even with optimal control of these 'traditional' risk factors there is still a residual risk for CVD, especially in so-called 'high-risk' patients such as patients with established CVD or with type 2 diabetes. ${ }^{6,7}$ Therefore, there is an ongoing interest to discover more, potentially treatable, risk factors for CVD. Characterizing other potentially causal factors for the residual risk of CVD may lead to new pathophysiological insights and, consequently, possibly to future strategies aimed at further reducing the residual CVD risk.

One of these risk factors under study is thyroid function. The cardiovascular system is directly influenced by thyroid hormones, which have effects on vascular smooth muscle cell relaxation, vascular resistance, cardiac contractility, and heart rate. ${ }^{8}$ Thyroid function is also associated with classical cardiovascular risk factors such as lipid levels, blood pressure, and adiposity, ${ }^{9-12}$ and additionally, there is evidence from previous literature suggests that thyroid function, even within the normal range, is associated with the risk of type 2 diabetes.13,14 Understanding the role of new risk factors, such as thyroid function, in the pathophysiology of diabetes mellitus and CVD might provide new leads and opportunities for future research to fully understand the occurrence of these important chronic diseases.

\section{Identifying patients at high risk of cardiovascular disease}

Just knowing which risk factors are associated with diabetes and CVD is not enough to know which patients is at high risk of CVD, as it is usually a combination of risk factors in an individual patient that determines their risk of future events. Incorrectly estimating the prognosis of a patient may lead to unnecessary treatment in low risk patients or undertreatment in patients at high risk.

In current international guidelines, there are evidence-based recommendations for making these treatment decisions, based on a combination of risk factor levels together with the future risk of cardiovascular events. ${ }^{15,16}$ Knowing which patients will benefit from cardiovascular risk management by treatment of risk factors is important for all possible treatment options, from lifestyle, to lipid and blood pressure lowering, to the use of antithrombotic medication, as all these therapies are also associated with a patient burden, costs, and especially in the latter case, the risk of adverse (bleeding) events.

\section{Risk stratification in current clinical practice}

In the current guidelines, patients with established CVD are deemed to be at such a high risk of future CVD that pharmacotherapy is almost always warranted. 15,16 Patients with type 2 diabetes, especially those with target organ damage or several major risk factors, are also deemed at (very) high risk. In the primary prevention setting, risk stratification using multivariate risk models is advised to predict an individual patient's future risk of developing CVD. The Systematic COronary Risk Estimation (SCORE) model to estimate fatal 
CVD is recommended in the European Society of Cardiology/European Atherosclerosis Society (ESC/EAS) guideline, ${ }^{15}$ and the pooled cohort equation to estimate the risk of atherosclerotic CVD (ASCVD) is recommended in the American College of Cardiology/ American Heart Association (ACC/AHA) guidelines. ${ }^{16}$ Both these risk scores estimate the 10 -year risk of CVD. Above a certain threshold, patients qualify for pharmacotherapy for lipid- and blood pressure-lowering. ${ }^{17}$

Risk stratification using individualized risk estimation is not recommended in the current 2016 European CVD prevention guidelines for patients with type 2 diabetes or established $C V D$, as they are all deemed to be at high or very high risk of future CVD events. However, in the 2019 Dutch Cardiovascular Risk Management guideline the option is presented to calculate (residual) CVD risk in patients with established CVD as well as in patients with type 2 diabetes. ${ }^{18}$ It has been shown that patients with diabetes or established CVD are not all at equally high risk for (recurrent) CVD. ${ }^{6,19}$ Therefore, clinical decision making in these patients could be improved by using individualized risk prediction for a more personalized approach to (recurrent) CVD prevention.

\section{Individualized risk prediction}

Unfortunately, individual risk prediction is not easy. As Peter McCullagh and John Nelder wrote in their book about generalized linear models: "All models are wrong; some, though, are better than others and we can search for the better ones." 20 And it is indeed very necessary to search for those better models, as more than 350 models - and counting - have been developed for the prediction of CVD risk in the general population alone. ${ }^{21}$ The majority of these models will never be used in clinical practice, due to lack of external validation, the use of predictors that are not usually available in daily practice, methodological limitations, and incomplete presentation. Focus in research should therefore be on optimization and clinical implementation of existing, easy-to-use risk models rather than the development of new highly specific risk models using predictors that are not routinely measured in clinical practice. However, for some patient populations or clinical outcomes, no (adequate) models yet exist. In that case, it can still prove to be useful to develop new risk models.

\section{Disadvantages of 10-year CVD risk models}

As mentioned previously, several 10-year CVD risk models currently have a place in international guidelines. However, 10-year risks are mostly driven by patient age. This means that young patients are almost invariably at low risk of CVD according to these models, and thus usually do not have an indication for preventive therapy. However, 
younger patients with a high burden of risk factors may have very high lifetime risk of developing CVD. As atherosclerosis is a result of lifelong exposure to risk factors, it makes sense to start therapy at young age to stop or slow down the atherosclerotic process early. Traditional 10-year risk scores cannot be used for this purpose as they would only advice treatment in older patients.

At the same time, traditional 10-year risk models such as Framingham and SCORE also overestimate the 10-year risk of CVD in older patients, as they do not take the risk of non-CVD mortality - so-called "competing events" - into account. Furthermore, the relationship between classical risk factors such as lipids and blood pressure with CVD risk attenuates with age. ${ }^{22}$ Therefore, a competing risk-adjusted, older person-specific risk score should be used in older patients. ${ }^{23}$

\section{From CVD risk to treatment benefit}

The underlying idea of risk stratification in the current guidelines is that the greater the baseline risk, the greater the potential benefit from preventive therapy. To quantify this potential, the number needed to treat (NNT) is often used in discussions, although the temporal aspect of NNT (for a specific number of years) is often left out. As using risk stratification helps in selecting those patients who likely have the highest risk, it is a way to try to optimize the balance between the advantages from preventive treatment versus potential disadvantages, such as adverse events and costs. This is necessary to find the right balance between overtreatment, with risk of adverse events and potential costs, and under-treatment, which may lead to cardiovascular events that could have been prevented. Thus, risk predictions tools can help make informed treatment decisions for cardiovascular risk management, and can be used to inform patients about their prognosis.

A more recent development in preventive medicine is to look at the potential treatment benefit directly, rather than the underlying risk as a proxy of benefit. It is possible to predict treatment effects for individual patients by combining estimations of future CVD risk with hazard ratios from trials or meta-analyses, ${ }^{24}$ under the assumption that although absolute risk may differ greatly, the relative treatment effect remains the same for all patients. ${ }^{25}$

The clinical implementation of risk calculators that provide estimates of risk factor modification is already recommended by the "ESC Prevention of Cardiovascular Disease Programme" led by the European Association of Preventive Cardiology for use in different patient populations, such as healthy individuals, patients with established CVD, patients with diabetes mellitus, and older persons. ${ }^{26}$ Alternatively, it is possible to directly estimate individual treatment effects in trials using multivariate models that include 
treatment allocation-by-covariate interaction terms. ${ }^{25}$ This methodology allows for truly individualized treatment effect estimations without assuming a homogenous relative treatment effect.

\section{Higher risk equals higher benefit?}

It was stated in the previous paragraph that "higher risk leads to a higher potential benefit". While this is undoubtedly true, there is not just one way to determine what it means to be 'at high risk'. Until now we have discussed classical risk scores estimating the 10 -year risk of CVD using multivariate risk models, which together with the hazard ratios from trials can be used to estimate absolute 10-year risk reductions. However, we have stated before that there are potential limitations to the 10 -year risk models currently used in clinical practice.

Recent methodological advances allow us to look at future event risk in a life-long perspective, using lifetime prediction models. These models express treatment effects in terms of "months (or years) of event-free life expectancy gained or lost", or in a lifetime risk reduction. ${ }^{27}$ This is a relatively new metric which is more appealing to use in patient communication compared to risk reductions, which are presented in percentages. ${ }^{28,29}$

Figure 1 shows an example of how we can estimate the treatment effect of $10 \mathrm{mmHg}$ systolic blood pressure reduction in two patients using both a 10-year and lifetime perspective. Using the 10-year risk reduction, patient B seems to benefit more from blood pressure lowering than patient $A$. However, patient B, considered at very-high risk due to his high 10-year risk, has a markedly lower lifetime benefit than patient $A$. While patient $A$ is considered at low risk, and thus SBP reduction would not be warranted under the current guidelines, their lifetime benefit is substantial.

Compared to a risk-based approach, lifetime treatment effect estimations therefore have the potential to shift the focus from treating those with the highest risk of CVD to treating those with the highest potential lifetime benefit, which may be very different patients. ${ }^{30}$ Several lifetime prediction models are available online to predict lifetime treatment effects from medications, including JBS-3 and LIFE-CVD for primary prevention, ${ }^{30,31}$ SMART-REACH for patients with established CVD, ${ }^{32}$ and DIAL for patients with type 2 diabetes. ${ }^{33}$

\section{Preventive strategies: finding the balance}

Important to realize is that an individual person is not truly at $10 \%$ or $20 \%$ or $50 \%$ risk of having a cardiovascular event. An individual is during their life either at $0 \%$ risk (they will not experience an event) or at 100\% risk (they will experience an event). Reliable risk estimations are useful for making treatment decisions, but the estimates are only true 
Figure 1: Patient example

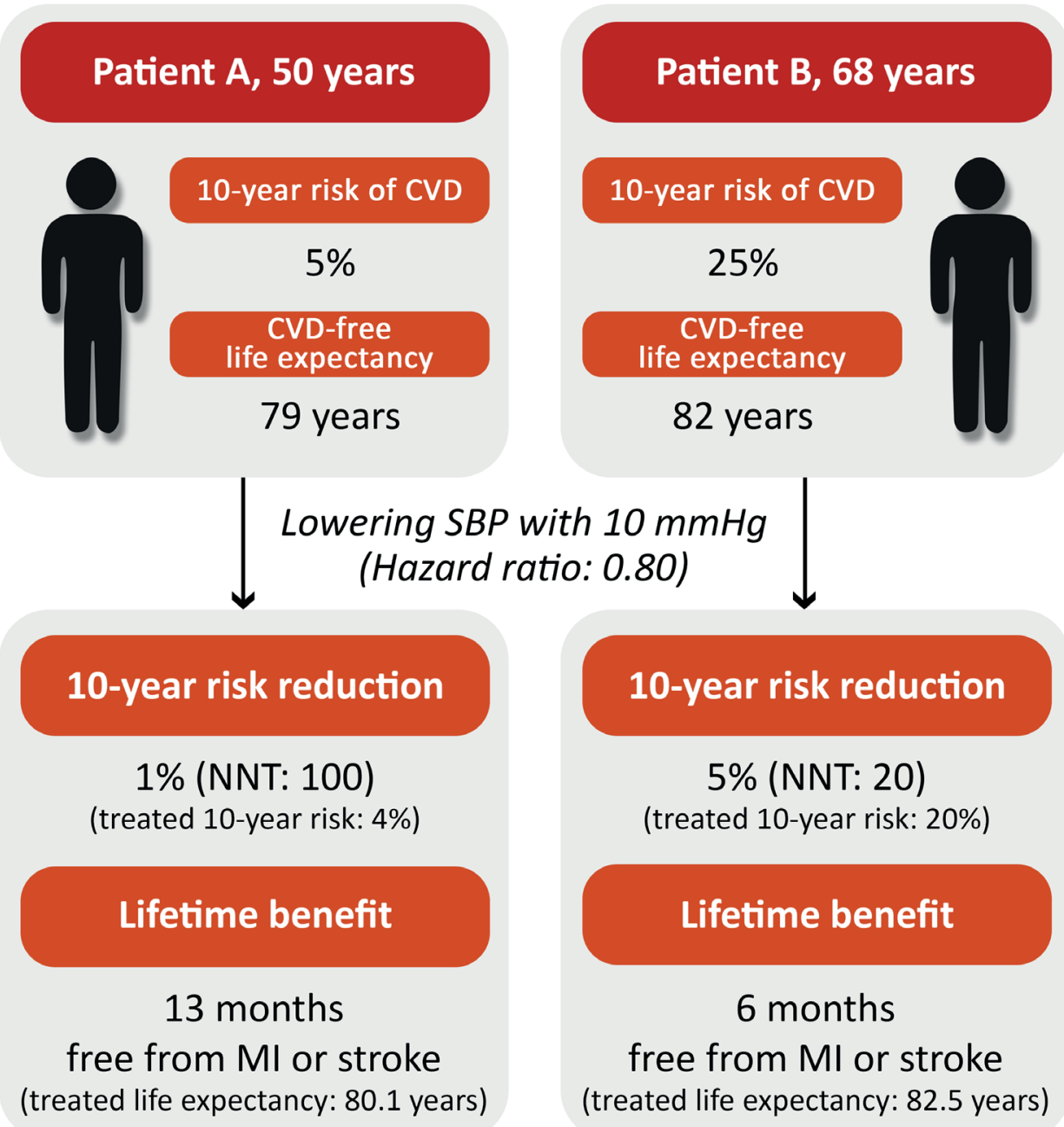

CVD = cardiovascular disease; $S B P=$ systolic blood pressure; $\mathrm{MI}=$ myocardial infarction; NNT = number needed to treat

at a group level. When making treatment decisions in CVD prevention, we always need to weigh risks - after all, when treating a patient with preventive pharmacotherapy, the patient is also at risk for adverse events. Therefore, discussions about cardiovascular risk management should always include the weighing of both benefits and disadvantages. The lower the risk of CVD at baseline, the lower the potential treatment benefit - and thus, the higher the chance that the benefits do not outweigh the disadvantages. This is especially true when treatment is burdensome, costly, or has an important risk of adverse events. The latter may for example be important in case of antithrombotic use, with a risk 
of major bleeding. ${ }^{34,35}$ It may therefore be very useful to not only predict the treatment effects of preventive medication on the risk of CVD, but also on the risk of important adverse effects.

Individual risk estimations, CVD-free life expectancy, and treatment effect estimations can thus be used in clinical practice for shared-decision making between clinicians and patients to choose the right therapy for the right patient. 


\section{Thesis objectives}

The general objective of this thesis are (1) to investigate potential risk factors for the development of cardiovascular disease and type 2 diabetes, and (2) to predict cardiovascular risk and treatment effects of preventive therapy for individual patients.

\section{Thesis outline}

Part 1 focuses on risk factors of the development of cardiovascular disease and diabetes mellitus. In Chapter 2, the relationship between classical risk factors for cardiovascular disease and the risk of all recurrent cardiovascular events, the total cumulative disease burden, is investigated. In Chapter 3, the relationship between thyroid-stimulating hormone in the normal range and the risk of incident type 2 diabetes is examined in patients at high risk of cardiovascular disease from the SMART cohort study. In Chapter 4, the relationship between thyroid-stimulating hormone in the normal range and the risk of cardiovascular disease and mortality is examined in patients with type 2 diabetes from the SMART cohort study.

Part 2 of this thesis focuses on the prediction of cardiovascular risk and treatment effects of preventive medication in individual patients. In Chapter 5, the assessment of treatment effect modification by baseline event risk is illustrated using two major contemporary trials. Effect modification by baseline risk using multivariate risk assessment should be performed routinely in clinical trials. Data from trials provide the opportunity to estimate treatment effects for individual patients from different patient populations. In Chapter 6, an older-person specific risk score is validated for the prediction of CVD risk in patients aged over 65 years, and used for the estimation of the treatment effects of blood pressure lowering. Chapter 7 assesses the differences in treatment benefit from risk factor management from an absolute risk reduction perspective compared to a lifetime perspective. In Chapter 8, two lifetime models are presented to estimate the lifetime treatment effect from adding rivaroxaban to aspirin in patients with stable cardiovascular disease. These treatment effects are expressed in both life-years free of stroke and myocardial infarction gained, and life-years free from major bleeding lost. Chapter 9 focuses on the direct estimation of treatment effects from an intensive lifestyle intervention aimed at weight loss in overweight and obese patients with type 2 diabetes for the prevention of CVD using a multivariate approach. The main findings of this thesis are discussed in Chapter $\mathbf{1 0}$ and summarized in Chapter $\mathbf{1 1}$. 


\section{References}

1. Lewington S, Clarke R, Qizilbash N, Peto R, Collins R. Age-specific relevance of usual blood pressure to vascular mortality: a meta-analysis of individual data for one million adults in 61 prospective studies. Lancet. 2002;360(9349):1903-13.

2. Lewington S, Whitlock G, Clarke R, Sherliker P, Emberson J, Halsey J, et al. Blood cholesterol and vascular mortality by age, sex, and blood pressure: a meta-analysis of individual data from 61 prospective studies with 55,000 vascular deaths. Lancet. 2007;370(9602):1829-39.

3. Huxley R, Barzi F, Woodward M. Excess risk of fatal coronary heart disease associated with diabetes in men and women: meta-analysis of 37 prospective cohort studies. BMJ. 2006;332(7533):73-8.

4. Gakidou E, Afshin A, Abajobir AA, Abate KH, Abbafati C, Abbas KM, et al. Global, regional, and national comparative risk assessment of 84 behavioural, environmental and occupational, and metabolic risks or clusters of risks, 1990\&\#x2013;2016: a systematic analysis for the Global Burden of Disease Study 2016. Lancet. 2017;390(10100):1345-422.

5. van den Berg MJ, Westerink J, van der Graaf Y, Kappelle LJ, de Borst GJ, Cramer M-JM, et al. Risk Factors for Recurrent Cardiovascular Events Before Age 65 Years or Within 2.5 Years of a Recent First Cardiovascular Event. Am J Cardiol. 2017;120(2):167-73.

6. Kaasenbrood L, Boekholdt SM, van der Graaf Y, Ray KK, Peters RJG, Kastelein JJP, et al. Distribution of Estimated 10-Year Risk of Recurrent Vascular Events and Residual Risk in a Secondary Prevention Population. Circulation. 2016;134(19):1419-29.

7. Engelen SE, van der Graaf Y, Stam-Slob MC, Grobbee DE, Cramer MJ, Kappelle LJ, et al. Incidence of cardiovascular events and vascular interventions in patients with type 2 diabetes. Int J Cardiol. 2017;248:301-7.

8. Danzi S, Klein I. Thyroid disease and the cardiovascular system. Endocrinol Metab Clin North Am. 2014;43(2):517-28.

9. Garduno-Garcia J de J, Alvirde-Garcia U, Lopez-Carrasco G, Padilla Mendoza ME, Mehta R, Arellano-Campos $\mathrm{O}$, et al. TSH and free thyroxine concentrations are associated with differing metabolic markers in euthyroid subjects. Eur J Endocrinol. 2010;163(2):273-8.

10. Zhang Y, Lu P, Zhang L, Xiao X. Association between lipids profile and thyroid parameters in euthyroid diabetic subjects: a cross-sectional study. BMC Endocr Disord. 2015;15:12.

11. Åsvold BO, Bjøro T, Nilsen TIL, Vatten LJ, Asvold BO, Bjoro T, et al. Association between Blood Pressure and Serum Thyroid-Stimulating Hormone Concentration within the Reference Range: A Population-Based Study. J Clin Endocrinol Metab. 2007;92(3):841-5.

12. Westerink J, van der Graaf Y, Faber DR, Visseren FLJ, SMART study group. The relation between thyroid-stimulating hormone and measures of adiposity in patients with manifest vascular disease. Eur J Clin Invest. 2011;41(2):159-66.

13. Gu Y, Li H, Bao X, Zhang Q, Liu L, Meng G, et al. The relationship between thyroid function and the prevalence of type 2 diabetes mellitus in euthyroid subjects. J Clin Endocrinol Metab. 
2017;102(2):434-42.

14. Chaker L, Ligthart S, Korevaar TIM, Hofman A, Franco OH, Peeters RP, et al. Thyroid function and risk of type 2 diabetes: a population-based prospective cohort study. BMC Med. 2016;14(1):150.

15. Piepoli MF, Hoes AW, Agewall S, Albus C, Brotons C, Catapano AL, et al. 2016 European guidelines on cardiovascular disease prevention in clinical practice: the Sixth Joint Task Force of the European Society of Cardiology and Other Societies on Cardiovascular Disease Prevention in Clinical Practice. Eur Heart J. 2016;37(29):2315-81.

16. Goff DCJ, Lloyd-Jones DM, Bennett G, Coady S, D'Agostino RB, Gibbons R, et al. 2013 ACC/ AHA guideline on the assessment of cardiovascular risk: a report of the American College of Cardiology/American Heart Association Task Force on Practice Guidelines. Circulation. 2014;129(25):S49-73.

17. Mach F, Baigent C, Catapano AL, Koskinas KC, Casula M, Badimon L, et al. 2019 ESC/EAS Guidelines for the management of dyslipidaemias: lipid modification to reduce cardiovascular risk: The Task Force for the management of dyslipidaemias of the European Society of Cardiology (ESC) and European Atherosclerosis Society (EAS). Eur Heart J. 2020 1;41(1):111-188.

18. NHG/NIV/NVVC. Multidisciplinaire richtlijn Cardiovasculair risicomanagement. Utrecht: NHG/ NIV/NVVC; 2018.

19. Kaasenbrood L, Poulter NR, Sever PS, Colhoun HM, Livingstone SJ, Boekholdt SM, et al. Development and Validation of a Model to Predict Absolute Vascular Risk Reduction by Moderate-Intensity Statin Therapy in Individual Patients With Type 2 Diabetes Mellitus: The Anglo Scandinavian Cardiac Outcomes Trial, Antihypertensive and Lipid-Lowering. Circ Cardiovasc Qual Outcomes. 2016;9(3):213-21.

20. McCullagh P, Nelder JA. Generalized Linear Models. London, United Kingdom: Chapman \& Hall; 1983.

21. Damen JAAG, Hooft L, Schuit E, Debray TPA, Collins GS, Tzoulaki I, et al. Prediction models for cardiovascular disease risk in the general population: systematic review. BMJ. 2016;353:i2416.

22. Kannel WB, D'Agostino RB. The Importance of Cardiovascular Risk Factors in the Elderly. Am J Geriatr Cardiol. 1995;4(2):10-23.

23. Stam-Slob MC, Visseren FLJ, Jukema J, van der Graaf Y, Poulter NR, Gupta A, et al. Personalized absolute benefit of statin treatment for primary or secondary prevention of vascular disease in individual elderly patients. Clin Res Cardiol. 2017;106(1):58-68.

24. Dorresteijn JAN, Visseren FLJ, Ridker PM, Wassink AMJ, Paynter NP, Steyerberg EW, et al. Estimating treatment effects for individual patients based on the results of randomised clinical trials. BMJ. 2011;343:d5888.

25. Kent DM, Steyerberg E, van Klaveren D. Personalized evidence based medicine: predictive approaches to heterogeneous treatment effects. BMJ. 2018;363:k4245.

26. Rossello X, Dorresteijn JAN, Janssen A, Lambrinou E, Scherrenberg M, Bonnefoy-Cudraz E, et al. Risk prediction tools in cardiovascular disease prevention: A report from the ESC Prevention of CVD Programme led by the European Association of Preventive Cardiology (EAPC) in 
collaboration with the Acute Cardiovascular Care Association (ACCA) and the As. Eur J Prev Cardiol. 2019;2047487319846715.

27. Dorresteijn JAN, Kaasenbrood L, Cook NR, van Kruijsdijk RCM, van der Graaf Y, Visseren FLJ, et al. How to translate clinical trial results into gain in healthy life expectancy for individual patients. BMJ. 2016;352:i1548.

28. Jaspers NEM, Visseren FLJ, Numans ME, Smulders YM, van Loenen Martinet FA, van der Graaf $Y$, et al. Variation in minimum desired cardiovascular disease-free longevity benefit from statin and antihypertensive medications: a cross-sectional study of patient and primary care physician perspectives. BMJ Open. 2018;8(5):e021309.

29. Grisolia JM, Longo A, Hutchinson G, Kee F. Comparing mortality risk reduction, life expectancy gains, and probability of achieving full life span, as alternatives for presenting CVD mortality risk reduction: A discrete choice study of framing risk and health behaviour change. Soc Sci Med. 2018 Aug;211:164-74.

30. Jaspers NEM, Ridker PM, Dorresteijn JAN, Visseren FLJ. The prediction of therapy-benefit for individual cardiovascular disease prevention: rationale, implications, and implementation. Curr Opin Lipidol. 2018 Dec;29(6):436-44.

31. Joint British Societies' consensus recommendations for the prevention of cardiovascular disease (JBS3). Heart. 2014;100(Suppl 2):ii1 LP-ii67.

32. Kaasenbrood L, Bhatt DL, Dorresteijn JAN, Wilson PWF, D'Agostino RB Sr, Massaro JM, et al. Estimated Life Expectancy Without Recurrent Cardiovascular Events in Patients With Vascular Disease: The SMART-REACH Model. J Am Heart Assoc. 2018;7(16):e009217.

33. Berkelmans GF, Gudbjörnsdottir S, Visseren FL, Wild SH, Franzen S, Chalmers J, et al. Prediction of individual life-years gained without cardiovascular events from lipid, blood pressure, glucose, and aspirin treatment based on data of more than 500000 patients with Type 2 diabetes mellitus. Eur Heart J. 2019;40(34):2899-2906

34. Collaboration AT (ATT), Baigent C, Blackwell L, Collins R, Emberson J, Godwin J, et al. Aspirin in the primary and secondary prevention of vascular disease: collaborative meta-analysis of individual participant data from randomised trials. Lancet (London, England). 2009;373(9678):1849-60.

35. Eikelboom JW, Connolly SJ, Bosch J, Dagenais GR, Hart RG, Shestakovska O, et al. Rivaroxaban with or without Aspirin in Stable Cardiovascular Disease. N Engl J Med. 2017;377(14):1319-30. 
CHAPTER 1 | General introduction 

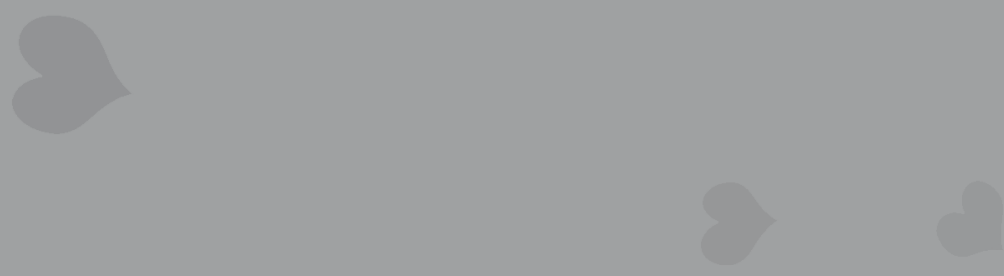

8
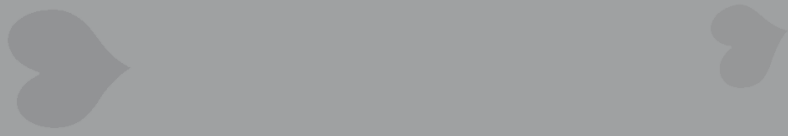


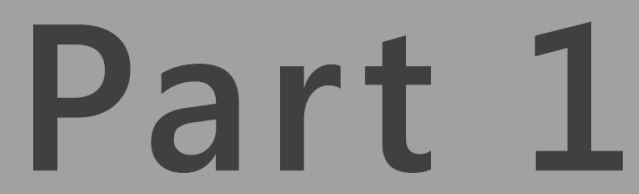

Risk factors for type 2 diabetes and cardiovascular disease 


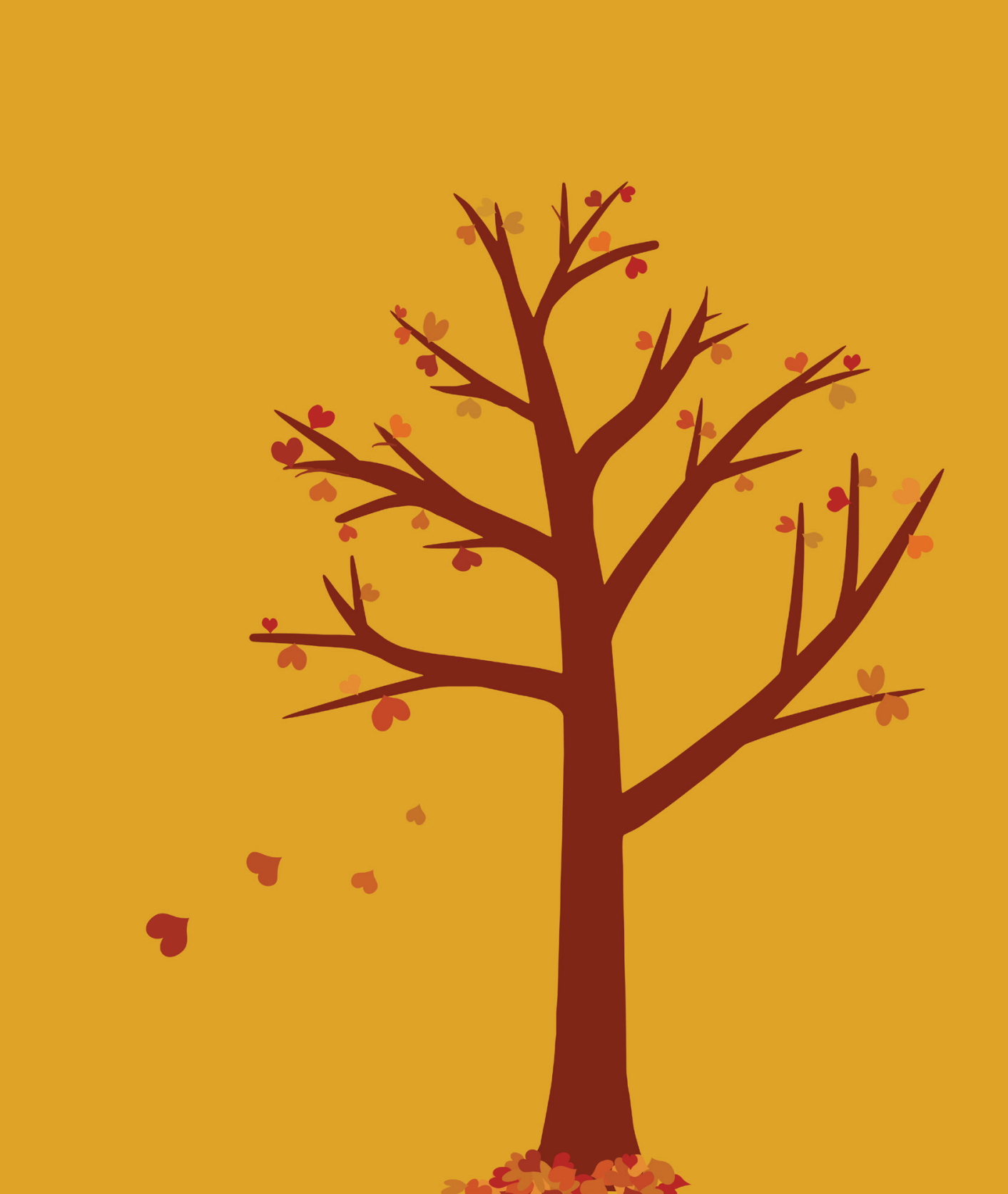


The relationship between classical vascular risk factors and cumulative recurrent cardiovascular event burden in patients with clinically manifest vascular disease: results from the UCC-SMART prospective cohort study
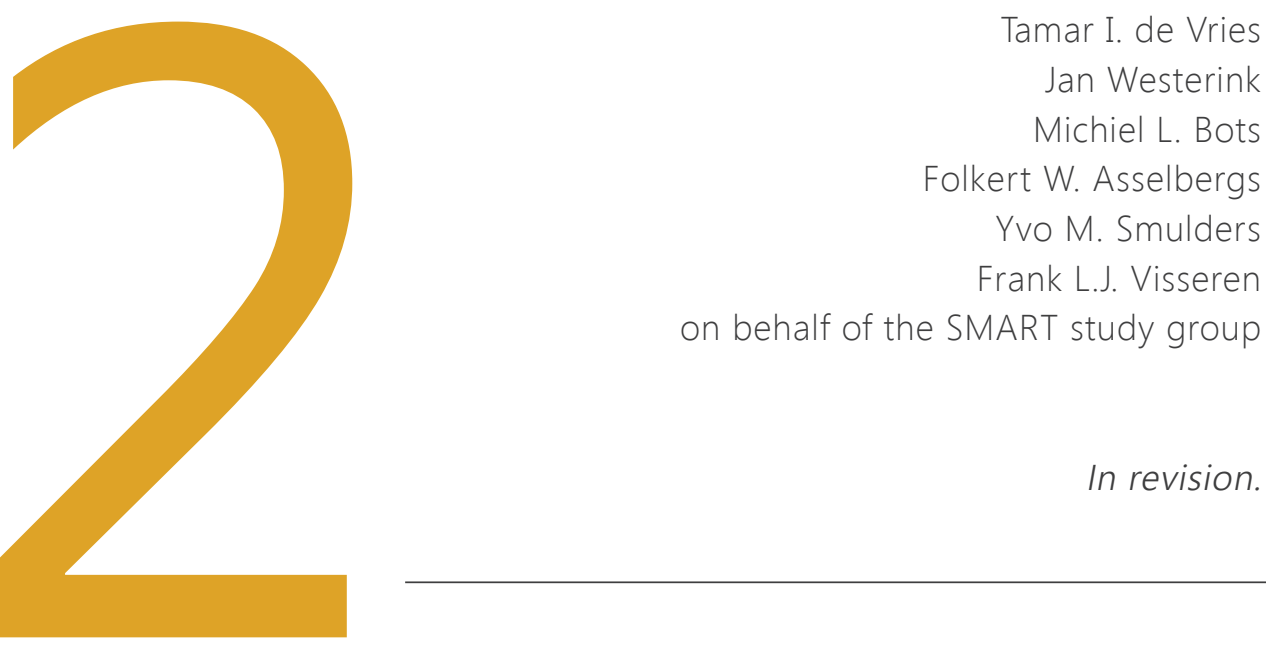

In revision. 


\section{ABSTRACT}

\section{Objective}

The aim of the current study was to assess the relationship between classical cardiovascular risk factors and the risk of not only the first recurrent atherosclerotic cardiovascular event, but also the total number of nonfatal and fatal cardiovascular events in patients with recently clinically manifest cardiovascular disease (CVD).

\section{Methods}

7239 patients with a recent first manifestation of CVD from the prospective UCC-SMART cohort study were included. Cox regression and Prentice-Williams-Peterson models were used to analyze the relationship between non-high density lipoprotein (HDL)-cholesterol, systolic blood pressure (SBP), and current smoking and total cardiovascular events, including myocardial infarction, stroke, vascular interventions, major limb events, and cardiovascular mortality.

\section{Results}

During a median follow-up of 8.9 years, 1412 patients had 1 recurrent cardiovascular event, while 1290 patients had 2 or more recurrent events, with a total of 5457 cardiovascular events during follow-up. The hazard ratios for respectively the first recurrent event and cumulative event burden using Prentice-Williams-Peterson models were 1.36 (95\% confidence interval 1.25 - 1.48) and 1.26 (1.17 - 1.35) for smoking, $1.14(1.11$ - 1.18) and 1.09 (1.06 - 1.12) for non-HDL-cholesterol, and 1.05 (1.03 - 1.07) and 1.04 (1.03 - 1.06) for SBP per $10 \mathrm{mmHg}$.

\section{Conclusions}

In a cohort of patients with established cardiovascular disease, SBP, non-HDL cholesterol, and current smoking are important risk factors for not only the first, but also subsequent recurrent events during follow up. Recurrent event analysis captures the full cumulative burden of CVD in patients. 


\section{Introduction}

In cardiovascular (CV) clinical trials investigating treatment effects and in cohort studies, there is a focus on the first CV event during follow up. In clinical practice not only the first (recurrent) CV event is relevant for health care professionals and patients, but all CV events during the next years or even during a patients' lifespan.

Evaluating only the first (recurrent) CV event during follow-up does not utilize the complete clinical information available, as subjects with a first nonfatal event can continue to be followed during a study period, and can experience numerous additional events during the course of follow-up. Ignoring these additional events may therefore not reflect the total cardiovascular disease (CVD) burden of an individual patient over time. ${ }^{1}$ Moreover, it is likely that the relation between a risk factor and an endpoint may be different for the first event compared to the total disease burden. ${ }^{2}$ This may be especially true for modifiable risk factors which may diminish in importance after the initiation of secondary prevention. Consequently, time to first event analyses may not capture the full clinical impact of a risk factor. Clinically, therefore, it is also of interest to study the effect of risk factors or treatment on the cumulative event burden. The commonly used composite endpoint including only 'hard' endpoints such as myocardial infarction, stroke, and CV death, may also not capture the clinical burden for a patient, as revascularization procedures and peripheral artery disease are also relevant events or outcomes in clinical practice in terms of loss of quality of life and economic costs. ${ }^{3}$ Investigating the relationship between risk factors and the total event burden may therefore provide additional information compared to first events in a composite endpoint alone. Several randomized clinical trials studying risk factor reduction in CV research have published results of total event analyses in the last decade, which show that recurrent event analysis are a better measure of the total disease burden. ${ }^{3-8}$ However, few total (or cumulative) event analyses have previously been published that study the relation between classical vascular risk factors and CV outcomes in an observational cohort study. 9,10

Patients with established CVD are known to be at higher risk of future (recurrent) events compared to patients without CVD. ${ }^{11}$ The study aim of the current study is to assess the relationship between classical modifiable CV risk factors blood pressure (BP), cholesterol, and smoking, and the risk of not only the first recurrent atherosclerotic CV event, but also the total number of recurrent $C V$ events in patients with a recent first manifestation of CVD. 


\section{Methods}

\section{Study population}

Data were used from the Utrecht Cardiovascular Cohort - Second Manifestations of ARTerial disease (UCC-SMART) study, an ongoing prospective cohort study at the University Medical Center Utrecht, the Netherlands. From September 1996 onwards, patients over 18 years old referred to our institution with clinically manifest vascular disease or vascular risk factors were eligible for participation. The rationale and study design of the UCC-SMART cohort study has been published previously. ${ }^{12}$ Written informed consent was obtained from all participants, and the Medical Ethics Committee of the University Medical Center Utrecht approved the study. For the current study, data were used from 7239 participants with a recent first manifestation of CVD enrolled between 1996 and 2018.

\section{Baseline measurements}

After inclusion, study participants underwent a standardized vascular screening consisting of a health questionnaire including medical history and CV risk factors, physical examination and laboratory testing in fasting state. ${ }^{12}$ Smoking was recorded as current versus past/never smoker. Non-high density lipoprotein (HDL) cholesterol was defined as total cholesterol minus HDL cholesterol, both measured in fasting state. Systolic BP (SBP) was measured using a an automatic oscillometric device on both arms, and the mean of the highest arm was used.

\section{Follow-up}

During follow-up, information on hospitalization, outpatient clinic visits and (CV) events was obtained biannually through questionnaires. All available data were collected on reported events. All events were independently evaluated by three members of the UCCSMART cohort endpoint committee. The primary endpoint of the current study consisted of non-fatal atherosclerotic CV events, including myocardial infarction, stroke, major adverse limb events (MALE), revascularization procedures, and other vascular interventions, and death due to CV causes. MALE was defined as any amputation above the forefoot and revascularization procedures of the lower limbs. Other vascular intervention included coronary revascularization, procedures of the abdominal aorta, or revascularization of the carotid arteries, renal arteries, or iliac arteries. Death was reported by the general practitioner, treating specialist, or relatives. Duration of follow-up was defined as the period between study inclusion and death, loss to follow-up, or the preselected date of 1 March 2018. In total, 561 (6.6\%) participants were lost to follow-up during the study period. 


\section{Statistical analysis}

All analyses were conducted with $\mathrm{R}$ statistical software V.3.5.1 (www.r-project.org, packages survival, Hmisc, dplyr, MASS, ggplot2). Missing values (<1\%) were imputed using predictive mean matching (areglmpute, Hmisc, R). ${ }^{13}$

Baseline clinical characteristics were presented as frequencies with percentages for categorical variables, and means with standard deviation for continuous variables.

Nonparametric mean cumulative function curves were created for total CV events using the Nelson-Aalen estimator. ${ }^{14}$ The mean cumulative function represents the expected (i.e., mean) cumulative number of events for a patient at a given point in time after inclusion. For comparative purposes, Kaplan-Meier curves were also created for first CV event, and plotted with the mean cumulative function curves. To visualize the effect of the risk factors of interest (current smoking, non-HDL cholesterol, and SBP), plots were also created stratified for the presence of the investigated risk factors: current smoking versus no current smoking; SBP $\geq 140 \mathrm{mmHg}$ versus $<140 \mathrm{mmHg}$, and non-HDL-cholesterol $\geq 2.6$ $\mathrm{mmol} / \mathrm{l}$ versus $<2.6 \mathrm{mmol} / \mathrm{L}$, based on the European Society of Cardiology thresholds. ${ }^{15}$

As the study aim was to determine the etiological relationship between the modifiable risk factors of interest, all analyses were adjusted for potential confounders based on previous literature, which included, where appropriate: age, sex, alcohol use, type of baseline vascular disease, current smoking, SBP, and non-HDL at baseline. The analyses for non-HDL cholesterol were additionally adjusted for statin therapy, and diabetes; SBP was additionally adjusted for BP-lowering medication, and estimated glomerular filtration rate. Time-to-first event was studied using Cox proportional hazard models. The composite endpoint consisted of all previously described CVD endpoints. Linearity of the relationship between non-HDL cholesterol and SBP with the outcome was assessed with restricted cubic splines. The assumption of proportionality was visually checked by plotting Schoenfeld residuals. Prentice-Williams-Peterson (PWP) analysis, an extension of the Cox proportional hazard model to model recurrent events, was performed to assess the relationship between the risk factors of interest and the total event burden, ${ }^{16}$ as it has been suggested that this is the most robust of the recurrent event modelling methods. ${ }^{2}$ The PWP model is a conditional model, meaning that patients are only a part of the risk set for event $k$ when they have experienced event $k-1$.

\section{Sensitivity analyses}

As has been recommended, several sensitivity analyses were performed to demonstrate that an apparent causal effect does not depend on the chosen statistical method. ${ }^{17,18}$ Negative binomial regression is an attractive method for recurrent event modelling because it accommodates heterogeneity among patients. It assumes that each participant has events according to their own individual, specific event rate through a random effect term which varies according to a gamma distribution. ${ }^{19-21} \mathrm{~A}$ negative binomial regression 
model was therefore used to obtain estimates of the effect of the risk factors of interest on the outcome event rate, including all nonfatal and fatal CV events. A disadvantage of negative binomial regression is that it does not take time to event into account, which may bias the results if there is unequal follow-up. ${ }^{20}$

Additional sensitivity analyses were performed using the Andersen-Gill and Wei-LinWeissfeld (WLW) models, ${ }^{22,23}$ extensions of the Cox proportional hazard model to model recurrent events. In the WLW model, individual patients are included in the risk set for event $k$ regardless of whether they have experienced event $k-1$. Both PWP and WLW use follow-up time from inclusion, a 'total time' approach, in contrast to the 'gap time' approach of Andersen-Gill, where the times between consecutive events are modelled rather than the time from baseline. ${ }^{19}$ The Andersen-Gill method assumes that all events are independent. This assumption is clearly violated, as the risk of CV events within one individual are associated. Therefore, robust standard errors may be used with the Andersen-Gill method to account for heterogeneity. ${ }^{24}$ All recurrent event analyses were limited to the first 5 events.

To investigate the role of competing events and 'soft' endpoints on the relationship between risk factors and outcomes under the different statistical methods, several additional sensitivity analyses were performed: 1) using only non-fatal CV events; and 2) including non-CV mortality in the combined endpoint, 3) using only 'hard' endpoints usually included in a composite endpoint (non-fatal MI, non-fatal stroke, and CV mortality); 4) limited to 10 year of follow-up; 5) limited to 5 year of follow-up; 6 ) limited to patients included after 2007 to study a more contemporary patient population treated under the 2007 ESC guidelines or more recent guidelines; ${ }^{25}$ and 7) limited to non-smokers.

\section{Results}

\section{Baseline characteristics}

The baseline characteristics of the 7239 patients with recent CVD at baseline included in the study population are shown in Table 1. Compared to patients who had no recurrent CV event during follow-up, patients with one or more recurrent events generally had a less favorable risk profile at baseline (Table 1). Compared to people who experience multiple recurrent events during follow-up, those who experience no recurrent events during follow-up are slightly younger, more often women, less often current smokers, have a lower systolic blood pressure (SBP), and more often used lipid-lowering drugs at baseline. They were on average recruited later in the study (37\% after 2011 , compared to only $7 \%$ in the patients who experienced more CV events). 
Table 1. Baseline characteristics of study participants with no recurrent cardiovascular (CV) event, a single recurrent $\mathrm{CV}$ event, or multiple recurrent $\mathrm{CV}$ events

\begin{tabular}{llll}
\hline & $\begin{array}{l}\text { No recurrent CV } \\
\text { events }\end{array}$ & $\begin{array}{l}\text { Single recurrent } \\
\text { CV event }\end{array}$ & $\begin{array}{l}\text { Multiple recurrent } \\
\text { CV events }\end{array}$ \\
\cline { 2 - 4 } & $\mathrm{n}=4537$ & $\mathrm{n}=1412$ & $\mathrm{n}=1290$ \\
\hline Age (years) & $59 \pm 11$ & $61 \pm 10$ & $60 \pm 10$ \\
Male sex & $3170(70 \%)$ & $1088(77 \%)$ & $989(77 \%)$ \\
Current smoking & $1271(28 \%)$ & $473(33 \%)$ & $529(41 \%)$ \\
Systolic blood pressure (mmHg) & $137 \pm 20$ & $141 \pm 20$ & $143 \pm 22$ \\
History of coronary artery disease & $2630(58 \%)$ & $826(58 \%)$ & $736(57 \%)$ \\
History of cerebrovascular disease & $1513(33 \%)$ & $362(26 \%)$ & $276(21 \%)$ \\
History of PAD or AAA & $1995(44 \%)$ & $608(43 \%)$ & $636(49 \%)$ \\
Type 2 diabetes & $577(13 \%)$ & $246(17 \%)$ & $256(20 \%)$ \\
Lipid-lowering medication & $3219(71 \%)$ & $887(63 \%)$ & $799(62 \%)$ \\
Blood pressure lowering medication & $3332(73 \%)$ & $1058(75 \%)$ & $946(73 \%)$ \\
Antiplatelet medication & $3344(74 \%)$ & $1053(75 \%)$ & $907(70 \%)$ \\
Total cholesterol (mmol/L) & $4.7 \pm 1.2$ & $4.9 \pm 1.2$ & $5.1 \pm 1.3$ \\
HDL cholesterol (mmol/L) & $1.3 \pm 0.4$ & $1.2 \pm 0.4$ & $1.2 \pm 0.3$ \\
LDL cholesterol (mmol/L) & $2.7 \pm 1.0$ & $3.0 \pm 1.1$ & $3.2 \pm 1.1$ \\
Serum creatinine (mmol/L; median) & $85(75-97)$ & $89(78-101)$ & $89(78-103)$ \\
Inclusion year in SMART & & & \\
1996-2000 & $498(11 \%)$ & $403(29 \%)$ & $457(35 \%)$ \\
2001-2010 & $2373(52 \%)$ & $829(59 \%)$ & $743(58 \%)$ \\
2011-2018 & $1666(37 \%)$ & $180(13 \%)$ & $90(7 \%)$ \\
\hline Abbrevations: PAD & & & \\
\hline
\end{tabular}

Abbreviations: $P A D=$ peripheral artery disease; $A A A=$ abdominal aortic aneurysm; $H D L=$ high-density lipoprotein; $L D L=$ low-density lipoprotein. Presented as $n(\%)$ for categorical data, mean \pm standard deviation for continuous data, unless stated otherwise.

\section{First and total events during follow-up}

During a median follow-up of 8.9 years (interquartile range [IQR] 4.8-9.1), 1,412 patients (19.5\%) had one CV event during follow-up, and 1,290 patients (17.8\%) had multiple CV events during follow-up (Figure 1). During the course of the study, a total of 5,457 recurrent CV events occurred, of which 2,702 were first events within the study period (Figure 2). The first recurrent event took place after a median of 3.2 years (IQR 0.9-7.1) from inclusion. There were an additional 2,755 CV events that occurred after the first event during the course of the cohort study. The second recurrent event took place after a median of 1.1 years (IQR 0.2-4.1) after the first recurrent event. 
Figure 1. Number of cardiovascular events per patient during follow-up.

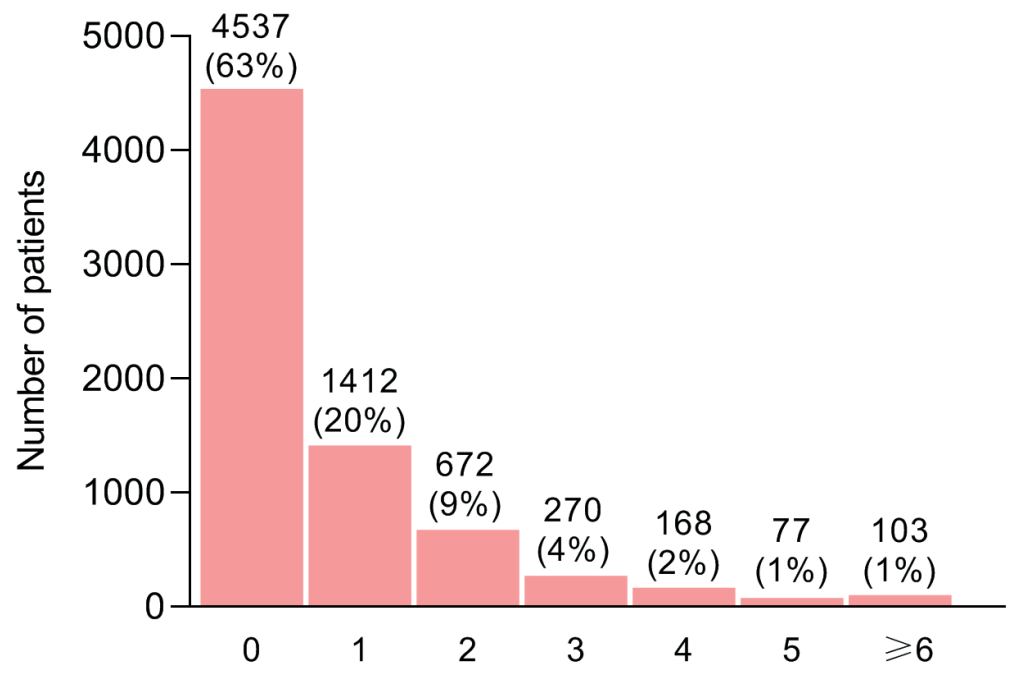

Cardiovascular events per patient during follow-up

Figure 2. Number of first and total subsequent CV total events and by individual endpoints.

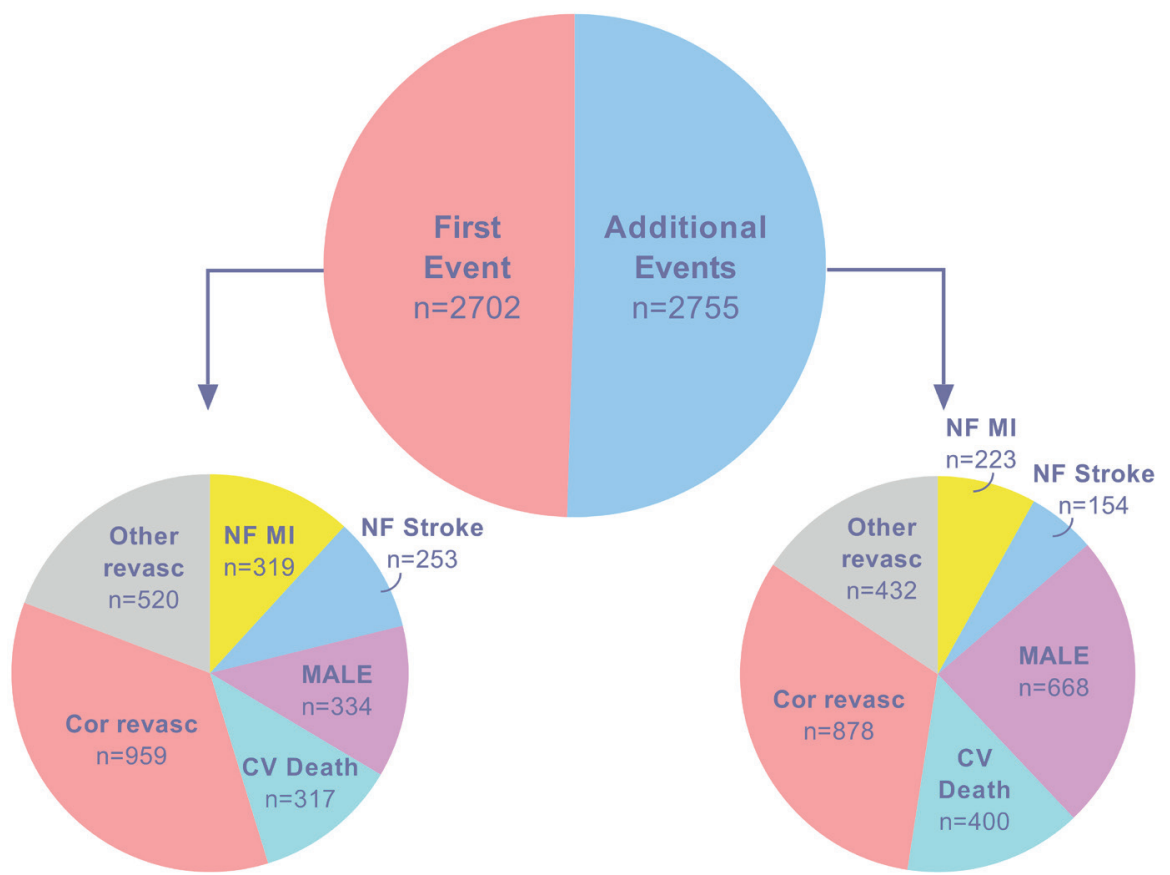

Abbreviations: $N F=$ non-fatal; $M I=$ myocardial infarction; $M A L E=$ major adverse limb events; $C V=$ cardiovascular; revasc = revascularization; cor = coronary. 
In comparison, there was a higher proportion of MALE and CV death among the additional events than among the first events (24.2\% versus $12.4 \%$ and 14.5 versus $11.7 \%$, respectively). In contrast, there were less non-fatal myocardial infarctions ( $8.1 \%$ versus $11.8 \%$ ) non-fatal strokes (5.6\% versus $9.4 \%$ ), coronary revascularization (31.9\% versus $35.5 \%)$, and other revascularizations (15.7\% versus 19.2\%). Figure 3 shows the KaplanMeier curve and mean cumulative function plot for the first and total CV events, respectively.

\section{Relation between classical risk factors and total events}

Figure 4 shows the Kaplan-Meier curve and mean cumulative function plot for the first and total new CV events, respectively, stratified for the presence of each risk factor. Table 2 shows the relationship between the classical risk factors of interest and (total) CV events as estimated by the different statistical models. Traditional timeto-first-event Cox models showed significant relationships with first recurrent CV events for current smoking (HR 1.36, 95\% confidence interval [CI] 1.25-1.48), non-HDLcholesterol (HR 1.14 per mmol/L; 95\%Cl 1.11-1.18), and SBP (HR 1.05 per $10 \mathrm{mmHg}$; 95\% $\mathrm{Cl}$ 1.03-1.07). The PWP models showed significant relationship between the cumulative recurrent event burden and current smoking (HR 1.26; 95\% Cl $1.17-1.35)$, non-HDL cholesterol (HR 1.09; 95\%Cl 1.06 - 1.12), and SBP (HR 1.04; 95\% Cl 1.03 - 1.06).

Figure 3. Mean cumulative functions and Kaplan-Meier curves for cardiovascular (CV) events.

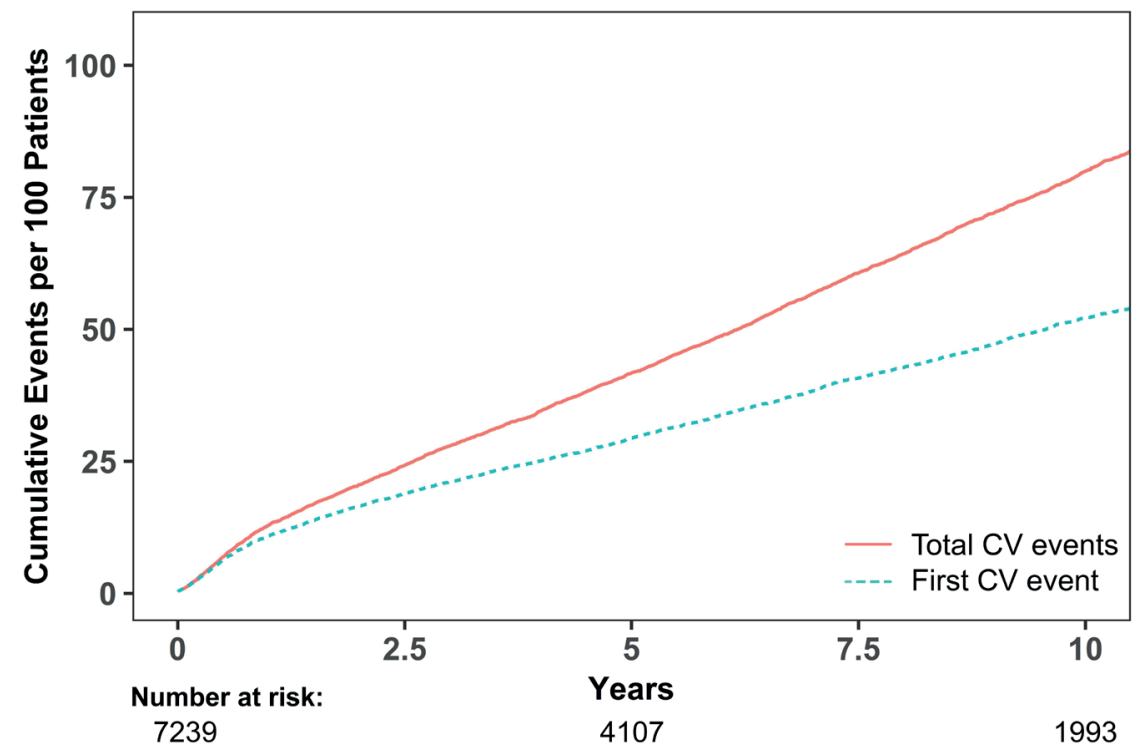

The mean cumulative function curve depicts the expected total number of recurrent cardiovascular (CV) events for a given 100 patients in the study population at a given time after randomization. In contrast, the Kaplan-Meier curve depicts the expected number of patients with a first recurrent CV event at any given time per 100 patients. 
Figure 4. Mean cumulative functions (for recurrent events) and Kaplan-Meier curves (for first events) for the risk of cardiovascular (CV) events, stratified for (A) smoking status, (B) non-high density lipoprotein (HDL) cholesterol, and (C) systolic blood pressure (SBP)

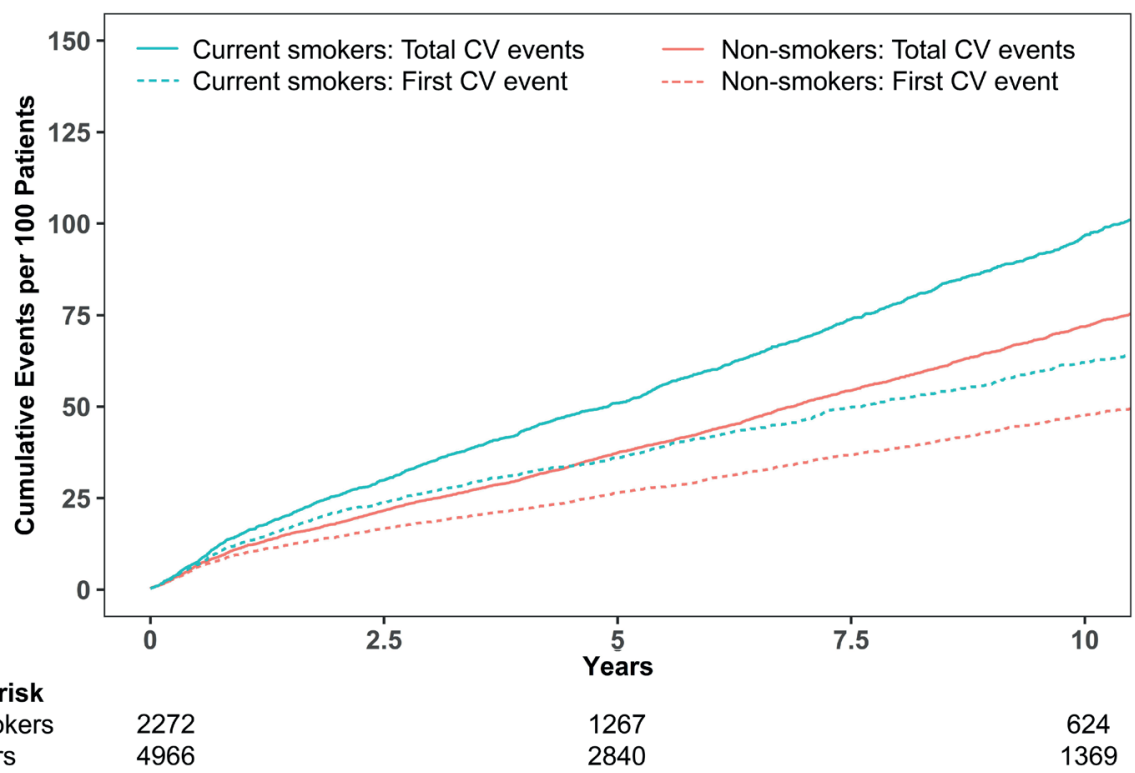

(B)

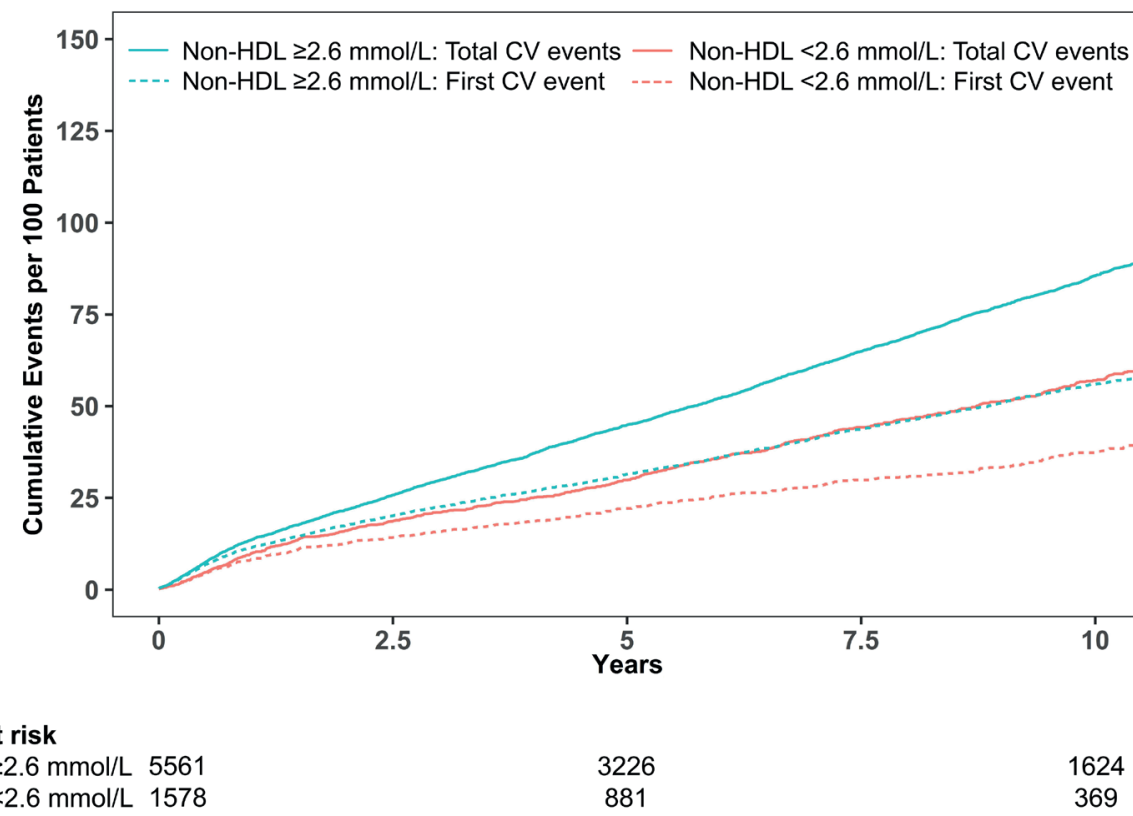

Number at risk

Non-HDL $\geq 2.6 \mathrm{mmol} / \mathrm{L} 5561$

881

369

The mean cumulative function curve depicts the expected total number of recurrent cardiovascular (CV) events for a given 100 patients in the study population at a given time after randomization. In contrast, the Kaplan-Meier curve depicts the expected number of patients with a first recurrent CV event at any given time per 100 patients. 
Figure 4. (continued)

(C)

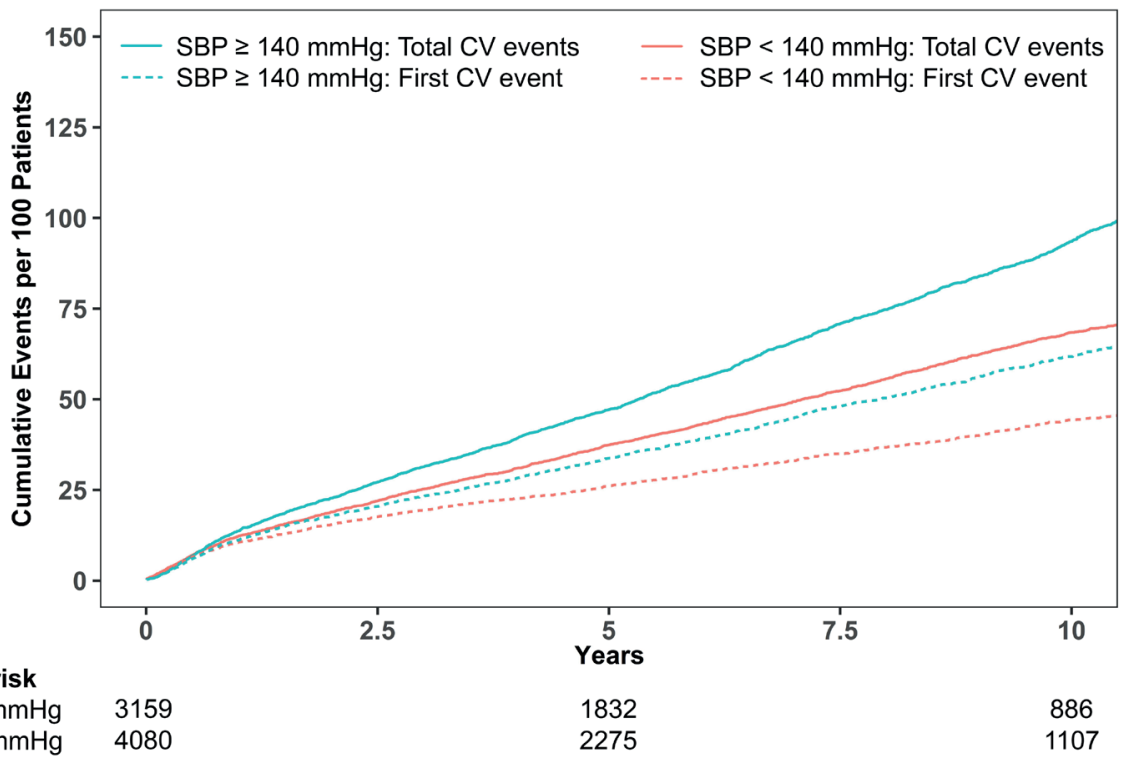

\section{Sensitivity analyses}

Negative binomial regression and Andersen-Gill showed consistent results with regards to direction of the relation, but not in magnitude (Table 2). For all risk factors, negative binomial regression showed stronger relationships than both Andersen-Gill and PWP. For all three risk factors, the relationship between event and risk factor was stronger for the fifth than for the first recurrent event, as shown by the WLW analysis. The results of the other sensitivity analyses are shown in Table 3. There were no important differences in the sensitivity analyses compared to the main analyses, with overlapping confidence intervals.

\section{Discussion}

In the current paper, we demonstrated that in patients with recently clinically manifest vascular disease, the modifiable risk factors current smoking, non-HDL cholesterol, and SBP, are not only related to the risk of a first recurrent CV event during follow-up, but also with total recurrent $\mathrm{CV}$ events over long-term follow-up.

Although not necessarily particularly surprising, the results of the present study confirm that better treatment of classical risk factors is necessary in patients with clinically manifest vascular disease to prevent not just the first recurrent vascular event, but even more so the total burden of events. This is not only true for the hard (classical MACE) endpoints, 
Table 2. Relation between traditional risk factors and first or total subsequent recurrent CV events according to different statistical methods

\begin{tabular}{|c|c|c|c|c|}
\hline & & Current smoking & $\begin{array}{l}\text { Non-HDL-c } \\
\text { (per mmol/L) }\end{array}$ & $\begin{array}{l}\text { SBP } \\
(\text { per 10mmHg) }\end{array}$ \\
\hline & Events $^{\star}$ & $\mathrm{HR}(95 \% \mathrm{Cl})$ & $\mathrm{HR}(95 \% \mathrm{Cl})$ & $\mathrm{HR}(95 \% \mathrm{Cl})$ \\
\hline Prentice Williams Peterson & 5138 & $1.26(1.17-1.35)$ & $1.09(1.06-1.12)$ & $1.04(1.03-1.06)$ \\
\hline Negative binomial regression & 5138 & $1.33(1.22-1.45)$ & $1.23(1.19-1.28)$ & $1.07(1.05-1.10)$ \\
\hline Andersen-Gill & 5138 & $1.35(1.24-1.47)$ & $1.13(1.09-1.16)$ & $1.05(1.04-1.07)$ \\
\hline Wei-Lin-Weissfeld & 2702 & $1.41(1.28-1.56)$ & $1.15(1.10-1.21)$ & $1.06(1.04-1.09)$ \\
\hline \multicolumn{5}{|l|}{$\underline{\text { Stratified per event }}$} \\
\hline \multicolumn{5}{|l|}{ Prentice Williams Peterson } \\
\hline 1st event & 2702 & $1.36(1.25-1.48)$ & $1.14(1.10-1.19)$ & $1.05(1.03-1.07)$ \\
\hline 2nd event & 1290 & $1.17(1.02-1.35)$ & $1.09(1.03-1.16)$ & $1.03(1.00-1.07)$ \\
\hline 3rd event & 618 & $1.04(0.84-1.29)$ & $0.99(0.93-1.06)$ & $1.09(1.04-1.14)$ \\
\hline 4th event & 348 & $1.03(0.77-1.38)$ & $1.02(0.95-1.08)$ & $1.00(0.94-1.06)$ \\
\hline 5th event & 180 & $1.40(0.90-2.17)$ & $1.03(0.93-1.13)$ & $1.03(0.95-1.12)$ \\
\hline \multicolumn{5}{|l|}{ Wei-Lin-Weissfeld } \\
\hline 1st event & 2702 & $1.36(1.25-1.48)$ & $1.14(1.10-1.19)$ & $1.05(1.03-1.07)$ \\
\hline 2nd event & 1290 & $1.49(1.32-1.68)$ & $1.18(1.12-1.25)$ & $1.06(1.03-1.09)$ \\
\hline 3rd event & 618 & $1.44(1.21-1.72)$ & $1.13(1.05-1.22)$ & $1.10(1.06-1.14)$ \\
\hline 4th event & 348 & $1.43(1.13-1.81)$ & $1.13(1.04-1.24)$ & $1.12(1.06-1.18)$ \\
\hline 5th event & 180 & $1.56(1.12-2.17)$ & $1.20(1.10-1.31)$ & $1.10(1.02-1.18)$ \\
\hline
\end{tabular}

$\overline{H R}=$ hazard ratio; $\mathrm{Cl}=$ confidence interval; $\mathrm{HDL}=$ high-density lipoprotein; $S B P=$ systolic blood pressure * Limited to the first 5 recurrent events

but for all (non-fatal and fatal) endpoints. These results may be of use in communicating the benefit of risk factor treatment with patients in clinical practice. Although the effect sizes are smaller for the total recurrent events compared to only the first recurrent event, they are still clinically very important. Smoking at baseline is associated with a $26 \%$ increase in hazard for any recurrent event. As we have only used baseline smoking status in the current analysis, this does not account for people who quit smoking after baseline. Likely, the effect size of those patients who keep smoking throughout the study followup is larger. This is corroborated by previous analyses showing that smoking cessation importantly decreases the risk of future events. But also the hazard ratios for non-HDL-C and SBP are clinically important; a difference of $3 \mathrm{mmol} / \mathrm{L}$ in baseline non-HDL-C is associated with a $30 \%$ increase in the hazard for any recurrent event; a difference of 40 $\mathrm{mmHg}$ in baseline SBP is associated with a $17 \%$ increase in hazards. 
Table 3. Results of the sensitivity analyses: (A) the results from the main analysis of the study, (B) studying only non-fatal events, (C) studying all non-fatal events and all-cause mortality, (D) only major cardiovascular events (non-fatal myocardial infarction, non-fatal stroke, and cardiovascular mortality), (E) limiting the follow-up to 10 years from baseline, (F) limiting the follow-up to 5 years from baseline, (G) limiting the analysis to only patients included after 2007; and $(\mathrm{H})$ including the analysis to non-smokers only.

\begin{tabular}{|c|c|c|c|c|c|}
\hline & & & $\begin{array}{l}\text { Current } \\
\text { smoking }\end{array}$ & $\begin{array}{l}\text { Non-HDL-c } \\
\text { (per mmol/L) }\end{array}$ & $\begin{array}{l}\text { SBP } \\
\text { (per 10mmg) }\end{array}$ \\
\hline Scenario & Model & Events* & $\mathrm{HR}(95 \% \mathrm{Cl})$ & HR $(95 \% \mathrm{Cl})$ & $\mathrm{HR}(95 \% \mathrm{Cl})$ \\
\hline \multirow[t]{2}{*}{ Main analysis } & First event' ${ }^{+}$ & 2702 & $1.36(1.25-1.48)$ & $1.14(1.11-1.18)$ & $1.05(1.03-1.07)$ \\
\hline & PWP & 5138 & $1.26(1.17-1.35)$ & $1.09(1.06-1.12)$ & $1.04(1.03-1.06)$ \\
\hline \multirow{2}{*}{$\begin{array}{l}\text { Only non-fatal } \\
\text { events }\end{array}$} & First event & 2385 & $1.34(1.22-1.46)$ & $1.15(1.10-1.19)$ & $1.05(1.03-1.07)$ \\
\hline & PWP & 4457 & $1.20(1.12-1.29)$ & $1.09(1.06-1.12)$ & $1.05(1.03-1.06)$ \\
\hline \multirow{2}{*}{$\begin{array}{l}\text { Non-fatal events and } \\
\text { all-cause mortality }\end{array}$} & First event & 3211 & $1.45(1.34-1.57)$ & $1.12(1.08-1.15)$ & $1.04(1.03-1.06)$ \\
\hline & PWP & 5940 & $1.33(1.25-1.41)$ & $1.08(1.05-1.11)$ & $1.04(1.03-1.06)$ \\
\hline \multirow{2}{*}{$\begin{array}{l}\text { Major cardiovascular } \\
\text { events }\end{array}$} & First event & 1288 & $1.64(1.45-1.85)$ & $1.08(1.03-1.14)$ & $1.03(1.01-1.06)$ \\
\hline & PWP & 1660 & $1.52(1.35-1.72)$ & $1.07(1.02-1.12)$ & $1.04(1.01-1.06)$ \\
\hline \multirow{2}{*}{$\begin{array}{l}\text { Limited to } 10 \text { years } \\
\text { follow-up }\end{array}$} & First event & 2368 & $1.39(1.27-1.52)$ & $1.14(1.10-1.19)$ & $1.05(1.03-1.07)$ \\
\hline & PWP & 4142 & $1.28(1.19-1.38)$ & $1.09(1.06-1.13)$ & $1.05(1.03-1.06)$ \\
\hline \multirow{2}{*}{$\begin{array}{l}\text { Limited to } 5 \text { years } \\
\text { follow-up }\end{array}$} & First event & 1691 & $1.40(1.25-1.55)$ & $1.13(1.08-1.18)$ & $1.04(1.02-1.07)$ \\
\hline & PWP & 2623 & $1.28(1.17-1.40)$ & $1.09(1.06-1.13)$ & $1.04(1.02-1.06)$ \\
\hline \multirow{2}{*}{$\begin{array}{l}\text { Limited to patients } \\
\text { included after } 2007\end{array}$} & First event & 644 & $1.40(1.17-1.67)$ & $1.12(1.04-1.22)$ & $1.04(1.00-1.08)$ \\
\hline & PWP & 1069 & $1.32(1.15-1.53)$ & $1.09(1.02-1.18)$ & $1.03(0.99-1.06)$ \\
\hline \multirow[t]{2}{*}{ Only never-smokers } & First event & 485 & $\mathrm{~N} / \mathrm{A}$ & $1.16(1.06-1.27)$ & $1.04(1.00-1.09)$ \\
\hline & PWP & 841 & $\mathrm{~N} / \mathrm{A}$ & $1.14(1.06-1.23)$ & $1.02(0.99-1.06)$ \\
\hline
\end{tabular}

Abbreviations: $H R=$ hazard ratio; $95 \% \mathrm{Cl}=95 \%$ confidence interval; $H D L=$ high-density lipoprotein; $S B P=$ systolic blood pressure; PWP = Prentice, Williams, and Peterson; N/A = not applicable

* Limited to the first 5 recurrent events

+ Cox regression for time to first events

Multiple events over time in a subject are associated with a large burden for the patient, decreased quality of life, and higher costs. This is important not only on a patient level, but also for the health care systems as multiple events in a single patient lead to the use of more resources, due to hospitalizations, tests, additional (surgical) interventions, and physician visits. 
In recent years, several randomized controlled trials published the effect of trial interventions on the total CV event burden. In the ODYSSEY OUTCOMES trial, which studied the addition of the PCSK9 monoclonal antibody alirocumab to intensive statin therapy after acute coronary syndrome (ACS), nonfatal CV events were prevented in 162 patients during follow-up, while a total of 327 nonfatal CV events were prevented with alirocumab compared to placebo. ${ }^{7}$ Similarly, in the REDUCE-IT trial, icosapent ethyl prevented 196 first events compared to placebo, but 470 total events (from a composite of CV death, nonfatal myocardial infarction, nonfatal stroke, coronary revascularization, or hospitalization for unstable angina) in patients with either established CVD or diabetes mellitus with additional risk factors. ${ }^{8}$ In the IMPROVE-IT trial, the addition of ezetimibe to simvastatin prevented 170 first events compared to placebo, and 421 total events. ${ }^{5}$ In these trials, the efficacy of these therapies is thus amplified by studying the total event burden, giving a better reflection of the clinical impact in the respective patient populations.

In the current study, the total CV event burden is double the burden of first CV events. Interestingly, the sensitivity analysis using the Wei-Lin-Weissfeld method shows consistently for all three risk factors that the association between risk factor and fifth event of a patient is larger than the association between risk factor and first event of a patient, suggesting that these classical risk factors become more important for subsequent events. This is interesting, as lipid lowering and BP lowering are cornerstones of secondary prevention in international guidelines. One would therefore expect that the relationship between lipid or BP levels on baseline and later recurrent events should be diminished. One hypothesis is that the group who has multiple recurrent events is most resistant to therapy. If part of the population meets the treatment targets, the effect of the risk factors in those who do not meet the treatment targets may be relatively more important. However, there may also be a statistical carry-over effect from the risk factor from event $k$ to event $k+1$, and it has been suggested that this statistical methodology may lead to overestimation of the effect.19 Finally, it should be realized that the WLW model was a sensitivity analysis. Nonetheless, it is an interesting finding that warrants attention, as it indicates that there are patients with important residual risk from modifiable risk factors, perhaps even suggesting that the preventive treatment may be inadequate in some patients under treatment in this tertiary center.

The PWP strata-specific results give an opposite result from the WLW strata-specific results. Looking at the strata-specific coefficients using the PWP approach, however, it seems as though the association with the risk factors decreases between cumulative events. However, methodologically this is to be expected, as only patients who have had a $k-1$, and who are still at risk (i.e. who haven't died) are in the risk set for event $k$, and the risk of event k-1 was also associated with the risk factors of interest. Thus, this approach 
give the impression that these risk factors become less important after more recurrent events, while this may very well be inherent to the statistical methodology.

As there are still many unanswered questions about total event analysis, we would not recommend replacing time-to-first event analyses with recurrent event analysis. However, due to the clinical impact of the total event burden compared to first events alone, we would recommend that recurrent event analysis should be used more often in observational research to study the impact of risk factors on the total disease burden.

The current study has several strengths. Firstly, the total event burden is a metric that better captures the entire clinical impact of risk factors than first events only. Additionally, this gives an increased statistical power to study the effects of risk factors on outcomes. ${ }^{26}$ As the UCC-SMART cohort has a long follow-up with a large number of $\mathrm{CV}$ events, this study is very suitable for studying these questions. Additional strengths include the prospective study design, and the extensive and systematic availability of data on potential confounders. Finally, we performed several sensitivity analyses to confirm the validity of the results, including limiting the follow-up time to 10 and 5 years, as there may be up to 20 years follow-up between baseline measurements and end of follow-up for some patients in the UCC-SMART cohort, which may potentially dilute the effect on recurrent events, especially since risk factor management was less stringent in the earlier years of the UCC-SMART cohort.

Several potential limitations of this study also need to be acknowledged. Firstly, as mentioned before, only baseline measurements are studied. The current study therefore differs from the previously mentioned randomized controlled trials, where an intervention strategy is studied that influences the risk factor of interest (i.e. LDL-cholesterol). Additionally, all endpoints are weighed equally in the endpoint, while in clinical practice, not all endpoints have equal clinical relevance. A weighted effect measure, which has already been proposed in previous literature, could solve this issue. ${ }^{27}$ Such methods should be examined in future studies. Furthermore, the statistical methods for analyzing subsequent events make certain assumptions regarding the relationship between subsequent events which may not be met. Therefore, to ascertain the validity of the results, we used several different statistical methodologies. Although the different statistical methods do not show consistency in magnitude of the relationship, there is consistency of findings in terms of direction. It has previously been demonstrated that the choice of statistical method for recurrent event analysis can impact the results and conclusion. ${ }^{2,9,28}$ It is thus unclear which statistical method should be preferred for recurrent event analysis in observational CVD studies, although it has been suggested that PWP are models may be the most robust option. ${ }^{2}$ 
Simulation studies have suggested that PWP should be the recommended approach for analysis of recurrent events in trials,29 although it is unclear whether this is also true for cohort studies. We therefore chose to use PWP as primary analyses and the other statistical methods as sensitivity analyses. Furthermore, the PWP models do not violate the assumption of proportionality. Finally, observational recurrent event analysis is at risk of index event bias, which generally tends to bias towards the null. ${ }^{30}$ This means that the hazard ratios found in the current study may be underestimations of the true effect sizes.

In conclusion, in a cohort of patients with a recent first manifestation of established $C V D$, the total $C V$ event burden during follow up was twice as high as the number of first events. SBP, non-HDL cholesterol, and current smoking are important modifiable risk factors for not only the first, but also for subsequent CV events, which are of great importance for patient in clinical practice. These results confirm the importance of classical risk factors for total CV burden, underlining the necessity of optimal treatment of CV risk factors. 


\section{References}

1. Anker SD, McMurray JJ V. Time to move on from "time-to-first": should all events be included in the analysis of clinical trials? Eur Heart J. 2012;33:2764-2765.

2. Yang W, Jepson C, Xie D, Roy JA, Shou H, Hsu JY, Anderson AH, Landis JR, He J, Feldman HI. Statistical Methods for Recurrent Event Analysis in Cohort Studies of CKD. Clin J Am Soc Nephrol. 2017;12:2066-2073.

3. Tikkanen MJ, Szarek M, Fayyad R, Holme I, Cater NB, Faergeman O, Kastelein JJP, Olsson AG, Larsen ML, Lindahl C, Pedersen TR. Total cardiovascular disease burden: comparing intensive with moderate statin therapy insights from the IDEAL (Incremental Decrease in End Points Through Aggressive Lipid Lowering) trial. J Am Coll Cardiol. 2009;54:2353-2357.

4. Murphy SA, Cannon CP, Wiviott SD, McCabe CH, Braunwald E. Reduction in recurrent cardiovascular events with intensive lipid-lowering statin therapy compared with moderate lipid-lowering statin therapy after acute coronary syndromes from the PROVE IT-TIMI 22 (Pravastatin or Atorvastatin Evaluation and Infection T. J Am Coll Cardiol. 2009;54:2358-2362.

5. Murphy SA, Cannon CP, Blazing MA, Giugliano RP, White JA, Lokhnygina Y, Reist C, Im K, Bohula EA, Isaza D, Lopez-Sendon J, Dellborg M, Kher U, Tershakovec AM, Braunwald E. Reduction in Total Cardiovascular Events With Ezetimibe/Simvastatin Post-Acute Coronary Syndrome: The IMPROVE-IT Trial. J Am Coll Cardiol. 2016;67:353-361.

6. LaRosa JC, Deedwania PC, Shepherd J, Wenger NK, Greten H, DeMicco DA, Breazna A. Comparison of 80 versus $10 \mathrm{mg}$ of atorvastatin on occurrence of cardiovascular events after the first event (from the Treating to New Targets [TNT] trial). Am J Cardiol. 2010;105:283-287.

7. Szarek M, White HD, Schwartz GG, Alings M, Bhatt DL, Bittner VA, Chiang C-E, Diaz R, Edelberg JM, Goodman SG, Hanotin C, Harrington RA, Jukema JW, Kimura T, Kiss RG, Lecorps G, Mahaffey KW, Moryusef A, Pordy R, Roe MT, Tricoci P, Xavier D, Zeiher AM, Steg PG. Alirocumab Reduces Total Nonfatal Cardiovascular and Fatal Events: The ODYSSEY OUTCOMES Trial. J Am Coll Cardiol. 2019;73:387-396.

8. Bhatt DL, Steg PG, Miller M, Brinton EA, Jacobson TA, Ketchum SB, Doyle RTJ, Juliano RA, Jiao L, Granowitz C, Tardif J-C, Gregson J, Pocock SJ, Ballantyne CM. Effects of Icosapent Ethyl on Total Ischemic Events: From REDUCE-IT. J Am Coll Cardiol. 2019;73:2791-2802.

9. Ip EH, Efendi A, Molenberghs G, Bertoni AG. Comparison of risks of cardiovascular events in the elderly using standard survival analysis and multiple-events and recurrent-events methods. BMC Med Res Methodol. 2015;15:15.

10. Chinwong S, Patumanond J, Chinwong D, Hall JJ, Phrommintikul A. Reduction in total recurrent cardiovascular events in acute coronary syndrome patients with low-density lipoprotein cholesterol goal $<70 \mathrm{mg} / \mathrm{dL}$ : a real-life cohort in a developing country. Ther Clin Risk Manag. 2016;12:353-360.

11. Engelen SE, van der Graaf Y, Stam-Slob MC, Grobbee DE, Cramer MJ, Kappelle LJ, de Borst GJ, Visseren FLJ, Westerink J. Incidence of cardiovascular events and vascular interventions in 
patients with type 2 diabetes. Int J Cardiol. 2017;248:301-307.

12. Simons PC, Algra A, van de Laak MF, Grobbee DE, van der Graaf Y. Second manifestations of ARTerial disease (SMART) study: rationale and design. Eur J Endocrinol. 1999;15:773-781.

13. Donders AR, van der Heijden GJ, Stijnen T, Moons KG. Review: A gentle introduction to imputation of missing values. J Clin Epidemiol. 2006;59:1087-1091.

14. Aalen O. Nonparametric Inference for a Family of Counting Processes. Ann Stat. 1978;6:701-726.

15. Piepoli MF, Hoes AW, Agewall S, Albus C, Brotons C, Catapano AL, Cooney M-T, Corra U, Cosyns B, Deaton C, Graham I, Hall MS, Hobbs FDR, Lochen M-L, Lollgen H, Marques-Vidal P, Perk J, Prescott E, Redon J, Richter DJ, Sattar N, Smulders Y, Tiberi M, van der Worp HB, van Dis I, Verschuren WMM, Authors/Task Force Members. 2016 European guidelines on cardiovascular disease prevention in clinical practice: the Sixth Joint Task Force of the European Society of Cardiology and Other Societies on Cardiovascular Disease Prevention in Clinical Practice. Eur Heart J. 2016;37:2315-2381.

16. Prentice RL, Williams BJ, Peterson AV. On the regression analysis of multivariate failure time data. Biometrika. 1981;68:373-379.

17. Rauch G, Kieser M, Binder H, Bayes-Genis A, Jahn-Eimermacher A. Time-to-first-event versus recurrent-event analysis: points to consider for selecting a meaningful analysis strategy in clinical trials with composite endpoints. Clin Res Cardiol. 2018;107:437-443.

18. Rogers JK, Pocock SJ, McMurray J V, Granger CB, Michelson EL, Ostergren J, Pfeffer MA, Solomon SD, Swedberg K, Yusuf S. Analysing recurrent hospitalizations in heart failure: a review of statistical methodology, with application to CHARM-Preserved. Eur J Heart Fail. 2014;16:33-40.

19. Rogers JK, Yaroshinsky A, Pocock SJ, Stokar D, Pogoda J. Analysis of recurrent events with an associated informative dropout time: Application of the joint frailty model. Stat Med. 2016;35:2195-2205.

20. Glynn RJ, Buring JE. Ways of measuring rates of recurrent events. BMJ. 1996;312:364-367.

21. Jahn-Eimermacher A. Comparison of the Andersen-Gill model with poisson and negative binomial regression on recurrent event data. Comput Stat Data Anal. 2008;52:4989-4997.

22. Andersen PK, Gill RD. Cox's Regression Model for Counting Processes: A Large Sample Study. Ann Stat. 1982;10:1100-1120.

23. Wei LJ, Lin DY, Weissfeld L. Regression Analysis of Multivariate Incomplete Failure Time Data by Modeling Marginal Distributions. J Am Stat Assoc. 1989;84:1065-1073.

24. Lin DY, Wei LJ. The Robust Inference for the Cox Proportional Hazards Model. J Am Stat Assoc. 1989;84:1074-1078.

25. Graham I, Atar D, Borch-Johnsen K, Boysen G, Burell G, Cifkova R, Dallongeville J, De Backer G, Ebrahim S, Gjelsvik B, Herrmann-Lingen C, Hoes A, Humphries S, Knapton M, Perk J, Priori SG, Pyorala K, Reiner Z, Ruilope L, Sans-Menendez S, Scholte op Reimer W, Weissberg P, Wood D, Yarnell J, Zamorano JL, Walma E, Fitzgerald T, Cooney MT, Dudina A. European guidelines on cardiovascular disease prevention in clinical practice: executive summary: Fourth Joint Task Force of the European Society of Cardiology and Other Societies on Cardiovascular Disease 
Prevention in Clinical Practice (Constituted by r. Eur Heart J. 2007;28:2375-2414.

26. Rogers JK, Jhund PS, Perez A-C, Bohm M, Cleland JG, Gullestad L, Kjekshus J, van Veldhuisen DJ, Wikstrand J, Wedel H, McMurray JJ V, Pocock SJ. Effect of rosuvastatin on repeat heart failure hospitalizations: the CORONA Trial (Controlled Rosuvastatin Multinational Trial in Heart Failure). JACC Heart Fail. 2014;2:289-297.

27. Rauch G, Kunzmann K, Kieser M, Wegscheider K, König J, Eulenburg C. A weighted combined effect measure for the analysis of a composite time-to-first-event endpoint with components of different clinical relevance. Stat Med. 2018;37:749-767.

28. Bakal JA, Roe MT, Ohman EM, Goodman SG, Fox KAA, Zheng Y, Westerhout CM, Hochman JS, Lokhnygina $Y$, Brown EB, Armstrong PW. Applying novel methods to assess clinical outcomes: insights from the TRILOGY ACS trial. Eur Heart J. 2015;36:385-92a.

29. Ozga A-K, Kieser M, Rauch G. A systematic comparison of recurrent event models for application to composite endpoints. BMC Med Res Methodol. 2018;18:2.

30. Dahabreh IJ, Kent DM. Index event bias as an explanation for the paradoxes of recurrence risk research. JAMA. 2011;305:822-823. 


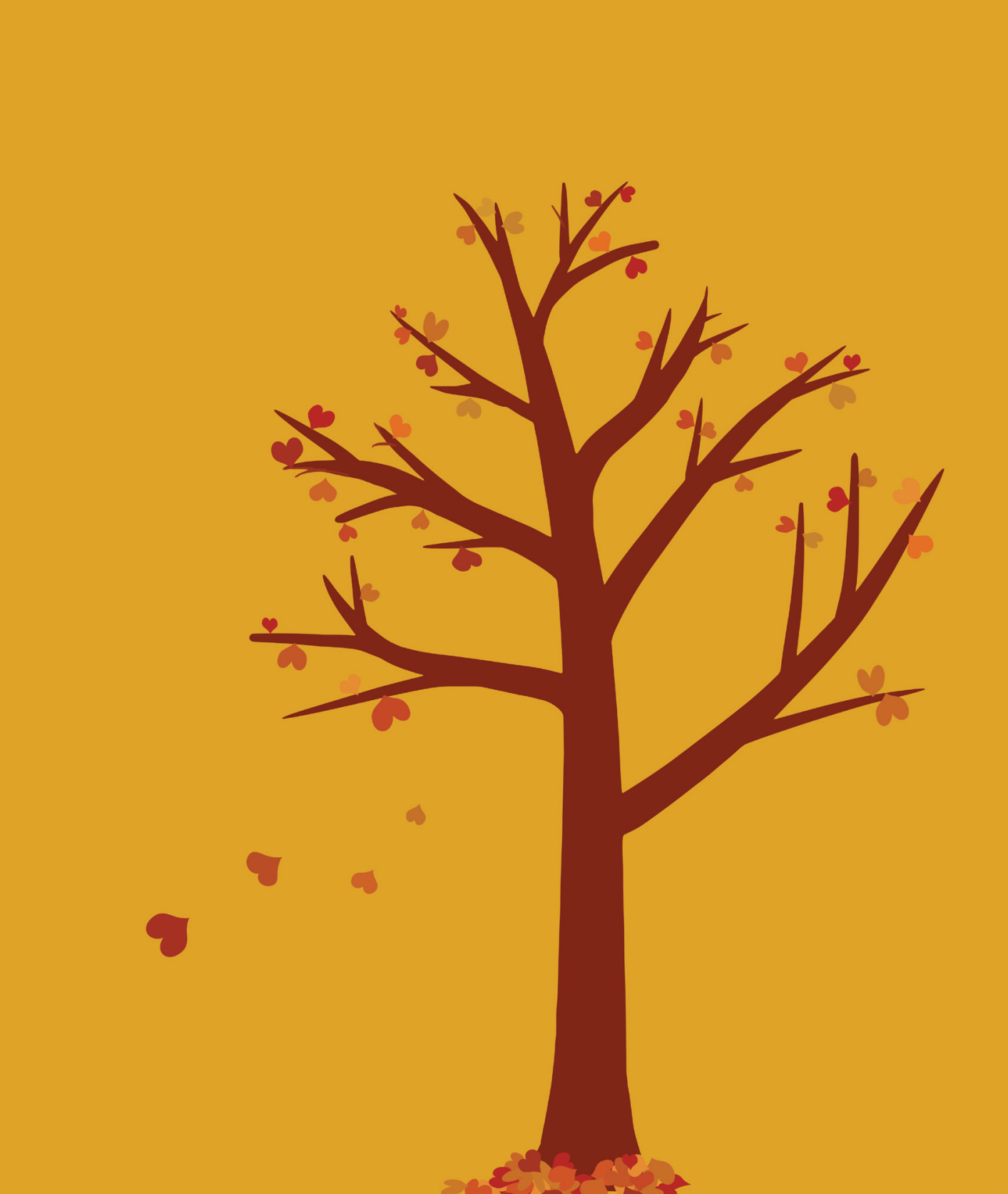




\title{
Thyroid-stimulating hormone levels in the normal range and incident type 2 diabetes mellitus
}

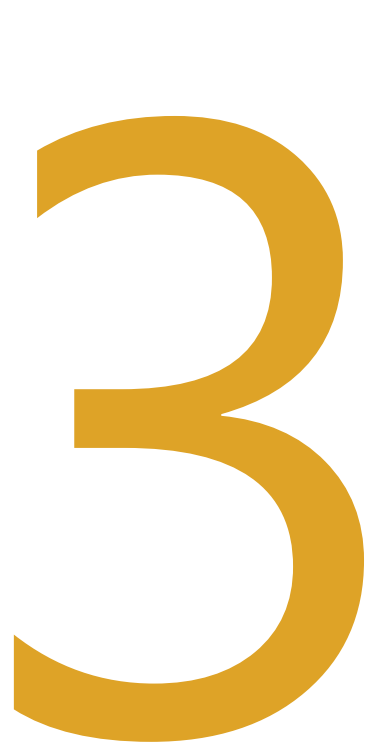

\author{
Tamar I. de Vries \\ L. Jaap Kappelle \\ Yolanda van der Graaf \\ Harold W. de Valk \\ Gert-Jan de Borst \\ Hendrik M. Nathoe \\ Frank L.J. Visseren \\ Jan Westerink;
}

on behalf of the SMART study group

Acta Diabetol. 2019;56(4):431-440. 


\section{ABSTRACT}

\section{Objective}

To evaluate the relationship between thyroid-stimulating hormone (TSH) levels within the normal range and the risk of type 2 diabetes mellitus (T2DM) in a cohort of patients at high cardiovascular risk, and to perform a systematic review and meta-analysis of previous studies.

\section{Methods}

We included 5,542 patients without T2DM from the prospective Secondary Manifestations of ARTerial disease study with TSH levels between 0.35-5.0 mU/L without anti-thyroid medication or thyroid-hormone replacement therapy. Cox regression was used to investigate the relationship between baseline plasma TSH levels and incident T2DM. MEDLINE, EMBASE, and Cochrane were searched for prospective cohorts assessing TSH and incident T2DM. Hazard ratios (HR) from included prospective cohort studies were pooled using a random-effects model.

\section{Results}

In patients at high cardiovascular risk, higher plasma TSH levels in the normal range were not associated (HR 1.07 per mIU/L increase in TSH; 95\% confidence interval [95\% CI] 0.95 - 1.22) with an increased risk of T2DM, adjusted for age, sex, smoking, total and HDL cholesterol, and triglycerides. In the meta-analysis involving three prospective cohort studies, including the present study, including 29,791 participants with 1,930 incident events, there was no relation between plasma TSH levels in the normal range and incident T2DM (pooled HR 1.06; 95\% Cl 0.99-1.14).

\section{Conclusion}

There is no apparent relation between plasma TSH levels in the normal range and incident T2DM in patients at high cardiovascular risk. 


\section{Introduction}

It has long been recognized that diabetes mellitus and thyroid disease, both common endocrine disorders, ${ }^{1,2}$ are closely related. ${ }^{3,4}$ Type 1 diabetes mellitus and auto-immune thyroid disease are associated through common auto-immune links. ${ }^{5}$ The underlying pathophysiological mechanisms of the repeatedly reported association between thyroid dysfunction and type 2 diabetes (T2DM) have not yet been fully elucidated. ${ }^{4,6,7}$ Thyroid hormones have a large impact on glucose homeostasis, ${ }^{8}$ and both high and low thyroid hormone levels are associated with peripheral insulin resistance. ${ }^{9-11}$ Triiodothyronine $\left(T_{3}\right)$ has been shown to play a role in the protection of pancreatic island $\beta$-cells against apoptosis. ${ }^{12}$ Furthermore, treatment of hypothyroidism may improve insulin sensitivity. ${ }^{13}$ Contrarily, it has been found that patients with poor glycemic control in T2DM have higher risk of subclinical hypothyroidism, ${ }^{14}$ possibly due to a stimulatory effect of higher leptin levels on the hypothalamic-pituitary-thyroid axis..$^{15}$ Thus, the association between thyroid function and T2DM is bidirectional and subject to complex and interdependent interactions. Besides the association between thyroid dysfunction, in particular hypothyroidism, and $\mathrm{T}_{2} \mathrm{DM},{ }^{4,6,7}$ increasing plasma thyroid stimulating hormone (TSH) levels within the normal range are also associated with the prevalence of T2DM in a cross-sectional study in a general adult population in China. ${ }^{16}$ Two longitudinal studies, from the Netherlands and Korea, show conflicting results. ${ }^{17-19}$

As T2DM is a considerable risk factor for cardiovascular events and mortality, identifying patients at high risk for developing T2DM is important. This is especially the case for patients who are already at high risk for cardiovascular disease.

In the current study, we aim to evaluate the relationship between plasma TSH levels in the normal range and the risk of incident T2DM in a cohort of patients at high cardiovascular risk. Additionally, we performed a systematic review and meta-analysis of studies assessing the relation between plasma TSH levels in the normal range and incident T2DM in euthyroid patients.

\section{Methods}

\section{Cohort study}

Study design and participants

Data were used from patients enrolled in the Second Manifestations of ARTerial disease (SMART) study, an ongoing prospective cohort study at the University Medical Center Utrecht, the Netherlands. A detailed description of the study design has been published previously. ${ }^{20}$ From September 1996 onwards, patients referred to our institution with clinically manifest vascular disease (coronary heart disease, cerebrovascular disease, 
peripheral arterial disease or abdominal aortic aneurysm) or vascular risk factors (dyslipidemia, hypertension or diabetes mellitus) were asked to participate. Written informed consent was obtained from all patients. The Medical Ethics Committee of the University Medical Center Utrecht approved the study.

For the present study, data were used from 7,346 patients included between July 2003 and February 2015, as routine measurement of TSH at baseline was added to the study protocol from July 2003 onwards. Patients with diabetes mellitus at baseline $(n=1,295)$, and those receiving either thyroid hormone supplementation or anti-thyroid medication ( $n=220)$ were excluded from analysis. Patients who were lost to follow-up ( $n=31)$ before the assessment of incident T2DM in 2006 were also excluded. For data analyses on the relation between TSH levels and incident T2DM patients with TSH $<0.35 \mathrm{mU} / \mathrm{L}(\mathrm{n}=81)$ or $>5.0 \mathrm{mU} / \mathrm{L}(\mathrm{n}=177)$ were excluded, restricting the analysis to 5,542 patients with plasma TSH levels in the normal range, according to the local laboratory reference values (Figure 1).

Data collection and study definitions

After inclusion, the patients underwent a standardized vascular screening protocol consisting of a health questionnaire including medical history and risk factors, physical examination and laboratory testing.

Laboratory blood testing was performed in fasting state for blood glucose, glycated hemoglobin (HbA1c), insulin levels, total cholesterol, triglycerides, high-density lipoprotein (HDL) cholesterol, and creatinine. Low-density lipoprotein (LDL) cholesterol was calculated using the Friedewald formula. The estimated glomerular filtration rate (eGFR) was calculated using the Chronic Kidney Disease Epidemiology Collaboration (CKD-EPI) formula. ${ }^{21}$ The measurement of TSH is described in detail in Supplementary Methods 1.

Diabetes mellitus at baseline was defined as patient-reported diagnosis of either type 1 or type 2 diabetes mellitus, use of glucose-lowering medication or insulin, or a plasma glucose concentration of $\geq 7.0 \mathrm{mmol} / \mathrm{L}$ at baseline with commencement of glucoselowering therapy (including diet) within 1 year after inclusion. Metabolic syndrome was defined according to the revised National Cholesterol Education Program (NCEP-R) criteria as having at least three of the following metabolic abnormalities: waist circumference $\geq$ $102 \mathrm{~cm}$ in men or $\geq 88 \mathrm{~cm}$ in women, blood pressure $\geq 130 \mathrm{mmHg}$ systolic and/or $\geq 85$ $\mathrm{mmHg}$ diastolic and/or use of blood pressure-lowering agents, triglycerides $\geq 1.7 \mathrm{mmol} / \mathrm{L}$, $\mathrm{HDL}$-cholesterol $<0.9 \mathrm{mmol} / \mathrm{L}$ in men and $<1.1 \mathrm{mmol} / \mathrm{L}$ in women, fasting glucose $\geq 5.6$ $\mathrm{mmol} / \mathrm{L} .{ }^{22}$ 
Figure 1. Flowchart of selection of study population

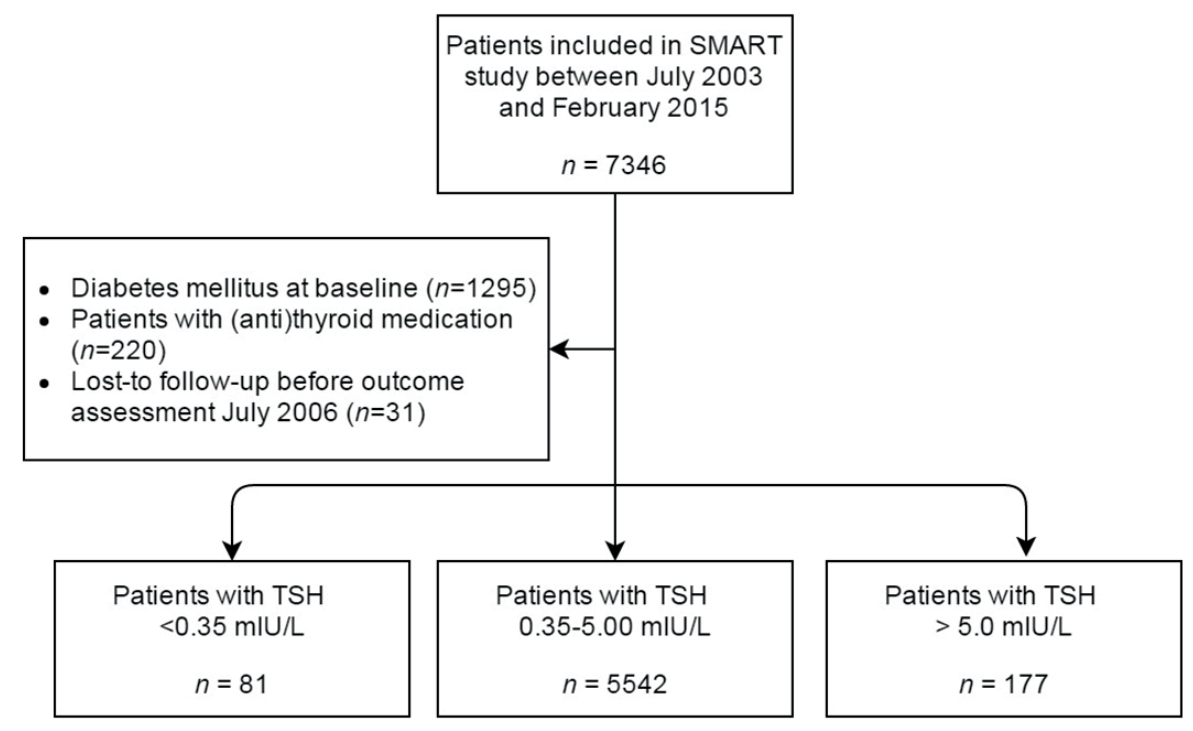

\section{Outcome assessment}

The outcome of interest in this study was incident T2DM. As this outcome was not assessed prior to July 2006, all patients without diabetes mellitus at baseline who were included in the study before July 2006 received a questionnaire in late 2006 to assess the occurrence of T2DM after study inclusion. After 2006, the occurrence of incident T2DM was biannually assessed through questionnaires. The presence of T2DM as an outcome measure was defined as either a self-reported diagnosis and/or the use of glucose-lowering agents. Patients who reported new-onset T2DM were sent an additional questionnaire for confirmation and detailed information of the diagnosis, including the date of diagnosis, initial and current treatment, and family history of diabetes. Patients and/or their general practitioners were contacted by telephone for further information if the answers were incomplete or unclear, and non-responders were also contacted. All diabetes case events were independently evaluated by three members of the SMART study end point committee. Duration of follow-up was defined as the period between study inclusion and development of incident T2DM or death from any cause, date of loss to follow-up, or the preselected date of March $1^{\text {st }} 2015$.

\section{Data analyses}

The baseline characteristics are described per sex-pooled quartiles of plasma TSH levels, to prevent overrepresentation of female subjects in the higher quartiles. ${ }^{23}$ Baseline data are presented as number and percentage for categorical variables, mean \pm standard deviation (SD) for normally distributed variables or median with interquartile range in case of a skewed distribution 
Cox proportional hazard models were fitted to estimate hazard ratios (HR) with 95\% confidence intervals $(95 \% \mathrm{Cl})$ for plasma TSH levels as a risk factor for incident T2DM. Model I was adjusted for age and sex only, model II was additionally adjusted for smoking status, total and HDL cholesterol and triglycerides. As measures of adiposity may be in the causal pathway, ${ }^{24,25}$ these were not included in the primary analysis. Exploratory models were created additionally adjusting for other potential confounders; fasting serum glucose levels, BMI, SBP, the use of lipid lowering medication, and the use of blood-pressure lowering medication. The assumption of proportionality was visually checked by plotting Schoenfeld residuals; linearity of the relation between TSH and risk of T2DM was assessed with restricted cubic splines (Supplementary Figure S1). Additionally, the same models were used to compare the plasma TSH levels as sex-pooled quartiles compared to the lowest quartile.

To investigate whether the relation between TSH and incident T2DM was modified by age, sex, or the presence of metabolic syndrome, interaction was tested between these variables and TSH for the risk of incident T2DM. A p-value of $<0.05$ was considered statistically significant.

To improve statistical accuracy, missing values for potential confounders or effect modifiers (smoking status $(n=36)$, SBP $(n=5)$, total cholesterol $(n=15)$, HDL-cholesterol $(n=20)$, triglycerides ( $n=18$ ), and fasting serum blood glucose $(n=28)$ ) were completed in the dataset also by single regression imputation. ${ }^{26}$

All statistical analysis was conducted using the statistical package $R$ for Windows ( $R$ Foundation for Statistical Computing, Vienna, Austria).

\section{Systematic review and meta-analysis}

The meta-analysis was conducted in accordance with the Preferred Reporting Items for Systematic Reviews and Meta-analyses (PRISMA) (Online Supplement with publication). We searched PubMed, Embase and the Cochrane Library from January 1 15t, 1995 to October $25^{\text {th }}, 2017$, using search terms related to TSH levels and incident T2DM (Supplementary Table 1). References of all eligible studies were searched for additional relevant studies. Mendeley Desktop (version 1.14) was used to merge retrieved reference and eliminate duplicates.

Studies were included that (a) identified a cohort (either as main analysis or subgroup) of participants with normal range plasma TSH levels without T2DM at baseline, (b) had a longitudinal study design, and (c) assessed the relation between baseline plasma TSH levels and the risk of incident T2DM, using measures of effect or relation (HR, odds ratio, or relative risk) with $95 \% \mathrm{Cl}$, or enough information to allow these to be calculated. All titles and abstracts, and consequently full texts were screened according to these selection criteria. Full texts were included if they met the criteria above. The methodological quality of the included studies was assessed using the Newcastle-Ottawa scale for cohort studies 
(NOS). ${ }^{27}$ The study characteristics (name of first author, year of publication, country, study cohort, number of participants, sex distribution, mean age, duration of follow-up, number of events, reference range plasma TSH levels, and confounding variables used in the analysis) and fully adjusted $\mathrm{HR}$ and $95 \% \mathrm{Cl}$ were extracted from the full text of the included articles. All literature screening and data extraction was performed by two independent reviewers (TV and JW); discrepancies were resolved by discussion with a third author (FV). Data analyses

The statistical analysis was performed using Review Manager (RevMan [Computer program]. Version 5.3. Copenhagen: The Nordic Cochrane Centre, The Cochrane Collaboration, 2014). The heterogeneity between the included studies was measured using the $\mathrm{I}^{2}$ statistic. ${ }^{28}$ Pooled estimates were obtained with the fully adjusted $\mathrm{HR}$ with $95 \% \mathrm{Cl}$ of the included studies, using a random-effects model as a random-effects model allows the overall effect to vary across studies. ${ }^{28,29}$ The results of the present study were also included in the pooled estimates.

\section{Results}

\section{Cohort study}

The baseline characteristics of the patients stratified for sex-pooled quartiles are presented in Table 1. The mean age of the study population was $56 \pm 12$ years, $65 \%$ of the participants were male, $27 \%$ was a current smoker at study inclusion, and $67 \%$ had a history of clinically manifest vascular disease.

\section{Plasma TSH level as a risk factor for incident T2DM}

After a median follow-up of 5.5 years (interquartile range 2.9 - 8.3) and a total followup of 31,087 person-years, there were 289 cases of T2DM (incidence rate: 9.3 per 1,000 person-years, $95 \% \mathrm{Cl} 8.3-10.4)$ in patients with $\mathrm{TSH}$ levels in the normal range. The baseline plasma TSH level did not have a significant relationship with incident T2DM (HR 1.07; $95 \% \mathrm{Cl} 0.95$ - 1.22 adjusted for age, sex, current smoking, total and HDL cholesterol, and triglycerides) (Table 2). In the exploratory models, the risk estimates did not change meaningfully (data not shown), When looking at the quartiles of baseline plasma TSH levels, there was also no significant difference between quartiles, with a fully adjusted HR of 1.07 (95\% Cl $0.77-1.48)$ for the highest compared to the lowest quartile (Table 2). Age, sex, or the presence of metabolic syndrome did not significantly modify the relation between plasma TSH levels and incident T2DM (interaction $p$-values $0.66,0.73$, and 0.21 respectively). 
Table 1. Patient characteristics according to sex-pooled TSH quartiles

\begin{tabular}{|c|c|c|c|c|}
\hline & Quartile 1 & Quartile 2 & Quartile 3 & Quartile 4 \\
\hline TSH range, men (mU/L) & $0.35-1.19$ & $1.20-1.60$ & $1.61-2.20$ & $2.21-5.00$ \\
\hline TSH range, women (mU/L) & $0.35-1.26$ & $1.27-1.80$ & $1.82-2.50$ & $2.51-5.00$ \\
\hline & $\mathrm{n}=1389$ & $n=1538$ & $n=1282$ & $n=1333$ \\
\hline Male sex & $899(65 \%)$ & 977 (64\%) & $836(65 \%)$ & 877 (66\%) \\
\hline Age (years) & $56 \pm 12$ & $55 \pm 12$ & $56 \pm 12$ & $57 \pm 12$ \\
\hline Body mass index $\left(\mathrm{kg} / \mathrm{m}^{2}\right)$ & $27 \pm 4$ & $26 \pm 4$ & $27 \pm 4$ & $27 \pm 4$ \\
\hline Systolic blood pressure (mmHg) & $140 \pm 21$ & $139 \pm 22$ & $140 \pm 22$ & $141 \pm 21$ \\
\hline Current smoker & 449 (32\%) & 475 (31\%) & 287 (22\%) & 291 (22\%) \\
\hline
\end{tabular}

\section{Laboratory values}

Glucose (mmol/L)

$\begin{array}{llll}5.7 \pm 0.7 & 5.7 \pm 0.6 & 5.6 \pm 0.7 & 5.7 \pm 0.7 \\ 5.6 \pm 0.4 & 5.5 \pm 0.4 & 5.6 \pm 0.4 & 5.6 \pm 0.4 \\ 9(6-12) & 8(6-13) & 9(6-13) & 9(6-14) \\ 4.9 \pm 1.3 & 4.9 \pm 1.3 & 5.0 \pm 1.4 & 5.0 \pm 1.3 \\ 1.3 \pm 0.4 & 1.3 \pm 0.4 & 1.3 \pm 0.4 & 1.3 \pm 0.4 \\ 2.9 \pm 1.1 & 2.9 \pm 1.1 & 3.0 \pm 1.2 & 3.0 \pm 1.2 \\ 1.2(0.9-1.8) & 1.2(0.9-1.8) & 1.3(0.9-1.8) & 1.3(0.9-1.9) \\ 82 \pm 17 & 81 \pm 18 & 80 \pm 17 & 78 \pm 18\end{array}$

\section{Medical history}

$\begin{array}{lllll}\text { Clinically manifest vascular disease } & 972(70 \%) & 1034(67 \%) & 840(66 \%) & 879(66 \%) \\ \text { Coronary heart disease } & 622(45 \%) & 657(43 \%) & 516(40 \%) & 535(40 \%) \\ \text { Cerebrovascular disease } & 285(21 \%) & 303(20 \%) & 242(19 \%) & 264(20 \%) \\ \text { Peripheral vascular disease } & 136(10 \%) & 128(8 \%) & 114(9 \%) & 131(10 \%) \\ \text { Metabolic syndrome* } & 620(45 \%) & 598(39 \%) & 553(43 \%) & 590(44 \%)\end{array}$

\section{Medication use}

$\begin{array}{lllll}\text { Lipid lowering medication } & 874(63 \%) & 928(60 \%) & 784(61 \%) & 792(59 \%) \\ \text { Blood pressure lowering medication } & 979(70 \%) & 1069(70 \%) & 836(65 \%) & 895(67 \%)\end{array}$

Abbreviatons: $T S H=$ thyroid stimulating hormone; HbA1C = Glycated hemoglobin A1c; $H D L=$ high-density lipoprotein; $L D L=$ low-density lipoprotein; eGFR = estimated glomerular filtration rate; CKD-EPI=Chronic Kidney Disease Epidemiology Collaboration

* According to the revised criteria of the National Cholesterol Education Program

All data in $n(\%)$, mean \pm standard deviation, or median (interquartile range) 
Table 2. Relation between normal-range TSH and incident type 2 diabetes

\section{Quartiles of baseline TSH level}

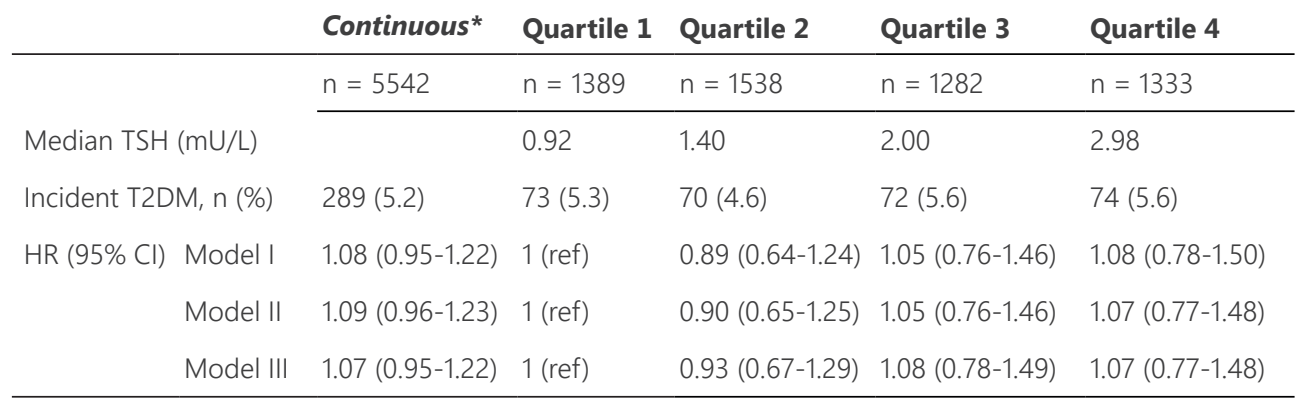

$H R=$ hazard ratios; $95 \% \mathrm{Cl}=95 \%$ confidence intervals.

Model I, crude model; Model II, adjusted for age and sex; Model III, adjusted for age, sex, current smoking, total and $\mathrm{HDL}$ cholesterol, and triglycerides. * The hazard ratio denotes the increase in risk for incident diabetes per one $\mathrm{mU} / \mathrm{L}$ rise in level of TSH within the normal range $(0.35-5.0 \mathrm{mU} / \mathrm{L})$

\section{Systematic review and meta-analysis}

The search initially yielded 1,361 results. After screening of title, abstract and full text, 3 articles based on 2 unique studies were eligible for inclusion (Supplementary Figure S2). ${ }^{17-19}$ We included the 2 studies in the meta-analysis, using the most recent article of the unique studies. ${ }^{17,19}$ Thus, we meta-analyzed the results from 3 studies including the present study. The study characteristics and methodological quality as assessed using the NOS can be found in Table 3 and Supplementary Table S2, respectively. The three studies included a total of 29,791 participants, with a total of 1,930 events of incident T2DM.

The pooled HR for the relation between continuous TSH levels within the normal range and incident T2DM was $1.06(95 \% \mathrm{Cl}$ 0.99-1.14) (Figure 2). Moderate statistical heterogeneity was observed, $1^{2}=38 \%$. 


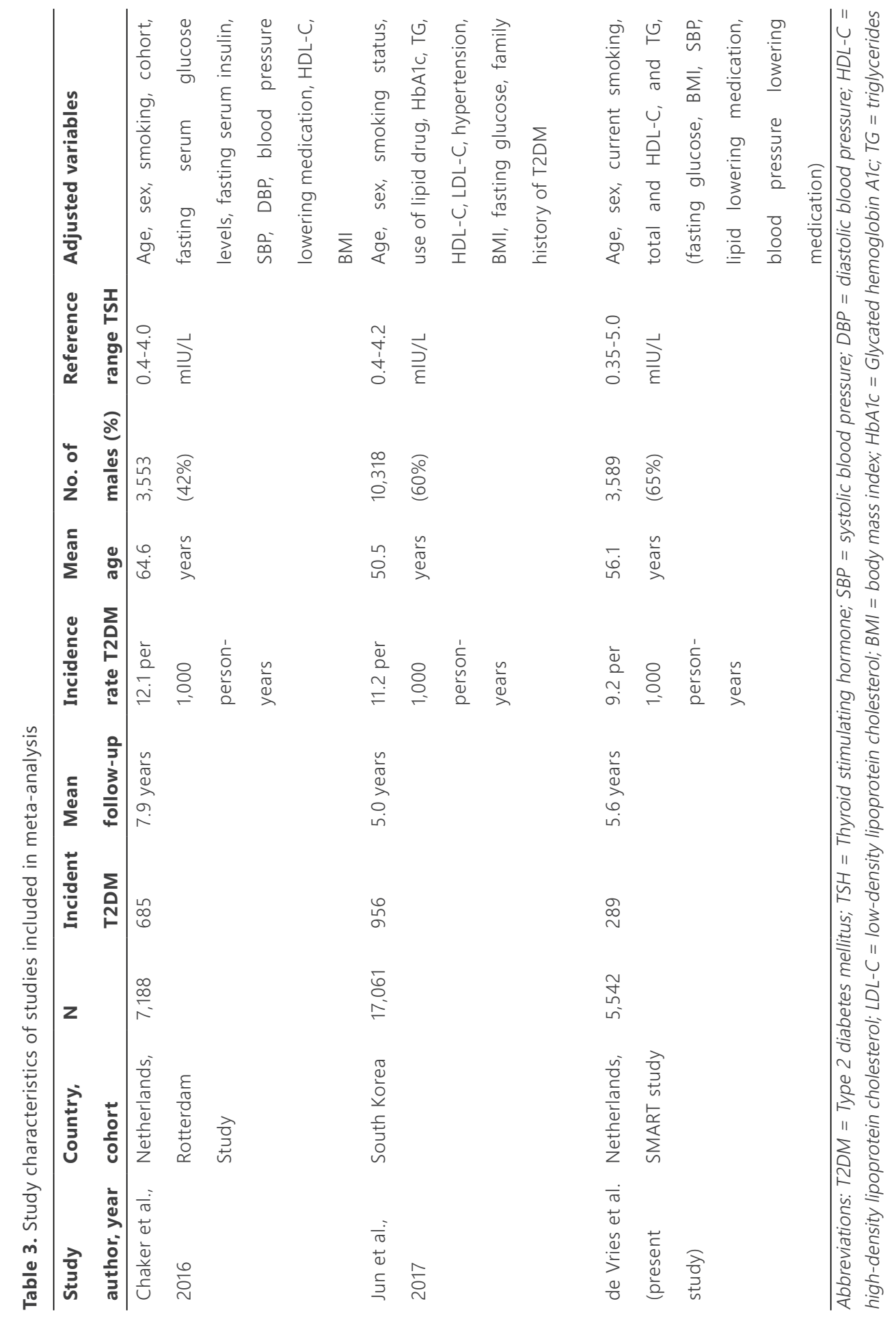


Figure 2. Meta-analysis of reported hazard ratios for the association between plasma TSH levels and incident T2DM, with the pooled hazard ratio.

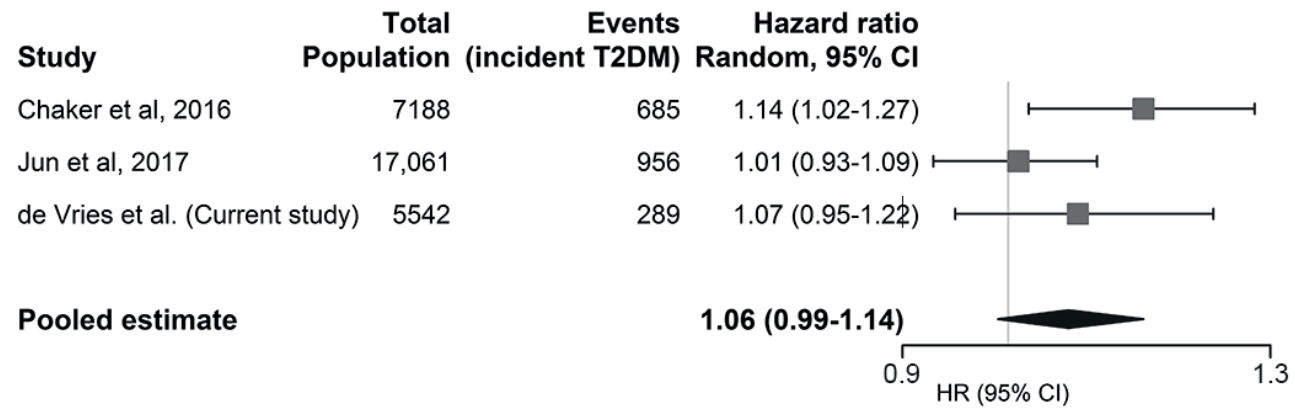

\section{Discussion}

In this prospective cohort study, no relation was found between plasma TSH levels within the normal range and the risk of incident T2DM in patients at high risk for cardiovascular disease. In addition, pooled results from the systematic meta-analysis of 3 studies, including the present study, showed no relation between plasma TSH levels and incident T2DM.

The results of the present study are in line with the results of the cohort study from South Korea, where the baseline plasma TSH levels in the normal range were not associated with increased risk of incident T2DM. ${ }^{18}$ Interestingly, the authors of that study found an association between an increase in plasma TSH levels over time and incident T2DM. However, the question is whether this represents a causal relation or an opposite association, with plasma TSH levels increasing due to increasing insulin resistance in developing T2DM. ${ }^{30}$

The results of the present study are, however, in contrast with the results from the Rotterdam Study, which found a higher risk for incident T2DM in patients with higher TSH levels in the normal range. ${ }^{17}$

A notable difference between the studies is the used reference range for euthyroidism (0.4-4.2 $\mathrm{mIU} / \mathrm{L}$ in the Korean study, ${ }^{19}$ 0.4-4.0 mIU/L in the Rotterdam Study, ${ }^{17}$ and 0.35-5.0 $\mathrm{mIU} / \mathrm{L}$ in the present study). Of note is that the reference range used in the Korean study is based on Western values, ${ }^{23}$ while a recent Korean study showed that the reference value of plasma TSH in the Korean population is higher (0.62-6.68 mIU/L in 6,564 participants). ${ }^{31}$ In order to exclude the possibility that the different reference range in the present study explains the differences in the results, we repeated our analyses using the reference value of 0.4-4.2 mIU/L. This analysis did not change the risk estimates meaningfully (data not shown). 
Furthermore, the domains the study populations were taken from are different. The Rotterdam Study included participants among all inhabitants aged 55 years and older in one district in the city Rotterdam. The Korean Study included participants among people participating in a yearly health check-up program in a single center. Thus, these populations are very different from the population in our study, which consists of patients with high cardiovascular risk, referred to a secondary and tertiary health care center. The Rotterdam Study has a higher proportion of women in the study population (58\%) compared with the Korean study (40\%) and the present study (35\%). In the general population women on average have higher plasma TSH values than men. ${ }^{23}$ Furthermore, the Rotterdam Study had a higher mean age (65 years), compared to the Korean study (51 years) and the present study (56 years). It is well-recognized that plasma TSH levels increase with age. ${ }^{23}$ However, the primary analysis was adjusted for both age and sex, and interaction analysis in both the present study and the Rotterdam Study showed that age and sex were not important effect modifiers. ${ }^{17}$ Additionally, compared to the Korean study, the participants in the present study used more lipid lowering medication, independently a risk factor for incident T2DM (data not available for the Rotterdam Study). ${ }^{32}$ However, adjustments for lipid lowering medication in an exploratory analysis in the present study had no significant effect on the risk estimate. Finally, the present study consists mostly of participants from Caucasian descent, whereas the participants from the Korean study are mainly from Asian descent. There are differences between Caucasian and Asian people with regards to insulin resistance and diabetes, ${ }^{33}$ body weight distribution, ${ }^{34}$ and reference TSH values. ${ }^{31}$ The ethnicity of the patients of the Rotterdam Study has not been reported. ${ }^{17}$ In a recent Mendelian randomization study of 69,033 euthyroid individuals with 12,171 cases of T2DM, no evidence for a causal relation was found between 20 genetic variants for TSH levels, and 4 variants for free thyroid hormone (fT4) levels, and insulin resistance and T2DM (Odds ratio 0.91 per SD TSH increase; 95\% Cl 0.78-1.07). ${ }^{35}$ As Mendelian randomization studies are at a low risk of confounding and reverse causality, it is a good method to ascertain causality of observational associations. ${ }^{36}$ However, the selected loci only explained $5.64 \%$ of the total variation in TSH concentration and only euthyroid participants were included in the analysis. Furthermore, plasma TSH levels are in part determined by non-genetic factors, ${ }^{37}$ which are not taken into account in a Mendelian randomization study. Therefore, it is possible this study underestimates the relation between plasma TSH levels and incident T2DM.

The combined evidence from the Mendelian randomization study and the present study and meta-analysis does not indicate a causal relationship between plasma TSH levels in the normal range and the risk of incident T2DM. It is possible that the observed association between plasma TSH levels and incident T2DM in the Rotterdam Study was due to reverse causality (i.e. insulin resistance leading to higher plasma TSH levels) ${ }^{38}$ and/or unidentified confounders. Based on this evidence, no recommendations with regards to screening of 
thyroid function in patients with high risk of developing T2DM are necessary.

Important to realize is that these results only apply to plasma TSH levels in the normal range. As thyroid dysfunction is associated with altered metabolic parameters, ${ }^{39}$ patients with thyroid dysfunction cannot be compared with euthyroid patients. Previous studies have reported an association between thyroid dysfunction and T2DM. ${ }^{40-42}$ As free thyroxine levels were not available in the present study, thyroid dysfunction was not investigated in the scope of the present study, and therefore it is not possible to make any statements about the probable relation between thyroid dysfunction and incident T2DM.

Strengths of our study include the large number of individuals from a clinically highly relevant population of patients at high risk for cardiovascular events, the long follow-up, and the extensive availability of data for possible confounders and interaction analysis.

A limitation of the study is that T2DM was only registered as an endpoint after 2006 and this information was collected retrospectively for patients included before 2006 . Patients who did not respond to the questionnaires sent in 2006 were considered as lossto follow-up, which may induce bias as this loss-to follow-up may not have been random. Additionally, the first assessment of T2DM at follow-up was based on self-reported diabetes, and was not confirmed by measuring plasma glucose levels or performing an oral glucose tolerance test. However, the patients reporting T2DM were sent an additional questionnaire asking for detailed information of the diagnosis, including medication use. Furthermore, we have no data on the levels of free thyroxine. Therefore, we could not with certainty classify all participants as euthyroid. The Rotterdam Study showed that an inverse relationship between fT4 and T2DM which we also could not investigate in the current study. ${ }^{17}$ Finally, the studies in the meta-analysis have very different study populations. Based on previous literature, and the performed sensitivity analyses and adjustments, there are no important reasons to assume that the relationship between plasma TSH levels and incident T2DM would be different in different study populations. However, it is possible that there are unknown underlying reasons that influence this relationship in different study populations.

In conclusion, the results of the current prospective cohort study in patients at high cardiovascular risk and a separate meta-analysis do not indicate a causal relation between plasma TSH levels within the normal range and incident T2DM. 


\section{References}

1. Vanderpump MPJ. The epidemiology of thyroid disease. Br Med Bull. 2011;99:39-51.

2. Centers for Disease Control and Prevention. National Diabetes Statistics Report: Estimates of Diabetes and Its Burden in the United States. Atlanta, GA; 2014.

3. Wang C. The Relationship between Type 2 Diabetes Mellitus and Related Thyroid Diseases. J Diabetes Res Egypt. 2013;2013:390534.

4. Han C, He X, Xia X, Li Y, Shi X, Shan Z, Teng W. Subclinical Hypothyroidism and Type 2 Diabetes: A Systematic Review and Meta-Analysis. PLoS One. 2015;10:e0135233.

5. Barker JM. Clinical review: Type 1 diabetes-associated autoimmunity: natural history, genetic associations, and screening. J Clin Endocrinol Metab. 2006;91:1210-1217.

6. Fleiner HF, Bjøro T, Midthjell K, Grill V, Asvold BO. Prevalence of Thyroid Dysfunction in Autoimmune and Type 2 Diabetes: The Population-Based HUNT Study in Norway. J Clin Endocrinol Metab. 2016;101:669-677.

7. Song F, Bao C, Deng M, Xu H, Fan M, Paillard-Borg S, et al. The prevalence and determinants of hypothyroidism in hospitalized patients with type 2 diabetes mellitus. Endocrine. 2017;55:179185.

8. Coller FA, Huggins CB. Effect of hyperthyroidism upon diabetes mellitus: striking improvement in diabetes mellitus from thyroidectomy. Ann Surg. 1927;86:877-884.

9. Dimitriadis G, Baker B, Marsh H, Mandarino L, Rizza R, Bergman R, et al. Effect of thyroid hormone excess on action, secretion, and metabolism of insulin in humans. Am J Physiol. 1985;248:E593601.

10. Lambadiari V, Mitrou P, Maratou E, Raptis AE, Tountas N, Raptis SA, Dimitriadis G. Thyroid hormones are positively associated with insulin resistance early in the development of type 2 diabetes. Endocrine. 2011;39:28-32.

11. Dimitriadis G, Mitrou P, Lambadiari V, Boutati E, Maratou E, Panagiotakos DB, et al. Insulin action in adipose tissue and muscle in hypothyroidism. J Clin Endocrinol Metab. 2006;91:4930-4937.

12. Verga Falzacappa C, Panacchia L, Bucci B, Stigliano A, Cavallo MG, Brunetti E, et al. 3,5,3'-triiodothyronine (T3) is a survival factor for pancreatic beta-cells undergoing apoptosis. J Cell Physiol. 2006;206:309-321.

13. Stanicka S, Vondra K, Pelikanova T, Vlcek P, Hill M, Zamrazil V. Insulin sensitivity and counterregulatory hormones in hypothyroidism and during thyroid hormone replacement therapy. Clin Chem Lab Med. 2005;43:715-720.

14. Cho JH, Kim HJ, Lee JH, Park IR, Moon JS, Yoon JS, et al. Poor glycemic control is associated with the risk of subclinical hypothyroidism in patients with type 2 diabetes mellitus. Korean J Intern Med. 2016;31:703-711.

15. Ortiga-Carvalho TM, Oliveira KJ, Soares BA, Pazos-Moura CC. The role of leptin in the regulation of TSH secretion in the fed state: in vivo and in vitro studies. J Endocrinol. 2002;174:121-125.

16. Gu Y, Li H, Bao X, Zhang Q, Liu L, Meng G, et al. The relationship between thyroid function 
and the prevalence of type 2 diabetes mellitus in euthyroid subjects. J Clin Endocrinol Metab. 2017;102:434-442.

17. Chaker L, Ligthart S, Korevaar TIM, Hofman A, Franco OH, Peeters RP, Dehghan A. Thyroid function and risk of type 2 diabetes: a population-based prospective cohort study. BMC Med. 2016;14:150.

18. Jun JE, Jee JH, Bae JC, Jin SM, Hur KY, Lee MK, et al. Association between Changes in Thyroid Hormones and Incident Type 2 Diabetes: A Seven-Year Longitudinal Study. Thyroid. 2017;27:2938.

19. Jun JE, Jin SM, Jee JH, Bae JC, Hur KY, Lee MK, et al. TSH increment and the risk of incident type 2 diabetes mellitus in euthyroid subjects. Endocrine. 2017;55:944-953.

20. Simons PC, Algra A, Laak MF van de, Grobbee DE, Graaf $Y$ van der. Second manifestations of ARTerial disease (SMART) study: rationale and design. Eur J Endocrinol. 1999;15:773-781.

21. Levey AS, Stevens LA, Schmid CH, Zhang YL, Castro AF, Feldman HI, et al. A new equation to estimate glomerular filtration rate. Ann Intern Med. 2009;150:604-612.

22. Grundy SM, Cleeman JI, Daniels SR, Donato K, Eckel RH, Franklin B et al. Diagnosis and management of the metabolic syndrome: An American Heart Association/National Heart, Lung, and Blood Institute Scientific Statement. Executive summary. Cardiol Rev. 2005;13:322-327.

23. Hollowell JG, Staehling NW, Flanders WD, Hannon WH, Gunter EW, Spencer CA, Braverman LE. Serum TSH, T(4), and thyroid antibodies in the United States population (1988 to 1994): National Health and Nutrition Examination Survey (NHANES III). J Clin Endocrinol Metab. 2002;87:489499.

24. Fox CS, Pencina MJ, D'Agostino RB, Murabito JM, Seely EW, Pearce EN, Vasan RS. Relations of thyroid function to body weight: cross-sectional and longitudinal observations in a communitybased sample. Arch Intern Med. 2008;168:587-592.

25. Franssens BT, Graaf Y van der, Kappelle LJ, Westerink J, Borst GJ de, Cramer MJ, Visseren FLJ, SMART Study Group. Body weight, metabolic dysfunction, and risk of type 2 diabetes in patients at high risk for cardiovascular events or with manifest cardiovascular disease: a cohort study. Diabetes Care. 2015;38:1945-1951.

26. Zhang Z. Missing data imputation: focusing on single imputation. Ann Transl Med. 2016.

27. Wells G, Shea B, O'Connell D, Peterson J, Welch V, Losos M, Tugwell P. The Newcastle-Ottawa Scale (NOS) for assessing the quality of nonrandomised studies in meta-analyses. http://www. ohri.ca/programs/clinical_epidemiology/oxford.asp [Accessed 11th April 2017]

28. Higgins JPT, Thompson SG, Deeks JJ, Altman DG. Measuring inconsistency in meta-analyses. Br Med J. 2003;327:557-560.

29. Brockwell SE, Gordon IR. A comparison of statistical methods for meta-analysis. Stat Med. 2001;20:825-840.

30. Hage M, Zantout MS, Azar ST. Thyroid disorders and diabetes mellitus. J Thyroid Res. 2011;2011:439463.

31. Kim WG, Kim WB, Woo G, Kim H, Cho Y, Kim TY, K, et al. Thyroid Stimulating Hormone Reference 
Range and Prevalence of Thyroid Dysfunction in the Korean Population: Korea National Health and Nutrition Examination Survey 2013 to 2015. Endocrinol Metab. 2017;32:106-114.

32. Casula M, Mozzanica F, Scotti L, Tragni E, Pirillo A, Corrao G, Catapano AL. Statin use and risk of new-onset diabetes: A meta-analysis of observational studies. Nutr Metab Cardiovasc Dis. 2017;27:396-406.

33. Spanakis EK, Golden SH. Race/ethnic difference in diabetes and diabetic complications. Curr Diab Rep. 2013;13:814-823.

34. Heymsfield SB, Peterson CM, Thomas DM, Heo M, Schuna JMJ. Why are there race/ethnic differences in adult body mass index-adiposity relationships? A quantitative critical review. Obes Rev. 2016;17:262-275.

35. Bos MM, Smit RA, Trompet S, Heemst D van, Noordam R. Thyroid signaling, insulin resistance and type 2 diabetes mellitus: a Mendelian randomization study. J Clin Endocrinol Metab. 2017;102(6):1960-1970.

36. Lawlor DA, Harbord RM, Sterne JAC, Timpson N, Davey Smith G, Thimpson N, Smith GD. Mendelian randomization: using genes as instruments for making causal inferences in epidemiology. Stat Med. 2008;27:1133-1163.

37. Hansen PS, Brix TH, Sorensen TIA, Kyvik KO, Hegedus L. Major genetic influence on the regulation of the pituitary-thyroid axis: a study of healthy Danish twins. J Clin Endocrinol Metab. 2004;89:1181-1187.

38. Sandhu MS, Debenham SL, Barroso I, Loos RJF. Mendelian randomisation studies of type 2 diabetes: future prospects. Diabetologia. 2008;51:211-213.

39. Iwen KA, Schroder E, Brabant G. Thyroid hormones and the metabolic syndrome. Eur Thyroid J. 2013;2:83-92.

40. Gronich N, Deftereos SN, Lavi I, Persidis AS, Abernethy DR, Rennert G. Hypothyroidism is a Risk Factor for New-Onset Diabetes: A Cohort Study. Diabetes Care. 2015;38:1657-1664.

41. Brandt F, Thvilum M, Almind D, Christensen K, Green A, Hegedus L, Brix TH. Morbidity before and after the diagnosis of hyperthyroidism: a nationwide register-based study. PLoS One. 2013;8:e66711.

42. Thvilum M, Brandt F, Almind D, Christensen K, Brix TH, Hegedus L. Type and extent of somatic morbidity before and after the diagnosis of hypothyroidism. a nationwide register study. PLoS One. 2013;8:e75789. 


\section{Supplementary Material}

\section{Supplementary Methods 1. Measurement of TSH}

Before November 2006, TSH was quantified using a third-generation assay on a Centaur analyzer (Bayer, Germany). This analyzer had an interassay variation of $0.22 \mathrm{mU} / \mathrm{l}(6 \%), 4.5 \mathrm{mU} / \mathrm{l}(5.5 \%), 16.5 \mathrm{mU} / \mathrm{l}(5.2 \%)$. The functional sensitivity of the Centaur analyzer was $0.02 \mathrm{mU} / \mathrm{l}$ with an interassay imprecision of $20 \%$. Starting December 2006, TSH was measured by a third-generation assay on a DXi analyzer (Beckman Coulter, Woerden, The Netherlands), an analyzer with an interassay variation of 4-8\%, and a functional sensitivity of $0.015 \mathrm{mU} / \mathrm{I}$ with an interassay imprecision of $20 \%$. Correlation between the two analyzers was $r=0.9991(n=69)$, with an intercept of $-0.05 \mathrm{mU} / \mathrm{l}(95 \% \mathrm{Cl} 0.22$ to 0.12$)$ and a slope of $1.04(95 \% \mathrm{Cl}$ 1.029-1.052) (range 0-95 mU/L). 
Supplementary Figure S1. (A) Schoenfeld residuals plot for the proportional hazards assumption ( $\mathrm{X}$-axis shows the follow-up time in days, $\mathrm{Y}$-axis shows the log hazard ratio for the relation between plasma TSH and incident type 2 diabetes [T2DM]); and (B) Spline for the relationship between TSH in the normal range and incident T2DM

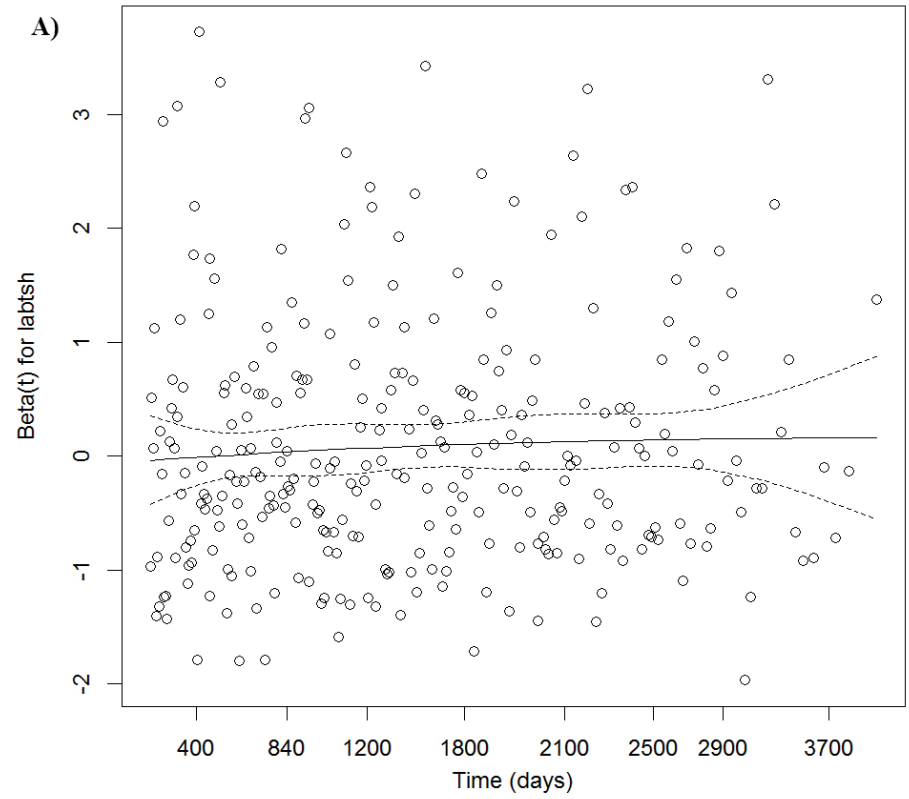

Relationship TSH and incident T2DM

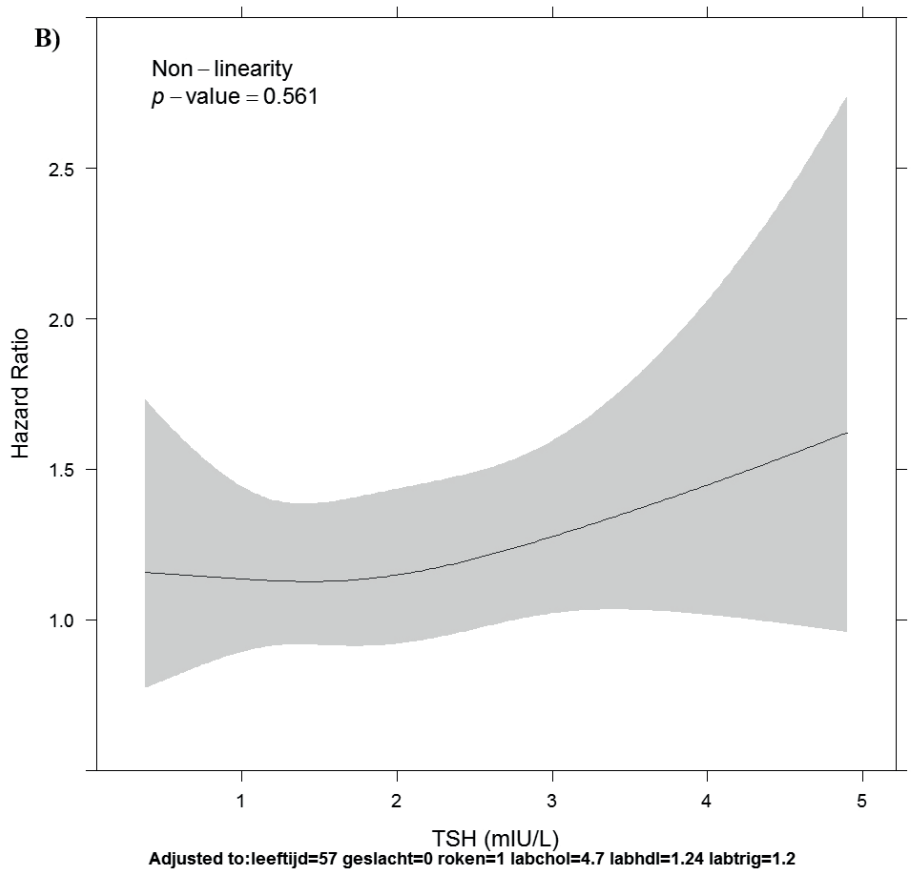




\section{Supplementary Table S1. Search strategy for meta-analysis}

\begin{tabular}{|c|c|}
\hline PubMed & $\begin{array}{l}((((((((()((((\text { TSH[Title/Abstract]) OR Thyroid-Stimulating Hormone[Title/Abstract]) OR Thyroid } \\
\text { Stimulating Hormone[Title/Abstract]) OR thyrotropin[Title/Abstract]) OR Thyreotropin[Title/ } \\
\text { Abstract]) OR Thyroid hormone*[Title/Abstract]) OR Thyroid function*[Title/Abstract]) } \\
\text { OR euthyroid*[Title/Abstract])) OR ((“Thyrotropin"[Mesh]) OR "Thyroid Function } \\
\text { Tests"[Mesh]))))) AND ((((((((((((((((T2DM[Title/Abstract]) OR type } 2 \text { diabetes[Title/Abstract]) } \\
\text { OR NIDDM[Title/Abstract]) OR T2DM[Title/Abstract]) OR Maturity-Onset Diabetes[Title/ } \\
\text { Abstract]) OR Maturity Onset Diabetes[Title/Abstract]) OR Adult-Onset Diabetes[Title/ } \\
\text { Abstract]) OR Adult Onset Diabetes[Title/Abstract]) OR Non Insulin Dependent } \\
\text { diabetes[Title/Abstract]) OR Non-Insulin Dependent diabetes[Title/Abstract]) OR Non- } \\
\text { Insulin-Dependent diabetes[Title/Abstract]) OR Noninsulin-dependent diabetes[Title/ } \\
\text { Abstract]) OR Noninsulin Dependent diabetes[Title/Abstract]) OR slow onset diabetes[Title/ } \\
\text { Abstract]) OR slow-onset diabetes[Title/Abstract]) OR "Diabetes Mellitus, Type 2"[Mesh]))) }\end{array}$ \\
\hline Embase & $\begin{array}{l}\text { ('maturity-onset diabetes':ab,ti OR 'diabetes, noninsulin-dependent':ab,ti OR 'diabetes, } \\
\text { adult-onset':ab,ti OR 'adult-onset diabetes ':ab,ti OR 'diabetes, adult onset':ab,ti OR } \\
\text { 'diabetes, maturity-onset':ab,ti OR 'diabetes, maturity onset':ab,ti OR 'diabetes, non insulin } \\
\text { dependent':ab,ti OR 'diabetes, non-insulin-dependent':ab,ti OR 'non-insulin-dependent } \\
\text { diabetes ':ab,ti OR 'diabetes, noninsulin dependent':ab,ti OR 'diabetes, slow-onset':ab,ti } \\
\text { OR 'diabetes, slow onset':ab,ti OR 'slow-onset diabetes ':ab,ti OR 'diabetes, type ii':ab,ti } \\
\text { OR 'maturity-onset diabetes ':ab,ti OR 'maturity onset diabetes ':ab,ti OR 'type } 2 \text { diabetes } \\
\text { ':ab,ti OR 'noninsulin-dependent diabetes ':ab,ti OR 'type } 2 \text { diabetes':ab,ti OR 'non insulin } \\
\text { dependent diabetes mellitus'/exp) } \\
\text { AND } \\
\text { ('TSH':ab,ti OR 'Thyroid-Stimulating Hormone':ab,ti OR 'Thyroid Stimulating Hormone':ab,ti } \\
\text { OR 'thyrotropin':ab,ti OR 'Thyreotropin':ab,ti OR 'Thyroid hormone*':ab,ti OR 'Thyroid } \\
\text { function`':ab,ti OR 'euthyroid':ab,ti OR 'euthyroidism':ab,ti OR 'euthyroidism'/exp OR } \\
\text { 'thyrotropin'/exp) }\end{array}$ \\
\hline $\begin{array}{l}\text { Cochrane } \\
\text { Library }\end{array}$ & $\begin{array}{l}\text { ('maturity-onset diabetes':ab,ti or 'diabetes mellitus, noninsulin-dependent':ab,ti or } \\
\text { 'diabetes mellitus, adult-onset':ab,ti or 'adult-onset diabetes mellitus':ab,ti or 'diabetes } \\
\text { mellitus, adult onset':ab,ti or 'diabetes mellitus, maturity-onset':ab,ti or 'diabetes mellitus, } \\
\text { maturity onset':ab,ti or 'diabetes mellitus, non insulin dependent':ab,ti or 'diabetes mellitus, } \\
\text { non-insulin-dependent':ab,ti or 'non-insulin-dependent diabetes mellitus':ab,ti or 'diabetes } \\
\text { mellitus, noninsulin dependent':ab,ti or 'diabetes mellitus, slow-onset':ab,ti or 'diabetes } \\
\text { mellitus, slow onset':ab,ti or 'slow-onset diabetes mellitus':ab,ti or 'diabetes mellitus, } \\
\text { stable':ab,ti or 'stable diabetes mellitus':ab,ti or 'diabetes mellitus, type ii':ab,ti or 'maturity- } \\
\text { onset diabetes mellitus':ab,ti or 'maturity onset diabetes mellitus':ab,ti or 'type } 2 \text { diabetes } \\
\text { mellitus':ab,ti or 'noninsulin-dependent diabetes mellitus':ab,ti or 'type } 2 \text { diabetes':ab,ti) OR } \\
\text { (MeSH descriptor: [Diabetes Mellitus, Type 2])) } \\
\text { AND } \\
\text { ('TSH':ab,ti or 'Thyroid-Stimulating Hormone':ab,ti or 'Thyroid Stimulating Hormone':ab,ti } \\
\text { or 'thyrotropin':ab,ti or 'Thyreotropin':ab,ti or 'Thyroid hormone*':ab,ti or 'Thyroid } \\
\text { function*':ab,ti or 'euthyroid':ab,ti or 'euthyroidism':ab,ti) OR (MeSH descriptor: [Thyrotropin] } \\
\text { OR [Thyroid Function Tests])) }\end{array}$ \\
\hline
\end{tabular}


Supplementary Figure S2. Study selection for meta-analysis

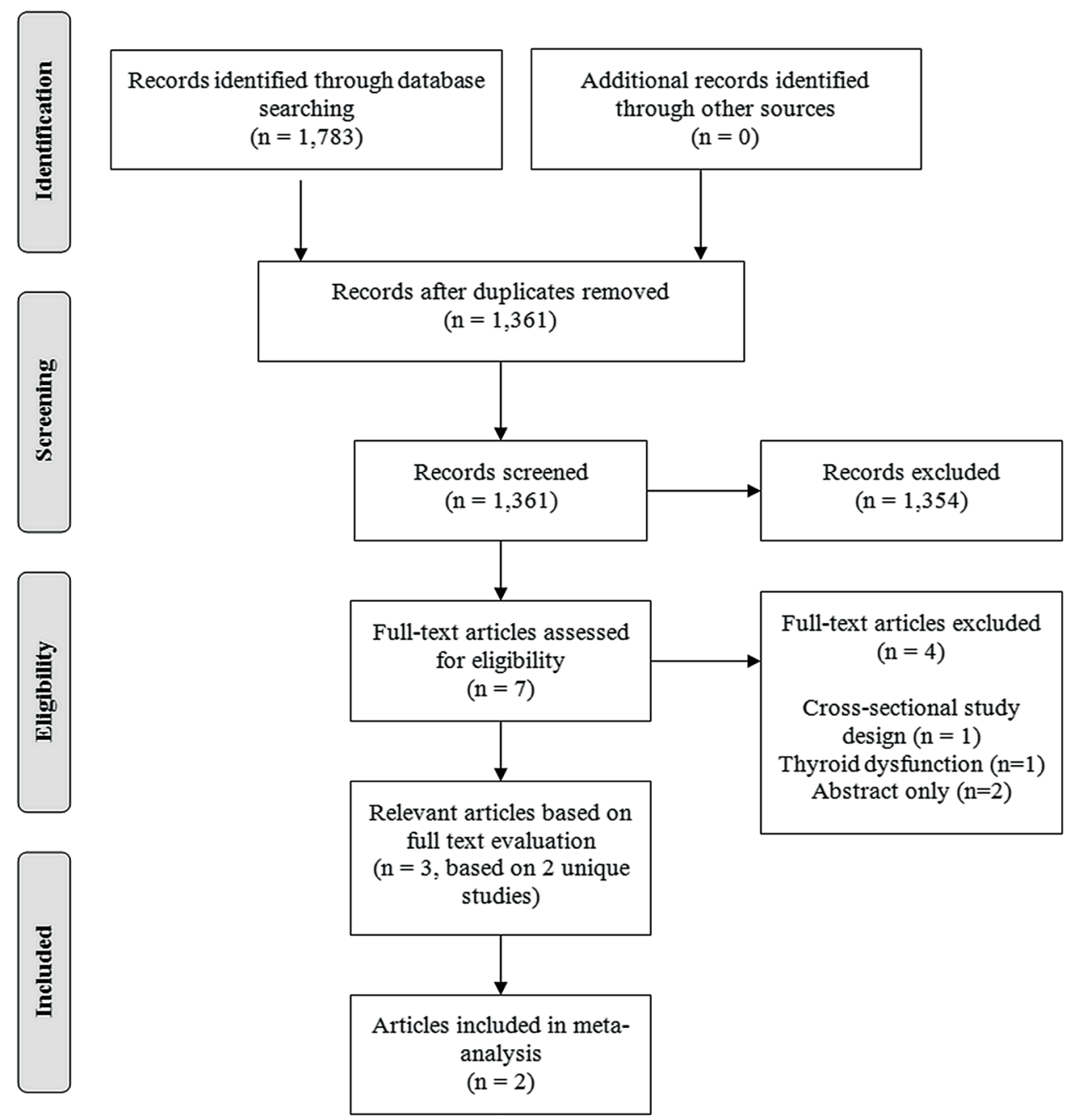


Supplementary Table S2. Quality assessment of studies included in meta-analysis

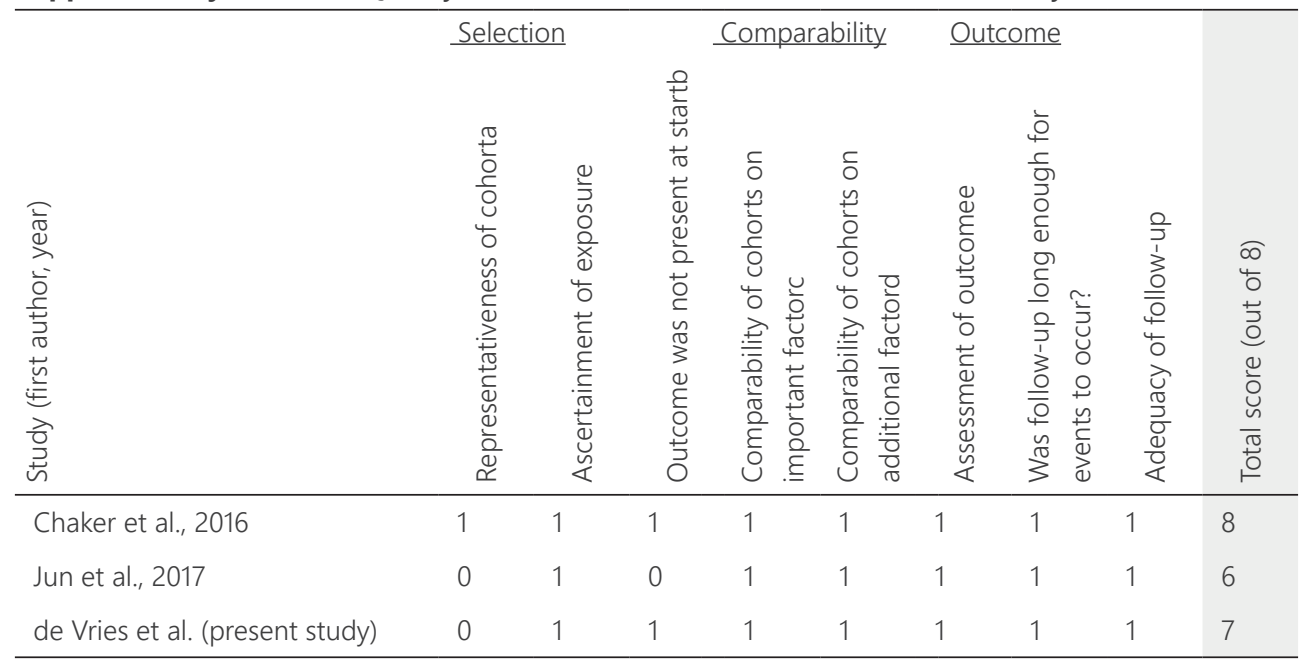

a Adapted from the NOS. As plasma TSH level is a continuous level, there is no exposed vs unexposed cohort. No point if single center cohort.

$b$ No point if relied on self-report or if method of ascertainment that outcome was not present at inclusion was not clearly described.

c Must control for age and gender most importantly.

$d$ Must control for smoking status as this is an important confounder.

e Assessment of type 2 diabetes must be based on the official criteria of the American Diabetes Association. 


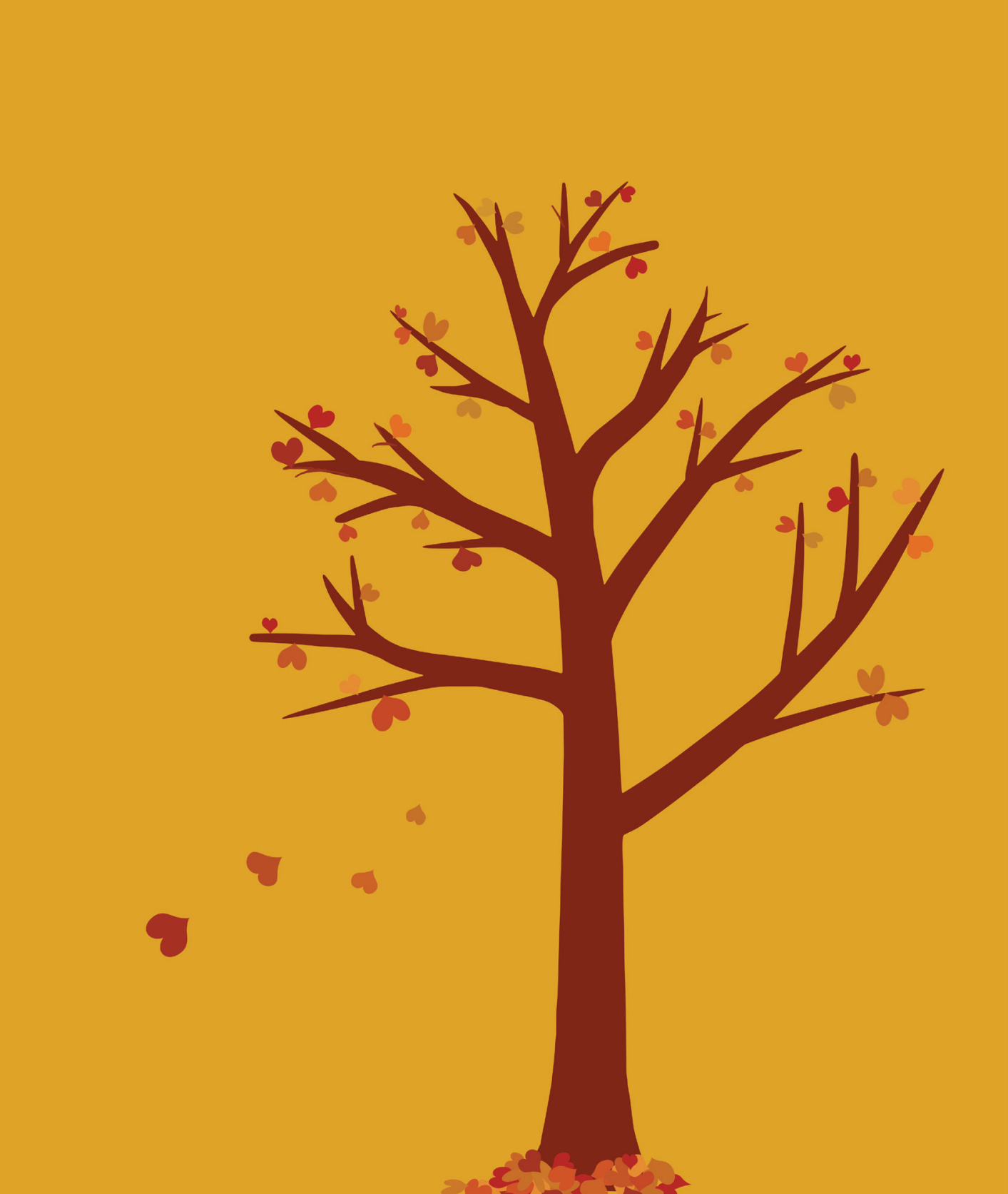




\section{Normal-range thyroid-stimulating hormone levels and cardiovascular events and mortality in type 2 diabetes}

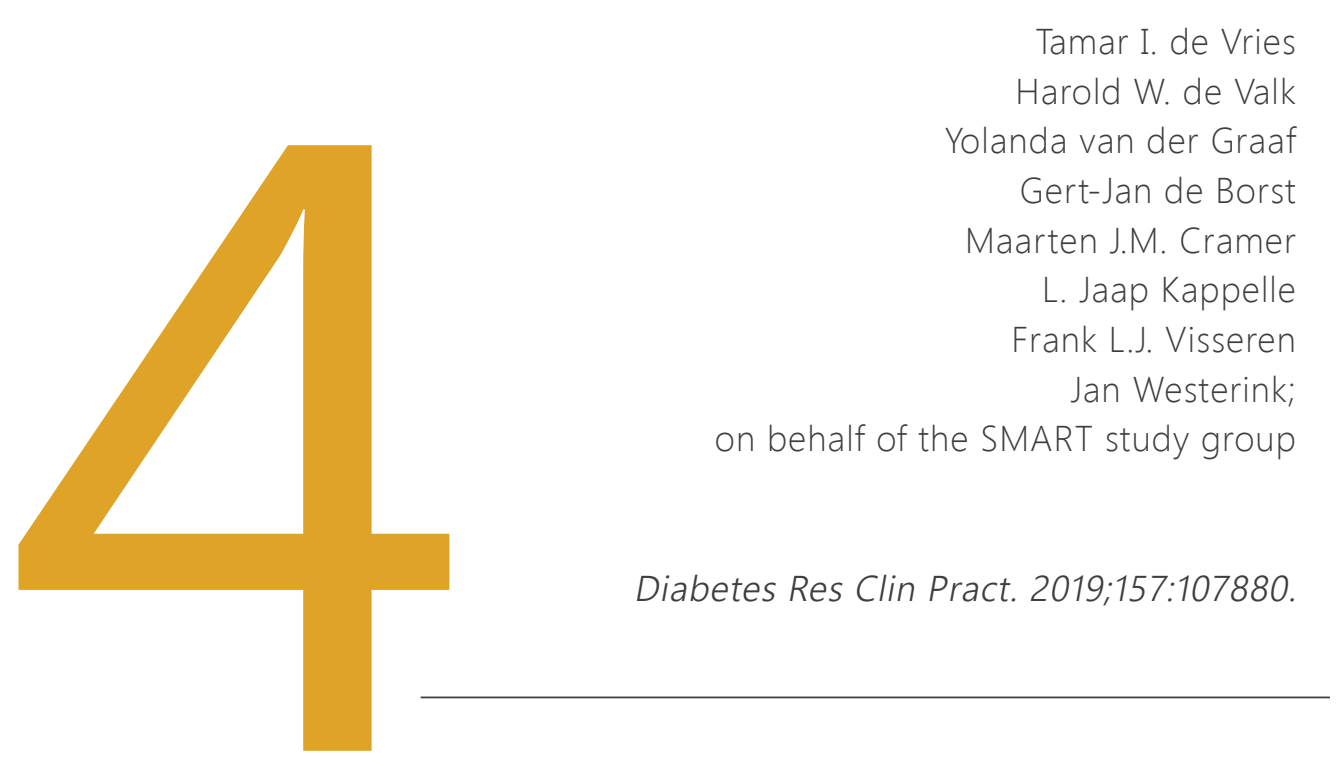




\section{ABSTRACT}

\section{Aims}

Thyroid dysfunction is a risk factor for cardiovascular disease. Whether thyroid function within the normal range is a risk factor for cardiovascular disease remains uncertain. The aim of this study is to evaluate whether plasma thyroid-stimulating hormone (TSH) levels in the normal range are a risk factor for cardiovascular disease and mortality in participants with type 2 diabetes mellitus with high cardiovascular risk.

\section{Methods}

We included 1,265 participants with high cardiovascular risk, type 2 diabetes, and TSH within the normal range $(0.35-5.00 \mathrm{mIU} / \mathrm{L})$ from the Second Manifestations of ARTerial disease cohort. The primary outcome was major cardiovascular events (MACE; vascular death, stroke and myocardial infarction). Secondary outcomes of interest were the separate vascular outcomes and all-cause mortality. Cox proportional hazard models were used to evaluate the risk of plasma TSH levels on all outcomes.

\section{Results}

A total of 191 MACE occurred during a total follow-up of 8,183 years. Plasma TSH levels were not associated with MACE (hazard ratio (HR) per mIU/L TSH increase 0.93; $95 \%$ confidence interval $(95 \% \mathrm{Cl}) 0.80-1.08)$. With a total of 54 strokes during the study period, plasma TSH was associated with a lower risk of stroke (HR per mIU/L 0.64, 95\% Cl 0.45-0.89). There was no association between plasma TSH levels and risk of myocardial infarction, vascular death, or all-cause mortality.

\section{Conclusion}

Higher TSH levels within the normal range are associated with a lower risk of stroke in highrisk patients with type 2 diabetes, but not associated with the risk of other cardiovascular events or mortality. 


\section{Introduction}

Type 2 diabetes is a major risk factor for cardiovascular mortality and morbidity., ${ }^{1,2}$ Despite extensive treatment of classical cardiovascular risk factors in accordance to international guidelines, including blood pressure control and lipid management, a high residual risk for cardiovascular events in people with type 2 diabetes remains. ${ }^{3}$ Characterizing other potential causative factors of this residual risk may lead to new pathophysiological insights and potentially to strategies aimed at further reducing the residual cardiovascular disease (CVD) risk.

It has longer been known that both overt and subclinical hyper- or hypothyroidism are risk factors for incident cardiovascular events and mortality. ${ }^{4-6}$ There is evidence from cross-sectional studies that even in euthyroid subjects, higher levels of plasma TSH are associated with classical cardiovascular risk factors such as low-density lipoprotein (LDL) cholesterol, triglycerides, blood pressure, and adiposity. ${ }^{7-10}$ Additionally, the cardiovascular system is directly influenced by thyroid hormones, with higher triiodothyronine levels leading to relaxation of vascular smooth muscle cells, decreased vascular resistance, increased cardiac contractility, and increased heart rate. ${ }^{11}$ Furthermore, there is an increased prevalence of (subclinical) thyroid dysfunction in people with type 2 diabetes, ${ }^{12-15}$ indicating possible common pathophysiological mechanisms or a possible relationship between thyroid function and type 2 diabetes.

We hypothesized that the cardiovascular effects of type 2 diabetes and the cardiovascular effects of higher thyroid stimulating hormone levels might have a multiplicative effect. We were interested to investigate the relationship between plasma TSH levels in the normal range with the risk for CVD in people with type 2 diabetes, independent of other related traditional risk factors such as cholesterol and blood pressure. Unraveling this association may lead to more understanding of the pathophysiological mechanisms underlying CVD in people with type 2 diabetes that may be a small step in explaining the residual risk for CVD in type 2 diabetes.

The aim of this prospective cohort study was to evaluate the relationship between plasma TSH levels in the normal range and the risk of vascular events and mortality in high-risk patients with type 2 diabetes.

\section{Material and methods}

\section{Study population}

Data were used from the Second Manifestations of ARTerial disease (SMART) study, an ongoing prospective cohort study at the University Medical Center Utrecht, the Netherlands. From September 1996 onwards, patients referred to our institution with 
clinically manifest vascular disease or vascular risk factors were eligible for participation. The study design and rationale of the SMART cohort has been published previously. ${ }^{16}$ To summarize, the participants underwent a standardized vascular screening consisting of a health questionnaire including medical history and cardiovascular risk factors, physical examination and laboratory testing in fasting state. The SMART study complied with the Declaration of Helsinki, ethical approval was obtained from the Medical Ethics Committee of the University Medical Center Utrecht, and written informed consent was obtained from all participants.

For the current study, data were used from 1,372 participants with type 2 diabetes included between July 2003 and March 2017, since TSH was not routinely measured at baseline before July 2003. A formal power calculation has not been performed. Type 2 diabetes was defined as a referral diagnosis of type 2 diabetes, self-reported diagnosis of type 2 diabetes in the questionnaire, the use of glucose-lowering agents or insulin at inclusion, or a glucose plasma concentration of $\geq 7.0 \mathrm{mmol} / \mathrm{L}$ at baseline with commencement of glucose-lowering therapy within 1 year after inclusion. Participants receiving either thyroid hormone supplementation or anti-thyroid medication $(n=46)$ were excluded from analysis. Participants with a baseline TSH measurement $<0.35 \mathrm{mIU} / \mathrm{L}(\mathrm{n}=19)$ or $>5.0 \mathrm{mIU} / \mathrm{L}(\mathrm{n}=42)$ were excluded to restrict the analysis to euthyroid participants $(n=1,265)$, according to the local laboratory reference values (Figure 1). The measurement of TSH is described in more detail in Supplementary Methods 1.

\section{Follow-up}

During follow-up, information on hospitalization, outpatient clinic visits and (cardiovascular) events was obtained biannually through questionnaires. All available data were collected on reported events. Death was reported by the general practitioner, treating specialist, or relatives. All events were independently evaluated by three members of the SMART cohort end point committee.

The primary outcome measure of interest was a composite outcome of major cardiovascular events (MACE; myocardial infarct (MI), stroke, and vascular death). Secondary outcomes of interest were the separate vascular outcomes and all-cause mortality. The definitions of these events have been described previously, ${ }^{16}$ and are included in Supplementary Table 1. Duration of follow-up was defined as the period between study inclusion and development of first cardiovascular follow-up, death, loss to follow-up, or the preselected date of 1 March 2016. In total, 79 (6.2\%) participants were lost to follow-up during the study period. 
Figure 1. Flowchart of selection of study population

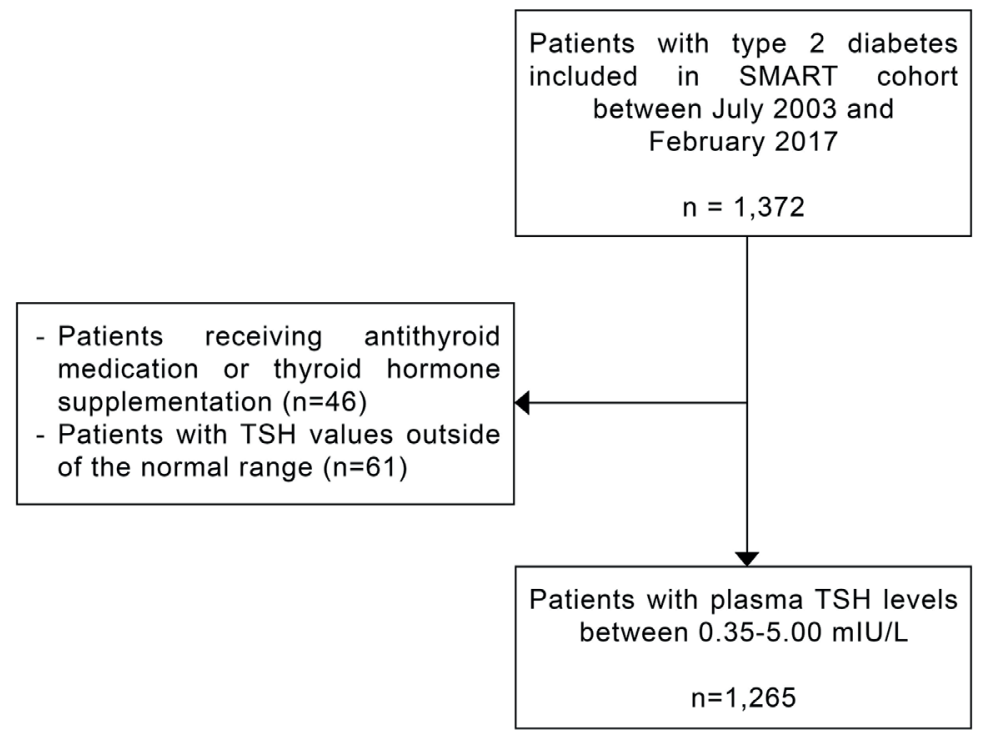

\section{Statistical analysis}

Baseline characteristics are described per quartile of TSH. To prevent overrepresentation of female subjects in the higher quartiles of $\mathrm{TSH}_{1}{ }^{17}$ the data from men and women were ranked separately into quartiles and then combined in sex-pooled quartiles. Normally distributed continuous data are presented as mean \pm standard deviation, whereas unevenly distributed data are presented as median with interquartile range (IQR).

The effect of baseline TSH levels on all new events was evaluated using multiple Cox proportional hazard models, adjusted for potential confounders. The assumption of proportionality was visually checked by plotting Schoenfeld residuals. Linearity of the relation between TSH and risk of the outcome measures was confirmed by restricted cubic splines. The plasma TSH levels were examined as a continuous measure, so that the hazard ratio (HR) with corresponding 95\% confidence intervals $(95 \% \mathrm{Cl}$ ) denotes the increase in risk for the event per one $\mathrm{mIU} / \mathrm{L}$ increase of TSH within the defined normal range of plasma TSH.

In model I, adjustments were made for age and sex only. In model II, additional adjustments were made for possible confounders current smoking, presence of clinically manifest vascular disease at baseline, lipid levels (total cholesterol, HDL cholesterol and triglycerides), renal function as measured by the estimated glomerular filtration rate, calculated using the Chronic Kidney Disease Epidemiology Collaboration (CKDEPI) formula, and blood pressure. As adiposity may be in the causal pathway between 
plasma TSH levels and cardiovascular outcomes, 18,19 it was not included as a confounder in the initial model. Therefore, an exploratory model was created additionally adjusting visceral adipose tissue (VAT) thickness as a measure of adiposity. Another exploratory model was created to assess the impact of preventive cardiovascular therapies, including lipid-lowering, blood-pressure lowering, aspirin, and insulin use, which may influence cardiovascular disease risk, as the study population includes patients with and without clinically manifest vascular disease at baseline. To examine whether the relation between plasma TSH levels and outcomes was modified by adiposity (measured by VAT thickness), or presence of clinically manifest vascular disease at baseline, the interaction between these variables, TSH and risk of the primary outcome was tested. Finally, a sensitivity analysis was performed using reference ranges for plasma TSH levels more commonly encountered in the literature $(0.40-4.12$ and $0.45-4.50 \mathrm{mIU} / \mathrm{L}){ }^{20-22}$

Missing data ( $<1 \%$ of all variables) were imputed by single imputation using predictive mean matching (areglmpute-algorithm in R, Hmisc-package). All analyses were conducted with $\mathrm{R}$ statistical software V.3.5.1 (www.r-project.org, R Foundation for Statistical Computing, Vienna, Austria). For all analyses, a p-value $<0.05$ was considered statistically significant unless stated otherwise.

\section{Results}

\section{Baseline characteristics}

Baseline characteristics of 1,265 participants are shown in Table 1. The mean age of the study population was 61 years (SD 10 years), and $73 \%$ of the participants were male. Their mean BMI was $29 \mathrm{~kg} / \mathrm{m} 2$ (SD $5 \mathrm{~kg} / \mathrm{m} 2$ ) and the mean SBP was $145 \mathrm{mmHg}$ (SD $21 \mathrm{mmHg}$ ). A history of clinically manifest vascular disease was common (69\%), of which a history of coronary artery disease was the biggest group (51\%). $78 \%$ of the study population used lipid lowering medication, and $84 \%$ used blood pressure lowering medication. Across the sex-pooled quartiles, the percentage of (both current and ever) smokers and eGFR decreased, whereas the other baseline characteristics were similar.

\section{Relation between TSH and outcomes}

The median follow-up for all MACE was 6.4 years (IQR 3.3 - 9.6 years), with a total of 191 events (IR 23.3 per 1,000 person-years; 95\% Cl 20.1-26.9). There was no association between plasma TSH levels and the composite outcome of MACE (fully adjusted HR 0.93; $95 \% \mathrm{Cl} 0.80-1.08)$. The risk of stroke $(n=54)$ decreased significantly with higher TSH levels (fully adjusted HR 0.64; $95 \% \mathrm{Cl} 0.45-0.88$ per $1 \mathrm{mIU} / \mathrm{L}$ increase of plasma TSH level, p-value 0.008 ), whereas there was no statistically significant relation with the risk of myocardial 
Table 1. Participant characteristics according to sex-pooled TSH quartiles

\begin{tabular}{|c|c|c|c|c|}
\hline & \multicolumn{4}{|c|}{ Plasma TSH levels in the normal range $(n=1,265)$} \\
\hline & $\begin{array}{l}\text { Quartile 1 } \\
\mathrm{n}=342\end{array}$ & $\begin{array}{l}\text { Quartile 2 } \\
\mathrm{n}=308\end{array}$ & $\begin{array}{l}\text { Quartile } 3 \\
\mathrm{n}=308\end{array}$ & $\begin{array}{l}\text { Quartile 4 } \\
\mathrm{n}=307\end{array}$ \\
\hline TSH range $(\mathrm{mlU} / \mathrm{L})$ & $0.37-1.40$ & $1.23-1.90$ & $1.74-2.60$ & $2.41-5.00$ \\
\hline Male gender & $252(74 \%)$ & $226(73 \%)$ & $226(73 \%)$ & $224(73 \%)$ \\
\hline Age (years) & $61 \pm 10$ & $60 \pm 10$ & $61 \pm 10$ & $62 \pm 09$ \\
\hline Body mass index (kg/m2) & $28 \pm 5$ & $30 \pm 5$ & $30 \pm 5$ & $29 \pm 5$ \\
\hline Waist circumference $(\mathrm{cm})$ & $101 \pm 13$ & $102 \pm 13$ & $103 \pm 14$ & $102 \pm 13$ \\
\hline Systolic blood pressure (mmHg) & $144 \pm 20$ & $144 \pm 20$ & $146 \pm 21$ & $145 \pm 22$ \\
\hline Diastolic blood pressure (mmHg) & $83 \pm 12$ & $83 \pm 12$ & $84 \pm 13$ & $83 \pm 11$ \\
\hline Current smoker & 109 (32\%) & $69(22 \%)$ & $54(18 \%)$ & $43(14 \%)$ \\
\hline Glucose (mmol/L) & $8.3 \pm 2.5$ & $8.0 \pm 2.2$ & $8.2 \pm 2.3$ & $8.1 \pm 2.2$ \\
\hline HbA1c (\%) & $6.9 \pm 1.2$ & $6.8 \pm 1.0$ & $6.9 \pm 1.1$ & $6.9 \pm 1.2$ \\
\hline $\mathrm{HbA1c}(\mathrm{mmol} / \mathrm{mol})$ & $51 \pm 13$ & $50 \pm 10.9$ & $51 \pm 12$ & $51 \pm 13$ \\
\hline Insulin (mIU/L) & $13.0(8.0-21.8)$ & $13.0(8.8-20.0)$ & $14.5(9.9-21.3)$ & $13.0(8.0-20.0)$ \\
\hline Total cholesterol (mmol/l) & $4.4(3.7-5.3)$ & $4.4(3.7-5.2)$ & $4.4(3.8-5.2)$ & $4.4(3.7-5.2)$ \\
\hline HDL-cholesterol (mmol/l) & $1.1(0.9-1.3)$ & $1.1(0.9-1.3)$ & $1.1(1.0-1.3)$ & $1.1(0.9-1.3)$ \\
\hline LDL-cholesterol (mmol/l) & $2.4(1.9-3.0)$ & $2.4(1.9-3.1)$ & $2.4(1.8-3.1)$ & $2.3(1.8-3.0)$ \\
\hline Triglycerides (mmol/l) & $1.5(1.1-2.2)$ & $1.6(1.2-2.4)$ & $1.6(1.1-2.4)$ & $1.7(1.2-2.4)$ \\
\hline eGFR (ml/min/1.73m2) & $79.8 \pm 18.8$ & $78.1 \pm 19.8$ & $76.4 \pm 19.9$ & $73.9 \pm 20.5$ \\
\hline \multicolumn{5}{|l|}{ Medical history } \\
\hline Clinically manifest vascular disease & $255(75 \%)$ & $213(69 \%)$ & 199 (65\%) & 209 (68\%) \\
\hline Coronary artery disease & 197 (58\%) & $153(50 \%)$ & 138 (45\%) & $154(50 \%)$ \\
\hline Cerebrovascular disease & $58(17 \%)$ & $63(20 \%)$ & $56(18 \%)$ & $54(18 \%)$ \\
\hline Peripheral vascular disease & $39(11 \%)$ & $28(9 \%)$ & $28(9 \%)$ & $32(10 \%)$ \\
\hline \multicolumn{5}{|l|}{ Medication use } \\
\hline Oral hypoglycaemic use & $240(70 \%)$ & $218(71 \%)$ & $213(69 \%)$ & $213(69 \%)$ \\
\hline Insulin & $73(21 \%)$ & $69(22 \%)$ & 71 (23\%) & 79 (26\%) \\
\hline Lipid lowering medication & 267 (78\%) & $233(76 \%)$ & 227 (74\%) & 255 (83\%) \\
\hline BP-lowering medication & 282 (82\%) & $263(85 \%)$ & 258 (84\%) & $258(84 \%)$ \\
\hline
\end{tabular}

Abbreviations: $T S H=$ thyroid stimulating hormone; $H D L=$ high-density lipoprotein; $L D L=$ low-density lipoprotein; eGFR = estimated glomerular filtration rate according to Chronic Kidney Disease Epidemiology Collaboration formula.

All data in $n(\%)$, mean \pm standard deviation, or median (interquartile range) 
Figure 2. Relation between plasma TSH levels and occurrence of new vascular events and mortality in participants with type 2 diabetes.

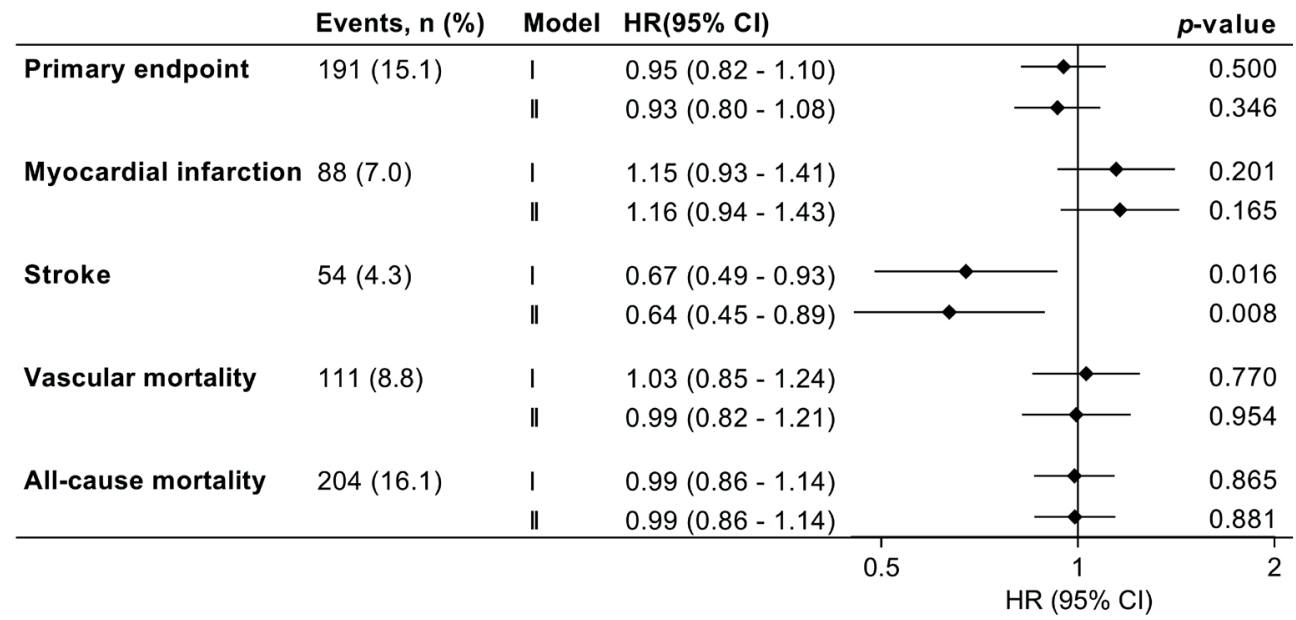

Model 1: Adjusted for age and sex. Model 2: Adjusted for age, sex, current smoking, presence of clinically manifest vascular disease at baseline, estimated glomerular filtration rate, systolic blood pressure, total cholesterol and HDL cholesterol. The hazard ratio denotes the increase in risk for the defined outcome event per one $\mathrm{mIU} / \mathrm{L}$ rise in level of TSH within the normal range (0.35-5.0 $\mathrm{mIU} / \mathrm{L})$.

infarction (HR 1.16; 95\% Cl 0.94-1.43), vascular mortality (HR 0.99; 95\% Cl 0.82-1.21), or all-cause mortality (HR 0.99; $95 \% \mathrm{Cl} 0.86-1.14$ ) (Figure 2). The exploratory models did not change the risk estimates meaningfully.

Adiposity (measured by VAT) or a history of clinically manifest vascular disease did not significantly modify the relation between TSH and the primary outcome MACE ( $p$ for interaction >0.05) and stratification did not change the effect estimates meaningfully. Sensitivity analyses using different reference ranges for plasma TSH levels did not change the risk estimates meaningfully (data not shown).

\section{Discussion}

In this prospective cohort study, higher levels of plasma TSH in the normal range were not related with the risk of the primary outcome of incident MACE in patients with type 2 diabetes. There was a decreased risk of stroke in patients with higher plasma TSH levels within the normal range. There was no association between plasma TSH levels and risk of myocardial infarction, cardiovascular mortality or all-cause mortality. 
The association between TSH levels in the normal range and MACE has previously been examined in several longitudinal cohort studies, but not specifically in people with type 2 diabetes. In studies with differing study domains, there were conflicting results, with one study in elderly patients ( $\geq 65$ years) reporting an association between plasma TSH and a composite outcome including coronary heart disease, heart failure and atrial fibrillation (HR 0.94; 95\% Cl 0.88-1.00). ${ }^{21}$ However, both in the SMART cohort among participants with clinically manifest vascular disease at baseline, and in the Rotterdam Study among 7,785 participants from the general population, plasma TSH levels in the normal range were not associated with MACE. ${ }^{18,23}$ It is possible that, as people with type 2 diabetes already have such a high risk of cardiovascular events and mortality, the hypothesized added effect from thyroid function is unimportant.

The most notable finding in the current study was the association between higher TSH levels within the normal range and a decreased risk of stroke. No previous studies have investigated this association in the domain of people with type 2 diabetes. An individual participant data (IPD) analysis in 34,853 participants from 12 population cohort studies, both with and without diabetes mellitus, found an independent association between higher plasma TSH levels (reference range $0.45-4.50 \mathrm{mIU} / \mathrm{L}$ ) and a decreased risk of stroke (HR per $1 \mathrm{mIU} / \mathrm{L}$ increase $0.78 ; 95 \% \mathrm{Cl} 0.65-0.95) .22$ Thus, considering the overlap of confidence intervals, the association between TSH levels and stroke in people with type 2 diabetes does not seem to be meaningfully different than in the general population.

Of note is that the relationship between TSH and stroke has an opposite direction as we hypothesized. One possible explanation for the inverse effect of plasma TSH levels on stroke, is that higher TSH levels in the normal range give a lower risk of incident atrial fibrillation, ${ }^{24}$ a leading cause of ischemic stroke. ${ }^{25}$ As type 2 diabetes is also associated with atrial fibrillation, this relationship might be especially true for people with type 2 diabetes. Unfortunately, as there was no available information on incident atrial fibrillation in the current study, we could not further investigate this hypothesis. Other possible explanations include a difference in coagulability; both hyperthyroidism and diabetes mellitus are associated with hypercoagulability, 26,27 which is associated with an increased risk of ischemic stroke. ${ }^{28}$ Whether this is also true for low-normal TSH levels is unknown. Finally, differences in TSH levels are associated with classical risk factors for stroke, most notably blood pressure. ${ }^{29,30}$ However, adjustment for blood pressure at baseline did not alter the risk estimates meaningfully, indicating that plasma TSH levels are a risk factor for stroke independent from blood pressure.

In the current study, no associations were found between plasma TSH levels in the normal range and the other secondary outcomes. Studies in differing patient populations have conflicting results, with some studies reporting an association with coronary heart 
disease, ${ }^{18}$ and both cardiovascular or all-cause mortality in some populations, ${ }^{31,32}$ while in other populations no association is found between normal range TSH and hospitalization for myocardial infarction and mortality. ${ }^{23,32-34}$ An IPD analysis in 55,412 individuals from 14 cohorts in the general population showed no association between TSH levels within the reference range (0.45-4.50 mIU/L) and fatal and non-fatal coronary heart disease events. ${ }^{35}$ Additionally, in a Mendelian randomization case-control study in 195,055 participants, no evidence for a causal relation was found between genetically predicted thyroid function (using 34 genetic variants for TSH levels) and ischemic heart disease (odds ratio per SD TSH increase 1.05; 95\% Cl 0.87-1.12). ${ }^{36}$ Theoretically, Mendelian randomization studies are at a low risk of confounding and reverse causality, and thus a method to ascertain causality of observational association. ${ }^{37}$ However, the selected loci only explain part of the total variation in TSH concentration. Furthermore, if genetic variants are actually associated with confounding factors or with the outcome through other pathways than just TSH, it is possible that the found estimate is biased. ${ }^{38}$

Considering the conflicting results from cohort studies, additional IPD analysis or Mendelian randomized studies, both in people with type 2 diabetes and general population, may provide further insights in the causal relation between plasma TSH levels and cardiovascular outcomes and mortality.

These results provide further evidence that TSH concentrations in the range currently considered to be the normal range, are associated with increased risks of stroke, raising the question whether reference ranges based on population distributions are suitable for TSH or should be replaced with reference ranges based on clinical outcomes, and whether treatment aimed at the thyroid function might be of interest for preventive strategies, especially in people with type 2 diabetes.

Major strengths of the present study include the prospective study design, length of follow-up and the availability of data for possible confounders. Medical care was given according to current international guidelines, reflecting current clinical practice. The SMART cohort is representative of people with high cardiovascular risk in Western countries. Furthermore, sensitivity analysis was performed with different reference ranges of plasma TSH, to exclude the possibility that differences between our study and previous studies can be explained by differences in TSH reference ranges.

An important limitation of the study is that plasma TSH was only measured at baseline, and therefore it is not possible to investigate changes of thyroid function over time. Furthermore, we had limited number of participants with an outcome of stroke, and thus limited power. However, the fully adjusted model for the relation between TSH and stroke was highly significant, making it unlikely that this is a chance finding. Additionally, the study population contains both patients with and without clinically manifest vascular disease, 
which may influence the baseline risk of (recurrent) cardiovascular events. However, there is no effect modification by baseline vascular disease status, and adjustment for clinically manifest vascular disease or preventative treatments did not change the estimates meaningfully. Finally, as this is an observational study, residual confounding cannot be excluded.

In conclusion, higher TSH levels within the normal range are associated with a decreased risk of stroke in high-risk patients with type 2 diabetes, but not with the risk of other cardiovascular events or mortality. These findings may help in further understanding pathophysiological mechanisms for the residual risk for CVD in type 2 diabetes. 


\section{References}

1. Ali MK, Bullard KM, Saaddine JB, Cowie CC, Imperatore G, Gregg EW. Achievement of Goals in U.S. Diabetes Care, 1999-2010. N Engl J Med. 2013;368(17):1613-24.

2. Stam-Slob MC, van der Graaf Y, de Borst GJ, Cramer MJ, Kappelle LJ, Westerink J, et al. Effect of Type 2 Diabetes on Recurrent Major Cardiovascular Events for Patients With Symptomatic Vascular Disease at Different Locations. Diabetes Care. 2015;38(8):1528-35.

3. Engelen SE, van der Graaf Y, Stam-Slob MC, Grobbee DE, Cramer MJ, Kappelle LJ, et al. Incidence of cardiovascular events and vascular interventions in patients with type 2 diabetes. Int J Cardiol. 2017;248:301-7.

4. Singh S, Duggal J, Molnar J, Maldonado F, Barsano CP, Arora R. Impact of subclinical thyroid disorders on coronary heart disease, cardiovascular and all-cause mortality: a meta-analysis. Int J Cardiol. 2008;125(1):41-8.

5. Ceresini G, Marina M, Lauretani F, Maggio M, Bandinelli S, Ceda GP, et al. Relationship Between Circulating Thyroid-Stimulating Hormone, Free Thyroxine, and Free Triiodothyronine Concentrations and 9-Year Mortality in Euthyroid Elderly Adults. J Am Geriatr Soc. 2016;64(3):553-60.

6. Chaker L, Baumgartner C, Ikram MA, Dehghan A, Medici M, Visser WE, et al. Subclinical thyroid dysfunction and the risk of stroke: a systematic review and meta-analysis. Eur J Endocrinol. 2014;29(11):791-800.

7. Garduno-Garcia J de J, Alvirde-Garcia U, Lopez-Carrasco G, Padilla Mendoza ME, Mehta R, Arellano-Campos $\mathrm{O}$, et al. TSH and free thyroxine concentrations are associated with differing metabolic markers in euthyroid subjects. Eur J Endocrinol. 2010;163(2):273-8.

8. Zhang Y, Lu P, Zhang L, Xiao X. Association between lipids profile and thyroid parameters in euthyroid diabetic subjects: a cross-sectional study. BMC Endocr Disord. 2015;15:12.

9. Åsvold BO, Bjøro T, Nilsen TIL, Vatten LJ, Asvold BO, Bjoro T, et al. Association between Blood Pressure and Serum Thyroid-Stimulating Hormone Concentration within the Reference Range: A Population-Based Study. J Clin Endocrinol Metab. 2007;92(3):841-5.

10. Westerink J, van der Graaf Y, Faber DR, Visseren FLJ, SMART study group. The relation between thyroid-stimulating hormone and measures of adiposity in patients with manifest vascular disease. Eur J Clin Invest. 2011;41(2):159-66.

11. Danzi S, Klein I. Thyroid disease and the cardiovascular system. Endocrinol Metab Clin North Am. 2014;43(2):517-28.

12. Han C, He X, Xia X, Li Y, Shi X, Shan Z, et al. Subclinical Hypothyroidism and Type 2 Diabetes: A Systematic Review and Meta-Analysis. PLoS One. 2015;10(8):e0135233.

13. Distiller LA, Polakow ES, Joffe BI. Type 2 diabetes mellitus and hypothyroidism: the possible influence of metformin therapy. Diabet Med. 2014;31(2):172-5.

14. Gronich N, Deftereos SN, Lavi I, Persidis AS, Abernethy DR, Rennert G. Hypothyroidism is a Risk Factor for New-Onset Diabetes: A Cohort Study. Diabetes Care. 2015;38(9):1657-64. 
15. Brandt F, Thvilum M, Almind D, Christensen K, Green A, Hegedus L, et al. Morbidity before and after the diagnosis of hyperthyroidism: a nationwide register-based study. PLoS One. 2013;8(6):e66711.

16. Simons PC, Algra A, van de Laak MF, Grobbee DE, van der Graaf Y. Second manifestations of ARTerial disease (SMART) study: rationale and design. Eur J Endocrinol. 1999;15(9):773-81.

17. Hollowell JG, Staehling NW, Flanders WD, Hannon WH, Gunter EW, Spencer CA, et al. Serum TSH, T(4), and thyroid antibodies in the United States population (1988 to 1994): National Health and Nutrition Examination Survey (NHANES III). J Clin Endocrinol Metab. 2002;87(2):489-99.

18. Westerink J, van der Graaf Y, Faber DR, Spiering W, Visseren FLJ, SMART study group. Relation between thyroid-stimulating hormone and the occurrence of cardiovascular events and mortality in patients with manifest vascular diseases. Eur J Prev Cardiol. 2012;19(4):864-73.

19. Fox CS, Pencina MJ, D'Agostino RB, Murabito JM, Seely EW, Pearce EN, et al. Relations of thyroid function to body weight: cross-sectional and longitudinal observations in a community-based sample. Arch Intern Med. 2008;168(6):587-92.

20. Garber JR, Cobin RH, Gharib H, Hennessey J V, Klein I, Mechanick JI, et al. Clinical practice guidelines for hypothyroidism in adults: cosponsored by the American Association of Clinical Endocrinologists and the American Thyroid Association. Thyroid. 2012;22(12):1200-35.

21. Cappola AR, Arnold AM, Wulczyn K, Carlson M, Robbins J, Psaty BM. Thyroid function in the euthyroid range and adverse outcomes in older adults. J Clin Endocrinol Metab. 2015;100(3):1088-96.

22. Chaker L, Baumgartner C, den Elzen WPJ, Collet T-H, Ikram MA, Blum MR, et al. Thyroid Function Within the Reference Range and the Risk of Stroke: An Individual Participant Data Analysis. J Clin Endocrinol Metab. 2016;101(11):4270-82.

23. Bano A, Dhana K, Chaker L, Kavousi M, Ikram MA, Mattace-Raso FUS, et al. Association of Thyroid Function With Life Expectancy With and Without Cardiovascular Disease: The Rotterdam Study. JAMA Intern Med. 2017;

24. Chaker L, Heeringa J, Dehghan A, Medici M, Visser WE, Baumgartner C, et al. Normal Thyroid Function and the Risk of Atrial Fibrillation: the Rotterdam Study. J Clin Endocrinol Metab. 2015;100(10):3718-24.

25. Wolf PA, Abbott RD, Kannel WB. Atrial fibrillation as an independent risk factor for stroke: the Framingham Study. Stroke. 1991;22(8):983-8.

26. Squizzato A, Romualdi E, Buller HR, Gerdes VEA. Clinical review: Thyroid dysfunction and effects on coagulation and fibrinolysis: a systematic review. J Clin Endocrinol Metab. 2007;92(7):241520.

27. Carr ME. Diabetes mellitus: a hypercoagulable state. J Diabetes Complications. 2001;15(1):44-54.

28. Maino A, Rosendaal FR, Algra A, Peyvandi F, Siegerink B. Hypercoagulability Is a Stronger Risk Factor for Ischaemic Stroke than for Myocardial Infarction: A Systematic Review. PLoS One. 2015;10(8):e0133523.

29. Danzi S, Klein I. Thyroid hormone and blood pressure regulation. Curr Hypertens Rep. 
2003;5(6):513-20.

30. Droste DW, Ritter MA, Dittrich R, Heidenreich S, Wichter T, Freund M, et al. Arterial hypertension and ischaemic stroke. Acta Neurol Scand. 2003;107(4):241-51.

31. Inoue K, Tsujimoto T, Saito J, Sugiyama T. Association Between Serum Thyrotropin Levels and Mortality Among Euthyroid Adults in the United States. Thyroid. 2016;26(10):1457-65.

32. Åsvold BO, Bjøro T, Platou C, Vatten LJ. Thyroid function and the risk of coronary heart disease: 12-year follow-up of the HUNT study in Norway. Clin Endocrinol (Oxf). 2012;77(6):911-7.

33. Chaker L, van den Berg ME, Niemeijer MN, Franco OH, Dehghan A, Hofman A, et al. Thyroid Function and Sudden Cardiac Death: A Prospective Population-Based Cohort Study. Circulation. 2016;134(10):713-22.

34. Zhang Y, Chang Y, Ryu S, Cho J, Lee W-Y, Rhee E-J, et al. Thyroid hormones and mortality risk in euthyroid individuals: the Kangbuk Samsung health study. J Clin Endocrinol Metab. 2014;99(7):2467-76.

35. Åsvold BO, Vatten LJ, Bjøro T, Bauer DC, Bremner A, Cappola AR, et al. Thyroid function within the normal range and risk of coronary heart disease: an individual participant data analysis of 14 cohorts. JAMA Intern Med. 2015;175(6):1037-47.

36. Zhao J V, Schooling CM. Thyroid function and ischemic heart disease: a Mendelian randomization study. Sci Rep. 2017;7(1):8515.

37. Lawlor DA, Harbord RM, Sterne JAC, Timpson N, Davey Smith G, Thimpson N, et al. Mendelian randomization: using genes as instruments for making causal inferences in epidemiology. Stat Med. 2008;27(8):1133-63.

38. Burgess $S$, Thompson SG. Avoiding bias from weak instruments in Mendelian randomization studies. Int J Epidemiol. 2011;40(3):755-64. 


\section{Supplementary material}

\section{Supplementary Methods 1. Measurement of thyroid-stimulating hormone (TSH)}

Before November 2006, TSH was quantified using a third-generation assay on a Centaur analyzer (Bayer, Germany). This analyzer had an interassay variation of $0.22 \mathrm{mU} / \mathrm{l}(6 \%), 4.5 \mathrm{mU} / \mathrm{l}(5.5 \%), 16.5 \mathrm{mU} / \mathrm{l}(5.2 \%)$. The functional sensitivity of the Centaur analyzer was $0.02 \mathrm{mU} / \mathrm{l}$ with an interassay imprecision of $20 \%$. Starting December 2006, TSH was measured by a third-generation assay on a DXi analyzer (Beckman Coulter, Woerden, The Netherlands), an analyzer with an interassay variation of 4-8\%, and a functional sensitivity of $0.015 \mathrm{mU} / \mathrm{l}$ with an interassay imprecision of $20 \%$. Correlation between the two analyzers was $r=0.9991$ ( $n=69)$, with an intercept of $-0.05 \mathrm{mU} / \mathrm{l}(95 \% \mathrm{Cl} 0.22$ to 0.12$)$ and a slope of $1.04(95 \% \mathrm{Cl}$ 1.029-1.052) (range 0-95 mU/L). 


\section{Supplementary Table 1. Definitions of study outcomes}

\begin{tabular}{ll}
\hline Outcome & Definition \\
\hline (Non-fatal) Myocardial & At least two of the following: \\
infarction & Chest pain for at least 20 minutes, not disappearing after administration of \\
& nitrates; \\
& ST-elevation $>1 \mathrm{~mm}$ in two following leads or a left bundle branch block on the \\
& ECG; \\
& 3. CK elevation of at least two times the normal value of CK and a MB-fraction \\
& $>5 \%$ of the total CK. \\
& Definite: relevant clinical features which have caused an increase in handicap of \\
at least one grade on the modified Rankin scale, accompanied by a fresh infarct \\
Or a hemorrhage on a repeat CT scan.
\end{tabular}


CHAPTER 4 | Normal-range TSH and CVD in type 2 diabetes 

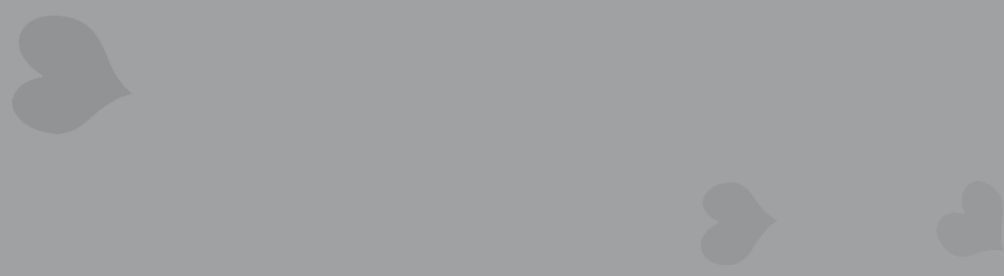

8
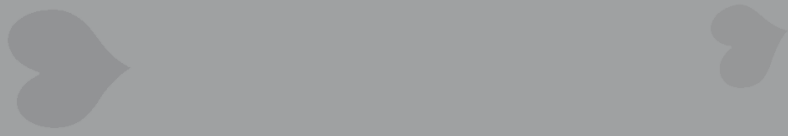

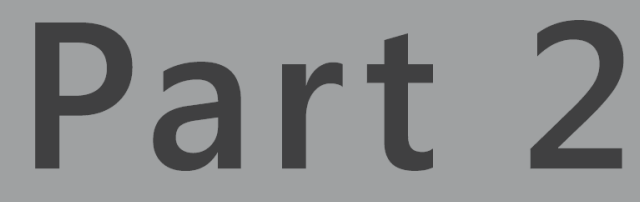

Individualized prediction of treatment effects 


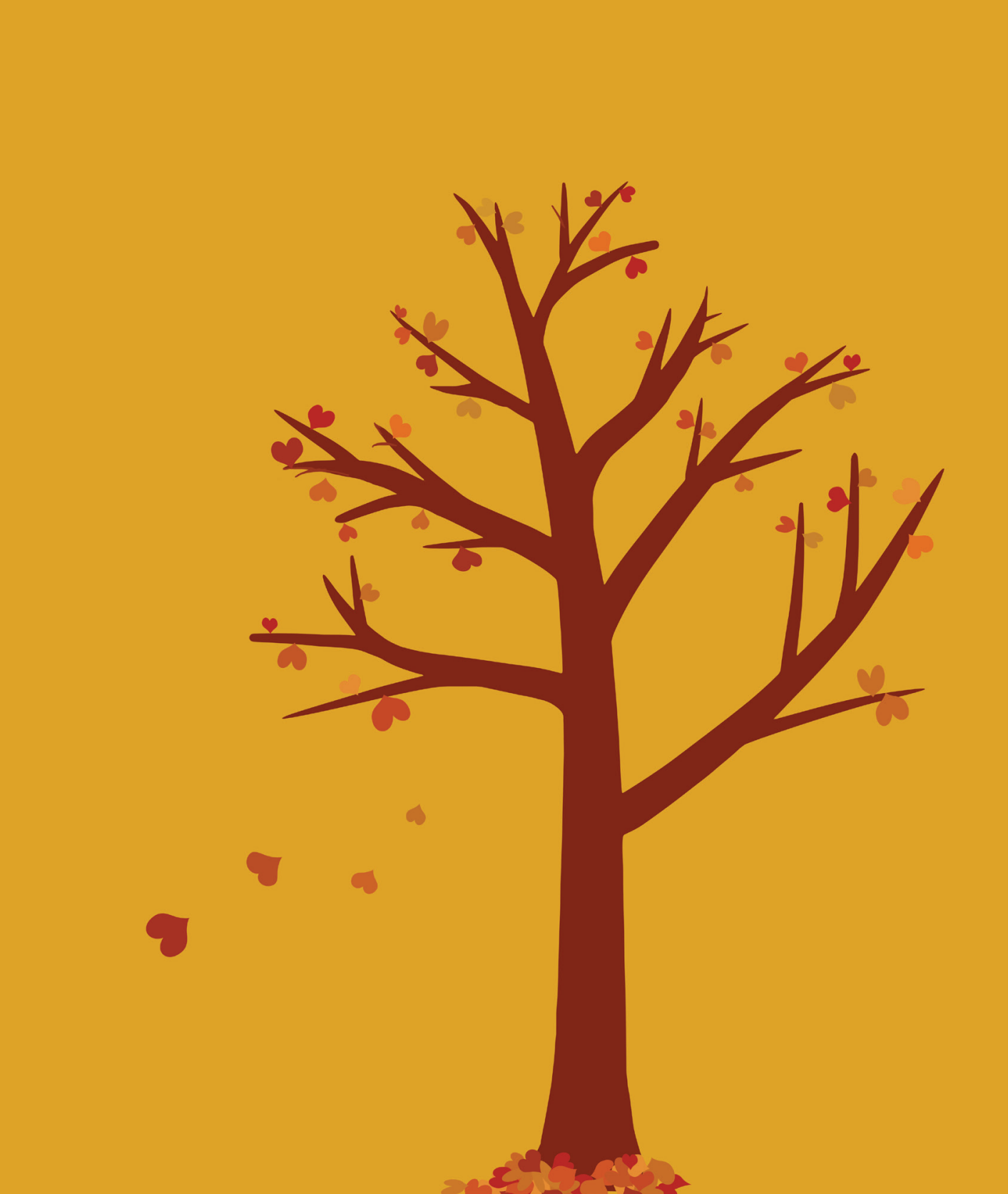


Impact of a patient's baseline risk on the relative benefit and harm of a preventive treatment strategy: applying trial results in

\title{
clinical decision-making
}

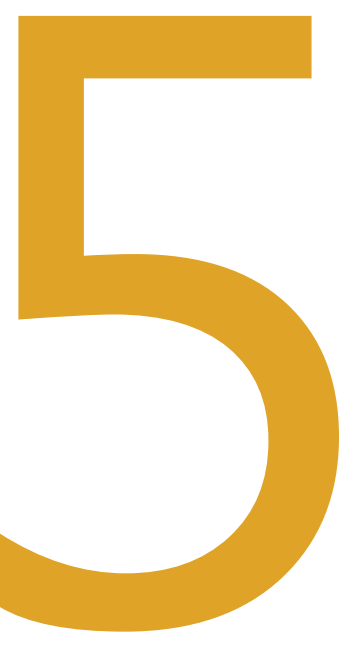

\author{
Tamar I. de Vries \\ Manon C. Stam-Slob \\ Ron J. Peters \\ Yolanda van der Graaf \\ Jan Westerink \\ Frank L.J. Visseren
}

In revision. 


\section{ABSTRACT}

\section{Background}

For translating an overall trial result into an individual patient's expected absolute treatment effect, differences in relative treatment effect between patients need to be taken into account. Traditional one-at-a-time subgroup analyses have major limitations. The aim of the study was to evaluate whether relative treatment effects of medication in two large contemporary trials, is influenced by multivariable baseline risk of an individual patient.

\section{Methods}

In 9,361 patients from the SPRINT trial, risk of the major adverse cardiovascular endpoint (MACE) was assessed using a newly derived risk model. In 18,133 patients from the RELY trial, risk of stroke or systemic embolism (SE) and major bleeding was assessed using the GARFIELD-AF risk model. Heterogeneity of treatment effect was assessed using Cox models of trial allocation, model linear predictor, and their interaction. The models with and without interaction term were compared using the likelihood ratio test. The study population was divided into quartiles based on baseline risk, and quartile-specific hazard ratios for treatment effect were calculated in both trials.

\section{Results}

There was no significant interaction between baseline risk and relative treatment effect from intensive blood pressure lowering on MACE in SPRINT ( $p=0.92)$ or from dabigatran compared to warfarin for stroke or SE in RE-LY $(p=0.71)$. There was significant interaction between baseline risk and treatment effect from dabigatran versus warfarin in RE-LY $(p<0.001)$ on the major bleeding endpoint. Quartile-specific hazard ratio's for bleeding ranged from 0.40 (95\% confidence interval $[\mathrm{Cl}] 0.26-0.61)$ to $1.04(95 \% \mathrm{Cl} 0.83-1.03)$ for dabigatran $110 \mathrm{mg}$ and from $0.61(95 \% \mathrm{Cl} 0.42-0.88)$ to $1.20(95 \% \mathrm{Cl} 0.97-1.50)$ for dabigatran $150 \mathrm{mg}$ compared to warfarin.

\section{Conclusions}

For translation of overall trial results into the expected absolute treatment effects of an individual patient, possible differences in relative treatment effect between patients should be taken into account. Effect modification of the relative treatment effect by individual baseline event risk should be assessed systematically in RCTs using multivariate risk prediction, not only in terms of treatment efficacy but also for important treatment harms, as a pre-specified analysis. 


\section{Introduction}

Every patient is different, and every patient will react differently to medication. However, randomized clinical trials (RCTs) usually report results as a single relative effect size. In current clinical practice, this single relative treatment effect is then used and applied to diverse patient categories and a plethora of individual patients. Implicitly, the assumption is made that this single relative effect measure is true for all study participants, independent of an individual's characteristics.

The anticipated absolute treatment effect is then dependent on not only the relative treatment effect, but also on baseline risk: for example, a relative risk reduction of $20 \%$ for an intervention will result in a $5 \%$ absolute risk reduction (ARR) for a patient with a $25 \%$ baseline 10 -year risk for the outcome, and a $2 \%$ ARR for a patient with a $10 \%$ baseline 10 -year risk. However, for these absolute treatment effects to be true, the assumption of a single relative treatment effect independent of baseline risk and different clinical characteristics needs to hold. An individual patient in clinical practice is not the same as the average trial participant. The dilemma in clinical practice is whether a single overall relative treatment effect is also true for the patient a healthcare professional is seeing. The best treatment on average may not be the best treatment for a given patient. Moreover, a given patient may experience more important treatment harms than another patient.

Simple relative treatment effect modification is regularly assessed in trials using subgroup analyses, a one-characteristic-at-a-time approach. This paper explains why such approaches to subgroup analyses could have limitations, and suggests an alternative method to evaluate relative treatment effect modification. In this paper, we evaluate whether relative treatment effects of medication in two large contemporary trials, are influenced by the baseline risk of an individual patient. This method can be used to assess treatment effect heterogeneity (i.e. how the relative treatment effect varies across patients) in terms of both treatment benefit and treatment harm.

\section{Subgroup analyses: one at a time?}

Subgroup analyses, based on single patient characteristics, are frequently performed to assess differences in the relative treatment effect between groups of patients. However, there are major limitations to subgroup analyses in RCTs. ${ }^{1-4}$ First, stratification and subsequent estimation of relative treatment effects within numerous subgroups results in a high risk of chance findings. ${ }^{1,3}$ Second, one-at-a-time subgroup analyses introduce a "reference class problem". ${ }^{1}$ For example, if both age and sex are effect modifiers, which relative treatment effect measure is the 'correct' one for a young woman? Furthermore, by selecting subgroups on more than one variable at a time, for example by making subgroups based on both age and sex, a low number of endpoints in each subgroup would preclude reliable subgroup analyses. 
A formal statistical interaction test between a patient characteristic of interest and the treatment allocation in a trial is more accurate than the estimation of subgroup-specific treatment effects. However, a true interaction may not be detected as few trials are adequately powered for single variable-treatment interaction analysis. At the same time, if there is no actual interaction effect, the probability of finding a false-positive treatment interaction is $5 \%$ per tested characteristic, of which there are many. Therefore, if effect modification by a single factor can be expected, this should be pre-specified in the trial design and taken into account in trial power estimation.

\section{Assessment of relative treatment effects by baseline risk}

Baseline risk, the risk without the trial intervention, for a clinical outcome can be estimated using a risk model composed of multiple prognostic factors, either based on an existing risk model or derived in the trial itself. Assessment of relative treatment effect modification by individual baseline risk can be used as a method to assess treatment interactions. If no treatment effect modification is expected on the basis of previous data or biological mechanisms, a multivariable approach to relative treatment effect modification has important advantages over one-at-a-time subgroup analyses. First, as this method does not rely on stratification into subgroups, sufficient power may be maintained to assess treatment effect differences in the study population. Second, it is possible that a combination of patient characteristics, rather than a single patient characteristic at a time, influences the treatment effect from an intervention. Third, a single multivariable test for treatment effect modification by baseline risk prevents chance findings due to multiple testing 1 . Furthermore, this approach is based on the data from the trial itself, can be published with the main results from the trial, and if relative treatment effect heterogeneity is apparent, this information facilitates clinical decision making. Finally, relative treatment effect heterogeneity not only pertains to efficacy but also to safety. Therefore, although no clear heterogeneity may be present on treatment efficacy in a trial, there may still be clinical relevant heterogeneity in treatment safety, with important consequences for individualized clinical decision making.

\section{Statistical methodology}

The first step is to estimate baseline risk for individual patients, preferably with an existing risk score. If not all variables are collected in the studies, a new risk score needs to be derived. A time-to-event survival model, e.g. a Cox proportional hazards model, is subsequently fitted with each participant's linear predictor of the risk model using their patient characteristics (which determines an individual's baseline risk) and treatment allocation as predictors. Potential relative treatment effect modification by baseline risk is assessed by adding the interaction term "treatment * linear predictor" to the model. The models with and without interaction term are compared using the likelihood ratio 
test (LRT) with a p-value $<0.05$ indicating a significant interaction. If a statistically significant effect modification is found, the next question is whether this statistical significant difference in relative treatment effect is clinically relevant. By stratifying the study population into quartiles and estimating the relative treatment effect within these quartiles (quartile-specific hazard ratio or relative risk reduction) and comparing these, the presence of relative treatment effect heterogeneity may be assessed. Important to note that the stratification of the study population into quartiles is merely a way to study the change in relative treatment effect with differing baseline risk, and quartile-specific hazard ratios cannot be used in clinical practice.

\section{Methods}

\section{Study populations}

Data were used from the Systolic Blood Pressure Intervention Trial (SPRINT) study and the Randomized Evaluation of Long Term Anticoagulant Therapy (RE-LY) trial.

The SPRINT trial (registration number: NCT01206062) included 9,361 patients aged 50 or older with systolic blood pressure (SBP) of $130 \mathrm{mmHg}$ or higher and an increased risk for cardiovascular disease. Eligible participants were randomized 1:1 to an intensive target SBP of less than $120 \mathrm{mmHg}$, or a standard target SBP of less than $140 \mathrm{mmHg}$. The study was ended prematurely on the basis of a reduced risk in the primary composite major adverse cardiovascular endpoint (MACE) at interim analysis. The RE-LY trial (registration number: NCT00262600) included 18,133 patients with atrial fibrillation, and randomized them 1:1:1 to warfarin, dabigatran $110 \mathrm{mg}$ twice daily, or dabigatran $150 \mathrm{mg}$ twice daily. Most participants had an indication for oral anticoagulation therapy based on their CHA2DS2-VASc score. The randomized controlled trial demonstrated that in patients with atrial fibrillation, dabigatran given at a dose of $110 \mathrm{mg}$ twice daily was associated with a similar risk of stroke and systemic embolism compared to warfarin, while having lower rates of major bleeding. Dabigatran at a dose of $150 \mathrm{mg}$ twice daily was associated with lower rates of stroke and systemic embolism and similar rates of major bleeding compared to warfarin. Detailed descriptions of both trials and inclusion and exclusion criteria have been published previously. ${ }^{5-8}$ (Ethical) approval was obtained from the national regulatory authorities and ethical committees of the participating centers, and all participants provided written informed consent.

\section{Statistical methodology}

Individual treatment effect estimation of intensive blood pressure lowering Since existing risk scores like the Framingham risk score, SCORE risk chart, or ASCVD risk score could not be used for risk estimation due to missing variables and different 
definitions of the primary outcome, ${ }^{9-11}$ a prediction model for the primary composite endpoint (myocardial infarction, other acute coronary syndromes, stroke, heart failure, or death from cardiovascular causes) was derived in the control arm of the SPRINT study using Cox proportional hazards analysis. Well-known predictors were selected from previously published risk scores. ${ }^{9-13}$ Details on model development and model validation for estimation of the risk of the primary endpoint are presented in the Supplementary Methods and Supplemental Figure 1. First, baseline mortality risk was estimated by entering individual patient characteristics in the model formula. Using the methodology as described above, heterogeneity of treatment effect was then assessed using the linear predictor from the newly derived risk model.

Individual treatment effect estimation of dabigatran on stroke and major bleeding in atrial fibrillation

Baseline 1-year risk for the primary endpoint stroke and systemic embolism (SE) and for the risk of major bleeding were estimated using the externally validated Global Anticoagulant Registry in the FIELD-Atrial Fibrillation (GARFIELD-AF) risk models. Details on the model derivation and endpoint definitions have been published previously. ${ }^{14}$ The models were based on the following predictors: age, history of vascular disease, history of stroke, history of bleeding, history of heart failure, chronic kidney disease, region, ethnicity, and oral anticoagulant use. Model performance was assessed with the c-statistic (95\% confidence interval [CI]) for discrimination and with calibration plots of predicted versus observed risk (Supplemental Figure 2). First, baseline risk of stroke/SE and major bleeding were estimated by filling in individual patient characteristics in the GARFIELD-AF model formulas. Using the methodology described above, heterogeneity of treatment effect was then assessed using the linear predictors of respectively the stroke/SE and major bleeding GARFIELD-AF risk functions.

\section{Results}

\section{Baseline characteristics and events}

Baseline characteristics of both trials are shown in Table 1. During a median follow-up of 3.2 years (interquartile range [IQR] 2.7-3.8) in the SPRINT trial, 319 endpoints occurred (6.8\%) in those who received standard treatment, compared to 243 endpoints (5.2\%) in patients who received intensive treatment. During a median follow-up of 2.0 years in RELY (IQR 1.6-2.4), 160 primary events (2.7\%) and 426 major hemorrhages (7.1\%) occurred in participants who received warfarin, compared to 295 primary events (2.4\%) and 757 major hemorrhages (6.3\%) in patients who received dabigatran in either dose. 
Table 1. Baseline characteristics of the SPRINT and RE-LY study populations

\begin{tabular}{lll}
\hline & \multicolumn{1}{l}{ SPRINT } & RE-LY \\
& $\mathrm{n}=9361$ & $\mathrm{n}=18113$ \\
\cline { 2 - 3 } Age (years) & $6029 \pm 64$ & $71 \pm 9$ \\
Male sex & $5399 \pm 58$ & $11514 \pm 64$ \\
Ethnicity & & \\
Caucasian & $5399(58 \%)$ & $12616(70 \%)$ \\
Black & $2802(30 \%)$ & $176(1 \%)$ \\
Hispanic & $984(11 \%)$ & $879(5 \%)$ \\
Other & $176(2 \%)$ & $4442(24 \%)$ \\
Current smoking & $1244(13 \%)$ & $5979(33 \%)$ \\
History of cardiovascular disease & $1562(17 \%)$ & $5248(29 \%)$ \\
Systolic blood pressure (mmHg) & $140 \pm 16$ & $131 \pm 18$ \\
Body mass index (kg/m2) & $30 \pm 6$ & $29 \pm 6$ \\
Total cholesterol (mmol/l) & $4.9 \pm 1.1$ & $4.7 \pm 1.1$ \\
LDL choelsterol (mmol/l) & $2.9 \pm 0.9$ & $\mathrm{~N} / \mathrm{A}$ \\
HDL cholesterol (mmol/l) & $1.4 \pm 0.4$ & $\mathrm{~N} / \mathrm{A}$ \\
Estimated glomerular filtration rate (ml/min/1.73m2) & $67 \pm 24$ & $73 \pm 28$ \\
Uses a statin & $4083(44 \%)$ & $8057(45 \%)$ \\
Uses antihypertensives & $8479(91 \%)$ & $14509(80 \%)$ \\
\hline N/A = not avalla. All & 50 & \\
\hline
\end{tabular}

$N / A=$ not available. All data are shown as $n(\%)$ or mean $\pm S D$

\section{Baseline risk and treatment effect heterogeneity}

In the SPRINT trial, estimated absolute 3.2-year risk for the primary endpoint with standard treatment (i.e. baseline risk) varied widely, from $1 \%$ to $50 \%$ absolute risk (Figure 1c). There was no significant interaction between baseline risk for the primary endpoint and the relative treatment effect of intensive treatment in the trial ( $p$-value for interaction $=0.92$ ). The baseline risk quartile-specific hazard ratios are shown in Figure 2.

In RE-LY, estimated baseline absolute 1-year risk of stroke or SE, estimated with the GARFIELD-AF risk model, varied from $0.1 \%$ to $23.5 \%$ while the 1 -year risk of major bleeding varied from $0.3 \%$ to $13.9 \%$ (Figure $1 \mathrm{a}$ and $1 \mathrm{~b}$, respectively). There was no significant interaction between the baseline risk for the primary efficacy endpoint and relative treatment effect of either dabigatran $110 \mathrm{mg}$ or dabigatran $150 \mathrm{mg}$ compared to warfarin (LRT $p$-value for interaction 0.71). There was however a significant interaction between baseline risk of major bleeding and the relative treatment effect of both dabigatran $110 \mathrm{mg}$ 
Figure 1. Distribution of untreated (i.e. baseline) risk of (A) stroke/SE, and (B) major bleeding in the RE-LY trial, and (C) of the primary outcome in the SPRINT trial.
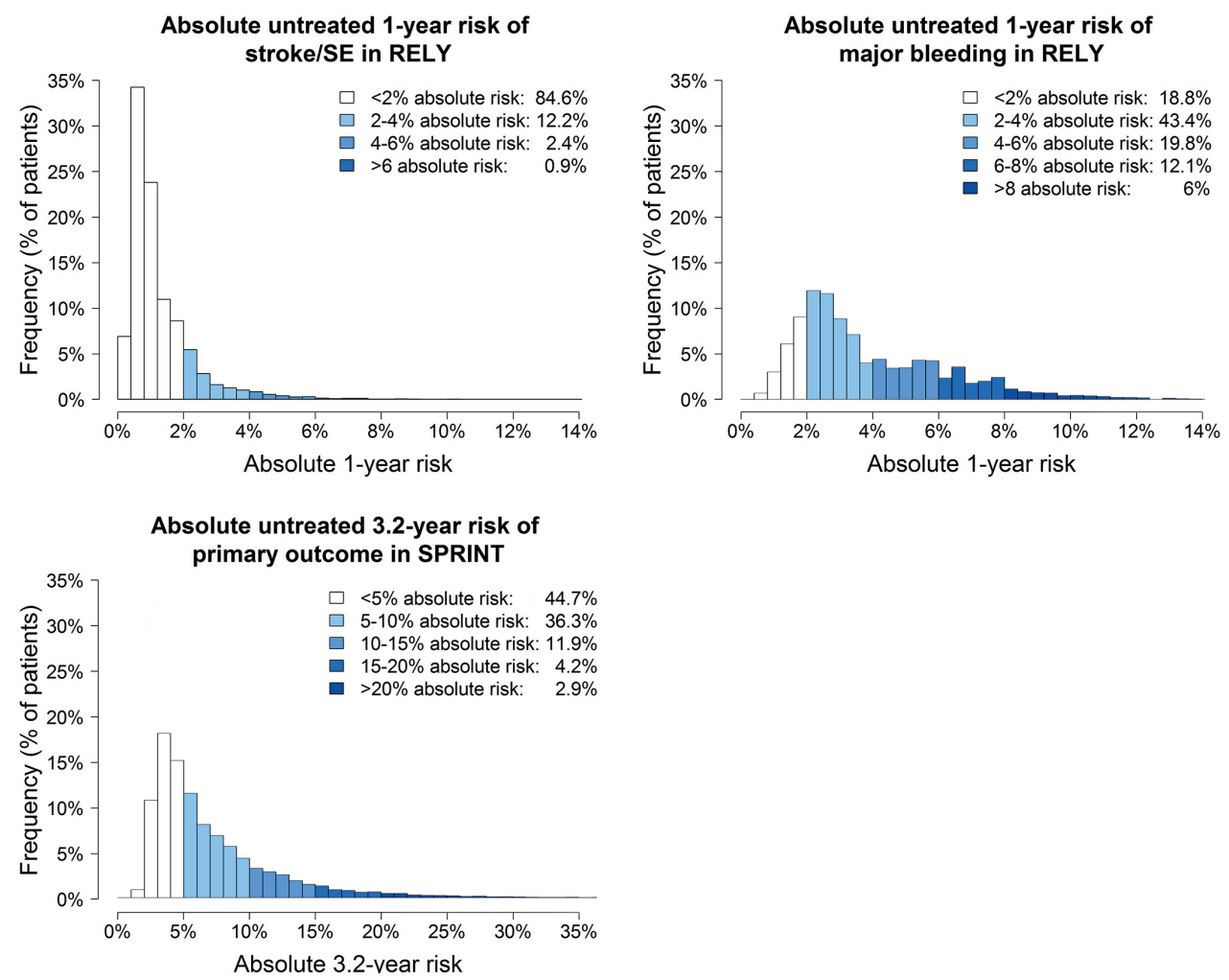

and $150 \mathrm{mg}$ compared to warfarin (LRT $p$-value for interaction <0.001). The baseline risk quartile-specific hazard ratios for the primary endpoint and major bleeding are presented in Figure 3a and 3b, respectively. Quartile-specific hazard ratio's ranged from 0.40 (95\% confidence interval [Cl] $0.26-0.61)$ to $1.04(95 \% \mathrm{Cl} 0.83-1.03)$ for dabigatran $110 \mathrm{mg}$ and from $0.61(95 \% \mathrm{Cl} 0.42-0.88)$ to $1.20(95 \% \mathrm{Cl} 0.97-1.50)$ for dabigatran $150 \mathrm{mg}$ compared to warfarin. Baseline characteristics for the quartiles of estimated baseline risk of major bleeding, and the quartile-specific hazard ratios for the primary endpoint according to major bleeding quartiles are shown in Supplementary Table 1 and Supplementary Figure 3 , respectively. 
Figure 2. Relative treatment effect in the SPRINT study of intensive versus standard blood pressure control in quartiles of baseline risk for the primary endpoint.

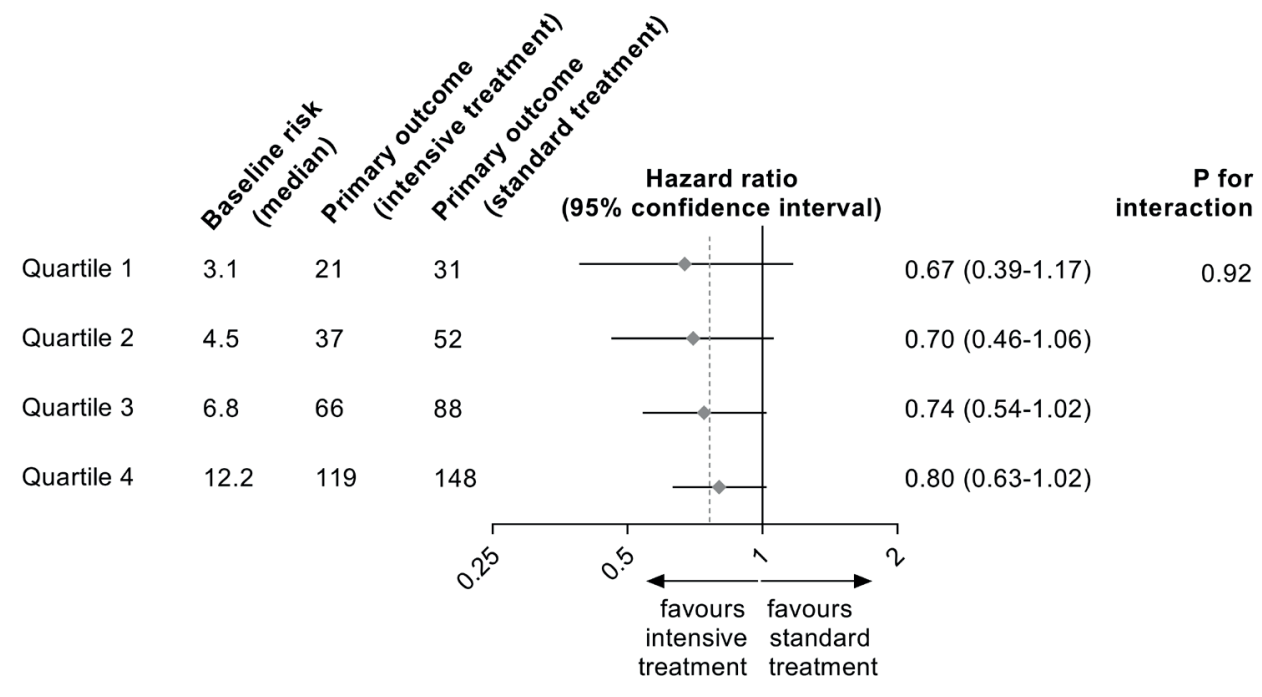

\section{Discussion}

Conventionally, an individual patient's expected absolute treatment effect is calculated from their baseline risk and the average relative treatment effect as observed in a clinical trial. However, the relative treatment effect may not be uniform across the trial population. The relative treatment effect may be dependent on an individual's baseline event risk. In the current study, relative treatment effect heterogeneity by an individual's baseline risk was assessed in two large randomized clinical trials. In the SPRINT study, there was no evidence for effect modification of the treatment effect from intensive versus standard blood pressure control on basis of baseline MACE risk estimated using a newly derived risk model. In the RE-LY trial, no effect modification for the treatment effect of dabigatran versus warfarin by baseline risk based on the GARFIELD-AF risk model was observed for the risk of the primary endpoint of stroke and systolic embolism. However, we did establish effect modification by baseline bleeding risk on the treatment effect for the risk of harm from major bleeding, with the lowest baseline bleeding risk quartiles having a clear benefit from dabigatran for both doses, while in the highest bleeding risk quartiles there is no lower bleeding risk from dabigatran compared to warfarin.

The clinical consequence of this finding is that the average trial result from the SPRINT study can be applied in all patients. Based on the results of the RE-LY trial, the conclusion is that a single relative treatment effect can be used in all patients for the effect of treatment on the primary efficacy endpoint, but the single relative effect on major bleeding may not be used in all patients. In individualized clinical decision making, from 
Figure 3. Relative effect in RE-LY of dabigatran versus warfarin on the risk of (a) major cardiovascular events in quartiles of baseline risk of major cardiovascular events according to the GARFIELD-AF risk model, and (b) major bleeding in quartiles of baseline risk of major bleeding according to the GARFIELD-AF risk model.

(A)

Dabigatran $110 \mathrm{mg}$

Quartile $1 \quad 0.4 \quad 23$

Quartile $2 \quad 0.8 \quad 31$

Quartile $3 \quad 1.1 \quad 54$

Quartile $4 \quad 2.2 \quad 63$

Dabigatran $150 \mathrm{mg}$

Quartile $1 \quad 0.4 \quad 16 \quad 18$

$\begin{array}{llll}\text { Quartile } 2 & 0.8 \quad 23 & 31\end{array}$

$\begin{array}{llll}\text { Quartile } 3 \quad 1.1 & 38 & 42\end{array}$

Quartile $4 \quad 2.2 \quad 47$

18

31

42

69

18

69
(B)

Dabigatran $110 \mathrm{mg}$

Quartile 1

Quartile $2 \quad 2.7 \quad 62$

Quartile $3 \quad 4.0 \quad 10$

Quartile $4 \quad 6.7 \quad 154$

Dabigatran 150mg

$\begin{array}{llll}\text { Quartile } 1 & 1.9 & 46\end{array}$

$\begin{array}{llll}\text { Quartile 2 } & 2.7 & 81 & 94\end{array}$

$\begin{array}{llll}\text { Quartile } 3 \quad 4.0 \quad 100 & 114\end{array}$

$\begin{array}{lll}\text { Quartile } 4 & 6.7 & 183\end{array}$
Hazard ratio

(95\% confidence interval)
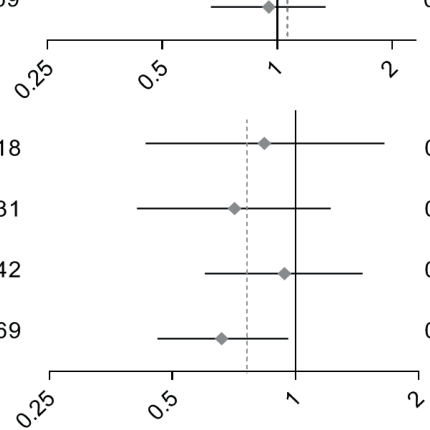

$0.84(0.43-1.65)$

$0.71(0.41-1.22)$

$0.94(0.60-1.46)$

$0.66(0.46-0.96)$
$.25(0.67-2.31)$
$P$ for

interaction

( $95 \%$ confidence interval)

$0.40(0.26-0.61)$

$<0.001$

$0.64(0.47-0.89)$

$0.90(0.69-1.18)$

$1.04(0.83-1.30)$

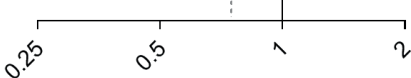

73
94
114
145

$0.61(0.42-0.88)$

0.002

$0.87(0.64-1.17)$

$0.90(0.68-1.17)$

$1.20(0.97-1.50)$ 
the efficacy standpoint, this may entail offering treatment to all patients, as all will benefit in accordance with their individual baseline risk in combination with the overall hazard ratio from the trial (absolute risk reduction). From the safety standpoint, however, when stratifying the study population in quartiles based on their baseline major bleeding risk (i.e. the risk when treating with warfarin) there is a clinically important difference in the relative treatment effect in these quartiles. For dabigatran at a dose of $110 \mathrm{mg}$, a benefit of dabigatran over warfarin is observed in the lowest quartile of predicted bleeding risk, but not in the highest. For dabigatran at a dose of $150 \mathrm{mg}$, a benefit of dabigatran compared to warfarin as observed in the lowest two quartiles of risk is offset by a numerical, though just not statistically significant, detrimental effect in the quartile with the highest baseline bleeding risk. This may explain the neutral average main effect on major bleeding reported in the trial (HR 0.93; 95\% $\mathrm{Cl} 0.81-1.07) .{ }^{6}$ The example shows a potential pitfall in the assessment of heterogeneity of treatment effect; looking at efficacy of a treatment alone potentially gives incomplete information necessary for clinical decision making. Assessment of heterogeneity of treatment effects may be most useful if a treatment strategy is costly or confers harms, as physicians may consider to withhold such treatment from patients with low expected benefit or important expected harm. Anticoagulation, with a risk of major bleeding, represents an example where this approach is clinically useful. For all therapies potential treatment benefits should be weighed against potential harms from treatment. For example, a trial investigating strict blood pressure lowering in frail individuals may warrant investigation of treatment heterogeneity on possible adverse effects such as falls and cognitive decline.

When effect modification is present, further analysis of the data and literature is necessary. In the case of the RE-LY trial, effect modification by both renal function and age has been described in univariate subgroup analyses. ${ }^{15,16}$ However, as discussed before, univariate subgroup analyses have disadvantages including a limited power with a risk of false positive subgroup finding. Our multivariable approach also identified renal function and age as important factors. A reassuring finding is that interaction analysis between both age and renal function, and treatment effect remain statistically significant in an exploratory analysis adjusting for potential confounders (including sex, SBP, history of CVD, and smoking status; data not shown), making it unlikely that these findings are false positive. The advantage of a multivariable risk-based approach, as described in the current study, includes that the aforementioned "reference class problem" is avoided. For example, an 80 year old patient has an increased risk for extracranial major bleeding with either doses of dabigatran compared to warfarin according to univariate subgroup analyses. ${ }^{16}$ At the same time an individual with a glomerular filtration rate $\geq 80 \mathrm{ml} / \mathrm{min}$ has a remarkably decreased risk of major bleeding, while there is a similar risk of bleeding between dabigatran and warfarin in patients with an eGFR $<50 \mathrm{ml} / \mathrm{min}^{15}$ Using just univariate subgroup analyses, 
it may be difficult to decide whether age or renal function is the more important factor influencing treatment response. A multivariable risk-based approach is therefore more appropriate. This can lead to individual absolute treatment effects in terms of both benefit and harm, which can be weighed in clinical practice to make treatment decisions.

When heterogeneity in treatment effect is found, potentially no single characteristic may be identified that drives treatment effect modification. In that situation a "one treatment fits all" approach does not apply and simple subgroup analyses do not solve the problem. A risk model can then be used in clinical practice to determine whether an individual patient qualifies for therapy. For example, patients with atrial fibrillation with a very low risk of major bleeding will likely be better off with dabigatran $150 \mathrm{mg}$, as they will have a lower risk of major bleeding with dabigatran than with warfarin. At the same time, patients with a higher risk of major bleeding will likely be better off with dabigatran $110 \mathrm{mg}$ or even warfarin, as they may have an increased risk of major bleeding with dabigatran $150 \mathrm{mg}$ that offsets the reduction in the risk of stroke/SE that is the uniform across all patients.

The advantage of using a pre-existing, externally validated risk model, such as GARFIELD$A F$, as compared to a newly derived risk model, is that it can be used in clinical practice for reliable, unbiased estimates of baseline risk. Furthermore, if relative treatment heterogeneity is present, an existing risk score is likely easier to implement in clinical practice to calculate individual absolute treatment effects for medical decision making. If possible, when designing a new trial, an existing risk model should be selected, so that the appropriate determinants can be collected at baseline. If no risk models exist for the prediction of the primary endpoint risk, it may be necessary to include the derivation of a new risk model in the trial design if a sufficiently large number of events for model development is anticipated. To deal with the short-comings of traditional subgroup analyses, we propose that assessment of relative treatment heterogeneity using a multivariable approach should be a pre-specified analysis for RCTs.

There are several additional points to further consider when assessing relative treatment effect heterogeneity. First, when a relative treatment effect is independent of baseline risk - and thus, the same relative effect applies to all patients - the variation in absolute treatment effect may still be large if the range of baseline risk is large. ${ }^{17}$ On the other hand, if there is relative treatment heterogeneity, with a significant $p$-value for interaction, this does not automatically imply an important treatment heterogeneity. Thus, both the assessment of relative treatment heterogeneity, and the estimation of individual absolute treatment effects are of critical importance for the translation of clinical trial results to all patients in clinical practice.

There are limitations to the current methodology to consider. In the example of the SPRINT trial, an internally derived risk model was used. External validation of this model should be performed before it is applied in clinical practice. However, for the assessment 
of a risk-treatment interaction, an internally developed model is appropriate, when the internal model performance is sufficient. ${ }^{18}$ Thus, the presented model renders unbiased estimates of treatment effect across the spectrum of baseline risk. Furthermore, a possible limitation of the current methodology is that in trials with strict participant selection criteria, the heterogeneity in patient characteristics may be too small to detect treatment effect modification by baseline risk, and, additionally, the question is whether a risk model derived in a trial population can be generalized to the general population. A careful evaluation of the representativeness of study results is essential for the application of trial results to individuals in clinical practice.

\section{Conclusion}

In conclusion, for translation of an overall trial result into an individual's expected absolute treatment effect, possible differences in relative treatment effect between patients should be taken into account. Effect modification of the relative treatment effect by individual baseline event risk should be assessed systematically in RCTs using multivariable risk prediction, not only in terms of treatment efficacy but also for important treatment harms, as a pre-specified analysis. Relative treatment effects can then be translated more reliably to individual absolute treatment effects which can be weighed for individualized clinical decision making. By using individual patient data in a trial, relative treatment effect modification can be assessed across the full spectrum of risk. 


\section{References}

1. Kent DM, Steyerberg E, van Klaveren D. Personalized evidence based medicine: predictive approaches to heterogeneous treatment effects. BMJ. 2018;363:k4245.

2. Parker $A B$, Yusuf $S$, Naylor $C D$. The relevance of subgroup-specific treatment effects: the Studies Of Left Ventricular Dysfunction (SOLVD) revisited. Am Heart J. 2002;144(6):941-7.

3. Rothwell PM. Treating individuals 2. Subgroup analysis in randomised controlled trials: importance, indications, and interpretation. Lancet. 2005;365(9454):176-86.

4. Yusuf S, Wittes J, Probstfield J, Tyroler HA. Analysis and interpretation of treatment effects in subgroups of patients in randomized clinical trials. JAMA. 1991;266(1):93-8.

5. Ezekowitz MD, Connolly S, Parekh A, Reilly PA, Varrone J, Wang S, et al. Rationale and design of RE-LY: randomized evaluation of long-term anticoagulant therapy, warfarin, compared with dabigatran. Am Heart J. 2009 May;157(5):805-10, 810.e1-2.

6. Connolly SJ, Ezekowitz MD, Yusuf S, Eikelboom J, Oldgren J, Parekh A, et al. Dabigatran versus Warfarin in Patients with Atrial Fibrillation. N Engl J Med. 2009 Sep 17;361(12):1139-51.

7. Ambrosius WT, Sink KM, Foy CG, Berlowitz DR, Cheung AK, Cushman WC, et al. The design and rationale of a multicenter clinical trial comparing two strategies for control of systolic blood pressure: the Systolic Blood Pressure Intervention Trial (SPRINT). Clin Trials. 2014;11(5):532-46.

8. SPRINT Research Group, Wright J, Williamson J, Whelton P, Snyger J, Sink K, et al. A Randomized Trial of Intensive versus Standard Blood-Pressure Control. N Engl J Med. 2015;373(22):2103-16.

9. Wilson PW, D'Agostino RB, Levy $D$, Belanger AM, Silbershatz $H$, Kannel WB. Prediction of coronary heart disease using risk factor categories. Circulation. 1998 May;97(18):1837-47.

10. Conroy RM, Group on behalf of the S project, Pyörälä K, Group on behalf of the S project, Fitzgerald AP, Group on behalf of the $S$ project, et al. Estimation of ten-year risk of fatal cardiovascular disease in Europe: the SCORE project. Eur Heart J. 2003 Jun 1;24(11):987-1003.

11. Goff DCJ, Lloyd-Jones DM, Bennett G, Coady S, D'Agostino RB, Gibbons R, et al. 2013 ACC/ AHA Guideline on the Assessment of Cardiovascular Risk: A Report of the American College of Cardiology/American Heart Association Task Force on Practice Guidelines. J Am Coll Cardiol. 2014;63(25):S49-73.

12. Kaasenbrood L, Bhatt DL, Dorresteijn JA, Wilson PW, D'Agostino RB, Massaron JM, et al. Estimated life-expectancy without recurrent cardiovascular events in patients with vascular disease: the REACH-SMART model. J Am Hear. 2018;7:e009217.

13. Jaspers NEM, Ridker PM, Dorresteijn JAN, Visseren FLJ. The prediction of therapy-benefit for individual cardiovascular disease prevention: rationale, implications, and implementation. Curr Opin Lipidol. 2018;29(6):436-44.

14. Fox KAA, Lucas JE, Pieper KS, Bassand J-P, Camm AJ, Fitzmaurice DA, et al. Improved risk stratification of patients with atrial fibrillation: an integrated GARFIELD-AF tool for the prediction of mortality, stroke and bleed in patients with and without anticoagulation. BMJ Open. 2017 Dec 1;7(12):e017157. 
15. Hijazi Z, Hohnloser SH, Oldgren J, Andersson U, Connolly SJ, Eikelboom JW, et al. Efficacy and safety of dabigatran compared with warfarin in relation to baseline renal function in patients with atrial fibrillation: a RE-LY (Randomized Evaluation of Long-term Anticoagulation Therapy) trial analysis. Circulation. 2014;129(9):961-70.

16. Lauw MN, Eikelboom JW, Coppens M, Wallentin L, Yusuf S, Ezekowitz M, et al. Effects of dabigatran according to age in atrial fibrillation. Heart. 2017 Jul 1;103(13):1015 LP - 1023.

17. Kent DM, Nelson J, Dahabreh IJ, Rothwell PM, Altman DG, Hayward RA. Risk and treatment effect heterogeneity: re-analysis of individual participant data from 32 large clinical trials. Int J Epidemiol. 2016;45(6):2075-88.

18. Burke JF, Hayward RA, Nelson JP, Kent DM. Using internally developed risk models to assess heterogeneity in treatment effects in clinical trials. Circ Cardiovasc Qual Outcomes. 2014;7(1):163-9. 


\section{Supplementary material}

\section{Supplementary Methods 1. Model derivation and estimation of baseline risk in the SPRINT trial}

A prediction model was derived for the combined outcome (myocardial infarction, other acute coronary syndromes, stroke, heart failure, or death from cardiovascular causes) in 9,361 patients from the SPRINT study population. Prespecified predictors selected on basis of previous risk models and availability in the study data were: age, sex, current smoking, African-American race, history of cardiovascular disease, total cholesterol, HDL cholesterol, estimate glomerular filtration rate (using the CKD-EPI formula), and the use of antihypertensive medication at baseline.1-5 Baseline missing variables were singly imputed using predictive mean matching (areglmpute-algorithm in Rstudio, Hmisc-package). Continuous predictors were truncated at the 1st and 99th percentile to limit the effect of outliers. Whether the association of continuous predictors with the outcome variable is log-linear was assessed with restricted cubic splines; to improve the robustness of the model, transformation was applied when this improved model fit, based on Akaike's Information Criterion. 6 Model coefficients of the final model were uniformly shrunken to account for over-optimism with a factor of $4.0 \%$ derived from model selection in 1000 bootstrap samples based on the AIC. The model was fitted for the prediction of 3.2-year risk (median follow-up). Model performance was assessed with the c-statistic (95\% confidence interval $[\mathrm{Cl}]$ ) for discrimination using 1000 bootstrap samples and with calibration plots of predicted versus observed risk.

This is the underlying formula for the prediction of the risk of the primary outcome:

3.2-year risk: $1-0.941 \wedge \exp (\mathbf{L P}+9.416) * 100 \%$

$\mathbf{L P}=-1.382$ * (age in years $/ 10)+0.137$ * ((age in years $\left./ 10)^{2}\right)-0.234$ * (if male) +0.549 (if current smoker) $-0.092 *(\mathrm{SBP}$ in $\mathrm{mmHg})+0.0003 *(\mathrm{SBP} \text { in } \mathrm{mmHg})^{2}-0.501$ (if history of cardiovascular disease) 0.700 * (HDL-c in mmol/L) + 0.174 * (total cholesterol in mmol/L) -0.319 * (eGFR in $\mathrm{ml} / \mathrm{min}$ ) + 0.062 (if African-American) +0.207 (if currently using antihypertensives) $+0.479 *$ ((age in years $/ 10)$ if history of cardiovascular disease) -0.044 * ((age in years $/ 10)^{2}$ if history of cardiovascular disease) 
Supplementary Figure 1. Internal validation of the risk model derived in the SPRINT study

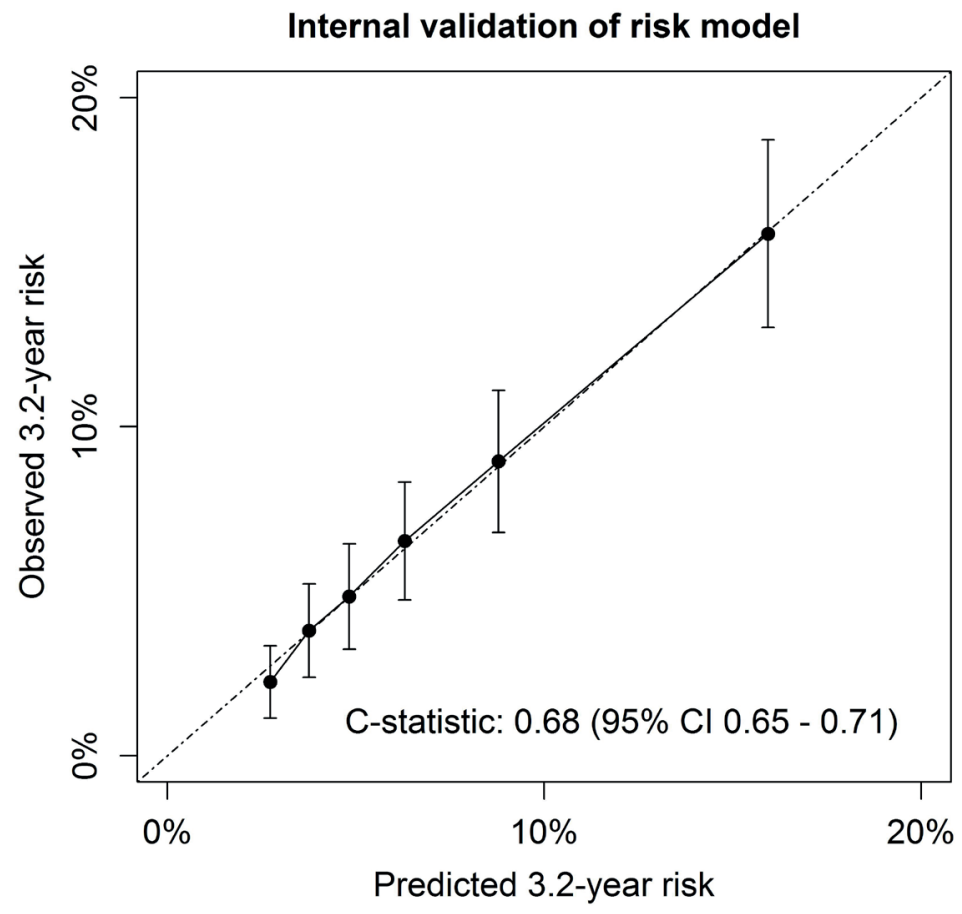


Supplementary Figure 2. External validation of the GARFIELD-AF risk model for (A) stroke and systemic embolism, and (b) major bleeding risk in the RE-LY trial

(A)

\section{Calibration for primary endpoint}

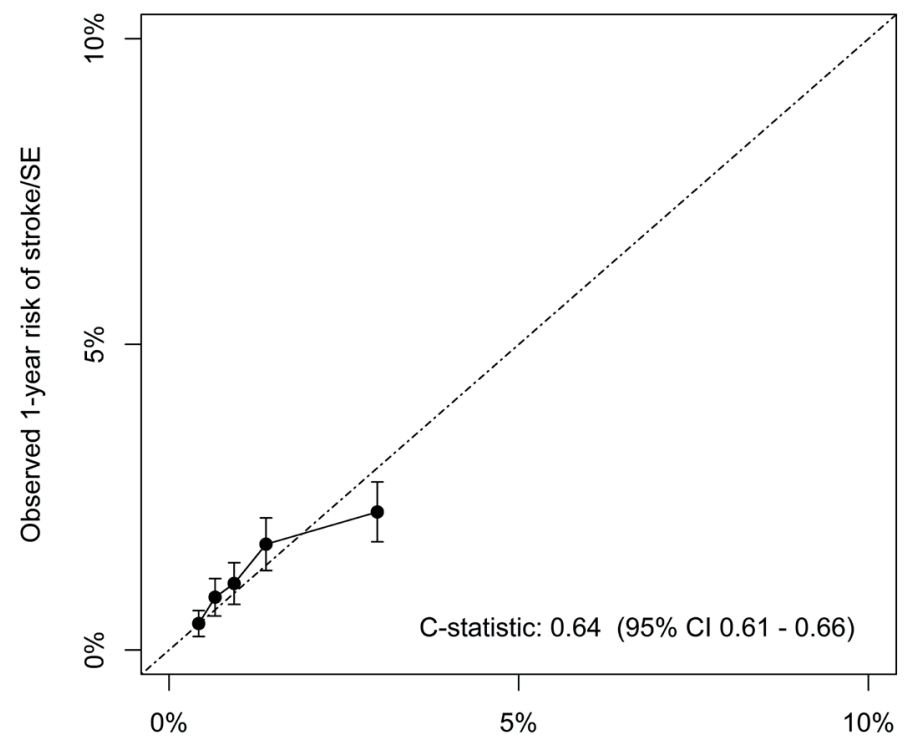

Estimated 1-year risk of stroke/SE

(B)

Calibration for major bleeding

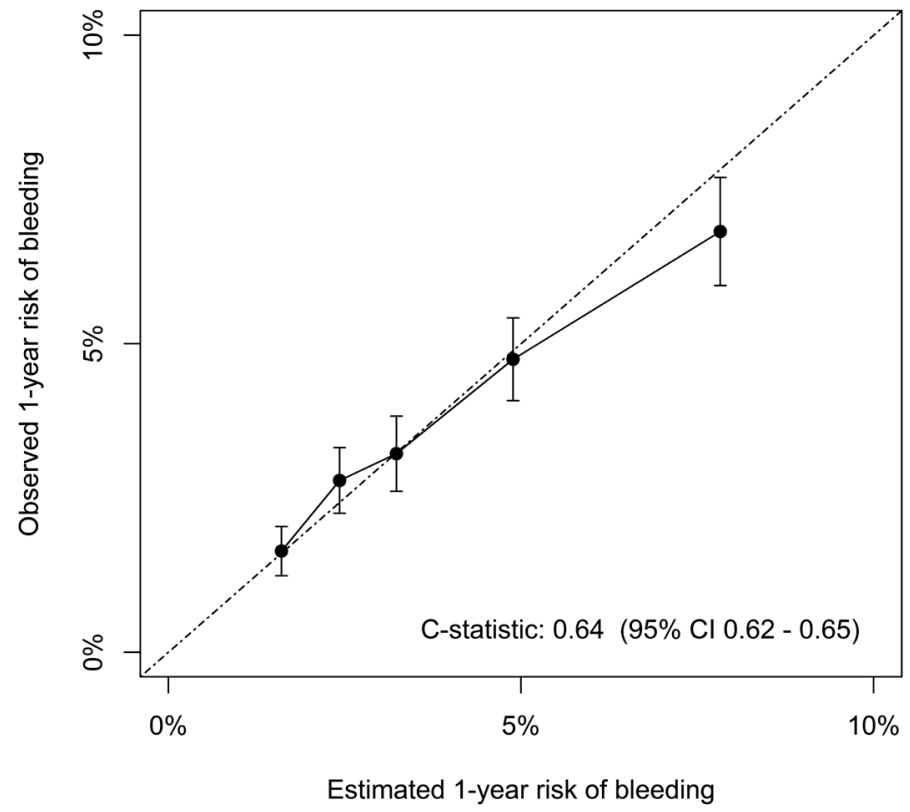


Supplemental Table 1. Baseline characteristics of the RE-LY trial stratified for quartiles of untreated baseline risk for major bleeding according to the GARFIELD-AF risk model.

\begin{tabular}{|c|c|c|c|c|}
\hline & $\begin{array}{l}\text { Quartile } 1 \\
n=62\end{array}$ & $\begin{array}{l}\text { Quartile } 2 \\
\mathrm{n}=72\end{array}$ & $\begin{array}{l}\text { Quartile } 3 \\
n=74\end{array}$ & $\begin{array}{l}\text { Quartile } 4 \\
\mathrm{n}=80\end{array}$ \\
\hline Baseline risk of bleeding, median (IQR) & $1.9(1.5-2.0)$ & $2.7(2.4-2.9)$ & $4.0(3.6-4.6)$ & $6.7(5.9-7.9)$ \\
\hline Baseline risk of stroke/SE, median (IQR) & $0.9(0.6-1.2)$ & $1.2(0.8-1.5)$ & $1.6(1.1-2.4)$ & $2.7(1.8-3.8)$ \\
\hline Age (years) & $62 \pm 7$ & $72 \pm 4$ & $74 \pm 5$ & $80 \pm 4$ \\
\hline Male sex & $3533 \pm 70$ & $2846 \pm 68$ & $2774 \pm 63$ & $2361 \pm 53$ \\
\hline \multicolumn{5}{|l|}{ Ethnicity } \\
\hline Caucasian & 3300 (66\%) & 3171 (75\%) & $2969(68 \%)$ & $3176(71 \%)$ \\
\hline Black & $62(1 \%)$ & $33(1 \%)$ & $46(1 \%)$ & $35(1 \%)$ \\
\hline Hispanic & $225(5 \%)$ & $148(4 \%)$ & $251(6 \%)$ & $255(6 \%)$ \\
\hline Other & $1434(29 \%)$ & 852 (20\%) & $1132(26 \%)$ & $1024(23 \%)$ \\
\hline Current smoking & 1750 (35\%) & $1487(35 \%)$ & $1456(33 \%)$ & $1286(29 \%)$ \\
\hline History of cardiovascular disease & 217 (4\%) & $556(13 \%)$ & $1232(28 \%)$ & 1447 (32\%) \\
\hline Systolic blood pressure (mmHg) & $130 \pm 17$ & $132 \pm 17$ & $131 \pm 18$ & $131 \pm 18$ \\
\hline Body mass index $\left(\mathrm{kg} / \mathrm{m}^{2}\right)$ & $31 \pm 6$ & $30 \pm 6$ & $28 \pm 5$ & $26 \pm 5$ \\
\hline $\begin{array}{l}\text { Estimated glomerular filtration rate }(\mathrm{ml} / \\
\left.\mathrm{min} / 1.73 \mathrm{~m}^{2}\right)\end{array}$ & $96 \pm 29$ & $81 \pm 20$ & $65 \pm 18$ & $48 \pm 10$ \\
\hline Use of oral anticoagulants at baseline & 3076 (61\%) & $2773(66 \%)$ & $2733(62 \%)$ & 2607 (58\%) \\
\hline
\end{tabular}

All data are shown as $n(\%)$ or mean \pm standard deviation unless stated otherwise; $I Q R=$ interquartile range 
Supplemental Figure 3. The relative effect of dabigatran (in doses of $110 \mathrm{mg}$ or $150 \mathrm{mg}$ twice daily) versus warfarin on the risk of major cardiovascular events in quartiles of baseline risk of major bleeding according to the GARFIELD-AF risk model

(A)

Dabigatran $110 \mathrm{mg}$

Quartile $1 \quad 0.9 \quad 37$

Quartile $2 \quad 1.2 \quad 30$

Quartile $3 \quad 1.6 \quad 41$

Quartile 4

2.7

63
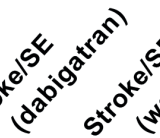

Hazard ratio

28

31

36
( $95 \%$ confidence interval)

65

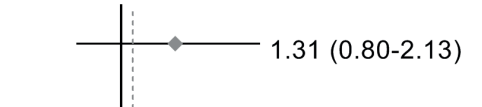

0.39

$0.96(0.58-1.58)$

$1.16(0.74-1.82)$

$0.95(0.67-1.34)$

interaction

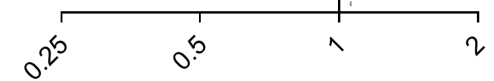

\section{Dabigatran $150 \mathrm{mg}$}

$\begin{array}{llll}\text { Quartile } 1 \quad 0.9 & 24 & 28\end{array}$

$\begin{array}{llll}\text { Quartile } 2 & 1.2 & 17 & 31\end{array}$

$\begin{array}{llll}\text { Quartile } 3 & 1.6 & 34 & 36\end{array}$

$\begin{array}{llll}\text { Quartile } 4 \quad 2.7 & 49 & 65\end{array}$

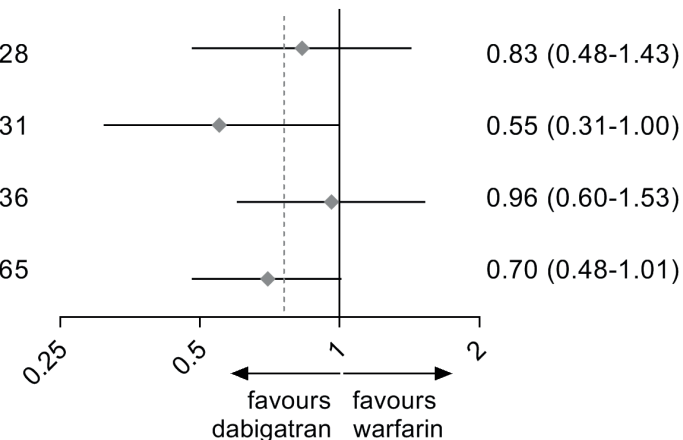




\section{References in supplemental material}

1. Wilson PW, D'Agostino RB, Levy D, Belanger AM, Silbershatz $H$, Kannel WB. Prediction of coronary heart disease using risk factor categories. Circulation. 1998 May;97(18):1837-47.

2. Conroy RM, Group on behalf of the S project, Pyörälä K, Group on behalf of the S project, Fitzgerald AP, Group on behalf of the S project, et al. Estimation of ten-year risk of fatal cardiovascular disease in Europe: the SCORE project. Eur Heart J. 2003 Jun 1;24(11):987-1003.

3. Goff DCJ, Lloyd-Jones DM, Bennett G, Coady S, D'Agostino RB, Gibbons R, et al. 2013 ACC/AHA Guideline on the Assessment of Cardiovascular Risk: A Report of the American College of Cardiology/ American Heart Association Task Force on Practice Guidelines. J Am Coll Cardiol. 2014;63(25):S49-73.

4. Kaasenbrood L, Bhatt DL, Dorresteijn JA, Wilson PW, D'Agostino RB, Massaron JM, et al. Estimated life-expectancy without recurrent cardiovascular events in patients with vascular disease: the REACHSMART model. J Am Hear. 2018;7:e009217.

5. Jaspers NEM, Ridker PM, Dorresteijn JAN, Visseren FLJ. The prediction of therapy-benefit for individual cardiovascular disease prevention: rationale, implications, and implementation. Curr Opin Lipidol. 2018;29(6):436-44.

6. Steyerberg EW. Clinical prediction models: a practical approach to development, validation and updating. New York, USA: Springer; 2009. 


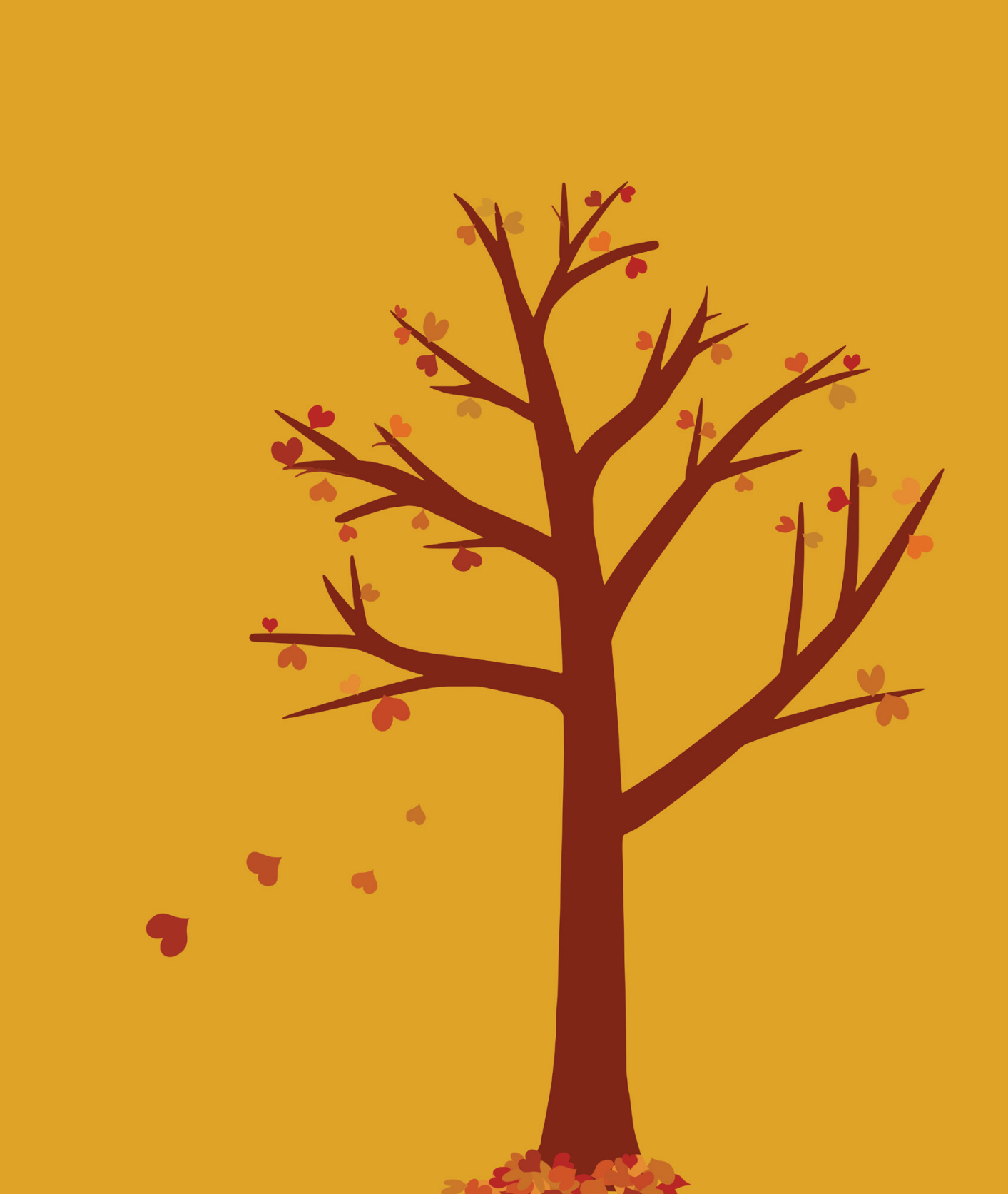




\title{
Estimating absolute cardiovascular disease
} risk in individual persons aged 65 years or older using an older person-specific risk score

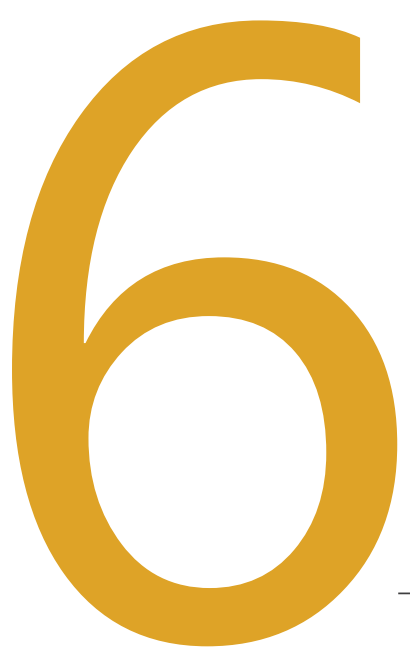

\author{
Tamar I. de Vries \\ Ruth Peters \\ Kunihiro Matsushita \\ Michael J. Blaha \\ Nigel Beckett \\ Jan Westerink
}

Jannick A.N. Dorresteijn

Marielle H. Emmelot-Vonk Majon Muller Riitta Antikainen Yolanda van der Graaf

Vijay Nambi

Chris J. Bulpitt

Frank L.J. Visseren

Manuscript draft. 


\section{ABSTRACT}

\section{Background}

Older persons are, on average, at high risk of cardiovascular disease (CVD). There is, however, a wide range in individual CVD risk in older persons and currently available risk models generally perform poorly in older persons as these models usually do not take competing risk into account and because the relationship between classical risk factors and CVD attenuates with age. The aim of this study was to validate an existing older person-specific risk score in a large population of patients aged over 65 with and without vascular disease for the prediction of CVD risk. A secondary aim was to estimate the absolute treatment effect of blood pressure (BP)-lowering in individual older persons.

\section{Methods}

An existing competing-risk adjusted older person-specific risk score was externally validated in 21,890 patients aged 65-90 from ARIC $(n=5,921)$, MESA $(n=2,983)$, SMART $(n=3,761)$, HYVET $(n=3,669)$, and SPRINT $(n=5,556)$ for the prediction of major cardiovascular events (3-point MACE; non-fatal myocardial infarction and stroke, and cardiovascular mortality) and 3-point MACE-plus-hospitalization for heart failure (MACE$\mathrm{HF}$ ). The estimated effect of BP-lowering was added to the model, based on an individual's baseline systolic BP (SBP), assuming a risk reduction of $20 \%$ per $10 \mathrm{mmHg}$ intended SBP reduction and a target SBP of $<135 \mathrm{mmHg}$.

\section{Results}

The models calibrated well for MACE and MACE-HF, with C-statistics for discrimination of 0.68 (95\% confidence interval [CI] 0.67-0.69) for both outcomes. The median 10-year risk of MACE and MACE-HF were 19.3\% (interquartile range [IQR] 13.1-27.5) and 23.3\% (IQR 15.6-33.7) respectively. Simple risk charts were constructed for ease of use in clinical practice. The median estimated 10-year absolute risk reduction for the risk of MACE for hypertensive older persons was 8.0\% (IQR 3.0-12.9).

\section{Conclusion}

The older person-specific risk score predicts the risk of MACE and MACE-HF well in a population of older persons over 65 years old without and with vascular disease. There is a wide range in predicted individual CVD risk in older persons. The older person-specific risk score can be used to predict treatment effects from cardiovascular risk management such as BP-lowering treatment in older persons, which can be used for shared-decision making. 


\section{Background}

Age is one of the strongest risk factors for cardiovascular events, and as such the 10 year risk of cardiovascular disease (CVD) is generally high at higher age. ${ }^{1}$ In clinical practice guidance on cardiovascular risk assessment in older persons is needed to balance potential benefits and harms in this population. Although international guidelines acknowledge this need, evidence based guidance is lacking and clinicians are advised to use shared decision making in selecting patients for therapy., Because of a decreasing life-expectancy and an increasing risk of non-CVD mortality with increasing age, the treatment of important cardiovascular risk factors does not necessarily translate to a high absolute treatment benefit in this population. 4,5 Thus, there is a potential risk of overtreatment, leading to polypharmacy, an increased risk of drug interactions, adverse events, reduced quality of life and unnecessary costs especially in older persons with more co-morbidities and potentially without the expected absolute benefits. ${ }^{6}$

Cardiovascular risk calculators estimating absolute treatment effect for individual patients may greatly aid in patient-centered clinical decision making. ${ }^{7}$ Unfortunately, classic CVD risk prediction models generally have a poor performance in older persons, ${ }^{8-11}$ because the relative relationship between classical risk factors and CVD attenuates with age, ${ }^{12}$ and because traditional prediction models do not take competing risk of non-CVD mortality into account. As this risk is especially high in older persons, this leads to overestimation of the CVD risk and consequently overestimation of potential treatment benefit from risk factor treatment in older persons, 5,13,14 leading to unnecessary treatment in older persons. To deal with short-comings of traditional risk models, an older person-specific risk score was previously developed in older persons aged 70 years or older for estimating CVD risk. ${ }^{4}$ The older person-specific risk score takes competing risks of non-CVD mortality into account and was initially developed to predict the absolute effect from statin treatment in older persons.

In the current study, we aimed to further optimize the older person-specific risk score for individuals aged 65 years or older ${ }^{2,3}$ and to externally validate the model in large real-life cohorts of older individuals with and without vascular disease at baseline. Additionally, incident heart failure was included in the outcome, as this is an emerging clinical condition with high incidence in older persons and with high impact, causing loss in quality of life in older persons. ${ }^{15,16}$ Finally, as an example, the absolute risk reductions from antihypertensive treatment in individual older persons was estimated with the older person-specific risk score to show the clinical benefit from using this score in daily practice. 


\section{Methods}

\section{Sources of data}

The Atherosclerosis Risk in Communities (ARIC) study is a prospective cohort study including 15,792 participants of 45-64 years of age in 1987-1989, selected through population sampling from 4 communities in the USA. ${ }^{17}$ For the current study, we used data from visit 5 (inclusion 2011-2013) to include more patients aged over 65 years.

The Hypertension in the Very Elderly Trial (HYVET; trial registration number: NCT00122811; enrollment 2001-2007) randomized 3,845 patients of 80 years of age or older with persistent hypertension (defined as a sustained systolic blood pressure (SBP) of 160 $\mathrm{mmHg}$ or higher) from 195 centers in 13 countries to either indapamide, and if necessary perindopril, to achieve a target blood pressure of below $150 / 80 \mathrm{~mm} \mathrm{Hg}$ or matching placebo. ${ }^{18}$

The Multi-Ethnic Study of Atherosclerosis (MESA) study is a multi-center prospective cohort study investigating subclinical CVD including 6,814 participants aged 45-84 between in 2000-2002, recruited from 6 communities in the USA. ${ }^{19}$

The Second Manifestations of ARTerial disease (SMART) study is an ongoing prospective cohort study at the University Medical Center Utrecht, the Netherlands, which enrolled patients aged 18 years or older with CVD or cardiovascular risk factors from 1996 onward. ${ }^{20}$ The Systolic Blood Pressure Intervention Trial (SPRINT; NCT01206062; enrollment 20102013) was a multicenter clinical trial, including a subgroup of 2,636 participants aged over 75 years, randomizing participants to a SBP target of less than $120 \mathrm{mmHg}$ versus a target of less than $140 \mathrm{mmHg}{ }^{21}$

All included studies comply with the Declaration of Helsinki, were approved by institutional review boards and all participants provided written informed consent. The current study was conducted using data from individuals aged between 65 years and 90 years from multiple trials and cohort studies, as there were few individuals aged over 90 years. Patients with no information on follow-up were excluded from analysis (Figure 1).

\section{Statistical analysis}

Missing values were imputed using predictive mean matching (areglmpute-algorithm in $\mathrm{R}$, Hmisc-package). Continuous predictors were truncated at the $1^{\text {st }}$ and $99^{\text {th }}$ percentile to minimize the influence of outliers in the model. ${ }^{22}$ All analyses were conducted with $R$ statistical software V.3.5.1 (r-project.org).

\section{Validation of the original older person-specific risk score}

The original older person-specific risk score is a Fine and Gray competing risk (i.e. non-vascular death) adjusted model for the prediction of 5- or 10-year risk of major cardiovascular events (MACE; a composite endpoint of stroke, myocardial infarction, and 
Figure 1. Patient selection

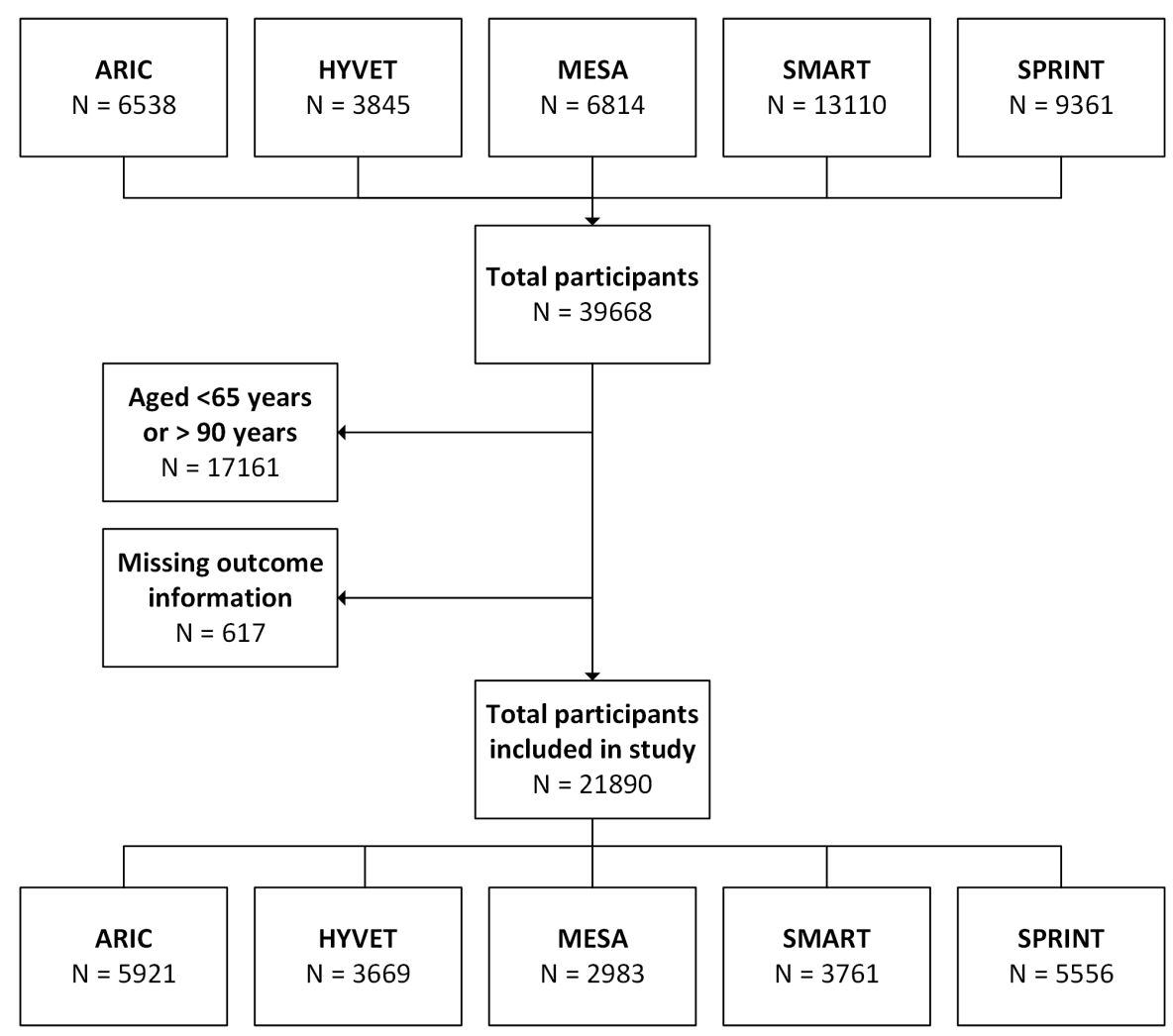

cardiovascular mortality) in older persons. ${ }^{4}$ It was developed in the PROspective Study of Pravastatin in Elderly at Risk trial population in patients with and without vascular disease aged $\geq 70$ years, ${ }^{23}$ and externally validated in the SMART cohort for patients with vascular disease, ${ }^{20}$ and in the Anglo-Scandinavian Cardiac Outcomes Trial - Lipid-Lowering Arm trial for patients without vascular disease. ${ }^{24}$ The model is based on the following baseline predictors: age, sex, current smoking, type 2 diabetes mellitus, total number of medications used, SBP, low-density lipoprotein (LDL)-cholesterol, high-density lipoprotein (HDL)cholesterol, estimated glomerular filtration rate (eGFR) assessed with the Modifcation of Diet in Renal Disease formula, and polyvascular disease (i.e. the presence of CVD at more than one location for coronary artery disease, cerebrovascular disease, or peripheral artery disease). ${ }^{4}$ The full model is shown in Supplementary Table 1. As LDL cholesterol was not measured in the HYVET study, we used approximated LDL cholesterol by non-HDL cholesterol minus $0.8 \mathrm{mmol} / \mathrm{L}^{25}$

In the current study, we externally validated the original older person-specific risk score in the included study populations of patients aged between 65 and 90 years. Performance of the model for the prediction of MACE in the study populations was assessed by visual 
inspection of calibration plots of the predicted versus observed risk of MACE, and in terms of discrimination using the c-statistic. To optimally estimate vascular risk for individual patients, we adjusted for differences in baseline risk between the study populations by recalibration of the baseline risk using the expected-versus-observed ratio if the study populations were not from Scotland or Ireland of the rest of North- or West-Europe, as the older person-specific risk score has previously only been validated for use in these regions. ${ }^{4}$ For the trials, recalibration was performed in the control arm of the trial, with adjustment for treatment effect of the trial intervention by adding the trial effect to the model for calibration of the active treatment arm of the trial.

Given that incident heart failure is a major cause of morbidity and mortality in older persons, we then updated the composite cardiovascular endpoint of the model to include hospitalization from heart failure (MACE-HF). As the incidence rates of the updated composite endpoint were higher than of the original composite endpoint, recalibration of the model was performed to account for differences in baseline risk using the expectedversus-observed ratio derived from the MESA cohort, and SPRINT trial to include enough patients with baseline CVD. As heart failure was not collected systematically throughout the whole follow-up of SMART, the SMART participants were excluded for this analysis as to minimize the risk of bias. The predictive value of the model for the updated endpoint was assessed using the C-statistic for discrimination and observed-versus-predicted survival plots for calibration.

Additional updates and validation of the older person-specific risk score

Further validation was performed for subgroups of patients based on sex, history of CVD, and age (65-80 years old versus $\geq 80$ years old). In order to allow clinicians to use additional prognostic information that may be available in clinical practice to improve the prognostic value of the model, we further added the option to include additional prognostic factors to the model using the naive method. The methodology of this is described in full in Supplementary Methods 1 . These prognostic factors include frailty according to different frailty indices, pulse pressure, ankle brachial index, albuminuria, high sensitivity-CRP, hematocrit, left ventricular hypertrophy on ECG, and intima media thickness.

\section{Older person-specific risk score charts}

A simplified version of the older person-specific risk score was created to allow the use of a simple risk chart for easy use in clinical practice. Because only five variables can be used in these two-dimensional risk charts, the following assumptions were made: the population mean of $\mathrm{HDL}$-cholesterol of $1.2 \mathrm{mmol} / \mathrm{L}$ in men and $1.4 \mathrm{mmol} / \mathrm{L}$ in women; and the population median of an eGFR of $70 \mathrm{ml} / \mathrm{min} / 1.73,5$ medications used in total, no diabetes mellitus, and (in case of a history of CVD) no polyvascular disease. Risk charts were created for patients with and without CVD at baseline. The risk chart includes 
estimations of 10 -year risk of fatal CVD (using a recalibration of the older person-specific risk score accounting for the baseline risk for fatal events), but can also be used the estimate the 10-year risk of MACE and MACE-HF.

Estimating treatment effect of blood pressure lowering

To show the potential use of the updated older person-specific risk score in daily practice we included analyses on the individual absolute benefit of blood pressure lowering in older persons. To estimate the effect of blood pressure lowering on MACE and MACE$H F$, average relative treatment effects were added to the older person-specific risk score. The relative treatment effect was derived from a meta-analysis with 613,815 participants from randomized controlled trials of blood pressure lowering treatment, including trials with older persons (>70 years), which reported a hazard ratio of 0.80 per $10 \mathrm{mmHg}$ SBP reduction. ${ }^{26}$ Treatment benefit was calculated using this hazard ratio for the reduction of office SBP of the individual patient with an indication for blood pressure lowering treatment at baseline to the target of $130-140 \mathrm{mmHg}$, using $135 \mathrm{mmHg}$ as an estimated treatment target, according to the European Society of Cardiology/European Society of Hypertension (ESC/ESH) 2018 guidelines for the management of arterial hypertension. ${ }^{2}$ An indication for blood pressure lowering therapy was defined as an SBP $>140 \mathrm{mmHg}$ at baseline for patients aged 65 or older, and $>160 \mathrm{mmHg}$ at baseline for patients aged 80 or older, according to the ESC/ESH guidelines. ${ }^{2}$ Treatment benefit for individual patients was defined as the patient's predicted absolute 5- or 10-year risk of MACE or MACE-HF according to the older person-specific risk score minus the patient's absolute risk of MACE or MACE-HF when lowering SBP to $140 \mathrm{mmHg}$, and was expressed as an absolute risk reduction (ARR). In the main paper, 10-year risks are presented, while 5-year predictions can be found in the Supplemental Material, as 5-year risks may seemingly underestimate the true risks and treatment effects in older persons, as life expectancy is still on average 8 to 10 years even in 80 -year-olds. ${ }^{27}$

Subgroup analyses for the treatment effects from blood pressure lowering were performed stratified for sex. Finally, a sensitivity analysis was performed including only patients on lipid-lowering medication at baseline, as lipid-lowering treatment will influence the individual absolute baseline risk of CVD and part of the study population was included before lipid lowering therapy was common practice. 


\section{Results}

A total of 21,890 older study participants from trials and cohorts were included in the present analyses (Figure 1). Baseline characteristics of all study populations are presented in Table 1. The mean age was 75 years \pm 6 standard deviation (SD); $52 \%$ was male; $25 \%$ had a history of CVD; the mean SBP was $143 \mathrm{mmHg} \pm 23$ SD. Follow-up and trial outcomes are presented in Table 2.

\section{External validation and updates of the older person-specific risk score to predict MACE and MACE-HF}

After recalibration for baseline risk in each study population, predicted 5-year risk for MACE showed good agreement with the observed 5-year risk for MACE in the combined study population, with some underestimation in the highest risk quantiles (Figure 2a). The C-statistic of the estimated 5-year MACE risk was 0.68 (95\% confidence interval [CI] 0.67-0.69). Predicted 5-year risk of MACE-HF also showed good agreement with the observed risk, again with some underestimation in the highest risk quantiles (Figure $2 b$ ). The C-statistic of the estimated 5-year MACE-HF risk was 0.68 (95\% Cl 0.67-0.69). The calibration for subgroups based on sex, history of CVD, and age are shown in Supplemental Figure 1. The validation of adding optional prognostic variables to the model is shown in Supplemental Figure 2. Adding additional prognostic variables improves discrimination of the model for MACE (C-statistic 0.69; 95\% Cl 0.68-0.70).

The simplified two-dimensional risk charts for the older person-specific risk score for easy use in clinical practice can be found in Supplementary Figure 3, stratified for the presence of CVD at baseline.

\section{Distribution of CVD risk in individual older persons}

Figure 3 shows the distribution of individual risk of the estimated 10-year risk of respectively MACE and MACE-HF stratified for history of clinically manifest vascular disease at baseline. The median estimated 10-year risk of MACE was 19.3\% (interquartile range [IQR] 13.1-27.5, total range 3.3-90.7\%), with considerably higher risks in patients with vascular disease at baseline (median 28.6\%; IQR 21.5-38.6, total range 5.8-90.7\%) than in patients without vascular disease at baseline (median 16.6\%; IQR 11.9-23.4, total range 3.3-79.1\%). The median estimated 10-year risk of MACE-HF was 23.3\% (IQR 15.6-33.7, total range 3.9$96.7 \%)$, with again considerably higher risks in patients with vascular disease at baseline (median 38.3\%; IQR 29.4-40.4, total range 8.2-96.7\%) than in patients without vascular disease at baseline (median 19.5\%; IQR 14.0-27.4, total range 3.9-84.6\%). The distribution of 5-year risk of MACE and MACE-HF can be found in Supplemental Figure 4. 
Table 1. Patient characteristics of the study populations at baseline

\begin{tabular}{llllll}
\hline & ARIC & HYVET & MESA & SMART & SPRINT \\
& $\mathrm{n}=5921$ & $\mathrm{n}=3669$ & $\mathrm{n}=2983$ & $\mathrm{n}=3761$ & $\mathrm{n}=5556$ \\
\hline Male sex & $2348(40 \%)$ & $1463(40 \%)$ & $1421(48 \%)$ & $2620(70 \%)$ & $3471(62 \%)$ \\
Age (years) & $76 \pm 5$ & $83 \pm 2$ & $72 \pm 5$ & $70 \pm 4$ & $74 \pm 6$ \\
Current smoking & $412(7 \%)$ & $244(7 \%)$ & $231(8 \%)$ & $633(17 \%)$ & $318(6 \%)$ \\
Systolic blood pressure (mmHg) & $131 \pm 18$ & $173 \pm 9$ & $134 \pm 22$ & $147 \pm 22$ & $140 \pm 15$ \\
Diastolic blood pressure (mmHg) & $67 \pm 11$ & $91 \pm 8$ & $71 \pm 10$ & $81 \pm 12$ & $74 \pm 11$ \\
Total cholesterol (mmol/L) & $4.7 \pm 1.1$ & $5.3 \pm 1.1$ & $5.0 \pm 0.9$ & $4.9 \pm 1.2$ & $4.8 \pm 1.0$ \\
HDL-cholesterol (mmol/L) & $1.4 \pm 0.4$ & $1.3 \pm 0.4$ & $1.3 \pm 0.4$ & $1.3 \pm 0.4$ & $1.4 \pm 0.4$ \\
LDL cholesterol (mmol/L) & $2.7 \pm 0.9$ & $3.1 \pm 1.1$ & $3.0 \pm 0.8$ & $2.9 \pm 1.1$ & $2.8 \pm 0.9$ \\
eGFR (ml/min/1.73m2) & $71(58-83)$ & $64(52-78)$ & $70(60-82)$ & $68(57-80)$ & $67(54-80)$ \\
Presence of micro-albuminuria & $977(17 \%)$ & $\mathrm{N} / \mathrm{A}$ & $345(12 \%)$ & $647(17 \%)$ & $1059(19 \%)$ \\
Presence of macro-albuminuria & $149(3 \%)$ & $\mathrm{N} / \mathrm{A}$ & $62(2 \%)$ & $109(3 \%)$ & $165(3 \%)$ \\
History of cardiovascular disease & $768(13 \%)$ & $438(12 \%)$ & $6(0 \%)$ & $3164(84 \%)$ & $1096(20 \%)$ \\
$\quad$ Coronary artery disease & $422(7 \%)$ & $119(3 \%)$ & $0(0 \%)$ & $1979(53 \%)$ & $\mathrm{N} / \mathrm{A}$ \\
Cerebrovascular disease & $208(4 \%)$ & $253(7 \%)$ & $0(0 \%)$ & $934(25 \%)$ & N/A \\
History of heart failure & $589(10 \%)$ & $112(3 \%)$ & $1(0 \%)$ & N/A & N/A \\
Type 2 diabetes mellitus & $1912(32 \%)$ & $375(10 \%)$ & $461(15 \%)$ & $827(22 \%)$ & $0(0 \%)$ \\
Lipid lowering medication & $2962(50 \%)$ & $14(0 \%)$ & $663(22 \%)$ & $2357(63 \%)$ & $2815(51 \%)$ \\
Prior antihypertensive medication & $\mathrm{N} / \mathrm{A}$ & $2399(65 \%)$ & $1316(44 \%)$ & $2965(79 \%)$ & $5136(92 \%)$ \\
Number of drugs (median [IQR]) & $8(6-12)$ & $0(0-1)$ & $3(2-5)$ & $4(2-5)$ & $3(3-3)$ \\
\hline
\end{tabular}

$H D L=$ high-density lipoprotein; $L D L=$ low-density lipoprotein; $e G F R=$ estimated glomerular filtration rate; $C K D-E P I=$ Chronic Kidney Disease Epidemiology Collaboration; $I Q R=$ interquartile range All data in $n(\%)$ or mean \pm standard deviation, unless specified otherwise

Table 2. Recruitment period and endpoints per study

\begin{tabular}{llllll}
\hline & $\begin{array}{l}\text { ARIC } \\
(\mathrm{n}=5,921)\end{array}$ & $\begin{array}{l}\text { HYVET } \\
(\mathrm{n}=3,669)\end{array}$ & $\begin{array}{l}\text { MESA } \\
(\mathrm{n}=2,983)\end{array}$ & $\begin{array}{l}\text { SMART } \\
(\mathrm{n}=3,761)\end{array}$ & $\begin{array}{l}\text { SPRINT } \\
(\mathrm{n}=5,556)\end{array}$ \\
\hline Recruitment period & $\begin{array}{l}2011-2013 \\
(\text { visit } 5)\end{array}$ & $2001-2007$ & $2000-2002$ & $1996-2018$ & $2010-2013$ \\
& $5.5(4.8-5.9)$ & $1.8(1.0-2.8)$ & $13.5(7.8-14.2)$ & $6.6(3.4-10.2)$ & $3.2(2.7-3.7)$ \\
Follow-up (years) & $722(12 \%)$ & $259(7 \%)$ & $1,042(35 \%)$ & $929(25 \%)$ & $284(5 \%)$ \\
MACE events* & $858(15 \%)$ & $399(11 \%)$ & $984(33 \%)$ & $1,328(35 \%)$ & $276(5 \%)$ \\
Total mortality & $366(6 \%)$ & $73(2 \%)$ & $241(8 \%)$ & $9(0 \%)+$ & $129(2 \%)$ \\
Hospitalization for HF & $366)$ & &
\end{tabular}

MACE = major adverse cardiovascular events; $H F=$ heart failure

* MACE is defined as non-fatal myocardial infarction; non-fatal stroke; or cardiovascular mortality

+ Heart failure was only collected as endpoint variable after October 2011. 
Figure 2. External validation of the older person-specific risk score for (A) the prediction of risk for myocardial infarction (MI), stroke, or cardiovascular (CV) death (MACE); and (B) the prediction of risk for MACE plus heart failure (MACE-HF)

(A) Prediction of MACE $(n=21890)$

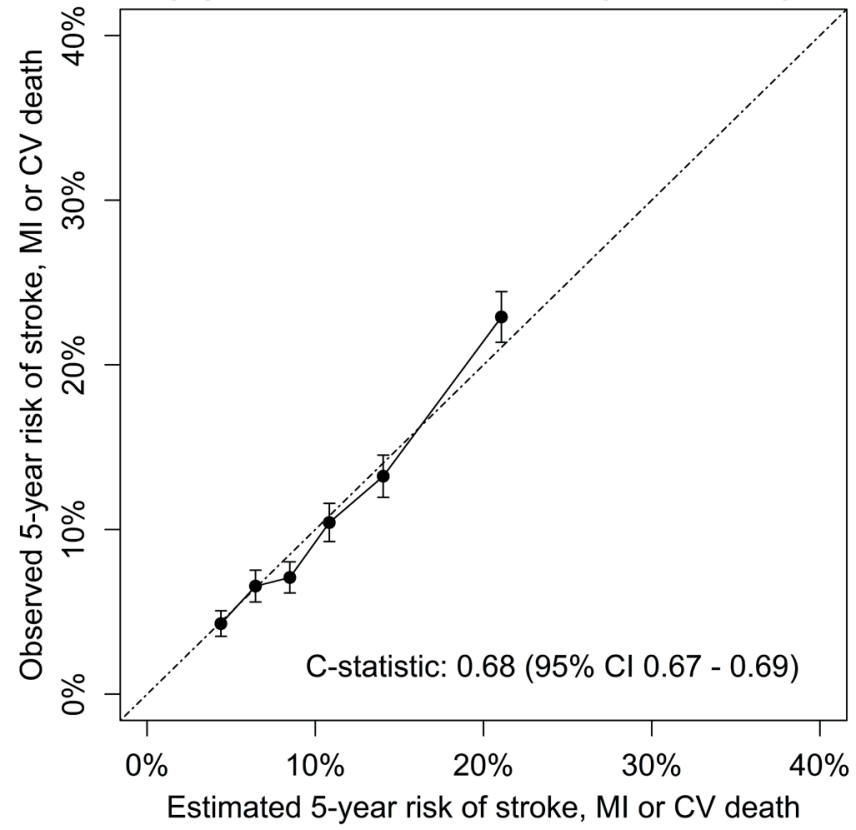

(B) Prediction of MACE-HF $(n=18129)$

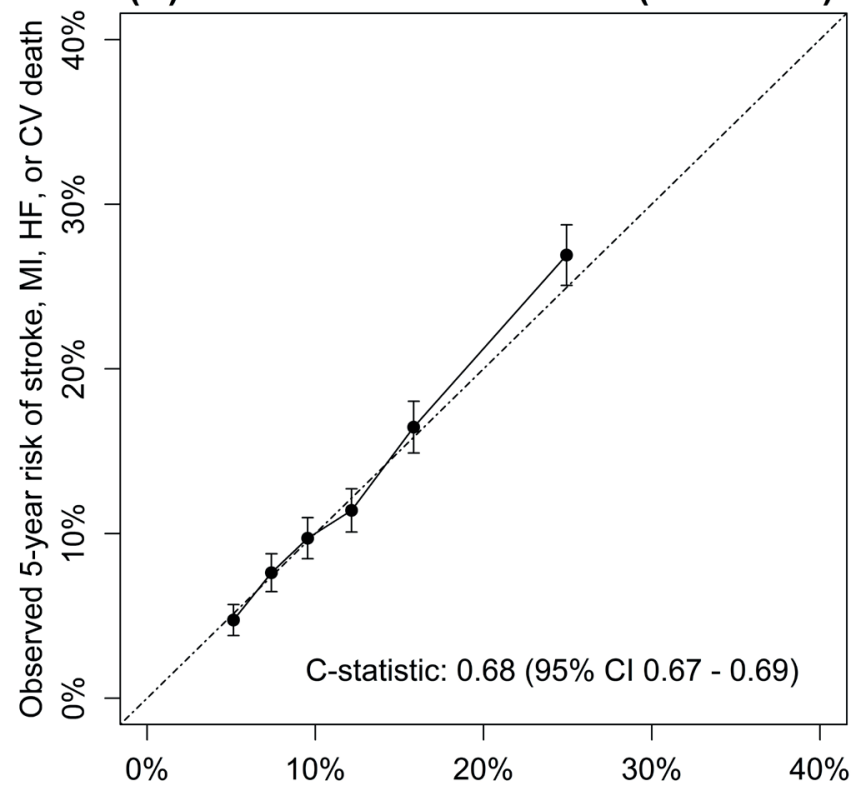

Estimated 5-year risk of stroke, MI, HF, or CV death 
Figure 3. Histograms showing the predicted 10-year risk of (A) major cardiovascular events (MACE; myocardial infarction, stroke, or cardiovascular death), and (B) MACE + heart failure (MACE-HF) in older persons without clinically manifest vascular disease at baseline; and the risk of (C) MACE, and (D) MACE-HF in older persons with clinically manifest vascular disease at baseline.
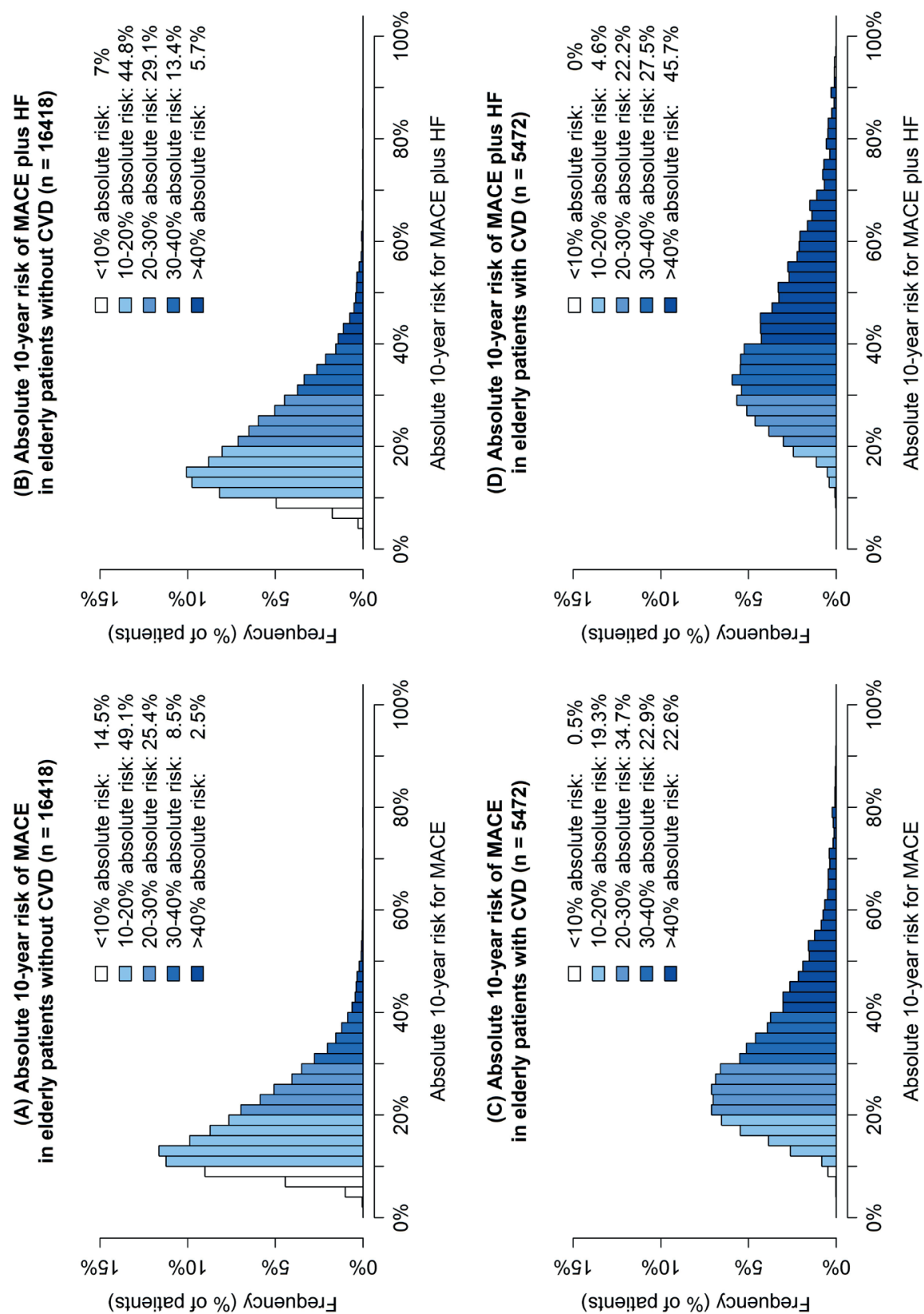
Figure 4. Histograms showing the predicted 10-year absolute risk reduction from bloodpressure lowering in older persons with an indication for blood-pressure lowering therapy for (A) major cardiovascular events (MACE; myocardial infarction, stroke, or cardiovascular death), and (B) MACE + heart failure (MACE-HF) in patients without clinically manifest vascular disease at baseline; and for (C) MACE, and (D) MACE-HF in patients with clinically manifest vascular disease at baseline.
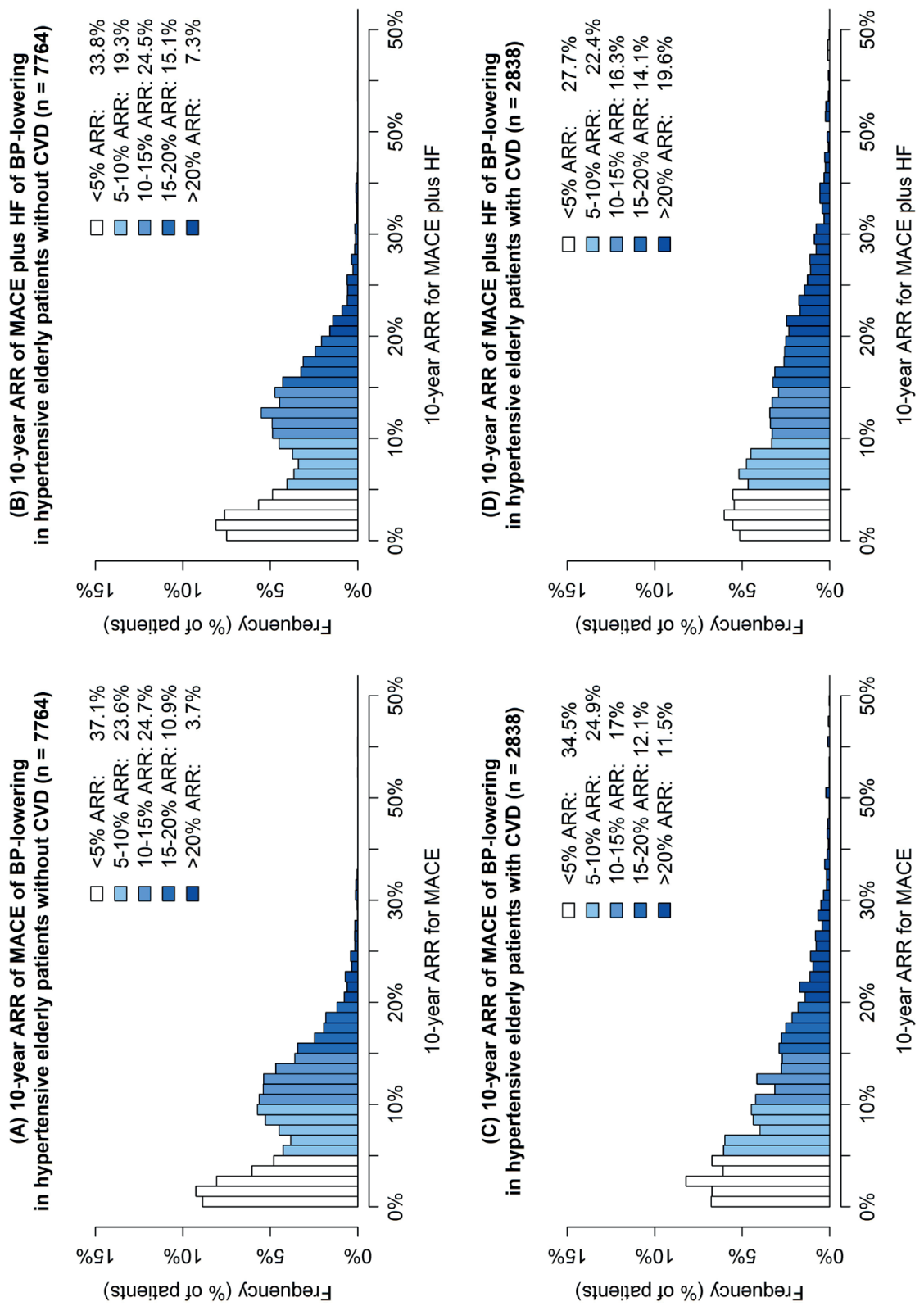


\section{Estimating treatment effect of blood pressure-lowering in older persons}

Figure 4 shows the distribution of the individual 10-year ARR for the estimated 10-year risk of respectively MACE and MACE-HF with blood pressure lowering therapy when targeting an SBP of $135 \mathrm{mmHg}$ in older persons with an indication for blood pressure lowering according to the ESC/ESH 2018 guidelines 2, stratified for history of clinically manifest vascular disease at baseline. The overall median estimated individual ARR for MACE was $8.0 \%$ (IQR 3.0-12.9); 7.8\% (IQR 3.5-14.6) and 8.1\% (IQR 2.8-12.6) in patients with and without CVD at baseline, respectively. The overall median estimated individual ARR for MACE-HF was 9.4\% (IQR 3.6-15.1); 10\% (IQR 4.5-17.8) and 9.3\% (IQR 3.3-14.4) in patients with and without CVD at baseline, respectively. The distribution of 5-year ARR from blood pressure lowering for MACE and MACE-HF can be found in Supplemental Figure 5. The results of the subgroup analysis based on sex and sensitivity analysis using only patients on lipid-lowering medication at baseline are shown in Supplemental Figure 6.

\section{Discussion}

In this study, we have validated and extended the non-CVD mortality competing-risk adjusted older person-specific risk score for the prediction of future CVD risk in patients aged between 65 and 90 years old, demonstrating the predictive reliability of the model in older persons with and without vascular disease. Individual predictions can be made for both the risk of MACE and MACE-HF, using readily available clinical characteristics. There is a wide range in predicted individual CVD risk in older persons. Using the older personspecific risk score, individualized effects of blood pressure lowering can be estimated in terms absolute risk reduction, which provides a useful example of the benefit of this approach. The full algorithm is provided in the Supplementary Materials to enable external use for researchers. The risk charts can be found in the Supplementary Material, while the full clinical tool can be accessed freely on www.U-Prevent.com.

There are various CVD risk models currently available. However, most of the widely used and recommended risk models have mainly been developed in and for middleaged populations, while the relative contribution of conventional risk factors to CVD risk decrease in the older population. ${ }^{28}$ Therefore, these models cannot be expected to give reliable prediction in older persons. Refitting a risk score in an older population using the same variables as in the Framingham risk score has been shown to improve cardiovascular risk prediction, however, these refitted models have not been externally validated. ${ }^{10,29}$ The Systematic COronary Risk Evaluation - Older Persons (SCORE-OP) ${ }^{30}$ algorithm for older persons aged 65-79 years has limited discriminative performance in an external population (0.63, 95\% Cl 0.60-0.65) 31. SCORE-OP only assesses CV death while non-fatal CVD, 
especially non-fatal stroke, is of great importance in older persons. Additionally, SCORE$\mathrm{OP}$ is only applicable in primary prevention, and has not been validated for use in patients aged 80 or older. Most importantly, an important disadvantage of existing risk scores is that they are not competing risk adjusted. Especially in older individuals, who are usually at a higher risk of competing events, this can lead to overestimations of the CVD risk, and thus overestimations of the assumed benefit of blood pressure lowering. ${ }^{5}$ The advantages of the older person-specific risk score compared to these previous models are that it was derived specifically in an older study population, and has been adjusted for competing risk of non-cardiovascular mortality. The score has now been validated for a wide age range in several populations with very different population baseline risks including both cohorts and trials, for use in patients aged between 65 and 90 years. Additionally, the model has now been updated to include hospitalization for heart failure in the composite endpoint, which is an important source of morbidity in the older population. ${ }^{15}$ Finally, an important advantage is that the model can be used to predict treatment of cardiovascular risk modification by applying the HR associated with the intervention. Implementation in clinical practice of the www.U-Prevent.com website, including the older personspecific risk score, is advised by the European Association of Preventive Cardiology, Acute Cardiovascular Care Association, and the Association of Cardiovascular Nursing and Allied Professions. $^{32}$

As an illustration of the clinical use of the older person-specific risk score, we have provided the example of the estimation of treatment effects from blood pressure lowering using the model. The ESC/ESH 2018 guidelines for the management of arterial hypertension defines older patients (the 'old') as $\geq 65$ years and the 'very old' as $\geq 80$ years, and includes different recommendations for these two age groups. In fit older patients, initiation of blood pressure-lowering treatment is justified at an SBP $\geq 140 \mathrm{mmHg}$, provided that treatment is well tolerated. In the very old, initiation of blood pressure lowering is recommended at an SBP $\geq 160 \mathrm{mmHg}$. The target in all older patients is $130-140 \mathrm{mmHg}$. Especially in the very old, it is recommended that treated SBP values of $<130$ should be avoided. ${ }^{2}$ The analyses in the current study were performed in line with these recommendations. Individual treatment effect predictions are also possible for other treatment targets. For example, the 2017 American guidelines for high blood pressure recommends treatment of hypertension with a SBP treatment goals below $130 \mathrm{mmHg}$ for all noninstitutionalized ambulatory community-dwelling adults over 65 years old with an average SBP of $130 \mathrm{~mm}$ Hg or higher. ${ }^{3}$

The ESC/ESH guideline further notes that older people are invariably at high absolute risk for $C V D,{ }^{2}$ thereby deserving of blood pressure lowering therapy if hypertension is present, while the American guideline notes that the risk increase due to high blood pressure is larger in older adults given the higher absolute risk of CVD at an older age. ${ }^{3}$ The current 
study confirms that in older persons with an indication for blood pressure lowering therapy according to the ESC/ESH guideline, absolute 10-year risk for MACE is generally very high, being $>10 \%$ in $90 \%$ of the study population, and even $>20 \%$ in around $50 \%$ of the study population. On average, the risk is higher in patients with CVD at baseline when compared to those without CVD at baseline. However, even in this high risk population, there is a wide distribution in the therapy benefit from blood pressure lowering to 135 $\mathrm{mmHg}$ in those with an indication for therapy, from just $1 \%$ to more than $50 \%$ estimated 10 -year ARR leading to numbers needed to treat over 10 years between 100 to only 2 . This large difference is solely based on both the difference in baseline (untreated) risk and the height of SBP at baseline. Thus, while blood pressure lowering is likely worthwhile in the vast majority of very older persons with sustained hypertension, there are individuals with potentially less benefit. In these patients, the potential benefit from blood pressure lowering might not outweigh potential harms. The predictions from the older personspecific risk score can be used in shared clinical decision making, and for communicating the benefit of blood pressure lowering to older persons.

Several potential limitations of the current study should be acknowledged. First, the calibration plots show some underestimation of CVD risk in the highest risk quantiles. However, this underestimation only occurs in very high risk patients, with a 5 -year risk of CVD of higher than 20\% (a 10-year risk of about $35 \%$ or higher), while in patients with a lower risk, the risk estimation is very reliable. This is clinically the most relevant patient group, as these are the patients in which there may be questions regarding the benefit of cardiovascular risk management. Second, the C-statistics of the model are moderate. Addition of extra prognostic variables to the model using the naïve method improves the C-statistic to 0.69. Furthermore, as mentioned before, the calibration of the risk model shows reliable estimations for those patients where questions regarding cardiovascular risk management may exist. As reliability of the predicted probabilities influences treatment decisions, calibration may be a clinically more relevant metric than discrimination for the purpose of clinical decision making. ${ }^{33} \mathrm{~A}$ third potential limitation is that a single treatment effect for blood pressure lowering has been used for all patients. It is assumed that the relative treatment effect is the same for all patients, and dependent on the intended decrease in SBP only. In the current study no heterogeneity of treatment effect was found across the baseline risk for disease in the HYVET and SPRINT trials, as assessed by fitting a survival model including an interaction term between the linear predictor and treatment allocation. Therefore, there is no evidence to suggest that this assumption does not hold. Furthermore, an important potential limitation is that very frail older persons may not be well-represented in the included studies, especially the trials, which may include healthier individuals than representative for the general population. ${ }^{34,35}$ However, the burden of frailty in HYVET participants was found to be similar to that 
seen in population-based studies and post-hoc analyses of HYVET have shown that the relative benefit of blood pressure lowering may be larger in frailer adults. ${ }^{36}$ Also, frailty status can be added as an additional prognostic variable to the model using the naïve method, to improve the predictive value of the risk model. On the other hand, in very frail older persons, caution should be exercised with risk factor treatment due to a risk of adverse events. If life expectancy is likely limited, one should question whether using risk estimation has added value, or alternatively, the 5-year risk can be assessed. Finally, an inherent limitation of absolute risk estimations, is that older individuals are invariably at higher risk for CVD than younger individuals with the same risk factors. As higher risk translates to higher absolute risk reductions, this gives the impression that risk factors should be treated more in the oldest persons. Therefore, in the future it might be interest to focus more on lifetime benefit from risk factor treatment, ${ }^{37-39}$ which shifts the focus of treatment to younger individuals.

\section{Conclusion}

The older person-specific risk score predicts well in a population of older persons over 65 years old without and with vascular disease. There is a wide range in predicted individual CVD risk in older persons. The older person-specific risk score can also be used to predict treatment effects from cardiovascular risk management such as BP-lowering treatment in older persons, which can be used for shared-decision making before treating CV risk factors. 


\section{References}

1. North BJ, Sinclair DA. The intersection between aging and cardiovascular disease. Circ Res. 2012;110(8):1097-108.

2. Williams B, Mancia G, Spiering W, Agabiti Rosei E, Azizi M, Burnier M, et al. 2018 ESC/ESH Guidelines for the management of arterial hypertension. Eur Heart J. 2018;39(33):3021-104.

3. Whelton PK, Carey RM, Aronow WS, Casey DEJ, Collins KJ, Dennison Himmelfarb C, et al. 2017 ACC/AHA/AAPA/ABC/ACPM/AGS/APhA/ASH/ASPC/NMA/PCNA Guideline for the Prevention, Detection, Evaluation, and Management of High Blood Pressure in Adults: Executive Summary: A Report of the American College of Cardiology/American Heart Association Task F. J Am Coll Cardiol. 2018;71(19):2199-269.

4. Stam-Slob MC, Visseren FLJ, Jukema J, van der Graaf Y, Poulter NR, Gupta A, et al. Personalized absolute benefit of statin treatment for primary or secondary prevention of vascular disease in individual elderly patients. Clin Res Cardiol. 2017;106(1):58-68.

5. Wolbers M, Koller MT, Witteman JCM, Steyerberg EW. Prognostic models with competing risks: methods and application to coronary risk prediction. Epidemiology. 2009;20(4):555-61.

6. Field TS, Gurwitz JH, Harrold LR, Rothschild J, DeBellis KR, Seger AC, et al. Risk factors for adverse drug events among older adults in the ambulatory setting. J Am Geriatr Soc. 2004;52(8):134954.

7. Kent DM, Steyerberg E, van Klaveren D. Personalized evidence based medicine: predictive approaches to heterogeneous treatment effects. BMJ. 2018;363:k4245.

8. Sabayan B, Gussekloo J, de Ruijter W, Westendorp RGJ, de Craen AJM. Framingham stroke risk score and cognitive impairment for predicting first-time stroke in the oldest old. Stroke. 2013;44(7):1866-71.

9. de Ruijter W, Westendorp RGJ, Assendelft WJJ, den Elzen WPJ, de Craen AJM, le Cessie S, et al. Use of Framingham risk score and new biomarkers to predict cardiovascular mortality in older people: population based observational cohort study. BMJ. 2009;338:a3083.

10. Rodondi N, Locatelli I, Aujesky D, Butler J, Vittinghoff E, Simonsick E, et al. Framingham risk score and alternatives for prediction of coronary heart disease in older adults. PLoS One. 2012;7(3):e34287.

11. Nanna MG, Peterson ED, Wojdyla D, Navar AM. The Accuracy of Cardiovascular Pooled Cohort Risk Estimates in U.S. Older Adults. J Gen Intern Med. 2019 Oct;

12. Kannel WB, D'Agostino RB. The Importance of Cardiovascular Risk Factors in the Elderly. Am J Geriatr Cardiol. 1995;4(2):10-23.

13. Austin PC, Lee DS, Fine JP. Introduction to the Analysis of Survival Data in the Presence of Competing Risks. Circulation. 2016;133(6):601-9.

14. Berry SD, Ngo L, Samelson EJ, Kiel DP. Competing risk of death: an important consideration in studies of older adults. J Am Geriatr Soc. 2010;58(4):783-7.

15. Mozaffarian D, Benjamin EJ, Go AS, Arnett DK, Blaha MJ, Cushman M, et al. Executive Summary: 
Heart Disease and Stroke Statistics--2016 Update: A Report From the American Heart Association. Circulation. 2016;133(4):447-54.

16. Bui AL, Horwich TB, Fonarow GC. Epidemiology and risk profile of heart failure. Nat Rev Cardiol. 2011;8(1):30-41.

17. The Atherosclerosis Risk in Communities (ARIC) Study: design and objectives. The ARIC investigators. Am J Epidemiol. 1989;129(4):687-702.

18. Bulpitt C, Fletcher A, Beckett N, Coope J, Gil-Extremera B, Forette F, et al. Hypertension in the Very Elderly Trial (HYVET): protocol for the main trial. Drugs Aging. 2001;18(3):151-64.

19. Bild DE, Bluemke DA, Burke GL, Detrano R, Diez Roux A V, Folsom AR, et al. Multi-Ethnic Study of Atherosclerosis: objectives and design. Am J Epidemiol. 2002;156(9):871-81.

20. Simons PC, Algra A, van de Laak MF, Grobbee DE, van der Graaf Y. Second manifestations of ARTerial disease (SMART) study: rationale and design. Eur J Endocrinol. 1999;15(9):773-81.

21. Ambrosius WT, Sink KM, Foy CG, Berlowitz DR, Cheung AK, Cushman WC, et al. The design and rationale of a multicenter clinical trial comparing two strategies for control of systolic blood pressure: the Systolic Blood Pressure Intervention Trial (SPRINT). Clin Trials. 2014;11(5):532-46.

22. Steyerberg EW. Clinical prediction models: a practical approach to development, validation and updating. New York, USA: Springer; 2009.

23. Shepherd J, Blauw GJ, Murphy MB, Bollen ELEM, Buckley BM, Cobbe SM, et al. Pravastatin in elderly individuals at risk of vascular disease (PROSPER): a randomised controlled trial. Lancet. 2002;360(9346):1623-30.

24. Dahlof B, Sever PS, Poulter NR, Wedel H, Beevers DG, Caulfield M, et al. Prevention of cardiovascular events with an antihypertensive regimen of amlodipine adding perindopril as required versus atenolol adding bendroflumethiazide as required, in the Anglo-Scandinavian Cardiac Outcomes Trial-Blood Pressure Lowering Arm (ASCOT-B. Lancet. 2005 Sep;366(9489):895-906.

25. Piepoli MF, Hoes AW, Agewall S, Albus C, Brotons C, Catapano AL, et al. 2016 European guidelines on cardiovascular disease prevention in clinical practice: the Sixth Joint Task Force of the European Society of Cardiology and Other Societies on Cardiovascular Disease Prevention in Clinical Practice. Eur Heart J. 2016;37(29):2315-81.

26. Ettehad D, Emdin CA, Kiran A, Anderson SG, Callender T, Emberson J, et al. Blood pressure lowering for prevention of cardiovascular disease and death: a systematic review and metaanalysis. Lancet. 2016;387(10022):957-67.

27. Thinggaard M, McGue M, Jeune B, Osler M, Vaupel JW, Christensen K. Survival Prognosis in Very Old Adults. J Am Geriatr Soc. 2016;64(1):81-8.

28. Bambrick P, Tan WS, Mulcahy R, Pope GA, Cooke J. Vascular risk assessment in older adults without a history of cardiovascular disease. Exp Gerontol. 2016;79:37-45.

29. Simons LA, Simons J, Friedlander Y, McCallum J, Palaniappan L. Risk functions for prediction of cardiovascular disease in elderly Australians: the Dubbo Study. Med J Aust. 2003;178(3):113-6.

30. Cooney MT, Selmer R, Lindman A, Tverdal A, Menotti A, Thomsen T, et al. Cardiovascular risk estimation in older persons: SCORE O.P. Eur J Prev Cardiol. 2016;23(10):1093-103. 
31. Verweij L, Peters RJG, op Reimer WJMS, Boekholdt MS, Luben RM, Wareham NJ, et al. Validation of the Systematic COronary Risk Evaluation - Older Persons (SCORE-OP) in the EPIC-Norfolk prospective population study. Int J Cardiol. 2019;293:226-30.

32. Rossello X, Dorresteijn JAN, Janssen A, Lambrinou E, Scherrenberg M, Bonnefoy-Cudraz E, et al. Risk prediction tools in cardiovascular disease prevention: A report from the ESC Prevention of CVD Programme led by the European Association of Preventive Cardiology (EAPC) in collaboration with the Acute Cardiovascular Care Association (ACCA) and the Association of Cardiovascular Nursing and Allied Professions (ACNAP). Eur J Prev Cardiol. 2019;2047487319846715.

33. Cook NR. Use and Misuse of the Receiver Operating Characteristic Curve in Risk Prediction. Circulation. 2007;115(7):928-35.

34. Timmis A, Rapsomaniki E, Chung SC, Pujades-Rodriguez M, Moayyeri A, Stogiannis D, et al. Prolonged dual antiplatelet therapy in stable coronary disease: comparative observational study of benefits and harms in unselected versus trial populations. BMJ. 2016;353:i3163.

35. Maasland L, van Oostenbrugge RJ, Franke CF, Scholte Op Reimer WJM, Koudstaal PJ, Dippel DWJ. Patients enrolled in large randomized clinical trials of antiplatelet treatment for prevention after transient ischemic attack or ischemic stroke are not representative of patients in clinical practice: the Netherlands Stroke Survey. Stroke. 2009;40(8):2662-8.

36. Warwick J, Falaschetti E, Rockwood K, Mitnitski A, Thijs L, Beckett N, et al. No evidence that frailty modifies the positive impact of antihypertensive treatment in very elderly people: an investigation of the impact of frailty upon treatment effect in the HYpertension in the Very Elderly Trial (HYVET) study, a double-blind, placebo-controlled study of antihypertensives in people with hypertension aged 80 and over. BMC Med. 2015;13(1):78.

37. Jaspers NEM, Blaha MJ, Matsushita K, van der Schouw YT, Wareham NJ, Khaw K-T, et al. Prediction of individualized lifetime benefit from cholesterol lowering, blood pressure lowering, antithrombotic therapy, and smoking cessation in apparently healthy people. Eur Heart J. 2019;31:1-10.

38. Kaasenbrood L, Bhatt DL, Dorresteijn JAN, Wilson PWF, D'Agostino RB, Massaro JM, et al. Estimated life expectancy without recurrent cardiovascular events in patients with vascular disease: The SMART-REACH model. J Am Heart Assoc. 2018;7(16).

39. Dorresteijn JAN, Kaasenbrood L, Cook NR, van Kruijsdijk RCM, van der Graaf Y, Visseren FLJ, et al. How to translate clinical trial results into gain in healthy life expectancy for individual patients. BMJ. 2016;352:11548. 


\section{Supplementary Material}

Supplemental Methods 1. Adding extra prognostic variables to the models

With the aim to improve the predictive value of the existing older person-specific score, we investigated the added value of adding optional information from additional risk factors based on previous literature. These additional risk factors included pulse pressure, ${ }^{1,2}$ ankle brachial index, ${ }^{3}$ frailty, ${ }^{4,5}$ presence of orthostatic hypotension, ${ }^{6}$ albuminuria, ${ }^{7,8}$ hsCRP, ${ }^{9-11}$ hs-CTnT, ${ }^{12,13} \mathrm{NT}$-proBNP, ${ }^{11}$ haematocrit, ${ }^{14}$ the presence of LVH on ECG, ${ }^{15,16}$ intima-media thickness (IMT), ${ }_{1}^{17}$ and pulse wave velocity (PWV). ${ }^{18}$ The final selection of variables was then made based on availability in the datasets. Only variables available in two or more of the datasets were chosen to enable external validation of the methodology. The definitions of the included variables can be found in Supplemental Table 2.

The additional determinants were incorporated in both model I and model II using the so-called naïve method. ${ }^{19,20}$ This method gives predictions based on the (population) baseline survival, median value of a variable in a population (for continuous variables), prevalence of a factor in the population (for categorical variables), and the independent hazard ratio of a variable. usina the followina formulas:

new individual risk $=1-(1-$ risk Elderly risk score $) \exp ^{\left.\operatorname{el} \log (H R) * \frac{\text { individual value variable }}{\text { median population value }}\right)}$ new individual risk $=1-(1-$ risk Elderly risk score $) \frac{H R}{\text { (prevalence factor } * H R)+(1-\text { prevalence) }}$ Hazard ratios and prevalence or population medians were derived in the MESA and/or ARIC study populations for all variables, depending on availability. The independent hazard ratios were derived using Fine and Gray non-CVD mortality competing risk adjusted models, adjusted for the variables from the older person-specific score. The hazard ratios are shown in Supplemental Table 3. After adding the additional determinants using the naïve method, model performance of the updated model (model III) was assessed with the c-statistic $(95 \% \mathrm{Cl})$ for discrimination and with calibration plots of predicted versus observed risk. The calibration plot can be found in Supplemental Figure 2. 
Supplemental Table 1. Individual risk prediction for major cardiovascular events (MACE; myocardial infarction, stroke, and CVD death)

\begin{tabular}{|c|c|c|}
\hline \multicolumn{3}{|c|}{ Risk estimation for patients with vascular disease } \\
\hline & 5-year: & 10-year: \\
\hline Scotland / Ireland & $\left(1-0.727^{\wedge} \exp (L P-2.389)\right) * 100 \%$ & $\left(1-0.529^{\wedge} \exp (L P-2.389)\right) * 100 \%$ \\
\hline Rest of North- and West-Europe: & $\left(1-0.828^{\wedge} \exp (L P-2.359)\right) * 100 \%$ & $\left(1-0.644^{\wedge} \exp (L P-2.359)\right) * 100 \%$ \\
\hline \multicolumn{3}{|c|}{ 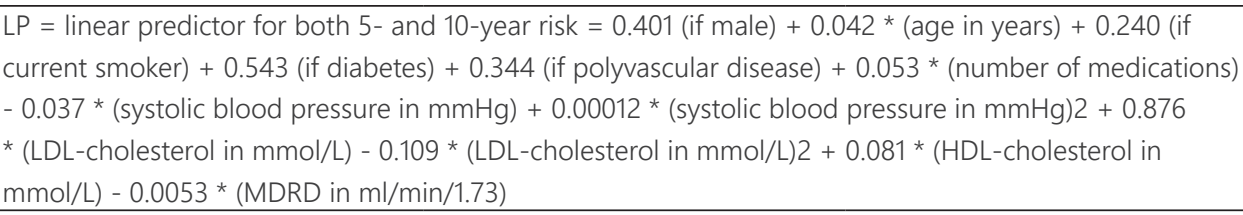 } \\
\hline \multicolumn{3}{|c|}{ Risk estimation for patients without vascular disease } \\
\hline & 5-year: & 10-year: \\
\hline Scotland / Ireland: & $\left(1-0.838^{\wedge} \exp (L P-1.934)\right) * 100 \%$ & $\left(1-0.703^{\wedge} \exp (L P-1.934)\right) * 100 \%$ \\
\hline Rest of North- and West-Europe: & $\left(1-0.895^{\wedge} \exp (L P-1.968)\right) * 100 \%$ & $\left(1-0.801^{\wedge} \exp (L P-1.968)\right) * 100 \%$ \\
\hline \multicolumn{3}{|c|}{$\begin{array}{l}\mathrm{LP}=\text { linear predictor for both } 5 \text { - and 10-year risk }=0.283 \text { (if male })+0.037 \text { * (age in years })+0.290 \\
\text { (if current smoker })+0.210 \text { (if diabetes })+0.090 * \text { (number of medications })+0.0060 * \text { (systolic blood } \\
\text { pressure in } \mathrm{mmHg})+0.0070 *(\mathrm{LDL} \text {-cholesterol in } \mathrm{mmol} / \mathrm{L})-0.359 *(\mathrm{HDL} \text {-cholesterol in mmol/L) }-0.061 \\
*(\mathrm{MDRD} \text { in } \mathrm{ml} / \mathrm{min} / 1.73)+0.00048 *(\mathrm{MDRD} \text { in } \mathrm{ml} / \mathrm{min} / 1.73) 2\end{array}$} \\
\hline
\end{tabular}

\section{Individual risk prediction for MACE plus heart failure (MACE-HF)}

For patients with vascular disease: add 0.362 to the linear predictor in the formula above.

For patients without vascular disease: add 0.180 to the linear predictor

\section{Using the model to calculate treatment effects}

\section{Statin treatment}

For patients with vascular disease: add -0.245 to the linear predictor.

For patients without vascular disease: add -0.140 to the linear predictor

Absolute risk reduction (ARR) = individual MACE risk (\%) without a statin - individual MACE risk (\%) with a statin

\section{Blood pressure lowering}

Add -0.0223 * every mmHg expected systolic blood pressure decrease (to reach the target systolic blood pressure) to the linear predictor.

ARR = individual MACE risk (\%) without blood pressure lowering - individual MACE risk (\%) with blood pressure lowering to target systolic blood pressure. 


\section{Supplemental Table 2. Definitions of variables used in the study}

\begin{tabular}{|c|c|c|}
\hline Variable & $\begin{array}{l}\text { Included in } \\
\text { which studies? }\end{array}$ & Definition \\
\hline Frailty & ARIC, HYVET & $\begin{array}{l}\text { Patients are categorized as robust, pre-frail, or frail according to pre- } \\
\text { specified cut-off values, specific for the frailty index used. } \\
\text { In ARIC: } 0 \text { items; } 1 \text { or } 2 \text { items; }>3 \text { items of the following } 5 \\
\text { components: weight loss, exhaustion, low energy expenditure, } \\
\text { slowness, weakness (21) } \\
\text { In HYVET: }<0.10 ; 0.10-0.35 ;>0.35 \text { score based on } 60 \text { deficits (22) }\end{array}$ \\
\hline Pulse pressure & $\begin{array}{l}\text { ARIC, HYVET, } \\
\text { MESA, SMART, } \\
\text { SPRINT }\end{array}$ & Systolic blood pressure minus diastolic blood pressure (in $\mathrm{mmHg}$ ) \\
\hline $\begin{array}{l}\text { Ankle brachial } \\
\text { index }\end{array}$ & $\begin{array}{l}\text { ARIC, MESA, } \\
\text { SMART }\end{array}$ & $\begin{array}{l}\text { The ratio between the highest systolic blood pressure measured in } \\
\text { each ankle (posterior tibia and dorsal pedal arteries) and the highest } \\
\text { blood pressure in both brachial arteries. Patients are categorized } \\
\text { as having an ankle-brachial index }<0.90,0.90-1.30,>1.30 \text {, using the } \\
\text { lowest of the two measurements }\end{array}$ \\
\hline Albuminuria & $\begin{array}{l}\text { ARIC, MESA, } \\
\text { SMART, SPRINT }\end{array}$ & $\begin{array}{l}\text { Categorized using KDIGO classification: A1 (urinary albumin/ } \\
\text { creatinine ratio <30 mg/g), A2 (30-299 mg/g), A3 (> } 300 \text { mg/g) }\end{array}$ \\
\hline $\begin{array}{l}\text { High sensitivity- } \\
\text { CRP }\end{array}$ & $\begin{array}{l}\text { ARIC, MESA, } \\
\text { SMART }\end{array}$ & Measured in $\mathrm{mmol} / \mathrm{L}$ \\
\hline Hematocrit & $\begin{array}{l}\text { ARIC, HYVET, } \\
\text { SMART }\end{array}$ & Plasma measurement, in \% \\
\hline $\begin{array}{l}\text { Left ventricular } \\
\text { hypertrophy (LVH) } \\
\text { on ECG }\end{array}$ & $\begin{array}{l}\text { ARIC, HYVET, } \\
\text { MESA }\end{array}$ & LVH Present By Cornell Definition (yes/no) \\
\hline $\begin{array}{l}\text { Intima media } \\
\text { thickness (IMT) }\end{array}$ & SMART, MESA & $\begin{array}{l}\text { Mean common carotid IMT measured using Doppler-assisted duplex } \\
\text { scanning, in millimeters }\end{array}$ \\
\hline
\end{tabular}


Supplemental Table 3: Independent hazard ratios (HR) with $95 \%$ confidence intervals (CI) for optional prognostic variables.

\begin{tabular}{lll}
\hline & MACE & MACE-HF \\
\cline { 2 - 3 } High-sensitivity C-reactive protein (per ng/L) & $1.02(1.01-1.02)$ & $1.02(1.01-1.03)$ \\
Frailty & & \\
Robust & $1($ ref $)$ & 1 (ref) \\
Pre-frail & $1.31(1.11-1.54)$ & $1.31(1.13-1.52)$ \\
Frail & $1.83(1.41-2.38)$ & $1.74(1.37-2.20)$ \\
Ankle brachial index (ABI) & & \\
ABI 0.9-1.3 & $1($ ref $)$ & $1($ ref $)$ \\
ABI < 0.9 & $1.46(1.26-1.68)$ & $1.36(1.18-1.56)$ \\
ABI >1.3 & $0.85(0.63-1.15)$ & $0.97(0.74-1.26)$ \\
Hematocrit (per \%) & $0.99(0.97-1.01)$ & $0.98(0.96-0.99)$ \\
IMT (per mm) & $1.52(1.11-2.07)$ & $1.49(1.09-2.04)$ \\
Left ventricular hypertrophy on ECG & $1.31(1.04-1.64)$ & $1.51(1.24-1.85)$ \\
Albuminuria & & 1 (ref) \\
Urinary ACR <30 mg/g & $1($ ref $)$ & $1.31(1.17-1.47)$ \\
Urinary ACR 30-300 mg/g & $1.24(1.09-1.40)$ & $1.32(1.04-1.67)$ \\
Urinary ACR >300 mg/g & $1.21(0.94-1.57)$ & $1.02(0.97-1.08)$ \\
Pulse pressure (per 10 mmHg) & $0.99(0.93-1.05)$ & \\
\hline
\end{tabular}


Supplemental Figure 1. Calibration of the Elderly risk score in subgroups based on age, history of cardiovascular disease, and sex

(A) For the prediction of MACE
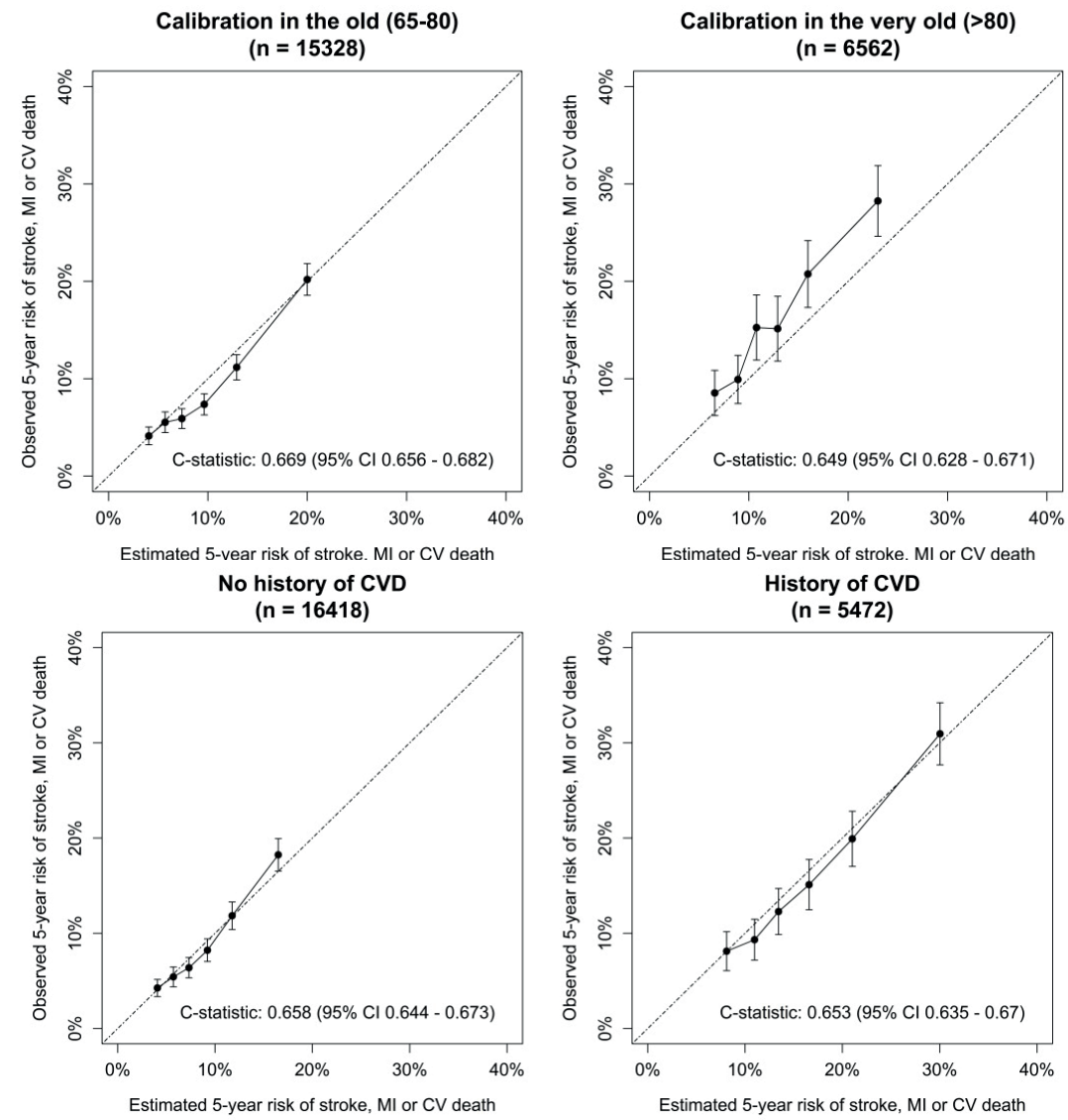

Calibration in women
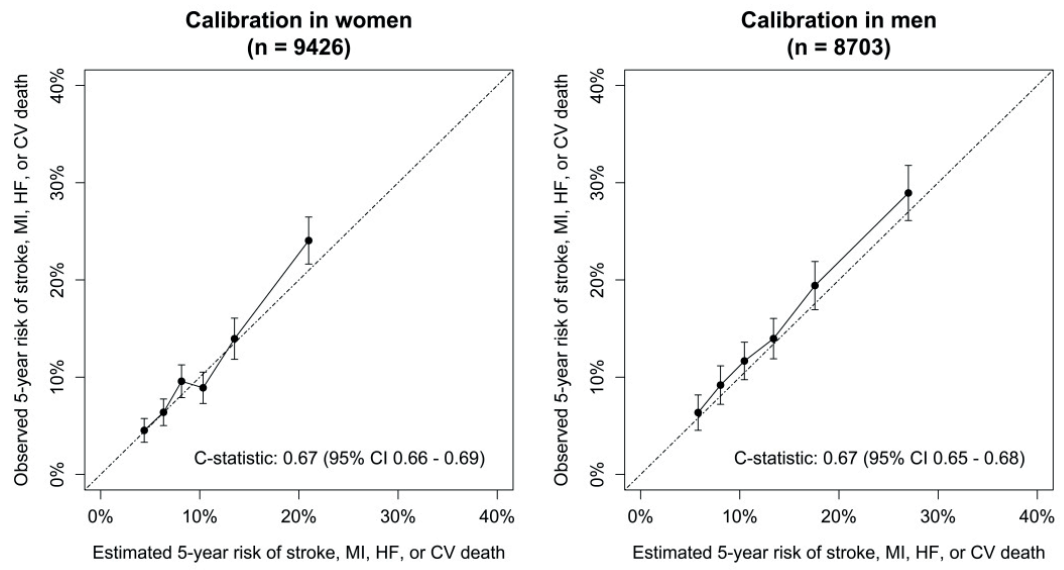
(B) For the prediction of MACE-HF
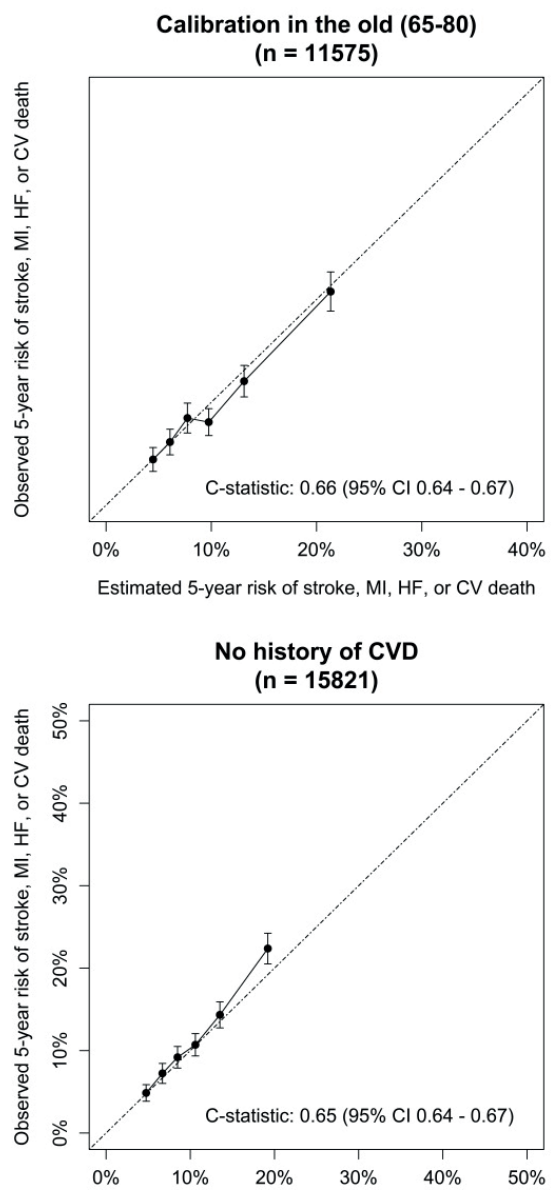

Estimated 5-year risk of stroke, MI, HF, or CV death

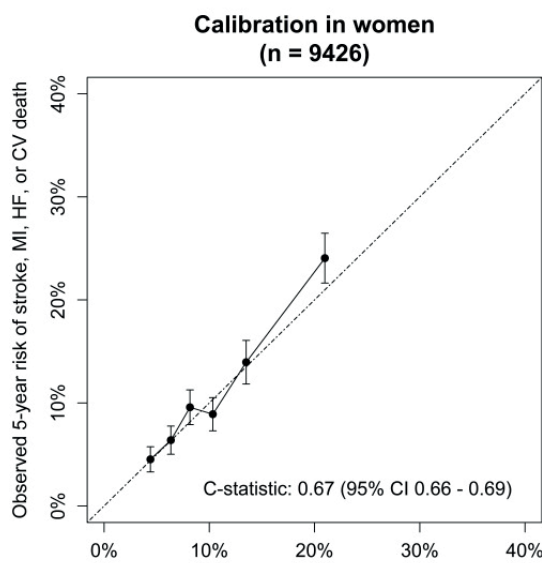

Estimated 5-year risk of stroke, MI, HF, or CV death
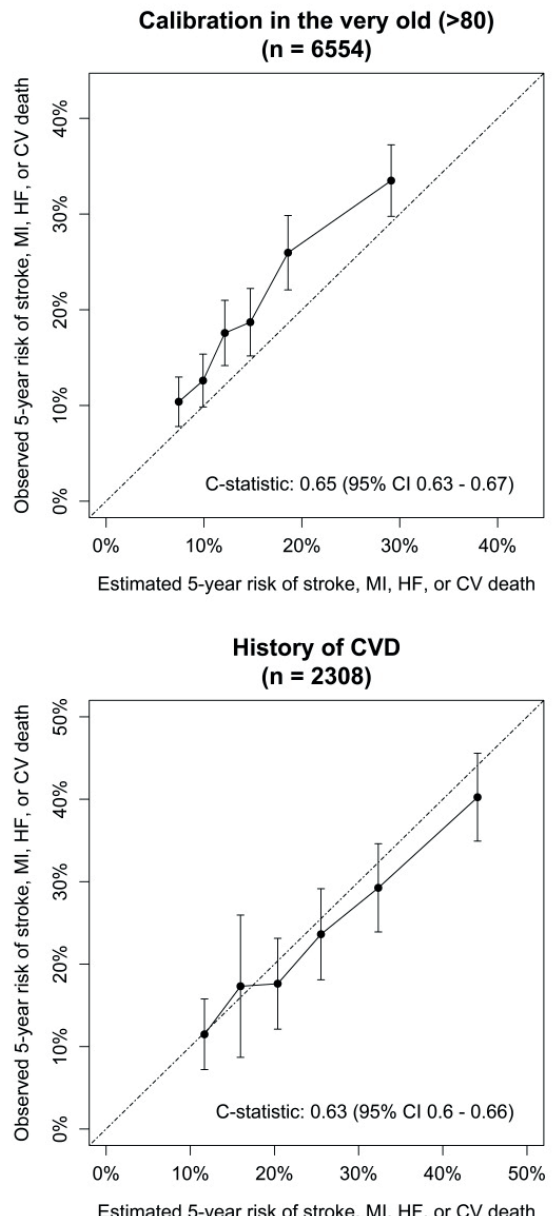

Estimated 5-year risk of stroke, MI, HF, or CV death

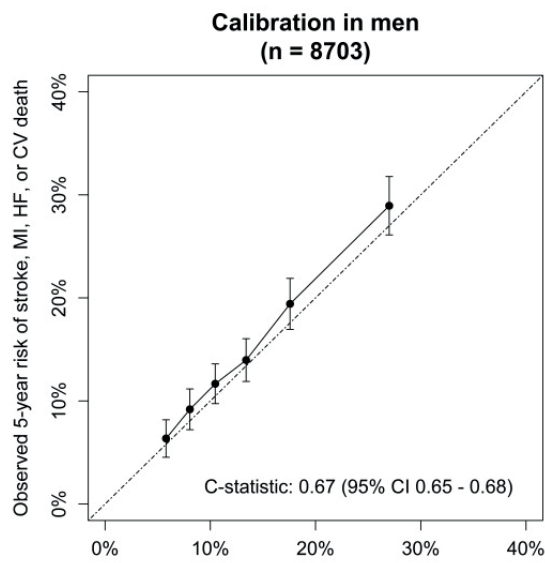

Estimated 5-year risk of stroke, MI, HF, or CV death 
Supplementary Figure 2. Validation of the Elderly risk model including additional prognostic variables for the prediction of the risk of (A) major cardiovascular events (MACE), and (B) MACE plus heart failure

(A) Naive method for prediction of MACE ( $n=21890)$

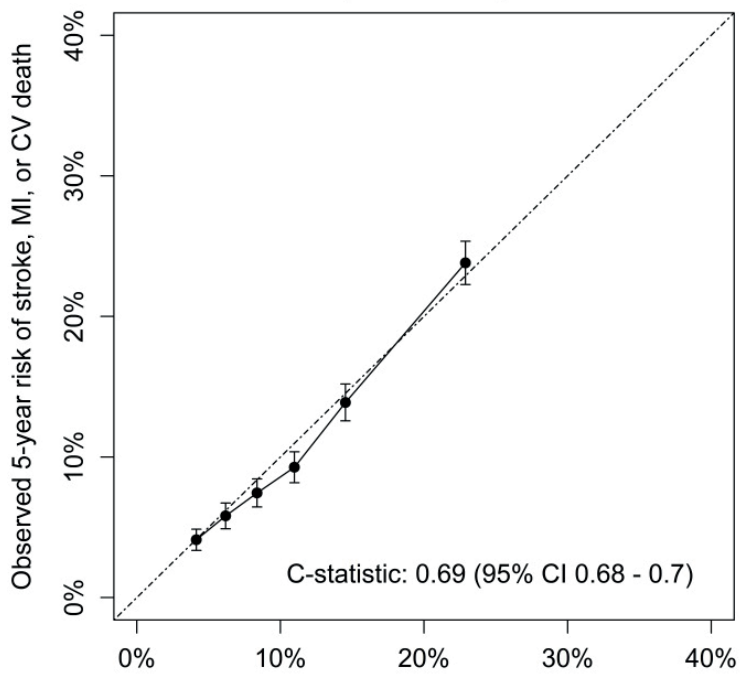

Estimated 5-year risk of stroke, MI, or CV death

(B) Naive method for prediction of MACE-HF ( $n=19279$ )

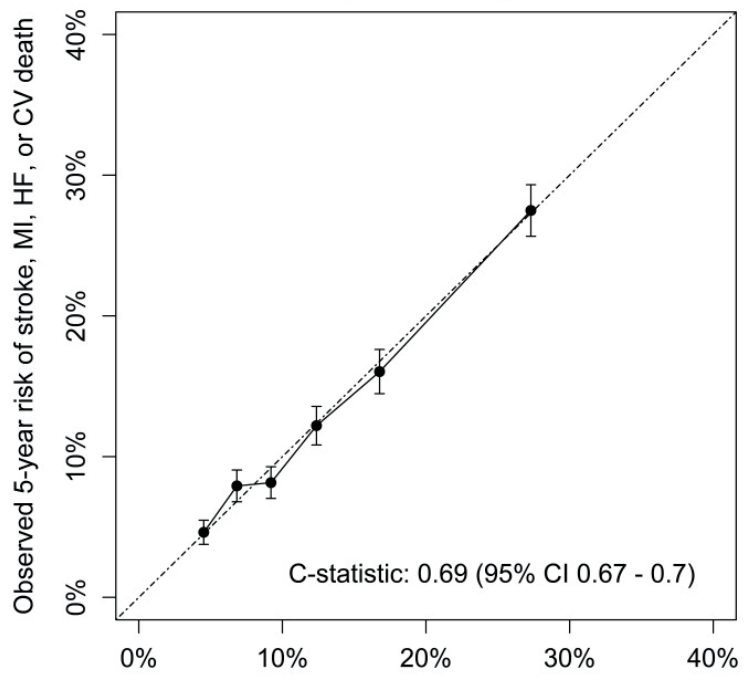

Estimated 5-year risk of stroke, MI, HF, or CV death 
Supplemental Figure 3. Older person-specific score charts for the prediction of 10-year risk of MACE

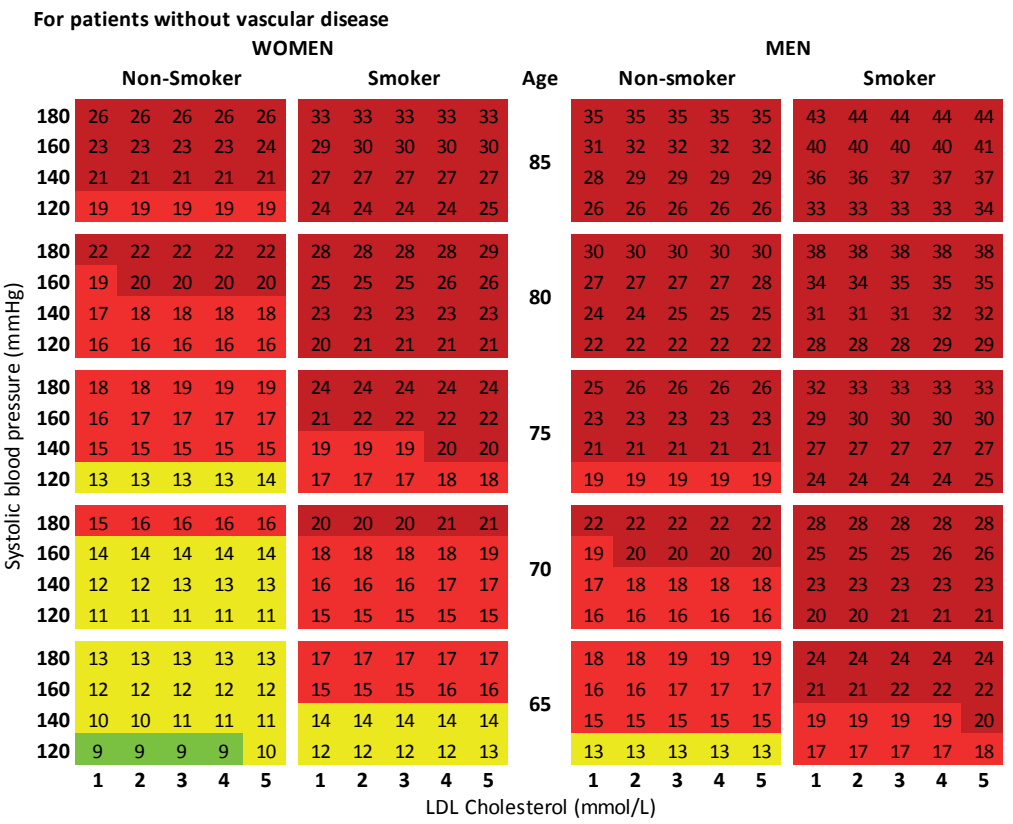

For patients with vascular disease

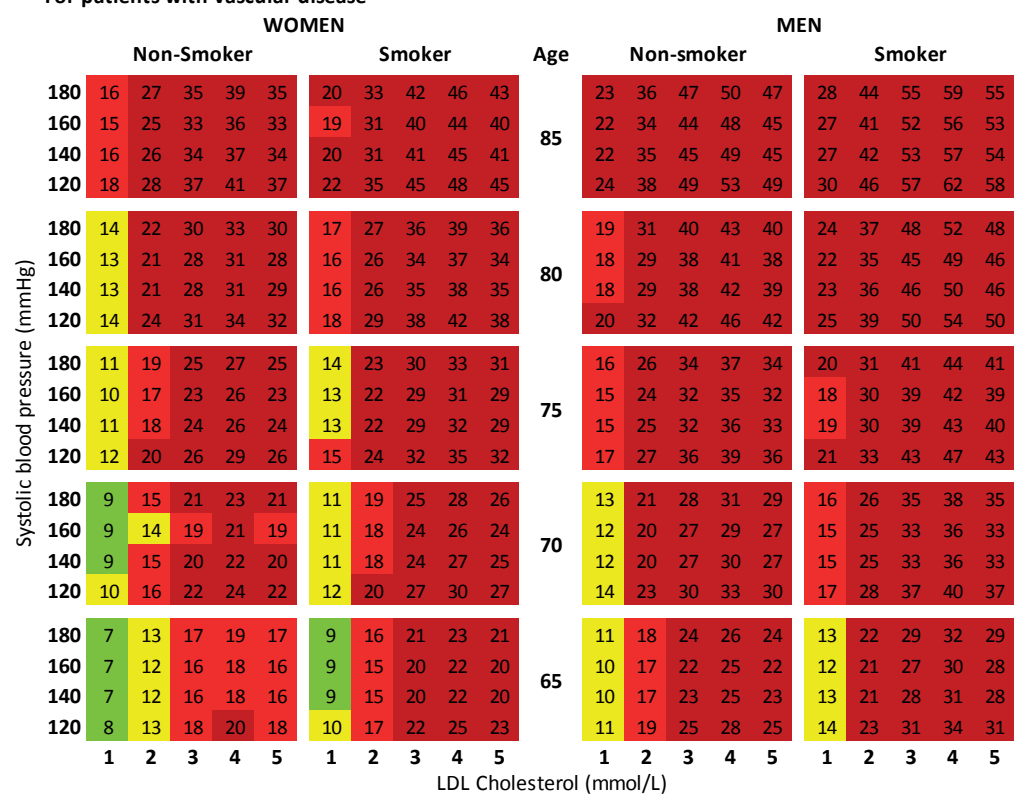

Assumes HDL-cholesterol of $1.2 \mathrm{mmol} / \mathrm{L}$ in men and $1.4 \mathrm{mmol} / \mathrm{L}$ in women; no diabetes mellitus; 5 different medications used; an eGFR of $70 \mathrm{ml} / \mathrm{min} / 1.73$; and (in case of a history of cardiovascular disease) no polyvascular disease. The figures in the cells indicate the \% 10-year risk of total CVD events (MACE; myocardial infarction, stroke, CV mortality). For patients with diabetes mellitus or polyvascular disease; multiply risk by 1.5 . 
Supplementary Figure 4. Histograms showing the 5-year risk of (A) major cardiovascular events (MACE; myocardial infarction, stroke, or cardiovascular death), and (B) MACE plus heart failure (MACE-HF) in older patients without clinically manifest vascular disease at baseline; and the risk of (C) MACE, and (D) MACE-HF in older patients with clinically manifest vascular disease at baseline.
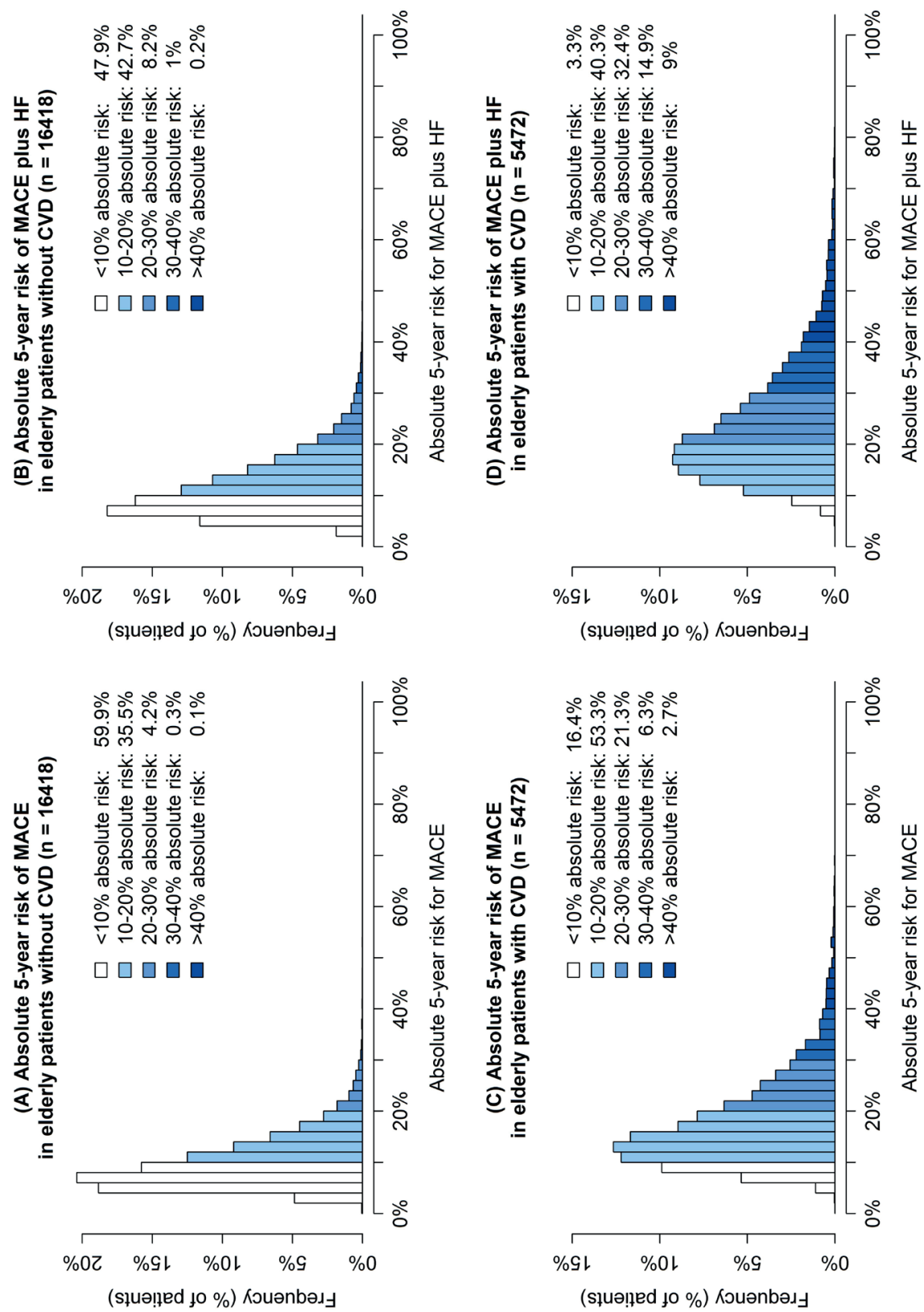
Supplementary Figure 5. Histograms showing the 5-year absolute risk reduction from bloodpressure lowering in older patients with an indication for blood-pressure lowering therapy for (A) major cardiovascular events (MACE; myocardial infarction, stroke, or cardiovascular death), and (B) MACE plus heart failure (MACE-HF) in patients without clinically manifest vascular disease at baseline; and for (C) MACE, and (D) MACE-HF in patients with clinically manifest vascular disease at baseline.
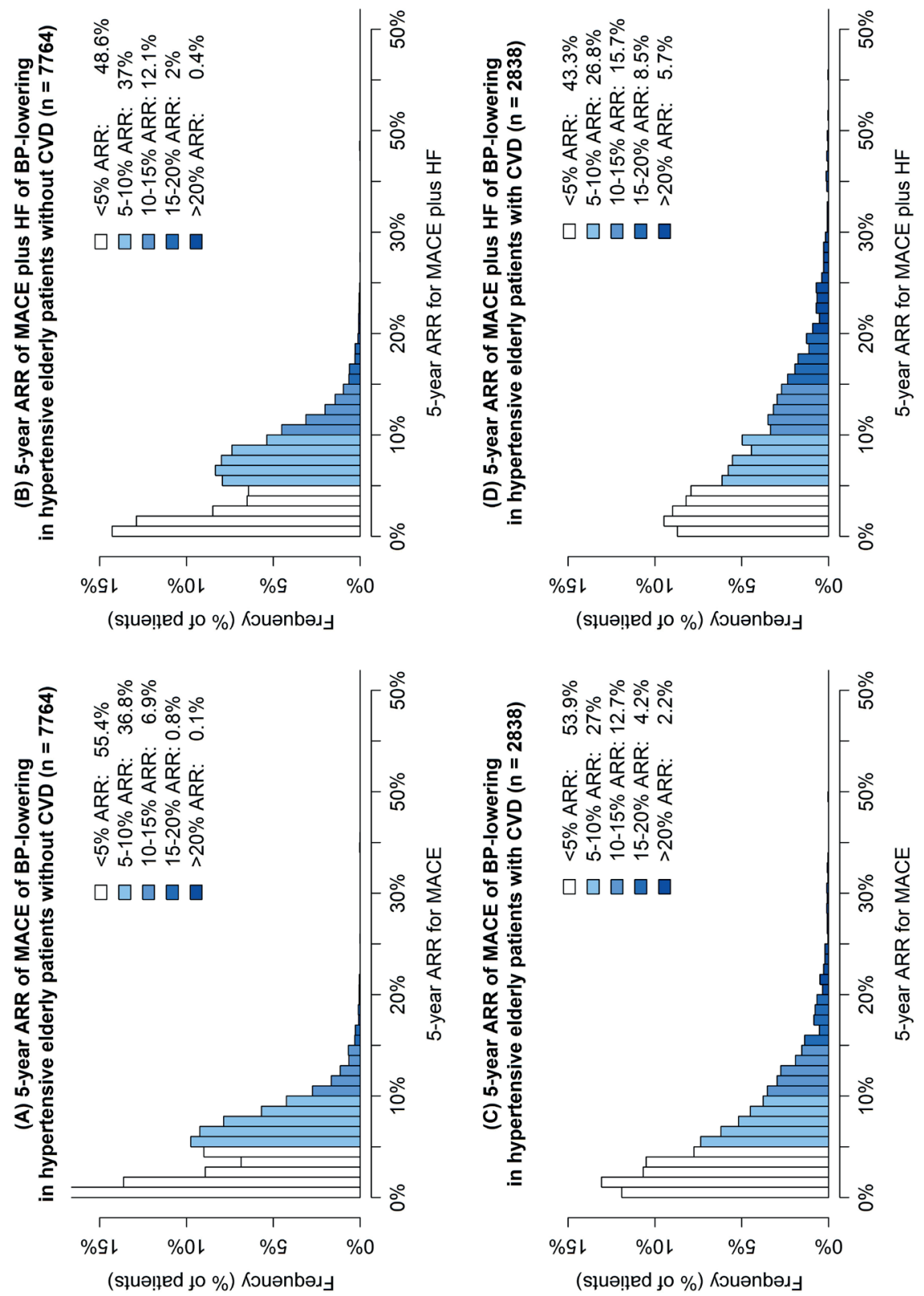
Supplementary Figure 6. Sensitivity analyses for the individualized estimations of absolute risk reduction from blood-pressure lowering in older patients: $(A)$ in men; $(B)$ in women; and $(C)$ in patients using lipid-lowering at baseline.
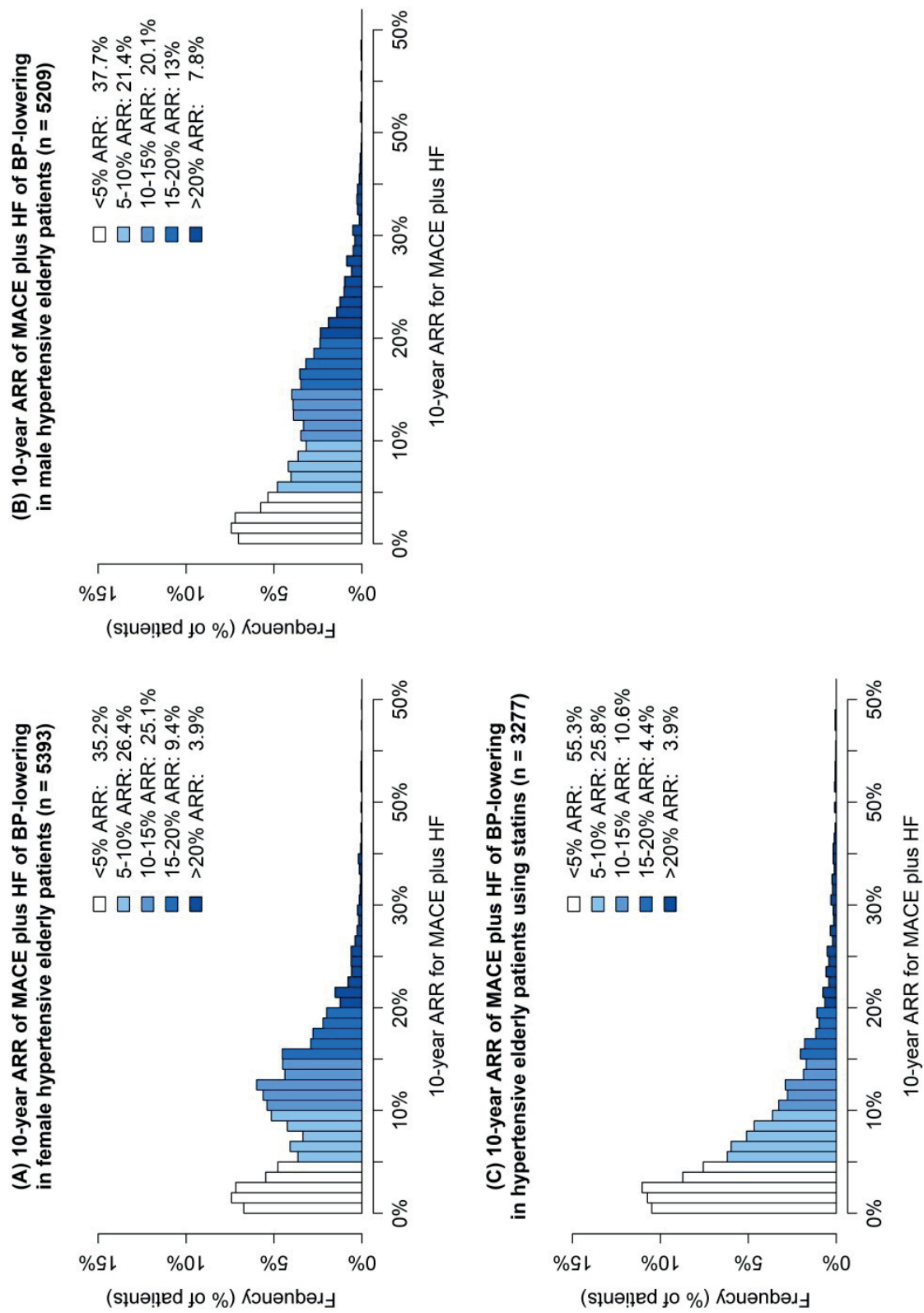

In women, the median ARR for MACE-HF was 8.2\% (IQR 3.1-12.2), while in men, the median ARR was $7.7 \%$ (IQR 2.9-13.8). In patients using lipid-lowering therapy at baseline, the median ARR for MACE-HF was $4.4 \%$ (IQR 2-8.4). 


\section{References used in Supplementary Material}

1. Vaccarino V, Holford TR, Krumholz HM. Pulse pressure and risk for myocardial infarction and heart failure in the elderly. J Am Coll Cardiol. 2000;36(1):130-8.

2. Vaccarino V, Berger AK, Abramson J, Black HR, Setaro JF, Davey JA, et al. Pulse pressure and risk of cardiovascular events in the systolic hypertension in the elderly program. Am J Cardiol. 2001;88(9):980-6.

3. Collaboration ABI, Fowkes FGR, Murray GD, Butcher I, Heald CL, Lee RJ, et al. Ankle brachial index combined with Framingham Risk Score to predict cardiovascular events and mortality: a metaanalysis. JAMA. 2008 Jul;300(2):197-208.

4. Veronese N, Cereda E, Stubbs B, Solmi M, Luchini C, Manzato E, et al. Risk of cardiovascular disease morbidity and mortality in frail and pre-frail older adults: Results from a meta-analysis and exploratory meta-regression analysis. Ageing Res Rev. 2017;35:63-73.

5. Khan H, Kalogeropoulos AP, Georgiopoulou V V, Newman AB, Harris TB, Rodondi N, et al. Frailty and risk for heart failure in older adults: the health, aging, and body composition study. Am Heart J. 2013;166(5):887-94.

6. Xin W, Mi S, Lin Z, Wang H, Wei W. Orthostatic hypotension and the risk of incidental cardiovascular diseases: A meta-analysis of prospective cohort studies. Prev Med (Baltim). 2016;85:90-7.

7. Xia F, Liu G, Shi Y, Zhang Y. Impact of microalbuminuria on incident coronary heart disease, cardiovascular and all-cause mortality: a meta-analysis of prospective studies. Int J Clin Exp Med. 2015;8(1):1-9.

8. Perkovic V, Verdon C, Ninomiya T, Barzi F, Cass A, Patel A, et al. The relationship between proteinuria and coronary risk: a systematic review and meta-analysis. PLoS Med. 2008;5(10):e207-e207.

9. Cushman M, Arnold AM, Psaty BM, Manolio TA, Kuller LH, Burke GL, et al. C-reactive protein and the 10-year incidence of coronary heart disease in older men and women: the cardiovascular health study. Circulation. 2005;112(1):25-31.

10. Tracy RP, Lemaitre RN, Psaty BM, Ives DG, Evans RW, Cushman M, et al. Relationship of C-reactive protein to risk of cardiovascular disease in the elderly. Results from the Cardiovascular Health Study and the Rural Health Promotion Project. Arterioscler Thromb Vasc Biol. 1997;17(6):1121-7.

11. van Peet PG, Drewes YM, de Craen AJM, Gussekloo J, de Ruijter W. NT-proBNP best predictor of cardiovascular events and cardiovascular mortality in secondary prevention in very old age: the Leiden 85-plus Study. PLoS One. 2013;8(11):e81400-e81400.

12. Jørgen G, T. AE, H. NS, Thor U, John W, J.V. MJ, et al. Prognostic Effect of High-Sensitive Troponin T Assessment in Elderly Patients With Chronic Heart Failure. Circ Hear Fail. 2014;7(1):96-103.

13. Wu W, Li D-X, Wang $Q, X u Y$, Cui Y-J. Relationship between high-sensitivity cardiac troponin T and the prognosis of elderly inpatients with non-acute coronary syndromes. Clin Interv Aging. 2018;13:1091-8.

14. Gagnon DR, Zhang TJ, Brand FN, Kannel WB. Hematocrit and the risk of cardiovascular disease--the Framingham study: a 34-year follow-up. Am Heart J. 1994 Mar;127(3):674-82.

15. Antikainen RL, Peters R, Beckett NS, Fagard RH, Wang J-G, Rajkumar C, et al. Left ventricular 
hypertrophy is a predictor of cardiovascular events in elderly hypertensive patients: Hypertension in the Very Elderly Trial. J Hypertens. 2016;34(11):2280-6.

16. van Kleef MEAM, Visseren FLJ, Vernooij JWP, Nathoe HM, Cramer M-JM, Bemelmans RHH, et al. Four ECG left ventricular hypertrophy criteria and the risk of cardiovascular events and mortality in patients with vascular disease. J Hypertens. 2018 Sep;36(9):1865-73.

17. van den Oord SCH, Sijbrands EJG, ten Kate GL, van Klaveren D, van Domburg RT, van der Steen AFW, et al. Carotid intima-media thickness for cardiovascular risk assessment: systematic review and meta-analysis. Atherosclerosis. 2013 May;228(1):1-11.

18. Zhong Q, Hu M-J, Cui Y-J, Liang L, Zhou M-M, Yang Y-W, et al. Carotid-Femoral Pulse Wave Velocity in the Prediction of Cardiovascular Events and Mortality: An Updated Systematic Review and MetaAnalysis. Angiology. 2018 Aug;69(7):617-29.

19. Tyrer J, Duffy SW, Cuzick J. A breast cancer prediction model incorporating familial and personal risk factors. Stat Med. 2004;23(7):1111-30.

20. Berkelmans GFN, Read SH, Gudbjörnsdottir S, Wild SH, Franzen S, Svensson A-M, et al. Lifetime predictions for individualized vascular disease prevention [thesis]. Utrecht (the Netherlands); 2018. Ch. 5: Dealing with missing patient characteristics when using cardiovascular prediction models in clinical practice; p. 109.

21. Kucharska-Newton AM, Palta P, Burgard S, Griswold ME, Lund JL, Capistrant BD, et al. Operationalizing Frailty in the Atherosclerosis Risk in Communities Study Cohort. J Gerontol A Biol Sci Med Sci. 2017;72(3):382-8.

22. Warwick J, Falaschetti E, Rockwood K, Mitnitski A, Thijs L, Beckett N, et al. No evidence that frailty modifies the positive impact of antihypertensive treatment in very elderly people: an investigation of the impact of frailty upon treatment effect in the HYpertension in the Very Elderly Trial (HYVET) study, a double-blind, placeb. BMC Med. 2015;13(1):78. 


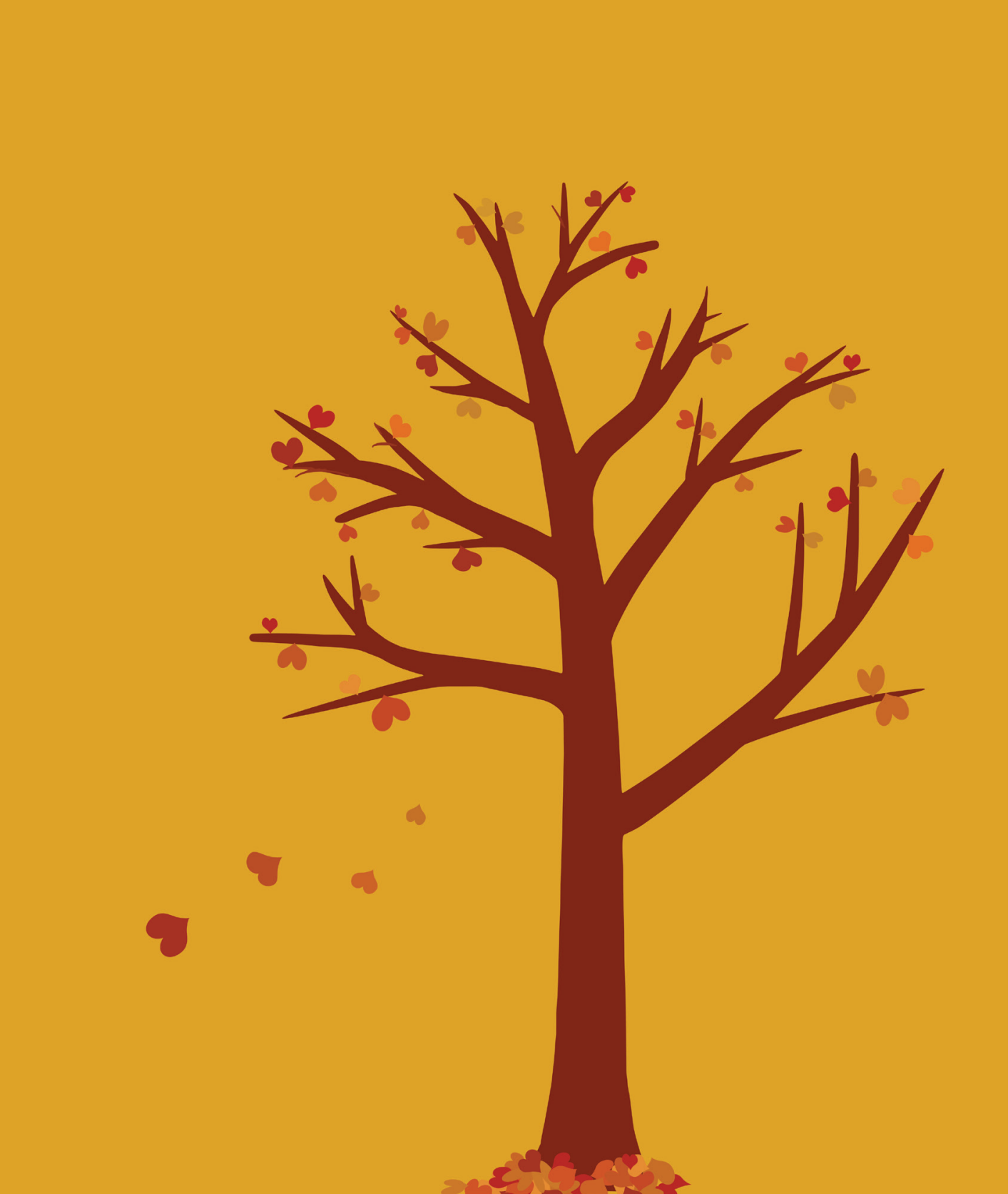


Lifetime benefit of cholesterol-lowering, blood pressure-lowering, and smoking cessation in people without cardiovascular disease: a changing paradigm in cardiovascular disease prevention

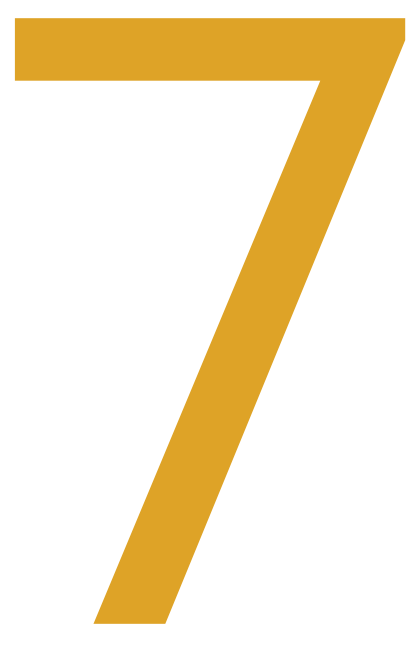

\author{
Tamar I. de Vries \\ Steven H.J. Hageman \\ Jan Westerink \\ Frank L.J. Visseren
} Manuscript draft. 


\section{ABSTRACT}

Treatment decisions for cholesterol-lowering and blood pressure-lowering for apparently healthy persons are usually based on 10-year CVD risk estimations. There are inherent difficulties with this approach as young persons always have a low 10-year CVD risk even in the presence of high risk factor levels, and older persons always have a high 10-year CVD risk but may expect limited benefit from risk factor treatment. The purpose of this paper is to explore how lifetime estimations can be used as an alternative method for medical decision making, by providing lifetime estimates of CVD risk and lifetime benefit from cholesterol-lowering, blood pressure-lowering and smoking cessation in apparently healthy persons.

Treatment effects from preventive therapy can be estimated by combining multivariate risk predictions with relative treatment effects from meta-analyses. In this paper, lifetime treatment effects were estimated of cholesterol-lowering, blood pressure-lowering and smoking cessation, expressed as "years without CVD gained" using the competing riskadjusted LIFEtime-perspective CardioVascular Disease (LIFE-CVD) model. Using a lifetime CVD risk and benefit approach it becomes apparent that the lifetime benefit of cholesterollowering or blood-pressure lowering diminishes with increasing age. Smoking cessation is beneficial at all ages, although the effects are largest in youngest subjects. Non-smokers have the largest lifetime benefit of cholesterol-lowering and blood pressure-lowering. Estimation of lifetime CVD risk and benefit can be used in discussions between health care professional and patient and may guide treatment decisions in clinical practice. 


\section{Background}

In daily clinical practice, clinicians routinely make, together with patients, treatment decisions for cardiovascular risk management for individual patients. In the primary prevention of cardiovascular disease (CVD), current international guidelines advise lowering of blood pressure and low-density lipoprotein (LDL)-cholesterol based on threshold levels in combination with an individual estimated 10-year CVD risk above a certain level, for example a 10-year CVD mortality risk of $>5$ or $>10 \%{ }^{1,2}$ Several riskassessment tools have been developed to estimate 10-year risk of cardiovascular events or mortality in the primary prevention setting, including Systematic COronary Risk Evaluation (SCORE), which is recommended for use in primary prevention by the European Society of Cardiology (ESC) 2016 guidelines on the prevention of cardiovascular disease, and the ASCVD score used in North-America. ${ }^{3-5}$ However, medical decision strategies based on $10-$ year risk stratification have several inherent limitations.

The purpose of this paper is to explain the limitations of this risk-based approach, and to explain how estimations of lifetime risk and lifetime treatment effects can be used in clinical practice as an additional or alternative approach in CVD prevention decision making. Lifetime benefit tables are presented for cholesterol-lowering, blood pressurelowering and smoking cessation in persons without manifest cardiovascular disease.

\section{Using 10-year risk predictions in clinical practice}

Treatment decisions in current international CVD prevention guidelines are largely based on estimated CV. Patients at highest risk of future events, based on either their medical history or predicted risk using risk scores, are recommended to start cholesterol-lowering and/or blood pressure-lowering and to reach treatment targets for LDL-cholesterol and systolic blood pressure. ${ }^{1,2}$

The 2016 ESC guideline on CVD prevention presents risk charts based on the SCORE risk model. The following risk categories are then defined based on the estimated 10-year risk: low-risk (<1\% 10-year risk), moderate-risk (1-5\%), high-risk (5-10\%), and very high-risk $(\geq 10 \%)$. These risk categories correspond to treatment recommendations. For example, pharmacological lipid-lowering is advised for all persons with an LDL-cholesterol $\geq 1.8$ $\mathrm{mmol} / \mathrm{L}$ at very high-risk of CVD, and for all persons with an LDL-cholesterol $\geq 2.6 \mathrm{mmol} / \mathrm{L}$ at high risk of CVD. ${ }^{1}$ Similarly, for blood pressure, the general treatment target is an SBP < $140 \mathrm{mmHg}$, but a target SBP $<120 \mathrm{mmHg}$ can be considered for patients at (very) highrisk for CVD who can tolerate multiple BP lowering drugs. ${ }^{1}$ 
Figure 1a shows a risk chart of 10-year fatal CVD risk for patients with different risk profiles as estimated with the SCORE risk model (until age 65) and an older person-specific, competing risk adjusted, risk model as derived in the PROSPER trial and externally validated (from age 70). ${ }^{6}$ Details on the methodology used can be found in Supplementary Methods 1.

Figure 1. Two-dimensional chart of (A) 10-year risk of fatal CVD; (B) lifetime risk of major cardiovascular events (MACE).

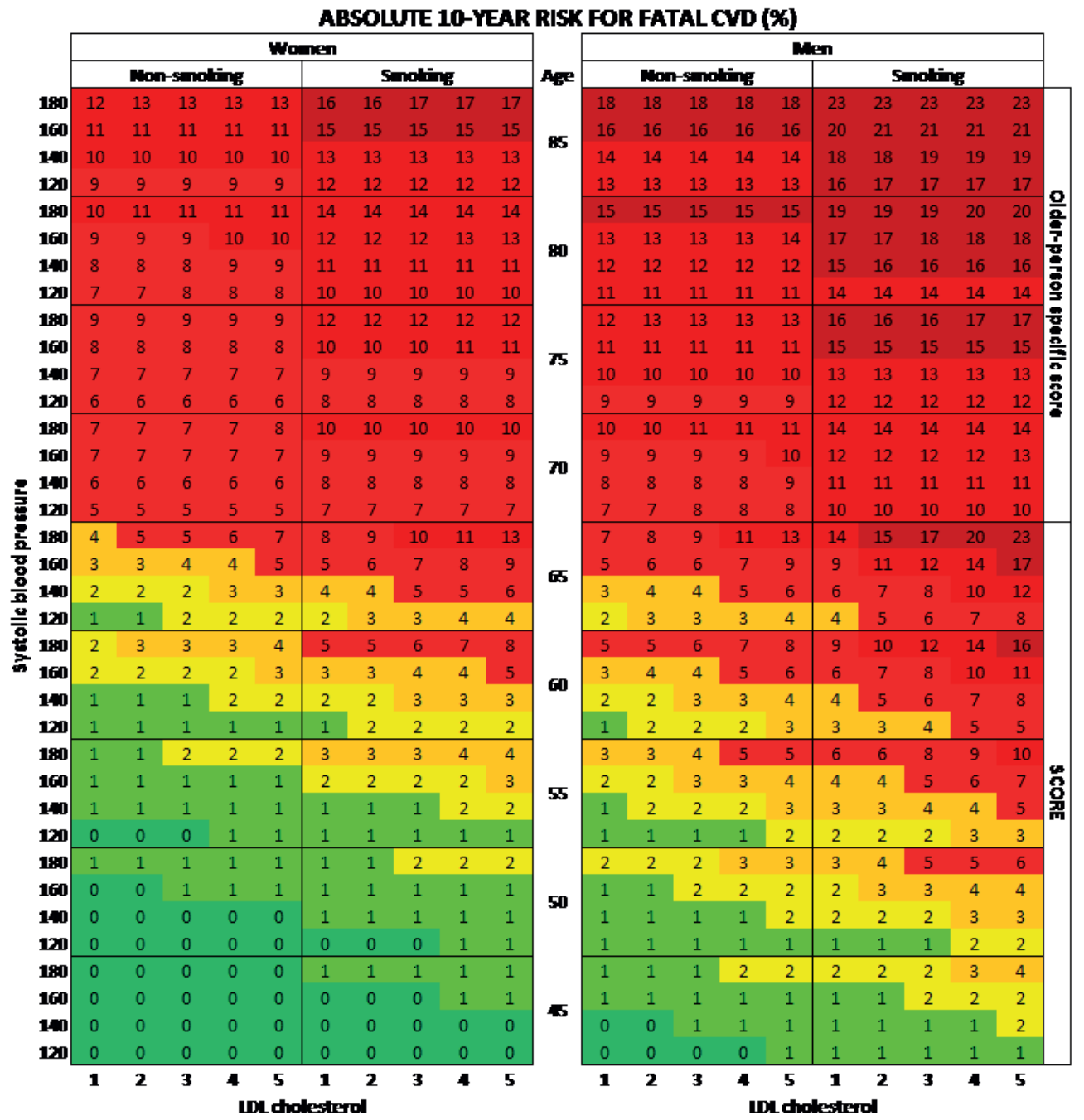

The following population median values were used: BMI of $26 \mathrm{~kg} / \mathrm{m} 2$, no diabetes, no parental history of $\mathrm{MI}$, non-HDL-cholesterol of $0.8 \mathrm{mmol} / \mathrm{L}$ higher than $\mathrm{LDL}$-cholesterol, and HDL of $1.2 \mathrm{mmol} / \mathrm{L}$ for men and $1.4 \mathrm{mmol} / \mathrm{L}$ for women, and in patients aged over 69 years, 5 medications used, and estimated glomerular filtration rate of $67 \mathrm{ml} / \mathrm{min} / 1.73 \mathrm{~m} 2$ for women and $70 \mathrm{ml} / \mathrm{min} / 1.73 \mathrm{~m} 2$ for men 
Figure 1. (continued)

UFETIME RISK FOR MACE (\%)

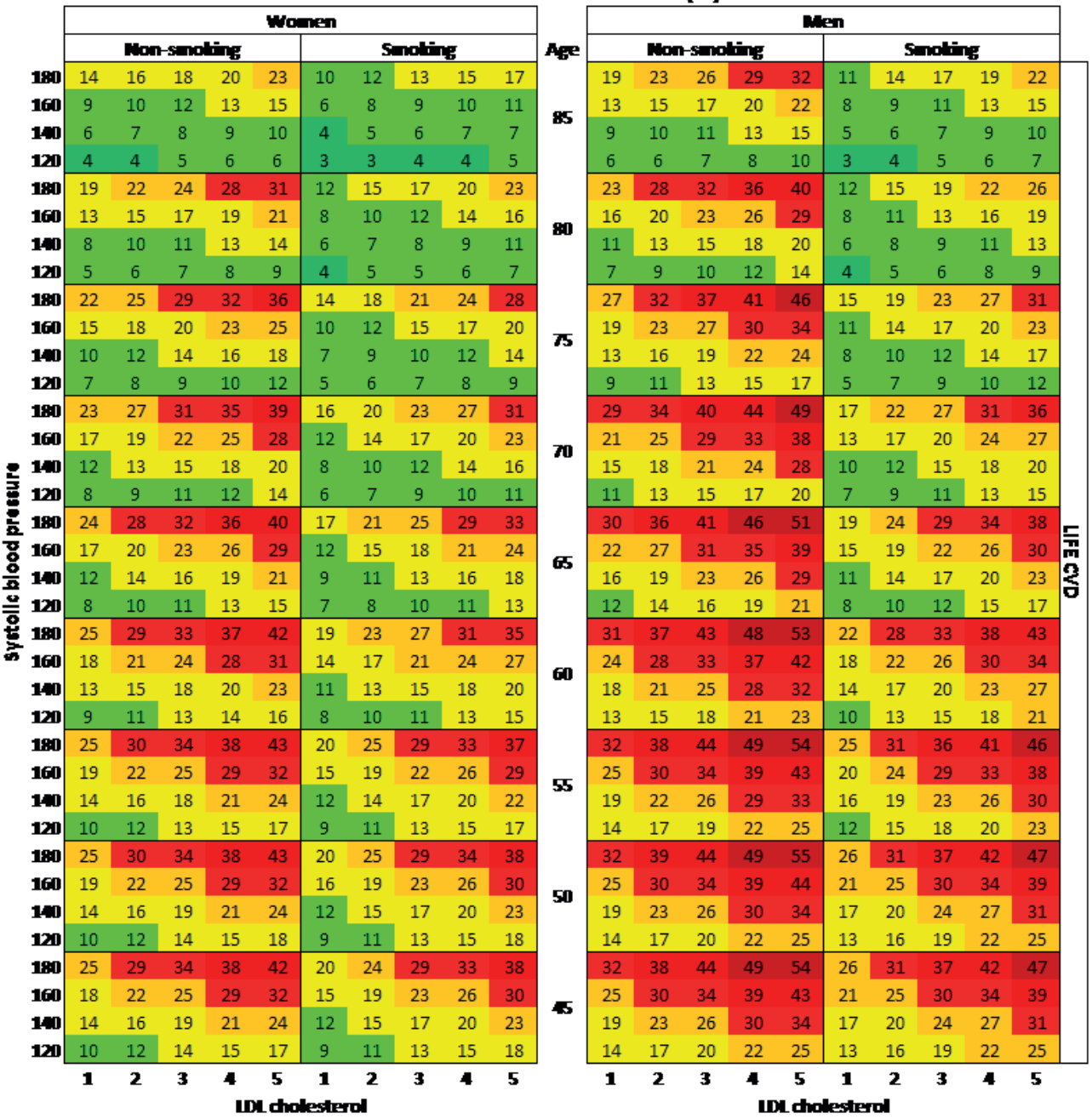

The following population median values were used: BMI of $26 \mathrm{~kg} / \mathrm{m} 2$, no diabetes, no parental history of MI, non-HDL-cholesterol of $0.8 \mathrm{mmol} / \mathrm{L}$ higher than $L D L$-cholesterol, and HDL of $1.2 \mathrm{mmol} / \mathrm{L}$ for men and $1.4 \mathrm{mmol} / \mathrm{L}$ for women, and in patients aged over 69 years, 5 medications used, and estimated glomerular filtration rate of $67 \mathrm{ml} / \mathrm{min} / 1.73 \mathrm{~m} 2$ for women and $70 \mathrm{ml} / \mathrm{min} / 1.73 \mathrm{~m} 2$ for men 


\section{The uses and limitations of 10 -year risk models}

Figure 1a immediately shows how 10-year risks can but also cannot be used for a personalized approach. In the age range between 55 to 65 years, the SCORE chart helps in distinguishing between patients with low-risk and patients with very high-risk. Under the assumption that very high-risk patients will have the largest benefit from preventive strategies, in this age range SCORE helps to discriminate between patients who will benefit less or more from treatment of cardiovascular risk factors, allowing an individualized approach. However, in younger subjects (age <55 years) almost all patients are at low or moderate-risk even in the presence of high cholesterol, elevated blood pressure and even smoking. Similarly, in the higher age range ( $>65$ years of age) all patients are at high- or very high-risk even when risk factor levels are not or even marginally elevated.

As age is the most important driver of CVD risk, older patients are almost invariably at higher 10-year risk than younger patients, often regardless of risk factor burden. However, due to diminishing remaining life-expectancy with increasing age, both due to CVD and non-CVD mortality, older persons may have very high 10-year CVD risks, but a relatively limited life expectancy over which a treatment can exert a beneficial cardiovascular risk lowering effect. In short, 10-year CVD risk increases with age while benefit of CVD risk factor treatment in a lifetime perspective diminishes with age. Initiation of preventive therapy based on 10-year CVD-risk may therefore lead to overtreatment in older persons, especially if there is a high risk of competing non-cardiovascular mortality which leads to overestimation of 10-year CVD risk if not accounted for. From the age of 65, the majority of persons is already at high 10-year risk according to SCORE, and as such, there is little use of 10-year risk estimation for a personalized approach to risk management in older persons under the risk-based guidelines. The importance of this is further highlighted by the limited presence of specific advice in the current guidelines on the treatment in older persons, and absence of considerations regarding the relatively limited remaining life expectancy in which therapy can exert its beneficial effects. However, a personalized approach to risk factor treatment in older persons can avoid overtreatment which leads to unnecessary polypharmacy, and a risk of drug-drug interactions and adverse events, such as the risk of orthostatic hypotension and risk of falling in case of blood pressure lowering.

Meanwhile, as the atherosclerotic process begins early in adulthood, important risk factor burden at young age can mean that patients will have very high risk of developing CVD in their lifetime. ${ }^{7}$ coronary artery disease and essential hypertension, are now clearly recognized to begin in childhood. The evidence comes from autopsy studies of cardiovascular-renal changes in the first two decades of life. Cardiovascular risk factors can be identified in children just as in adults and these have a high correlation with the 
anatomic disease. This relationship underscores the importance of risk factor screening of children. Of interest is that clinical risk factors tend to persist within a rank (track However, younger patients at high lifetime risk may have a limited 10 -year risk solely due to their young age even in the presence of high risk factor levels, and are therefore not considered to be eligible for preventive therapy initiation on top of lifestyle optimization. This is counter-intuitive if an aim of cardiovascular risk management is to limit the atherosclerotic process in an early stage.

\section{Predicting lifetime risk and life expectancy free from CVD}

Recent methodological advancements allow estimation of CVD risk in a lifetime perspective. ${ }^{8}$ Methodologically, lifetime estimations use age as the underlying time scale instead of follow-up time from time point 0 (i.e. left truncation), ${ }^{8,9}$ Lifetime estimations can be expressed both as a percentage lifetime CVD risk, or (remaining) event-free life expectancy. Figure 1b shows lifetime CVD risk for patients with different risk profiles as estimated with the The LIFEtime-perspective CardioVascular Disease (LIFE-CVD) model. The LIFE-CVD model is an externally validated competing-risk adjusted Fine and Graymodel for lifetime predictions of major cardiovascular events (MACE; myocardial infarction, stroke, and CVD mortality) and non-CVD mortality for apparently healthy people aged 45 to 90 years. ${ }^{10}$ More details on the model can be found in the Supplementary Methods. In figure $1 b$, it becomes apparent that younger patients with high cardiovascular risk factors have the highest lifetime CVD risks, and this risk diminishes with increasing age.

Event-free life expectancy is an intuitive measure to use in medical decision making and healthcare professional - patient communication and may facilitate the process of shared decision making. For example, a 50-year old patient may have a CVD-free life expectancy up to the age of 80 years, or in other words, a remaining CVD-free life expectancy of 30 years. For a 75-year old patient, a CVD-free life expectancy up to the age of 80 years means a remaining CVD-free life expectancy of only 5 years. This measure thus intuitively helps picture the period of time over which preventive (pharmaco)therapy can exert its effects in an individual patient, which is easy to interpret for clinicians and patients alike.

\section{From prediction algorithms to treatment benefit}

The rationale for risk stratification as currently advocated in international guidelines, is the adagium "the higher the risk, the higher the benefit". In other words, risk is used as a proxy for treatment benefit, which is what is really important when it comes to treatment decisions and communication with patients. 
Therapy benefit for individual patients can be directly estimated by combining prediction algorithms and clinical trial data., ${ }^{911}$ An individual's predicted prognosis (either in terms of 10 -year risk or in a lifetime perspective) can be combined with the relative treatment effect from a trial or meta-analysis to estimate the personalized treatment benefit from therapy. For 10-year risk models, this leads to the calculation of 10 -year absolute risk reductions. ${ }^{11}$ However, risk reductions are presented as percentages, which are abstract numbers that are difficult to interpret for both clinicians and patients. ${ }^{12}$ Furthermore, estimation of 10-year absolute risk reduction has the same limitations in especially younger and older persons as 10-year absolute risks.

Lifetime prediction models can also be combined with trial data to predict individualized treatment effects. Using this method either a lifetime absolute risk reduction (in percentage), or an increase in CVD-free life expectancy (expressed as "years (or months) gained without CVD") is estimated. The latter is an intuitive, easy to understand and easy to communicate measure. As not understanding the effectiveness of therapy is one reason for non-adherence, communication of therapy benefit to a patient and shared decision making may contribute to more effective risk factor treatment. ${ }^{13}$ Individual treatment effect estimations can be performed, free of cost, for individual patients in clinical practice using the currently available lifetime prediction models available on www.U-Prevent.com. ${ }^{10,14,15}$

\section{Lifetime benefit of lipid-lowering, blood pressure-lowering, and smoking cessation}

In figure 2, two-dimensional charts are presented for lifetime benefit from lipid-lowering, blood pressure-lowering and smoking cessation, as estimated with the LIFE-CVD model. The LIFE-CVD model was first used to estimate lifetime risk for CVD and CVD-free life expectancy for individual patients, using the previously described methodology.,10 To estimate the lifetime effects of therapy, the hazard functions of LIFE-CVD were then combined with hazard ratios (HRs) obtained from large meta-analyses for the effects of lipid-lowering, blood pressure-lowering, and smoking cessation (shown in Table 1).9,16

Figure $2 \mathrm{a}$ shows the lifetime treatment effects from $1 \mathrm{mmol} / \mathrm{L}$ LDL-cholesterol lowering for different vascular risk factor profiles in a manner similar to the SCORE risk charts. The lifetime benefit from LDL-cholesterol lowering among the presented spectrum of risk profiles ranges from 0.1 to 2 years increase in CVD-free life expectancy Figure $2 \mathrm{~b}$ shows the lifetime treatment effects from $10 \mathrm{mmHg}$ SBP lowering, ranging from 0 to 2.3 years. Figure $2 \mathrm{c}$ shows the lifetime treatment effect from smoking cessation, ranging from 0.6 to 5.3 years. For all three treatment strategies presented, lifetime benefit is highest in younger patients with a high LDL-cholesterol and/or SBP, and lower in older patients. Lifetime benefit of LDL- and SBP-lowering is higher in non-smokers compared to smokers, as smoking also contributes to an important competing risk of non-CVD mortality. 
Figure 2. Lifetime benefit in terms of life expectancy free from myocardial infarction or stroke gained (A) from $1 \mathrm{mmol} / \mathrm{L}$ LDL-cholesterol lowering; (B) $10 \mathrm{mmHg}$ SBP lowering; and (C) smoking cessation.

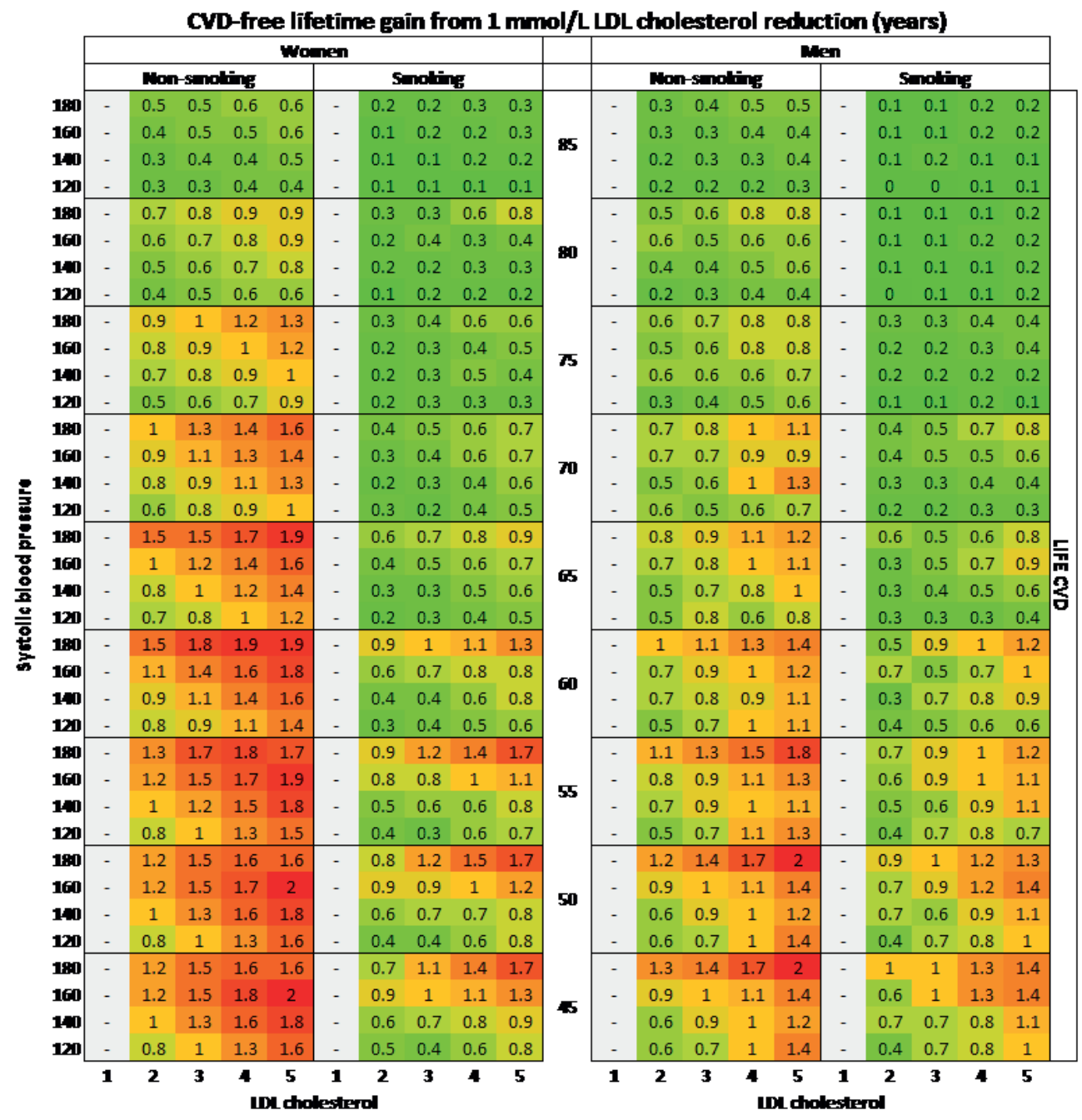

The following population median values were used: BMI of $26 \mathrm{~kg} / \mathrm{m} 2$, no diabetes, no parental history of MI, non-HDL-cholesterol of $0.8 \mathrm{mmol} / \mathrm{L}$ higher than $\mathrm{LDL}$-cholesterol, and HDL-cholesterol of $1.2 \mathrm{mmol} / \mathrm{L}$ for men and $1.4 \mathrm{mmol} / \mathrm{L}$ for women. 
Figure 2. (continued)

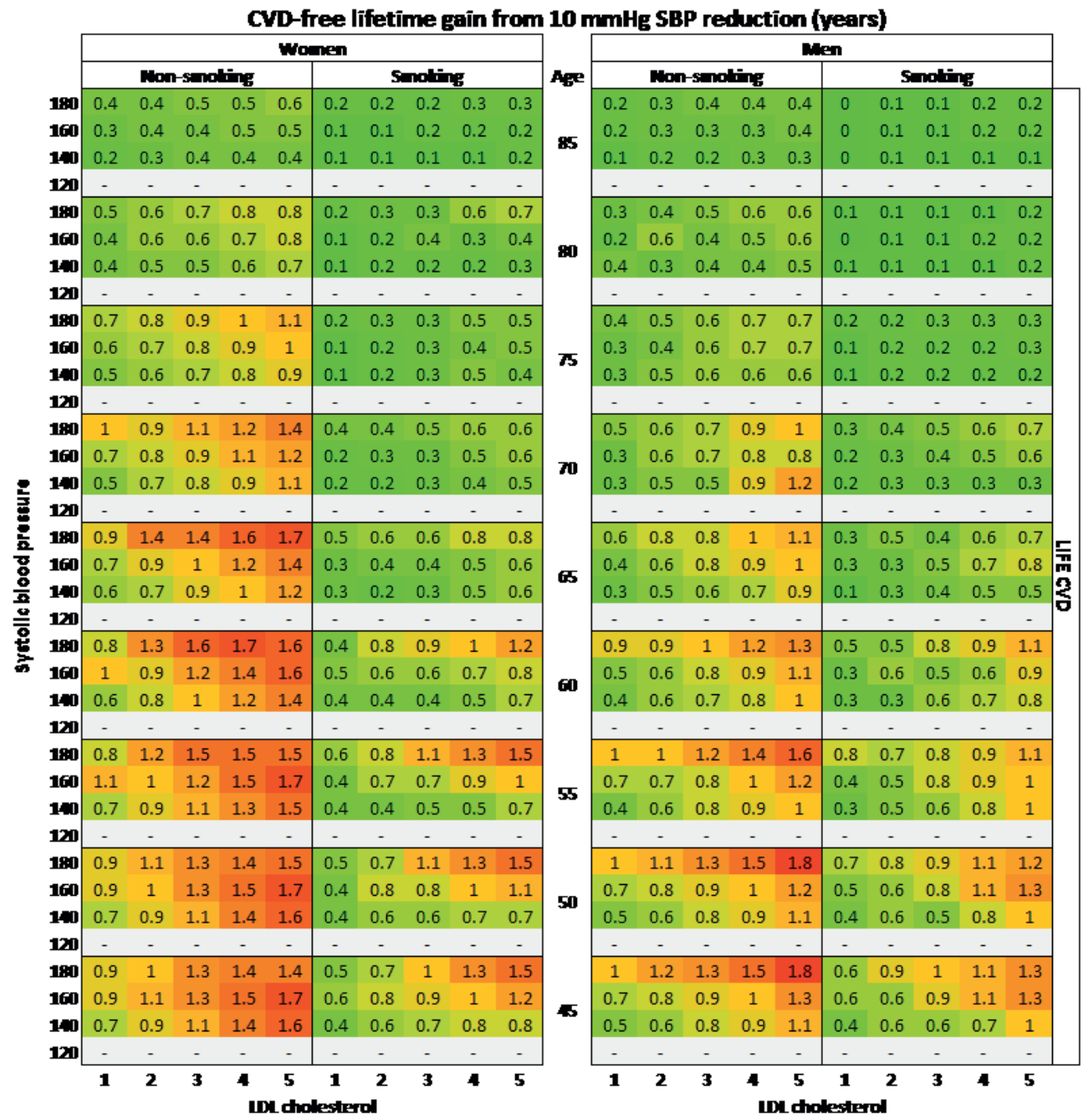

The following population median values were used: BMI of $26 \mathrm{~kg} / \mathrm{m} 2$, no diabetes, no parental history of MI, non-HDL-cholesterol of $0.8 \mathrm{mmol} / \mathrm{L}$ higher than $\mathrm{LDL}$-cholesterol, and HDL-cholesterol of $1.2 \mathrm{mmol} / \mathrm{L}$ for men and $1.4 \mathrm{mmol} / \mathrm{L}$ for women. 
Figure 2. (continued)

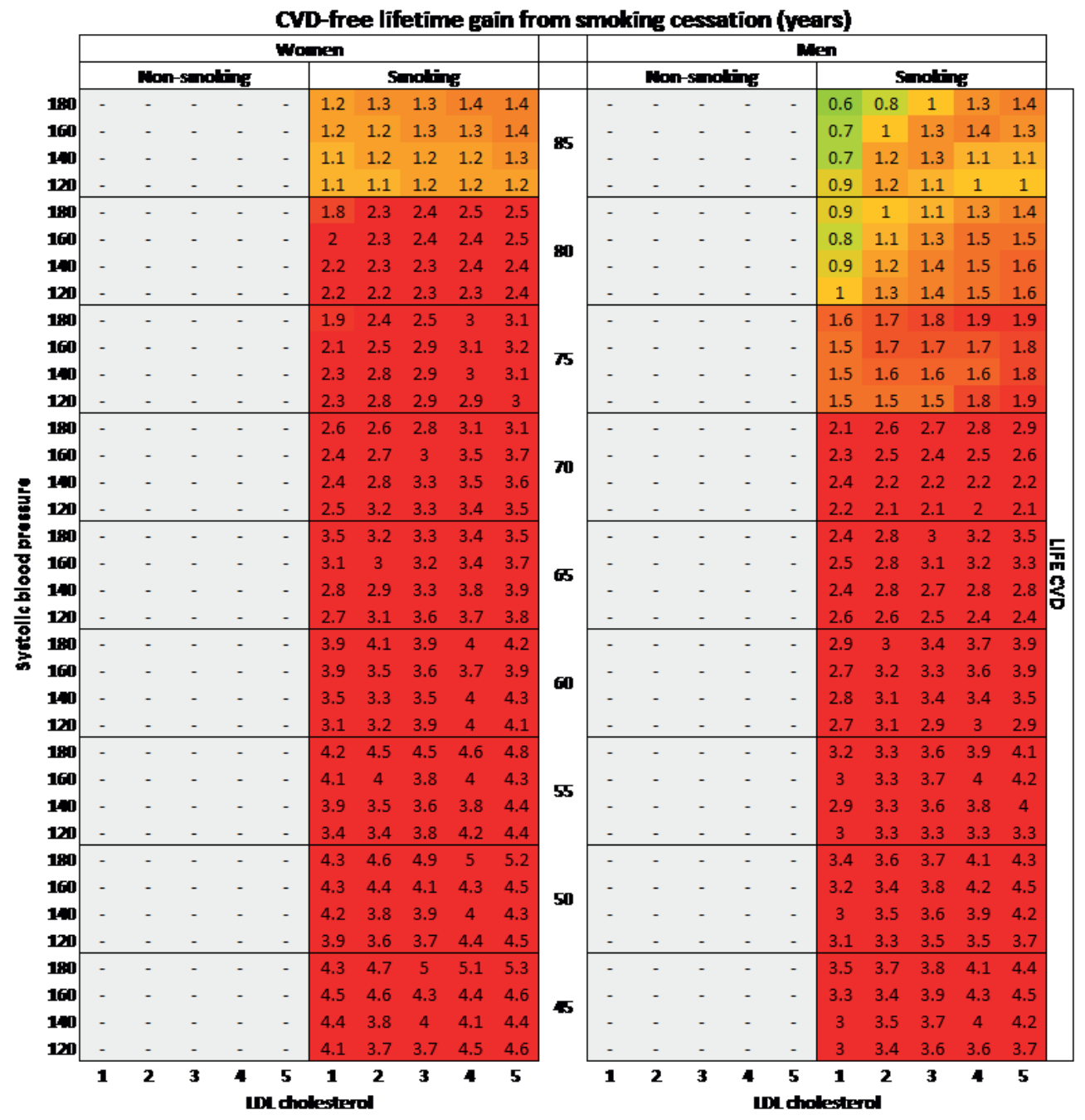

The following population median values were used: BMI of $26 \mathrm{~kg} / \mathrm{m} 2$, no diabetes, no parental history of MI, non-HDL-cholesterol of $0.8 \mathrm{mmol} / \mathrm{L}$ higher than LDL-cholesterol, and HDL-cholesterol of $1.2 \mathrm{mmol} / \mathrm{L}$ for men and $1.4 \mathrm{mmol} / \mathrm{L}$ for women. 
Figure 3 shows an example of how 10-year risk, lifetime risk, and remaining life expectancy, and the 10-year absolute risk reduction (ARR) and lifetime benefit (in this example from blood pressure-lowering) are influenced by age in patients with the exact same vascular risk profile. While the 10 -year risk is much higher in the 85 year-old compared to the 50 year-old in this example (15.9\% compared to 1.6\%), the lifetime benefit from lowering his blood pressure from $160 \mathrm{mmHg}$ to $140 \mathrm{mmHg}$ gives a much lower lifetime benefit (5 months gained without CVD for the 85-year old compared to 20 gained without CVD months for the 50-year old). This is exemplary of how short-term risk and lifetime benefit change with age.

Thus, the figures illustrate that using a lifetime approach younger persons with a high LDL cholesterol and/or SBP benefit most from cholesterol-lowering or blood pressure-lowering treatment. Implementing a lifetime benefit approach in daily practice, as compared to the risk-based approach, would generally shift the focus for cardiovascular risk management from older people to younger people with a high burden of cardiovascular risk factors.

Figure 3. Influence of age on (A) 10-year risk of fatal CVD, lifetime risk of CVD, and remaining CVD-free life expectancy; and (B) 10-year absolute risk reduction (ARR) for fatal CVD, and lifetime benefit in terms of months without CVD gained from $20 \mathrm{mmHg}$ systolic blood pressure $(\mathrm{SBP})$ reduction, assuming otherwise identical risk profiles.
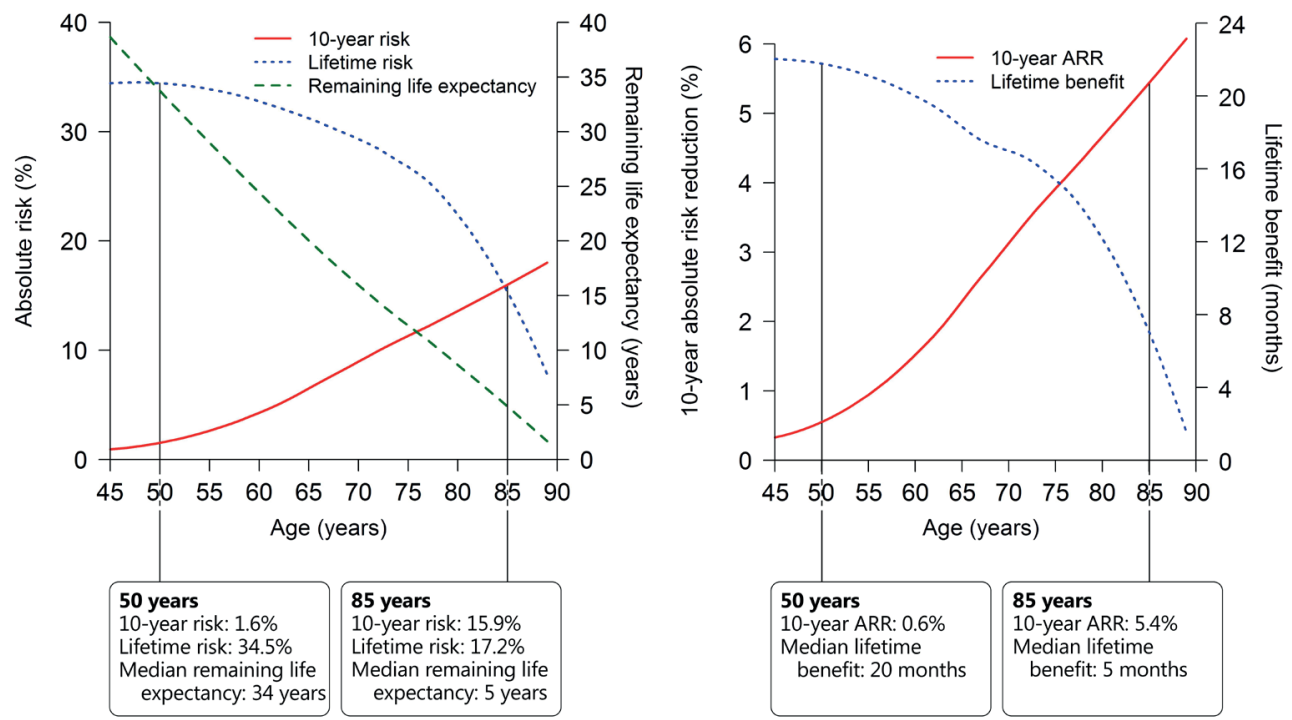

Risk profile: Male, non-smoker, SBP $160 \mathrm{mmHg}, B M I 26 \mathrm{~kg} / \mathrm{m} 2, \mathrm{LDL}$ cholesterol $3 \mathrm{mmol} / \mathrm{L}$, HDL cholesterol 1.2 $\mathrm{mmol} / \mathrm{L}$, eGFR $70 \mathrm{ml} / \mathrm{min}$, no diabetes, no family history of premature CVD. 


\section{Considerations in clinical practice}

While 10-year CV risk estimation with currently available risk scores (SCORE, ASCVD) may be used for risk stratification in middle aged persons, the use of 10-year risk estimation is generally not practical for treatment decisions in younger ( $<50$ years) and older ( $>65$ years) persons. Lifetime treatment effect estimations can then be used to make personalized preventive treatment decisions instead. As illustrated in Figure 3, the median lifetime benefit relates to the remaining life expectancy. As expected, the longer the remaining life expectancy, the longer the median lifetime benefit from preventive medication. While this means that there is more healthy life to be gained, there is also a much longer period over which preventive medication has to be taken. Therefore, in clinical practice, after answering the question, "What is the lifetime benefit from this treatment?", treatment decisions are based on the patient-clinician discussion of the question, "Is this lifetime benefit enough?". In other words, what is the 'return on investment' from lifestyle intervention and preventive pharmacotherapy? Preventive pharmacotherapy such as lipid-lowering and blood pressure-lowering should likely be used for a very long time, potentially even lifelong. If the lifetime benefit is 1 year, the return on investment is likely to be judged very differently for a 45 year old patient with a current life expectancy of 80 years, compared to a 75 year old with the same life expectancy. Secondly, there is large variation in what patients - and clinicians - consider a worthwhile lifetime benefit, ${ }^{17}$ especially with statins, which have an (albeit falsely) high perceived risk of adverse events. This is why patientclinician communication and shared decision making are very important in the context of cardiovascular risk management. Box 1 gives a summary of considerations for clinical practice when using lifetime treatment effect predictions.

\section{Box 1. Considerations when using lifetime treatment effect prediction in clinical practice}

- How much months (or years) free from CVD can be gained with a given treatment option, or combination of treatment options?

- Preventive medication often has to be taken lifelong. What is the remaining life expectancy in daily practice?

- What 'return on investment' does the patient consider worthwhile (i.e. what lifetime benefit does the patient consider enough for the number of years the treatment should likely be taken)?

- How does the individual patient perceive the burden of this preventive treatment?

- What are the adverse events that can be expected, and will the expected benefit outweigh these risks?

- How much does the given treatment option cost? (especially for individual patients when certain treatments are not reimbursed by insurance) 


\section{Additional remarks}

For lifetime estimations, the assumption is made that important risk factor burden at young age means that patients will have very high risk of developing CVD in their lifetime. This notion is supported by Mendelian randomization studies, which have shown that lifelong genetic exposure to even small differences in SBP and LDL-cholesterol is associated with marked differences in cardiovascular risk, even with a magnitude larger than expected from the combined treatment effects from pharmacological risk factor reduction. ${ }^{18}$ As the currently available lifetime models are not based on data with life-long follow-up, it is possible that in younger persons, the lifetime risk and lifetime treatment effects in patients with high risk factor levels are underestimations of their actual risk. This would mean that the real lifetime treatment effects in younger patients may be even larger than currently reported.

Some potential limitations of the methods used in this paper should be considered. First, two different risk scores were used for patients aged 65 or younger (SCORE) versus patients aged over 65 years (older person-specific risk score), because there is no risk score used routinely in daily clinical practice that covers the entire age range. Furthermore, SCORE is not adjusted for competing non-CVD mortality risk. This means that, especially in the older SCORE age range(>60 years) where non-CVD mortality becomes more important, there might be some overestimation of the CVD risk. ${ }^{19-21}$ This is reflected in the fact that in the risk charts, some of the 70 year old patients have lower risks than the corresponding 65 year olds. A third limitation is that the two-dimensional score chart is a simplified version of the models which does not use all available prognostic information. Using the full model for individual patients yields more precise estimates of CV risk than the simplified risk charts with a wide range of potential risk. Furthermore, the treatment benefit charts only show the benefit of $1 \mathrm{mmol} / \mathrm{L}$ LDL reduction and $10 \mathrm{mmHg}$ SBP reduction, while different treatment goals may be considered in clinical practice. The two-dimensional risk and benefit charts presented in this paper are thus primarily for illustration.

\section{Recommendations and conclusions}

A potential approach to using CVD prediction in persons without established CVD in the future could be to combine short-term (e.g. 10-year) risk with lifetime risk, especially in middle-aged persons. When the SCORE risk is high below the age of 65 years, this is enough for an indication for pharmacotherapy. When the SCORE risk is low, however, and especially in younger adults, lifetime risk and lifetime treatment effects can be used to establish who would benefit from preventive pharmacotherapy on a lifetime perspective. In older adults (>65 years), 10-year risk estimation with an older person-specific risk 
score could be used to illustrate the high CVD risk in patient-clinician communication, while lifetime benefit could be used to facilitate treatment decisions in a shared decision process, thereby avoiding overtreatment when treatment decisions are based on 10-year risk thresholds. Alternatively, a lifetime risk and lifetime benefit approach could be the first step instead of 10-year risk estimations. A disadvantage could be, that healthcare professionals and patients are used to working with 10-year risks, and, secondly, that no formal thresholds yet exist for treatment decisions based on lifetime treatment effect estimations. A counter argument for that could be that it is really up to each individual patient and the healthcare professional to consider if an expected lifetime benefit of treatment is worthwhile for starting such a therapy. In essence there are no 'wrong' decisions.

In conclusion, calculating individual lifetime benefit expressed as extra CVD-free life from treatment provides an easy to understand and easy to communicate estimate that can be used in patient - healthcare provider discussion. Treatment decisions for risk factor modification based on estimated lifetime benefit from preventive therapy rather than estimated absolute 10-year risk may lead to the treatment of younger high-risk patients with higher risk factor levels, rather than older patients, in the primary prevention of cardiovascular disease. Use of lifetime benefit predictions in clinical practice will likely support the process of shared decision-making. 


\section{References}

1. Piepoli MF, Hoes AW, Agewall S, Albus C, Brotons C, Catapano AL, et al. 2016 European guidelines on cardiovascular disease prevention in clinical practice: the Sixth Joint Task Force of the European Society of Cardiology and Other Societies on Cardiovascular Disease Prevention in Clinical Practice. Eur Heart J. 2016;37(29):2315-81.

2. Goff DCJ, Lloyd-Jones DM, Bennett G, Coady S, D'Agostino RB, Gibbons R, et al. 2013 ACC/ AHA guideline on the assessment of cardiovascular risk: a report of the American College of Cardiology/American Heart Association Task Force on Practice Guidelines. Circulation. 2014;129(25):S49-73.

3. Conroy RM, Group on behalf of the S project, Pyörälä K, Group on behalf of the S project, Fitzgerald AP, Group on behalf of the S project, et al. Estimation of ten-year risk of fatal cardiovascular disease in Europe: the SCORE project. Eur Heart J. 2003;24(11):987-1003.

4. D'Agostino RB, Vasan RS, Pencina MJ, Wolf PA, Cobain M, Massaro JM, et al. General cardiovascular risk profile for use in primary care: The Framingham heart study. Circulation. 2008;117(6):743-53.

5. Piepoli MF, Hoes AW, Agewall S, Albus C, Brotons C, Catapano AL, et al. 2016 European Guidelines on cardiovascular disease prevention in clinical practice. Eur Heart J. 2016;37(29):2315-81.

6. Stam-Slob MC, Visseren FLJ, Jukema J, van der Graaf Y, Poulter NR, Gupta A, et al. Personalized absolute benefit of statin treatment for primary or secondary prevention of vascular disease in individual elderly patients. Clin Res Cardiol. 2017;106(1):58-68.

7. Berenson GS, Srinivasan SR, Hunter SM, Nicklas TA, Freedman DS, Shear CL, et al. Risk factors in early life as predictors of adult heart disease: the Bogalusa Heart Study. Am J Med Sci. 1989;298(3):141-51.

8. Joint British Societies' consensus recommendations for the prevention of cardiovascular disease (JBS3). Heart. 2014;100(Suppl 2):ii1 LP-ii67.

9. Dorresteijn JAN, Kaasenbrood L, Cook NR, van Kruijsdijk RCM, van der Graaf Y, Visseren FLJ, et al. How to translate clinical trial results into gain in healthy life expectancy for individual patients. BMJ. 2016;352:i1548.

10. Jaspers NEM, Blaha MJ, Matsushita K, van der Schouw YT, Wareham NJ, Khaw K-T, et al. Prediction of individualized lifetime benefit from cholesterol lowering, blood pressure lowering, antithrombotic therapy, and smoking cessation in apparently healthy people. Eur Heart J. 2019;31:1-10.

11. Dorresteijn JAN, Visseren FLJ, Ridker PM, Wassink AMJ, Paynter NP, Steyerberg EW, et al. Estimating treatment effects for individual patients based on the results of randomised clinical trials. BMJ. 2011 Oct 3;343(1):d5888-d5888.

12. Gigerenzer G. Simple tools for understanding risks: from innumeracy to insight. BMJ. 2003;327(7417):741-4.

13. Wouters H, Van Dijk L, Geers HCJ, Winters NA, Van Geffen ECG, Stiggelbout AM, et al. 
Understanding Statin Non-Adherence: Knowing Which Perceptions and Experiences Matter to Different Patients. Catapano A, editor. PLoS One. 2016;11(1):e0146272.

14. Berkelmans GFN, Gudbjörnsdottir S, Visseren FLJ, Wild SH, Franzen S, Chalmers J, et al. Prediction of individual life-years gained without cardiovascular events from lipid, blood pressure, glucose, and aspirin treatment based on data of more than 500000 patients with Type 2 diabetes mellitus. Eur Heart J. 2019;1-10.

15. Kaasenbrood L, Bhatt DL, Dorresteijn JA, Wilson PW, D'Agostino RB, Massaron JM, et al. Estimated life-expectancy without recurrent cardiovascular events in patients with vascular disease: the REACH-SMART model. J Am Hear. 2018;7:e009217.

16. Lloyd-Jones DM, Huffman MD, Karmali KN, Sanghavi DM, Wright JS, Pelser C, et al. Estimating Longitudinal Risks and Benefits From Cardiovascular Preventive Therapies Among Medicare Patients. J Am Coll Cardiol. 2017;69(12):1617-36.

17. Jaspers NEM, Visseren FLJ, Numans ME, Smulders YM, Van Loenen Martinet FA, Van Der Graaf $Y$, et al. Variation in minimum desired cardiovascular disease-free longevity benefit from statin and antihypertensive medications: A cross-sectional study of patient and primary care physician perspectives. BMJ Open. 2018;8(5):1-9.

18. Ference BA, Bhatt DL, Catapano AL, Packard CJ, Graham I, Kaptoge S, et al. Association of Genetic Variants Related to Combined Exposure to Lower Low-Density Lipoproteins and Lower Systolic Blood Pressure with Lifetime Risk of Cardiovascular Disease. JAMA - J Am Med Assoc. 2019;322(14):1381-91.

19. Wolbers M, Koller MT, Witteman JCM, Steyerberg EW. Prognostic models with competing risks: methods and application to coronary risk prediction. Epidemiology. 2009;20(4):555-61.

20. Berry SD, Ngo L, Samelson EJ, Kiel DP. Competing risk of death: an important consideration in studies of older adults. J Am Geriatr Soc. 2010;58(4):783-7.

21. Austin PC, Lee DS, Fine JP. Statistical Primer for Cardiovascular Research Introduction to the Analysis of Survival Data in the Presence of Competing Risks. 2016;601-9.

22. Ettehad D, Emdin CA, Kiran A, Anderson SG, Callender T, Emberson J, et al. Blood pressure lowering for prevention of cardiovascular disease and death: a systematic review and metaanalysis. Lancet. 2016;387(10022):957-67.

23. Baigent C, Blackwell L, Emberson J, Holland LE, Reith C, Bhala N, et al. Efficacy and safety of more intensive lowering of LDL cholesterol: A meta-analysis of data from 170000 participants in 26 randomised trials. Lancet. 2010;376(9753):1670-81.

24. Mons U, Muezzinler A, Gellert C, Schottker B, Abnet CC, Bobak M, et al. Impact of smoking and smoking cessation on cardiovascular events and mortality among older adults: meta-analysis of individual participant data from prospective cohort studies of the CHANCES consortium. BMJ. 2015;350(apr20 2):h1551-h1551.

25. Gellert C, Schöttker B, Brenner H. Smoking and All-Cause Mortality in Older People. Arch Intern Med. 2012 Jun 11;172(11):837-44. 


\section{Supplementary methods}

\section{Estimation of $\mathbf{1 0}$-year absolute risk}

10 -year absolute risk was calculated using the Systematic COronary Risk Evaluation (SCORE) risk score and an older person-specific risk score. ${ }^{1,2}$ SCORE is a Weibull model derived in more than 200,000 patients in a pooled dataset with cohort studies from 12 European countries. The risk chart uses the following predictors: sex, current smoking, SBP, and total cholesterol/HDL cholesterol ratio, with age used to define the hazard function. ${ }^{3}$ It estimates the 10-year risk of fatal CVD for patients aged 40 to 69 years. For the current study, the risk prediction model for low-risk countries was used. A Fine and Gray competing risk (i.e. non-vascular death) adjusted, older person-specific risk model was used for the prediction of 10-year risk of major cardiovascular events (MACE; a composite endpoint of stroke, myocardial infarction, and cardiovascular mortality) in older persons. ${ }^{2}$ It was developed in the PROspective Study of Pravastatin in Elderly at Risk trial population in patients aged $>70$ years, ${ }^{3}$ and externally validated in the Anglo-Scandinavian Cardiac Outcomes Trial - Lipid-Lowering Arm trial for patients without vascular disease. ${ }^{4}$ The model is based on the following baseline predictors: age, sex, current smoking, diabetes mellitus, total number of medications used, SBP, low-density lipoprotein (LDL)-cholesterol, high-density lipoprotein (HDL)-cholesterol, estimated glomerular filtration rate (eGFR), and the presence of polyvascular disease in case of history of CVD. ${ }^{2}$ These risk models can be combined with the hazard ratios described in Table 1 to calculate absolute risk reductions from these therapies. Absolute risk reduction (ARR) is defined as the individual absolute off-treatment risk minus individual absolute on-treatment risk.

\section{Lifetime risk and lifetime treatment effect estimations}

Lifetime treatment effects were estimated using the LIFEtime-perspective CardioVascular Disease (LIFECVD) model, which was derived in 6,715 participants from the Multi-Ethnic Study of Atherosclerosis (MESA) and validated in 62,808 participants from the Atherosclerosis Risk in Communities (ARIC), Heinz Nixdorf Recall (HNR), and the European Prospective Investigation into Cancer and Nutrition (EPIC) Netherlands and Norfolk studies. ${ }^{5}$ The model is based on the following baseline characteristics: sex, systolic blood pressure (SBP), non-high density lipoprotein (HDL)-cholesterol, body mass index (BMI), smoking status (current, former, or never smoker), diabetes mellitus, and parental history of premature myocardial infarction (prior to age 60).

The LIFE-CVD model can used to estimate lifetime risk for CVD and CVD-free life expectancy for individual patients, using previously described methodology. ${ }^{6}$ Lifetime risk is defined as the risk of having a CVD-event before the age of 90. CVD-free life expectancy is defined as the age at which the cumulative survival probability becomes lower than 50\%. Remaining CVD-free life expectancy is defined as the CVD-free life expectancy minus current age of the individual.

The gain in CVD-free life expectancy for each of these three treatments was estimated as the difference between on- and off-treatment median CVD-free life expectancy. In patients where the life expectancy with treatment, without treatment, or both, exceeds 90 years, this approach cannot be 
used as the survival curve does not drop below 50\%. In that case the percentage on the off-treatment survival curve at age 90 was compared with the age where the same percentage was found on the on-treatment survival curve to calculate the treatment benefit. For example, if the survival curve at 90 years is at $60 \%$, the treatment benefit is the difference between 90 and the age at which the ontreatment survival curve is at $60 \%$ was used.

\section{References used in supplementary methods}

1. Conroy RM, Group on behalf of the S project, Pyörälä K, Group on behalf of the S project, Fitzgerald AP, Group on behalf of the $S$ project, et al. Estimation of ten-year risk of fatal cardiovascular disease in Europe: the SCORE project. Eur Heart J. 2003;24(11):987-1003.

2. Stam-Slob MC, Visseren FLJ, Jukema J, van der Graaf Y, Poulter NR, Gupta A, et al. Personalized absolute benefit of statin treatment for primary or secondary prevention of vascular disease in individual elderly patients. Clin Res Cardiol. 2017;106(1):58-68.

3. Shepherd J, Blauw GJ, Murphy MB, Bollen ELEM, Buckley BM, Cobbe SM, et al. Pravastatin in elderly individuals at risk of vascular disease (PROSPER): a randomised controlled trial. Lancet. 2002;360(9346):1623-30.

4. Dahlof B, Sever PS, Poulter NR, Wedel H, Beevers DG, Caulfield M, et al. Prevention of cardiovascular events with an antihypertensive regimen of amlodipine adding perindopril as required versus atenolol adding bendroflumethiazide as required, in the Anglo-Scandinavian Cardiac Outcomes Trial-Blood Pressure Lowering Arm (ASCOT-B. Lancet. 2005;366(9489):895-906.

5. Jaspers NEM, Blaha MJ, Matsushita K, van der Schouw YT, Wareham NJ, Khaw K-T, et al. Prediction of individualized lifetime benefit from cholesterol lowering, blood pressure lowering, antithrombotic therapy, and smoking cessation in apparently healthy people. Eur Heart J. 2019;31:1-10.

6. Dorresteijn JAN, Kaasenbrood L, Cook NR, van Kruijsdijk RCM, van der Graaf Y, Visseren FLJ, et al. How to translate clinical trial results into gain in healthy life expectancy for individual patients. BMJ. 2016;352:i1548. 


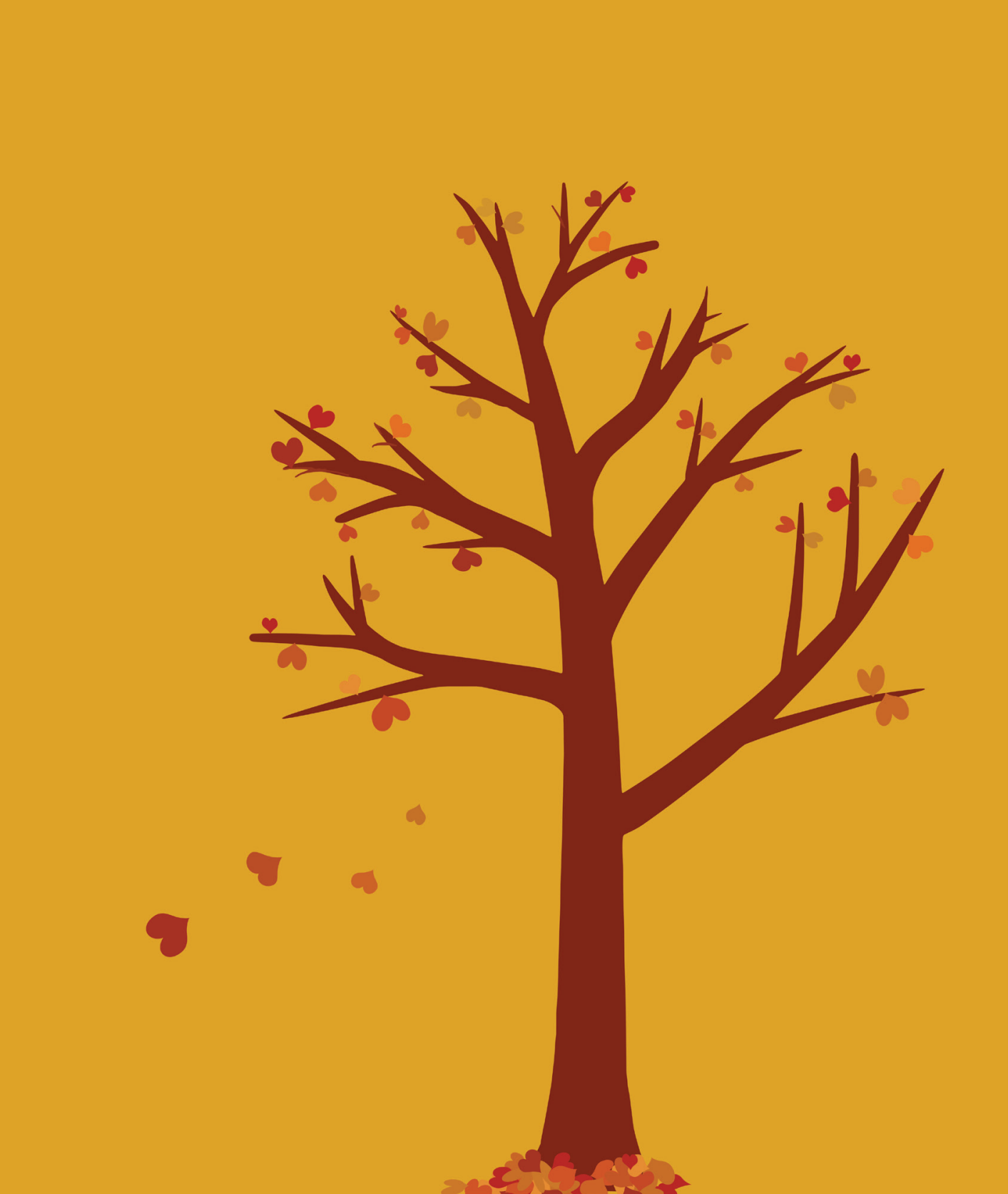




\title{
Estimating individual lifetime benefit and bleeding risk of adding rivaroxaban to aspirin for patients with stable cardiovascular disease: results from the COMPASS trial
}

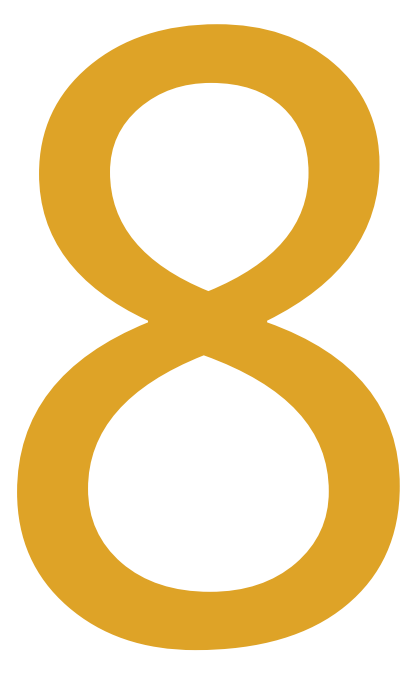

\author{
Tamar I. de Vries \\ John W. Eikelboom \\ Jackie Bosch \\ Jan Westerink \\ Jannick A.N. Dorresteijn \\ Marco Alings

Eur Heart J. 2019;40(46):3771-3778a. 


\section{ABSTRACT}

\section{Background}

Adding rivaroxaban to aspirin in patients with stable atherosclerotic disease reduces the recurrence of cardiovascular disease (CVD) but increases the risk of major bleeding. The aim of this study was to estimate the individual lifetime treatment benefit and harm of adding low-dose rivaroxaban to aspirin in patients with stable cardiovascular disease.

\section{Methods and results}

Patients with established CVD from the COMPASS trial $(n=27390)$ and SMART prospective cohort study ( $n=8139$ ) were used. Using the pre-existing lifetime SMART-REACH model for recurrent $C V D$, and a newly developed Fine and Gray competing risk-adjusted lifetime model for major bleeding, individual treatment effects from adding low-dose rivaroxaban to aspirin in patients with stable CVD were estimated, expressed in terms of (i) life-years free of stroke or myocardial infarction (MI) gained; and (ii) life-years free from major bleeding lost. Calibration of the SMART-REACH model for prediction of recurrent CVD events in the COMPASS study was good. The major bleeding risk model as derived in the COMPASS trial showed good external calibration in the SMART cohort. Predicted individual gain in life expectancy free of stroke or MI from added low-dose rivaroxaban had a median of 16 months (range 1-48 months), while predicted individualized lifetime lost in terms of major bleeding had a median of 2 months (range $0-20$ months).

\section{Conclusions}

There is a wide distribution in lifetime gain and harm from adding low-dose rivaroxaban to aspirin in individual patients with stable CVD. Using these lifetime models, benefits and bleeding risk can be weighed for each individual patient, which could facilitate treatment decisions in clinical practice. 


\section{Introduction}

Patients with a history of cardiovascular disease (CVD) remain at elevated risk for recurrent vascular events despite preventive strategies, including lifestyle changes, lipid-lowering, blood pressure-lowering and the use of antiplatelet therapy. ${ }^{1-3}$ The "Cardiovascular Outcomes for People Using Anticoagulation Strategies" (COMPASS) trial showed that adding rivaroxaban $2.5 \mathrm{mg}$ twice-daily to aspirin was superior to aspirin alone in prevention of major cardiovascular events (MACE) and all-cause mortality in a secondary prevention setting. Lowering of CVD risk by adding rivaroxaban to aspirin was achieved accompanied by a slight increase in bleeding risk. ${ }^{4}$

In clinical practice, clinicians face the challenge of translating average efficacy and safety results from clinical trials like COMPASS to individual patients. ${ }^{5}$ Trial results are usually reported in terms of average relative risk reductions for the primary outcome, and relative risk increases of adverse events. However, due to different patient characteristics, the absolute individual treatment effect differs between individuals. ${ }^{5}$ Likewise, the absolute risk of adverse events, e.g. major bleeding with anticoagulants, differs between individual patients. This means that one patient may have a large treatment benefit with a low risk of bleeding, while another might have little treatment benefit with a high risk of major bleeding. Predicting the individual lifetime treatment benefit and harm in COMPASS has the potential to identify those patients who will benefit most from adding rivaroxaban to aspirin, while having an acceptable risk of major bleeding.

The objective of the present study was to estimate the absolute individual lifetime treatment benefit and harm of low-dose rivaroxaban added to aspirin for individual patients with stable CVD in terms of: (1) life-years without myocardial infarction or stroke gained; and (2) life-years free from major bleeding lost.

\section{Methods}

\section{Study populations}

The COMPASS trial (registration number: NCT01776424) was a double-blind, randomized, placebo-controlled clinical trial comparing aspirin alone with rivaroxaban $2.5 \mathrm{mg}$ twicedaily with aspirin or rivaroxaban $5 \mathrm{mg}$ twice-daily without aspirin for the prevention of MACE in 27,395 participants from 33 countries. In the current study, data from 27,390 patients with a history of stable atherosclerotic vascular disease were used. The Second Manifestations of ARTerial disease (SMART) study is an ongoing prospective cohort study of patients with established CVD or cardiovascular risk factors at the University Medical Center Utrecht. For the current study, data were used from 8,139 patients with clinically manifest CVD enrolled between 1996 and 2017. Detailed descriptions of both studies have 
been published elsewhere.6,7 Both studies complied with the Declaration of Helsinki, were approved by institutional review boards and all participants provided written informed consent. All included study participants were people aged $>18$ years with clinically manifest vascular disease, defined as either coronary artery disease (CAD) or peripheral artery disease, and in the SMART cohort also as cerebrovascular disease or abdominal aortic aneurysm. A comprehensive overview of eligibility criteria for the original studies is provided in Supplemental Table 1a.

\section{Outcomes}

CVD was defined as myocardial infarction, stroke, or vascular mortality. Non-vascular mortality was defined as death without a cardiovascular cause. Major bleeding was defined according to the International Society on Thrombosis and Haemostasis (ISTH) criteria: fatal bleeding, symptomatic bleeding in a critical area or organ, bleeding causing a fall in hemoglobin level of $\geq 20 \mathrm{~g} / \mathrm{L}(>1.24 \mathrm{mmol} / \mathrm{L})$, and/or leading to transfusion of $\geq 2$ units of whole blood or red cells. ${ }^{8}$ Endpoint definitions are described in Supplemental Table 1b. Outcome assessment in COMPASS was blinded to randomization.

\section{External validation of SMART-REACH model}

The SMART-REACH prediction algorithm is a previously derived, externally validated competing-risk adjusted Fine and Gray-model for lifetime predictions for MACE and noncardiovascular death in patients with clinically manifest vascular disease (Supplemental Methods). ${ }^{9}$ After adjusting for differences in underlying event rates, external validation of this model in COMPASS was performed, using the c-statistic for discrimination and plots of predicted-versus-observed 2-year risk for calibration (detailed descriptions of the used methodology provided in the Supplemental Methods).

\section{Development of a prediction model for major bleeding}

In the COMPASS trial, we developed two complementary Fine and Gray competing riskadjusted subdistribution-hazard functions for cause-specific estimates of the cumulative

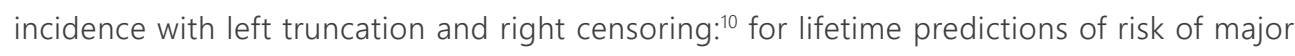
bleeding, and for competing mortality. These statistical methods have been previously described in detail.9.11-14 In short, age was used as underlying time-function. Patients contribute data to the survival function from age of study entry to age of study exit (either time of event or censoring). This results in overlapping observations, allowing for lifetime predictions to be made across the range of baseline ages. Estimates derived from these models are limited by age distribution of study participants rather than follow-up time. As predictions can be unstable when the number of people and events of interest are limited in a specific age group, the age-range was limited to 45 to 90 years. Predictors were prespecified, based on previous bleeding models, ${ }^{15-17}$ and selected on availability in the studies 
and in clinical practice: age, gender, ethnicity, geographical region, current smoking, systolic blood pressure (SBP), number of CVD locations, diabetes mellitus, history of congestive heart failure, history of bleeding requiring transfusion, serum creatinine, and total cholesterol. Furthermore, a dummy variable for treatment with rivaroxaban-plus-aspirin or rivaroxaban alone was added to the model to adjust for treatment-related increases in bleeding risk. Continuous predictors were truncated at the $1^{\text {st }}$ and $99^{\text {th }}$ percentile to limit the effect of outliers. Whether the association of continuous predictors with the outcome variable is loglinear was assessed with restricted cubic splines; to improve the robustness of the model, transformation was applied when this improved model fit, based on Akaike's Information Criterion. ${ }^{18}$ The proportional hazards assumptions were checked by visually assessing the correlations between scaled Schoenfeld residuals for the various predictors and age.

\section{Lifetime predictions of event-free survival for individual patients}

Life expectancy without recurrent cardiovascular events and without major bleeding was estimated using the SMART-REACH model and major bleeding risk model, respectively. Beginning at age at baseline for each individual participant, the risk of the event of interest $\left(a_{t}\right)$ and the risk of the competing event $\left(b_{t}\right)$ was estimated for each future life-year. Next, the probability of being healthy and alive at interval $t\left(\mathrm{e}_{\mathrm{t}+1}\right)$ was calculated by multiplying the survival probability at the beginning of each life-year $\left(e_{t}\right)$ by the event-free survival probability during that year $\left(1-a_{t}-b_{t}\right)$. This process is repeated until the maximum age of 90 years. These predictions together form an individual life table with one-year intervals. Event-free life-expectancy was defined as median estimated survival, the age where the predicted individual survival curve is 50\%. 10-year risk (or other durations of interest) of the event of interest can be predicted by calculating the cumulative cause-specific eventrisk truncated at 10 years after age at baseline.

\section{Model validation}

Internal validity of the major bleeding risk model was assessed with calibration plots of predicted-versus-observed 2-year risk in the COMPASS trial; c-statistics were obtained using bootstrapping with 1000 bootstrap samples. External validity of the major bleeding risk model was tested in the SMART cohort at 10-year follow-up in patients without oral anticoagulants. Calibration plots were used to assess goodness-of-fit for bleeding-free survival, bleeding events, and non-bleeding mortality functions after recalibration based on the incidence rate of bleeding and non-bleeding mortality using the expected-versusobserved ratio; discrimination was assessed using c-statistics. 


\section{Individual treatment effect predictions}

The competing risk adjusted Cox proportional hazard function for the prediction of the event of interest from the SMART-REACH and major bleeding risk model were combined with hazard ratios (HR) from the COMPASS trial for the treatment arm aspirin-plusrivaroxaban according to previously described methods. ${ }^{9,12,19,20}$ These HRs were applied to the 1-year estimates of respectively the SMART-REACH model and the major bleeding model by adding the logarithm of the HR to the linear predictor of the model. Because these methods make use of life tables, any gain or loss in event-free survival will be adjusted for the competing risks because the time at risk for the competing event changes. For the CVD survival function, the HR for added rivaroxaban is $0.76 .{ }^{4}$ For the major bleeding function, to account for the decrease in increased risk from the addition of rivaroxaban to aspirin after the first year, several HRs were used: 2.32 in the first year after commencement of the added rivaroxaban, 1.19 in the second year, and 1.05 after more than two years. ${ }^{21}$ Heterogeneity of treatment effect across baseline risk for disease was assessed by fitting a model including an interaction term between the linear predictor and treatment allocation for all models. ${ }^{5}$

The median CVD-free life expectancy with aspirin was estimated for each patient. Treatment benefit or harm for individual patients, the expected lifetime benefit or harm when adding rivaroxaban to aspirin when compared to aspirin only, was defined as the patient's predicted event-free life expectancy when using aspirin (baseline risk with standard of care) minus the patient's predicted event-free life expectancy when adding low-dose rivaroxaban to aspirin. Similarly, the 10-year absolute event-risk reduction and increase for individual persons were estimated by calculating the difference between the predicted 10-year event-risk with and without added rivaroxaban.

Missing data ( $<1 \%$ in both COMPASS and SMART) were imputed by single imputation using predictive mean matching (areglmpute-algorithm in R, Hmisc-package). All analyses were conducted with R statistical software V.3.4.1 (www.r-project.org).

To enable the use of the SMART-REACH lifetime model and the major bleeding risk model in clinical practice, we have developed a calculator that allows for the estimation of the potential gain in life expectancy free from cardiovascular disease, or loss in life expectancy free from major bleeding due to adding rivaroxaban to the treatment strategy for individual patients, as well as the 10-year absolute changes in risk (Supplemental material online, or with the online calculator on www.U-Prevent.com). 


\section{Results}

Baseline characteristics of the study populations are shown in Table 1. SMART participants were more often current smokers (47\% versus $21 \%$ ), while in COMPASS more patients had diabetes mellitus (38\% versus 17\%). In SMART, more patients were included with cerebrovascular disease (30\% versus $4 \%$ ), and in COMPASS, more patients were included with CAD (91\% versus 61\%). In the COMPASS trial, a total of 1,323 cardiovascular events, 499 non-cardiovascular deaths, and 497 major bleedings were observed during a median follow-up of 1.9 years (interquartile range [IQR] 1.3-2.5). In the SMART cohort, 1,568 cardiovascular events, 907 non-cardiovascular deaths, and 335 major bleedings occurred during a median follow-up of 7.6 years (IQR 3.9-11.7).

\section{Validation of the SMART-REACH model in COMPASS}

The recalibrated calibration plot of the predicted 2-year risk from the SMART-REACH model versus the observed 2-year risk of CVD in the COMPASS study population is shown in Figure 1a. C-statistics were $0.62(95 \% \mathrm{Cl} 0.61-0.64)$ for recurrent vascular events and 0.66 (95\% Cl 0.63-0.68) for non-CVD mortality risk.

\section{Development and validation of the major bleeding risk model}

The coefficients and subdistribution hazard ratios of the major bleeding and non-bleeding mortality models, age-specific baseline survivals and calculation formulas of the models are presented in Supplementary Table 2-4. No interaction terms with age were included in the functions as the proportional hazard assumptions were met. Quadratic terms for SBP, total cholesterol, and creatinine were included in the non-bleeding mortality model.

Figure $1 \mathrm{~b}$ shows good agreement between the predicted 2-year risk for major bleeding and mortality and the observed 2-year risk in the development dataset. Discrimination of the estimated 2-year bleeding risk was assessed, with a c-statistic of $0.69(95 \% \mathrm{Cl} 0.66$ $0.71)$, and the c-statistic of the 2 -year non-bleeding mortality risk was $0.71(95 \% \mathrm{Cl} 0.69$ 0.73). Figure 1c shows good agreement between the predicted and observed 10-year risk for bleeding and non-bleeding mortality after recalibration to account for differences in baseline risk in the SMART study population. Discrimination for major-bleeding free survival was assessed, with a c-statistic of $0.69(95 \% \mathrm{Cl} 0.67-0.70)$ in SMART.

\section{Individual lifetime estimates and treatment effects}

Figure 2 illustrates the use of the SMART-REACH and major bleeding risk model to estimate lifetime estimates of benefit and harm from rivaroxaban in a patient example.

The distribution of lifetime benefit in terms of months of lifetime gained without MACE and lifetime harm in terms of months lost without major bleeding with rivaroxaban added to aspirin in the combined study populations of the COMPASS and SMART studies is 


\begin{tabular}{|c|c|c|}
\hline & COMPASS study & SMART cohort \\
\hline & $n=27,390$ & $n=8,139$ \\
\hline Male sex & $21,371(78 \%)$ & $6,002(74 \%)$ \\
\hline Age (years) & $68 \pm 8$ & $60 \pm 10$ \\
\hline Current smoker & $5,867(21 \%)$ & 3,847 (47\%) \\
\hline \multicolumn{3}{|l|}{ Race } \\
\hline White & $17,023(62 \%)$ & N/A \\
\hline Black & $262(1 \%)$ & N/A \\
\hline Asian & $4,268(16 \%)$ & N/A \\
\hline Other & $5,837(21 \%)$ & N/A \\
\hline Systolic blood pressure (mmHg) & $139 \pm 18$ & $139 \pm 21$ \\
\hline Body mass index (kg/m2) & $28.3 \pm 4.7$ & $26.9 \pm 4.0$ \\
\hline \multicolumn{3}{|l|}{ Laboratory values } \\
\hline Total cholesterol (mmol/l) & $4.2 \pm 1.1$ & $4.8 \pm 1.2$ \\
\hline Creatinine $(\mu \mathrm{mol} / \mathrm{l})$ & $90 \pm 25$ & $92 \pm 36$ \\
\hline Estimated GFR (ml/min/1.73m2) & $74 \pm 18$ & $77 \pm 18$ \\
\hline \multicolumn{3}{|l|}{ Medical history } \\
\hline Coronary artery disease & 24,824 (91\%) & 4,939 (61\%) \\
\hline Peripheral artery disease & $7,470(27 \%)$ & $1,455(18 \%)$ \\
\hline Cerebrovascular disease & $1,032(4 \%)$ & 2,462 (30\%) \\
\hline \multicolumn{3}{|l|}{ No. of vascular bed disease locations } \\
\hline One & 21,186 (77\%) & 6,897 (85\%) \\
\hline Two & $4,800(18 \%)$ & $1,081(13 \%)$ \\
\hline Three & $1,404(5 \%)$ & $161(2 \%)$ \\
\hline Atrial fibrillation & $N A^{a}$ & $101(1 \%)^{b}$ \\
\hline Congestive heart failure & $5,902(22 \%)$ & $N A^{c}$ \\
\hline Diabetes mellitus & $10,340(38 \%)$ & $1,415(17 \%)$ \\
\hline Hepatic disease & $354(1.3 \%)$ & NA \\
\hline History of bleeding requiring transfusion & $723(2.6 \%)$ & NA \\
\hline
\end{tabular}

All data in $n(\%)$ or mean \pm standard deviation

GFR $=$ glomerular filtration rate (calculated with Chronic Kidney Disease Epidemiology Collaboration formula); $N A=$ not available.

a Patients requiring anticoagulation were excluded

b Only atrial fibrillation at baseline; history of atrial fibrillation not available

c Information not available 
Figure 1. (a) Predicted-versus-observed 2-year risk of CVD and all-cause mortality (SMARTREACH model) in the COMPASS trial; (b) Predicted-versus-observed 2-year risk of major bleeding and all-cause mortality in the COMPASS trial; (c) Predicted-versus-observed 10-year risk of major bleeding and all-cause mortality in the SMART cohort.
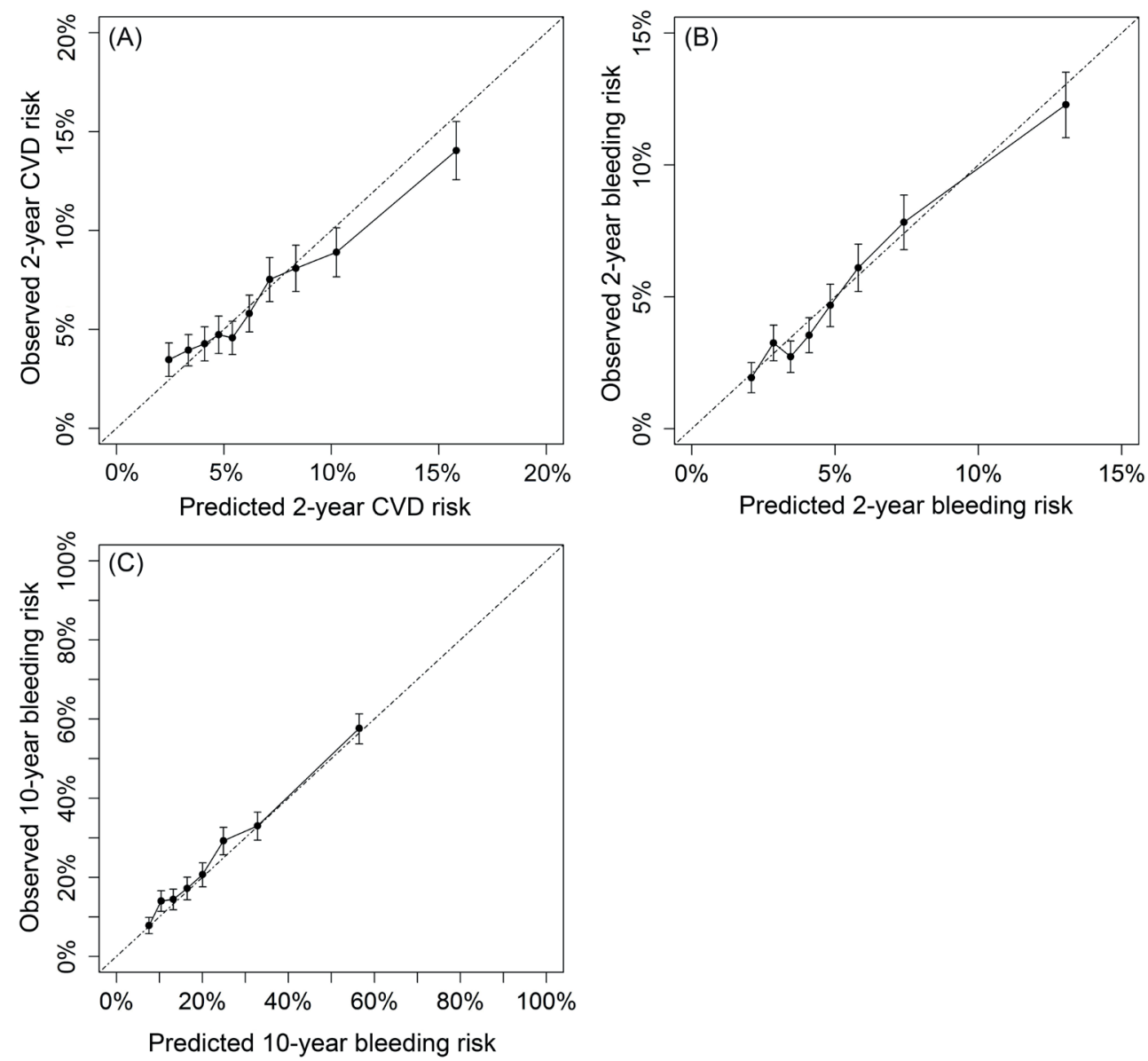

shown in Figure 3. The median lifetime benefit is 16 months (range 1-48 months) without MACE; the median lifetime harm is 2 months (range 0-20 months) without major bleeding. Figure 4 shows the balance between the individual absolute benefit and harm from adding rivaroxaban to aspirin in 20 groups ordered by increasing net benefit (defined as individual lifetime benefit minus lifetime major bleeding risk). For most of the patients, lifetime benefit in terms of CVD-free life expectancy is higher than lifetime harm. Supplementary Figure 1 shows the distribution of 10-year risks and 10-year absolute risk reductions with rivaroxaban for MACE and major bleeding respectively. 
Figure 2. Patient example.

\section{Cardiovascular disease}
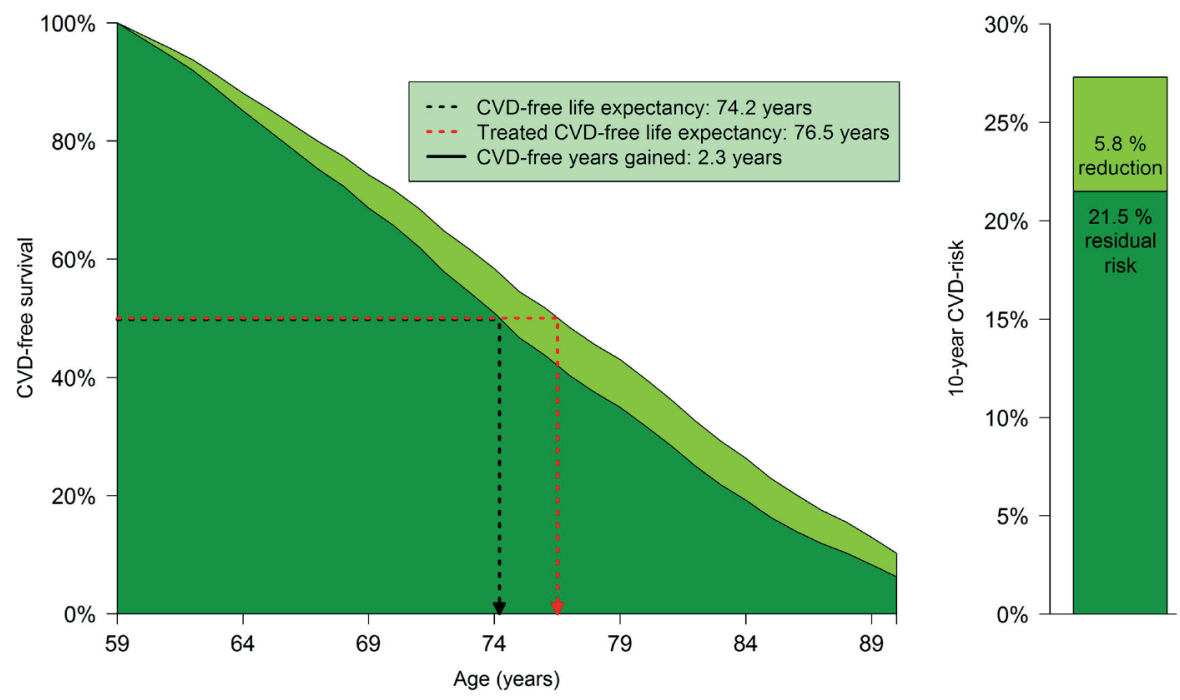

Major bleeding
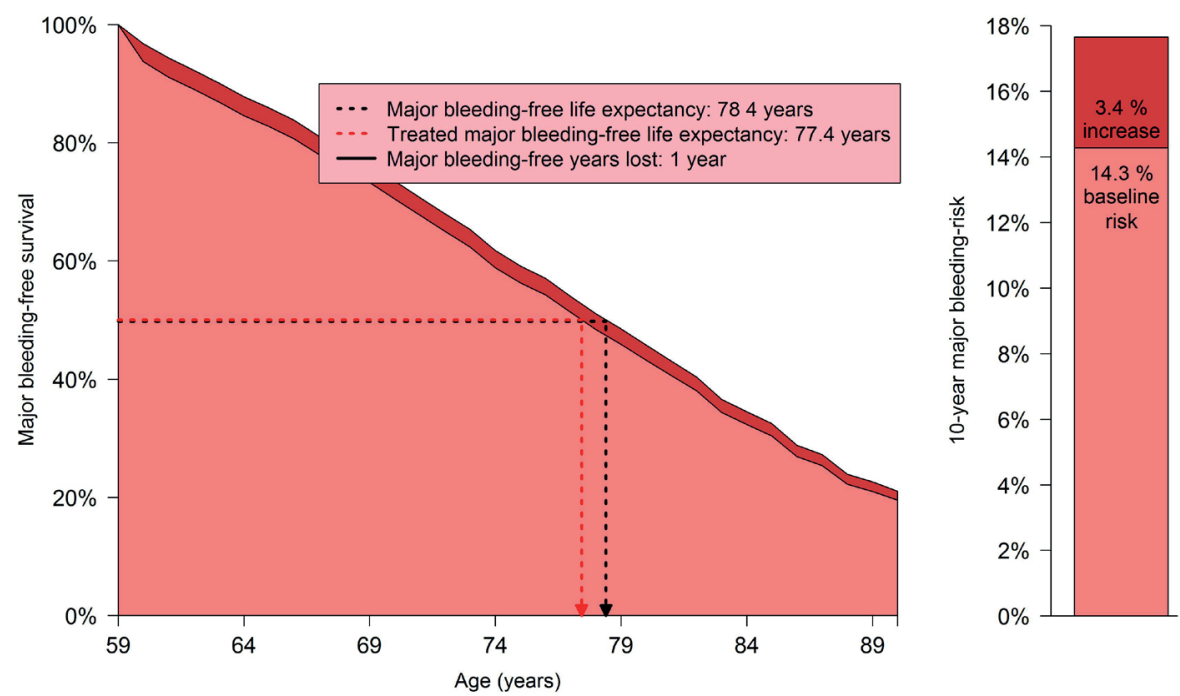

Patient characteristics: 59 year-old female from Canada; not a current smoker; systolic blood pressure $145 \mathrm{mmHg}$; total cholesterol $4.2 \mathrm{mmol} / \mathrm{L}$; creatinine $90 \mu \mathrm{mol} / \mathrm{L}$; history of coronary artery disease and peripheral artery disease; does not have diabetes mellitus, atrial fibrillation or congestive heart failure. The dark-green survival curve is the current estimated survival without recurrent CVD. The light-green area reflects the increase in estimated survival when adding low-dose rivaroxaban to aspirin (treatment benefit). The dark-red plus light-red areas of the lower survival curve represent the estimated survival without major bleeding. The light-red area represents the estimated decrease in survival without major bleeding when adding rivaroxaban to aspirin (treatment harm). The bar charts show the absolute 10-year risk and 10-year risk increase or decrease, respectively, of adding rivaroxaban to aspirin. 
Figure 3. Distribution of (a) lifetime benefit (months gained without recurrent myocardial infarction or stroke); (b) lifetime harm (months lost without major bleeding).
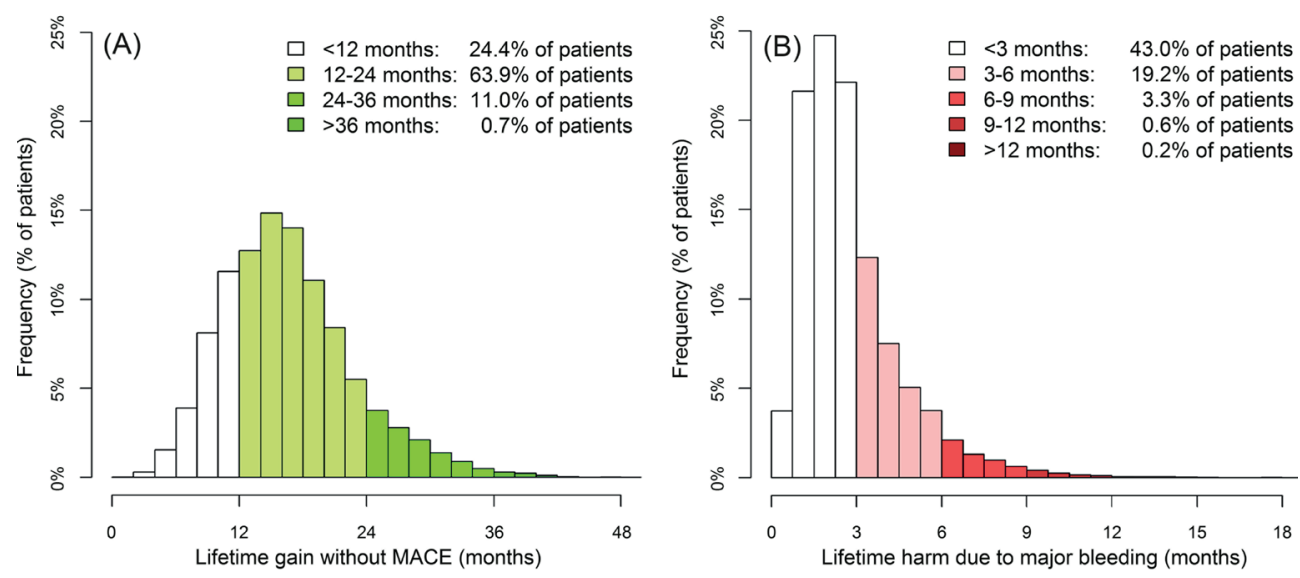

Figure 4. Individual lifetime benefit and associated lifetime harm from adding rivaroxaban to aspirin.

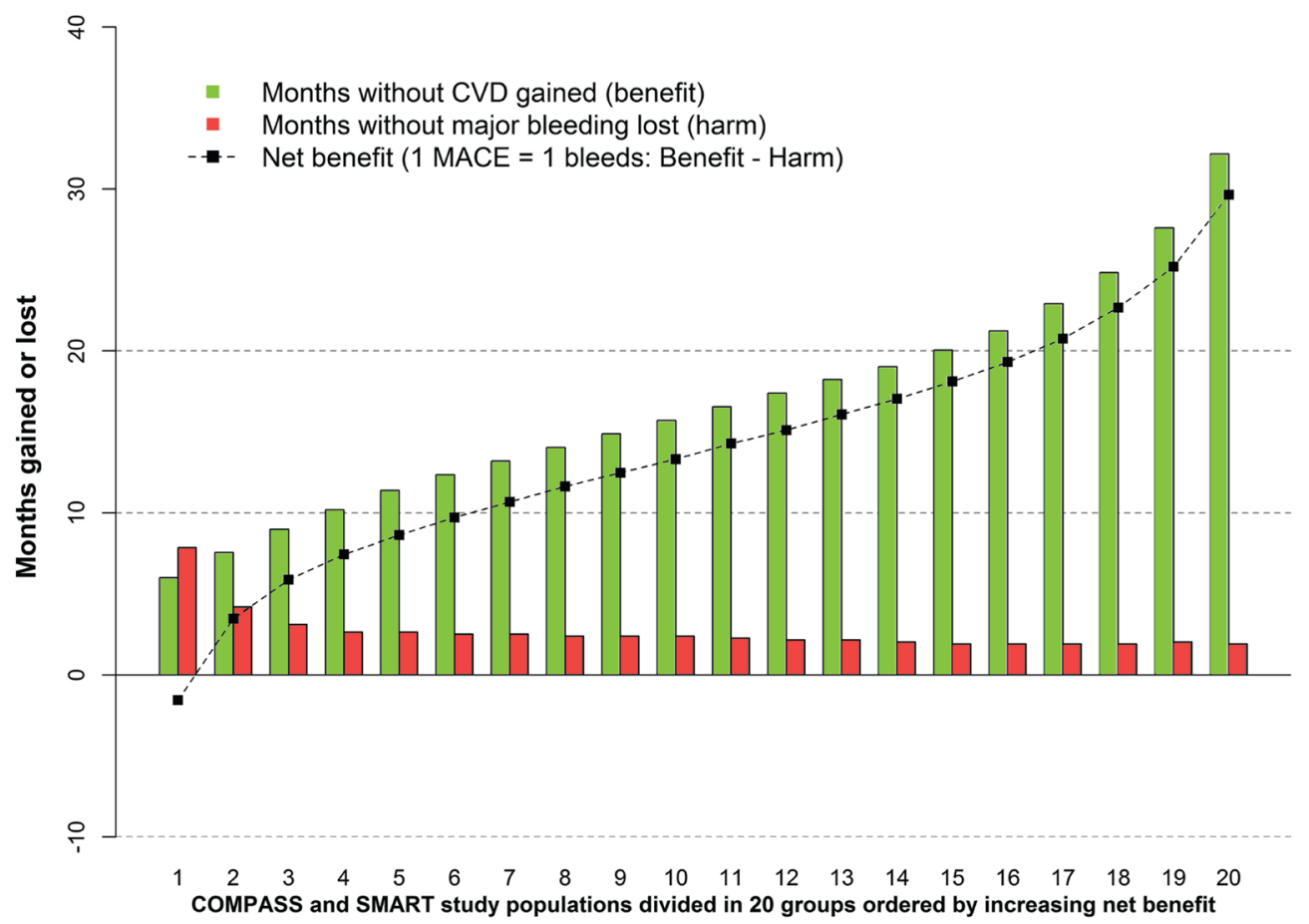




\section{Discussion}

In the current study, it is shown that individual lifetime benefit and lifetime risk of major bleeding from adding low-dose rivaroxaban to aspirin can be predicted in patients with stable cardiovascular disease. Lifetime benefit predictions were based on the externally validated SMART-REACH score, and major bleeding risk was based on the newly developed major bleeding risk score. These estimations can be made with simple, readily available patient characteristics. This enables the identification of patients who are likely to have long-term net benefit from rivaroxaban added to aspirin, in terms of additional CVD-free life expectancy, while having low risks of major bleeding.

An important clinical dilemma in the initiation of any antithrombotic is that the relative risk reduction of MACE is accompanied by a relative risk increase of bleeding. Because this study presents estimations for both lifetime treatment gain and treatment harm in terms of major bleeding, it aids in weighing the benefits versus harms when discussing with an individual patient with stable CVD whether or not to add rivaroxaban to the treatment strategy. Figure 4 shows that in the large majority of the COMPASS study population, lifetime benefit exceeds lifetime harm. However, there is a large inter-individual variation in what patients and physicians consider a meaningful lifetime benefit for preventive medication.22 Preconceived notions of treatment benefits and possible adverse effects of preventive treatment also influence these expectations. Furthermore, what is deemed acceptable benefit and harm from treatment is interdependent, as patients who have a higher benefit, might accept a higher risk of harm. Therefore, the data from the present study form the basis of shared decision making in clinical practice. Using individual lifetime estimates of treatment effects, a doctor and patient can discuss whether the estimated lifetime benefit, in terms of cardiovascular disease-free life, of adding rivaroxaban to aspirin is worthwhile, by weighing the benefit against potential burden of taking an extra pill twice daily, costs, and the risk of potential side effects, including bleeding. What is considered a meaningful balance between benefit and potential harms and disadvantages of preventive therapy may differ between patients. By shared decision making, patients are better informed and more involved in the process of making important decisions on life-long treatments which may lead in better treatment adherence as treatment decisions are tailored to their needs.

An advantage of the statistical methods of these models is that they can also be used to calculate 10-year risk estimations (as shown in Figure 2). As physicians are not yet widely familiar with the use of lifetime estimations, these absolute risk estimations might enable easier adoption of lifetime models, as they can be shown next to, and compared to, lifetime predictions in an online calculator. This can aid in a "transitional phase" in going from traditional risk models to the adoption of lifetime risk models. 
The lifetime scores presented in this study offer functionality superior to "traditional" risk scores which estimate absolute risks of CVD or major bleeding during a limited period, usually 5 to 10 years. Lifetime treatment effects can be expressed in both months of CVD free lifetime gained or lifetime lost with treatment and are intuitive to understand for both patients and physicians, facilitating doctor-patient communication and thus aiding shared treatment decision-making. Lifetime prediction has the potential to shift the focus from treatment of patients with high absolute risks, often older patients, to treatment of patients with the largest possible lifetime treatment gain. This strategy might lead to initiation of preventive medication at a younger age and presumably taken lifelong.

An important strength of this study is that in developing the models, competing risks are taken into account. Often, traditional risk scores do not take competing (i.e. non-CVD or non-bleeding respectively) mortality into account, which results in overestimation of risks in these patients in traditional risk models, and thus in overestimation of treatment effects. As treatment decisions are dependent on accurate predictions, this might have important implications for clinical practice. Secondly, due to the methodology using left-truncation, i.e. age as underlying time-function, estimations of these models are not limited by follow-up time in the derivation cohort. This means that despite limited followup time in the COMPASS trial, this study can be used for long-term estimations. Thirdly, the predictions in this model can be applied directly in clinical practice (www.U-Prevent. com). Finally, this study uses large study populations from a clinical trial with diverse geographical backgrounds, and from an observational cohort.

Some limitations of the study should also be considered. Validation could only be performed for 2-year predictions in COMPASS due to limited follow-up time in the study. External validation of 10 -year predictions in the SMART cohort, however, showed good calibration. Although remaining life expectancy, especially in younger patients, might be longer than 10 years, previous studies have shown that lifetime estimates based on the methods employed in this study appear to be reliable for predictions of up to at least 17 years, which is long enough for the purpose of making treatment decisions. ${ }^{12}$ C-statistics for discrimination of both models are moderate, comparable to other risk models in patients with established cardiovascular disease.15,17,23-26 External validation of 10-year predictions in the SMART cohort, however, showed good calibration. As reliability of the predicted probabilities influence treatment decisions, calibration may be a clinically more relevant metric than discrimination for the purpose of clinical decision making. ${ }^{27}$

Furthermore, the treatment effect estimations of rivaroxaban are based on studies with relatively short follow-up time, but projected for lifetime estimates. Although no data on long-term effects of rivaroxaban are yet available, for now there are no reasons to assume changes in efficacy or safety over time. Studies with long follow-up are needed. Furthermore, it should be acknowledged that non-compliance in the COMPASS trial 
may have affected the trial results. The estimated treatment effects in the current study approach the maximum attainable treatment effect with therapy. However, due to nonadherence in the trial, it is possible these predictions are underestimations of the true maximum attainable treatment effects with perfect therapy adherence.

Another limitation in that not all baseline predictors were available in both studies. For the SMART-REACH model, atrial fibrillation was not available in the COMPASS trial. For the major bleeding risk model, the history of heart failure and history of bleeding requiring transfusion were not available in the SMART cohort. Assuming that none of the participants in these studies had a history of atrial fibrillation and congestive heart failure or bleeding requiring transfusion, respectively, might lead to underestimation of the predictive value of the model. Additionally, there was some underestimation of the predicted risk of CVD and mortality combined in the patients in the lowest decile of risk, and some overestimation in the highest decile of risk in the COMPASS study population. In clinical practice, however, this may not be of clinical relevance as this most likely does not result in misclassification or incorrect treatment decisions. Finally, the baseline risk for both cardiovascular and non-cardiovascular mortality was vastly different in the COMPASS trial compared to the study population in which the SMART-REACH model was derived due to differences in patient populations, for example the higher percentage of current smokers and patients with cerebrovascular disease at baseline in the SMART cohort. Additionally, this may be due to a healthy trial participant effect, as the SMART-REACH model was derived in cohorts, which better reflects real-life patients than a trial. ${ }^{28,29}$ As the major bleeding risk model was derived in a trial, additional validation with recalibration for baseline risk in real-life situations or cohorts should be considered.

\section{Conclusion}

Lifetime treatment effects from adding rivaroxaban to aspirin in individual patients with stable CVD can be estimated using readily available patient characteristics. There is a wide distribution in lifetime gain and harm from adding rivaroxaban to aspirin in patients with stable CVD. Using this lifetime model, benefits and bleeding risk can be weighed for each individual patient, facilitating informed treatment decisions in clinical practice. 


\section{References}

1. Kaasenbrood L, Boekholdt SM, van der Graaf Y, Ray KK, Peters RJG, Kastelein JJP, et al. Distribution of Estimated 10-Year Risk of Recurrent Vascular Events and Residual Risk in a Secondary Prevention Population. Circulation. 2016;134(19):1419-29.

2. Smith SCJ, Benjamin EJ, Bonow RO, Braun LT, Creager MA, Franklin BA, et al. AHA/ACCF Secondary Prevention and Risk Reduction Therapy for Patients with Coronary and other Atherosclerotic Vascular Disease: 2011 update: a guideline from the American Heart Association and American College of Cardiology Foundation. Circulation. 2011;124(22):2458-73.

3. Piepoli MF, Hoes AW, Agewall S, Albus C, Brotons C, Catapano AL, et al. 2016 European guidelines on cardiovascular disease prevention in clinical practice: the Sixth Joint Task Force of the European Society of Cardiology and Other Societies on Cardiovascular Disease Prevention in Clinical Practice. Eur Heart J. 2016;37(29):2315-81.

4. Eikelboom JW, Connolly SJ, Bosch J, Dagenais GR, Hart RG, Shestakovska O, et al. Rivaroxaban with or without Aspirin in Stable Cardiovascular Disease. N Engl J Med. 2017;377(14):1319-30.

5. Kent DM, Steyerberg E, van Klaveren D. Personalized evidence based medicine: predictive approaches to heterogeneous treatment effects. BMJ. 2018;363:k4245.

6. Bosch J, Eikelboom JW, Connolly SJ, Bruns NC, Lanius V, Yan F, et al. Rationale, Design and Baseline Characteristics of Participants in the Cardiovascular Outcomes for People Using Anticoagulation Strategies (COMPASS) Trial. Can J Cardiol. 2017;33:1027-35.

7. Simons PC, Algra A, van de Laak MF, Grobbee DE, van der Graaf Y. Second manifestations of ARTerial disease (SMART) study: rationale and design. Eur J Endocrinol. 1999;15(9):773-81.

8. Schulman S, Kearon; Subcommittee on Control of Anticoagulation of the Scientific and Standardization Committee of the International Society on Thrombosis and Haemostasis. Definition of major bleeding in clinical investigations of antihemostatic medicinal products in non-surgical patients. J Thromb Haemost. 2005;3(4):692-4.

9. Kaasenbrood L, Bhatt DL, Dorresteijn JA, Wilson PW, D'Agostino RB, Massaron JM, et al. Estimated life-expectancy without recurrent cardiovascular events in patients with vascular disease: the REACH-SMART model. J Am Hear. 2018;7:e009217.

10. Geskus RB. Cause-specific cumulative incidence estimation and the fine and gray model under both left truncation and right censoring. Biometrics. 2011;67(1):39-49.

11. Dorresteijn JAN, Visseren FLJ, Ridker PM, Wassink AMJ, Paynter NP, Steyerberg EW, et al. Estimating treatment effects for individual patients based on the results of randomised clinical trials. BMJ. 2011;343:d5888.

12. Dorresteijn JAN, Kaasenbrood L, Cook NR, van Kruijsdijk RCM, van der Graaf Y, Visseren FLJ, et al. How to translate clinical trial results into gain in healthy life expectancy for individual patients. BMJ. 2016;352:i1548.

13. Kaasenbrood L, Ray K, Boekholdt SM, Smulders Y, Kastelein JJ, van der Graaf Y, et al. Estimated individual lifetime benefit from PCSK9 -inhibition in statin-treated patients with coronary artery 
disease. Heart. 2018;104(20):1699-1705.

14. Hippisley-Cox J, Coupland C, Robson J, Brindle P. Derivation, validation, and evaluation of a new QRISK model to estimate lifetime risk of cardiovascular disease: cohort study using QResearch database. BMJ. 2010;341.

15. Costa F, van Klaveren D, James $S$, Heg D, Raber L, Feres F, et al. Derivation and validation of the predicting bleeding complications in patients undergoing stent implantation and subsequent dual antiplatelet therapy (PRECISE-DAPT) score: a pooled analysis of individual-patient datasets from clinical trials. Lancet. 2017;389(10073):1025-34.

16. Hilkens NA, Algra A, Diener H-C, Reitsma JB, Bath PM, Csiba L, et al. Predicting major bleeding in patients with noncardioembolic stroke on antiplatelets: S2TOP-BLEED. Neurology. 2017;89(9):936-43.

17. Ducrocq G, Wallace JS, Baron G, Ravaud P, Alberts MJ, Wilson PWF, et al. Risk score to predict serious bleeding in stable outpatients with or at risk of atherothrombosis. Eur Heart J. 2010;31(10):1257-65.

18. Steyerberg EW. Clinical prediction models: a practical approach to development, validation and updating. New York, USA: Springer; 2009.

19. Lloyd-Jones DM, Huffman MD, Karmali KN, Sanghavi DM, Wright JS, Pelser C, et al. Estimating Longitudinal Risks and Benefits From Cardiovascular Preventive Therapies Among Medicare Patients: The Million Hearts Longitudinal ASCVD Risk Assessment Tool: A Special Report From the American Heart Association and American College of Cardiolog. J Am Coll Cardiol. 2017;69(12):1617-36.

20. Berkelmans GF, Gudbjörnsdottir S, Visseren FL, Wild SH, Franzen S, Chalmers J, et al. Prediction of individual life-years gained without cardiovascular events from lipid, blood pressure, glucose, and aspirin treatment based on data of more than 500000 patients with Type 2 diabetes mellitus. Eur Heart J. 2019; [Epub ahead of print].

21. Connolly SJ, Eikelboom JW, Bosch J, Dagenais G, Dyal L, Lanas F, et al. Rivaroxaban with or without aspirin in patients with stable coronary artery disease: an international, randomised, double-blind, placebo-controlled trial. Lancet. 2018;391(10117):205-18.

22. Jaspers NEM, Visseren FLJ, Numans ME, Smulders YM, van Loenen Martinet FA, van der Graaf $Y$, et al. Variation in minimum desired cardiovascular disease-free longevity benefit from statin and antihypertensive medications: a cross-sectional study of patient and primary care physician perspectives. BMJ Open. 2018;8(5):e021309.

23. Huang D, Cheng Y-Y, Wong Y-TA, Yung S-YA, Tam C-CF, Chan K-WK, et al. Thrombolysis in Myocardial Infarction Risk Score for Secondary Prevention of Recurrent Cardiovascular Events in a Real-World Cohort of Post-Acute Myocardial Infarction Patients. Circ J. 2019;83(4):809-17.

24. Yejin $M, H . B S, D . B L, L . B D, E . B W, P . B M$, et al. International Validation of the Thrombolysis in Myocardial Infarction (TIMI) Risk Score for Secondary Prevention in Post-MI Patients: A Collaborative Analysis of the Chronic Kidney Disease Prognosis Consortium and the Risk Validation Scientific Committee. J Am Heart Assoc. 2018;7(14):e008426. 
25. Wilson PWF, D'Agostino R, Bhatt DL, Eagle K, Pencina MJ, Smith SC, et al. An International Model to Predict Recurrent Cardiovascular Disease. Am J Med. 2012;125(7):695-703.e1.

26. Dorresteijn JAN, Visseren FLJ, Wassink AMJ, Gondrie MJA, Steyerberg EW, Ridker PM, et al. Development and validation of a prediction rule for recurrent vascular events based on a cohort study of patients with arterial disease: the SMART risk score. Heart. 2013;99(12):866-72.

27. Cook NR. Use and Misuse of the Receiver Operating Characteristic Curve in Risk Prediction. Circulation. 2007;115(7):928-35.

28. Timmis A, Rapsomaniki E, Chung SC, Pujades-Rodriguez M, Moayyeri A, Stogiannis D, et al. Prolonged dual antiplatelet therapy in stable coronary disease: comparative observational study of benefits and harms in unselected versus trial populations. BMJ. 2016;353:i3163.

29. Maasland L, van Oostenbrugge RJ, Franke CF, Scholte Op Reimer WJM, Koudstaal PJ, Dippel DWJ. Patients enrolled in large randomized clinical trials of antiplatelet treatment for prevention after transient ischemic attack or ischemic stroke are not representative of patients in clinical practice: the Netherlands Stroke Survey. Stroke. 2009;40(8):2662-8. 


\section{Supplementary material}

\section{Supplemental methods \\ SMART-REACH}

The SMART-REACH risk model is a competing-risk adjusted Fine \& Gray model for lifetime predictions for major cardiovascular events and non-cardiovascular mortality in patients with clinically manifest vascular disease, derived in the REduction of Atherothrombosis for Continued Health (REACH) registry WesternEurope. It was externally validated in the SMART cohort study and REACH registry North-America, with C-statistics of respectively 0.68 ( $95 \% \mathrm{Cl} 0.67$ to 0.70 ) and 0.67 (95\% Cl 0.66 to 0.68$)$, and calibration plots of predicted versus observed risk showed high agreement. It is based on the following predictors: sex, current smoking, diabetes mellitus, systolic blood pressure (SBP), total cholesterol, and creatinine, number of locations of cardiovascular disease (i.e., $C A D, C V D$, and PAD), history of atrial fibrillation, and history of congestive heart failure. The model can be used for estimation of both 10-year risk of cardiovascular events and life-expectancy without recurrent cardiovascular events. Additionally, it can be used to predict improvement in life-expectancy with several preventive therapies. ${ }^{1}$

These are the underlying formulas:

\section{Cardiovascular model}

1-year survival $=\left(\text { age-specific } 1 \text {-yr baseline survival }{ }^{*}\right)^{\wedge} \exp (A)$

$A=0.0720$ (if male) +0.4309 (if current smoker) +0.4357 (if diabetes mellitus) $-0.0281^{\star}$ systolic blood pressure (in $\mathrm{mmHg})+0.0001^{*}$ squared systolic blood pressure (in $\mathrm{mmHg}$ ) $-0.3671^{*}$ total cholesterol (in $\mathrm{mmol} / \mathrm{L}$ ) $+0.0356^{*}$ squared total cholesterol (in mmol/L) $+0.0061^{\star} \mathrm{creatinine}$ (in $\mu \mathrm{mol} / \mathrm{L}$ ) +0.3176 (if two locations of cardiovascular disease) $)^{\S} 0.2896$ (if three locations of cardiovascular disease) $)^{\S}+0.2143$ (if history of atrial fibrillation) +0.4447 (if history of congestive heart failure)

\section{Non-cardiovascular mortality model}

1-year survival $=\left(\text { age-specific } 1-y r \text { baseline survival }{ }^{*}\right)^{\wedge} \exp (B)$

$B=0.5986$ (if male) +4.2538 (if current smoker) $-0.0486^{*}$ age (if current smoker) +0.4065 (if

diabetes mellitus) $-0.0074 *$ systolic blood pressure (in $\mathrm{mmHg}$ ) $-0.0030 *$ total cholesterol (in $\mathrm{mmol} / \mathrm{L}$ )

- $0.0189^{*}$ creatinine (in $\mu \mathrm{mol} / \mathrm{L}$ ) $+0.0001^{*}$ squared creatinine (in $\mu \mathrm{mol} / \mathrm{L}$ ) +0.1442 (if two locations of

cardiovascular disease) $^{\S}+0.5694$ (if three locations of cardiovascular disease) $)^{\S}+0.3213$ (if history of atrial

fibrillation) +0.2061 (if history of congestive heart failure)

${ }^{*}$ Age-specific baseline survivals can be found in the supplemental material of the SMART-REACH manuscript ${ }^{1}$ $\S$ The coefficients for number of locations of cardiovascular disease (CAD, CVD, PAD) should not be added up. So, if the patient has two locations of cardiovascular disease, add 0.3176 to $A$ and 0.1442 to $B$; if the patient has three locations of cardiovascular disease, add 0.2896 to $A$ and 0.5694 to $B$. For patients similar to the Dutch (SMART) population: add -0.4246 to $A$ and 0.1232 to B. For North-American patients or patients similar to the North-American REACH population: add 0.1552 to $A$ and 0.4134 to $B$. 


\section{External validation and recalibration of the SMART-REACH model}

Using 2-year risk predictions, obtained using the methods described under the heading 'Predictions for individual persons' in the methods section of the main manuscript, calibration plots of the predictedversus-observed 2-year risks were made to assess calibration of the model in the control arm of the COMPASS study population. As the underlying event rate was lower in the COMPASS trial than in REACH and SMART, the ratio between expected and observed events (E/O ratio) in the COMPASS study population was used to update the models to the population of interest, stratified for the geographical region to account for regional differences in event rates (Western-Europe/Australia, Eastern Europe, North America, South America, Asia). Discrimination was assessed using the C-statistic (95\% confidence interval).

\section{Supplementary references}

1. Kaasenbrood L, Bhatt DL, Dorresteijn JA, Wilson PW, D'Agostino RB, Massaron JM, Graaf $Y$ van der, Cramer MJ, Kappelle LJ, Borst GJ de, Steg PG, Visseren FL. Estimated life-expectancy without recurrent cardiovascular events in patients with vascular disease: the REACH-SMART model. J Am Hear 2018;7:e009217. 


\section{Supplemental Results}

Supplemental Table 1. Inclusion and exclusion criteria of the COMPASS and SMART cohorts and definitions of outcome events.

(a) Inclusion criteria

\begin{tabular}{|c|c|c|}
\hline & SMART & COMPASS \\
\hline $\begin{array}{l}\text { Inclusion } \\
\text { criteria }\end{array}$ & $\begin{array}{l}\text { Patients aged } 40-79 \text { years with } \\
\text { documented } C A D, C V D \text {, or PAD }\end{array}$ & $\begin{array}{l}\text { Patients meeting the criteria for CAD and/or } \\
\text { PAD; aged }>65 \text {, or aged }<65 \text { with documented } \\
\text { atherosclerosis or revascularization involving } \\
\text { at least } 2 \text { vascular beds or with at least two } \\
\text { additional risk factors }\end{array}$ \\
\hline $\begin{array}{l}\text { Exclusion } \\
\text { criteria }\end{array}$ & $\begin{array}{l}\text {-Terminal malignancy } \\
\text {-Not independent in daily activities } \\
\text { (Rankin scale }>3 \text { ) } \\
\text {-Not sufficiently fluent in Dutch }\end{array}$ & $\begin{array}{l}\text { - High risk of bleeding } \\
\text { - Recent (<1 month) stroke; history of } \\
\text { hemorrhagic or lacunar stroke } \\
\text { - Severe heart failure (ejection fraction }<30 \% \text { or } \\
\text { NYHA class III or IV symptoms) } \\
\text { - eGFR }<15 \mathrm{ml} / \mathrm{min} \\
\text { - Need for dual antiplatelet therapy or oral } \\
\text { anticoagulant therapy } \\
\text { - Known non-cardiovascular disease associated } \\
\text { with a poor prognosis } \\
\text { - Any known hepatic disease associated with } \\
\text { coagulopathy }\end{array}$ \\
\hline
\end{tabular}

(b) Definitions of outcome events

\begin{tabular}{|c|c|c|}
\hline & SMART & COMPASS \\
\hline $\begin{array}{l}\text { Outcome } \\
\text { evaluation }\end{array}$ & $\begin{array}{l}\text { During follow-up, patients were asked } \\
\text { biannually to complete a standardized } \\
\text { questionnaire on hospital admissions } \\
\text { and outpatient clinic visits. If a vascular } \\
\text { event was reported, hospital discharge } \\
\text { letters and results of laboratory and } \\
\text { radiology examinations were collected. } \\
\text { Death was reported by relatives of the } \\
\text { participant, the general practitioner or } \\
\text { the treating specialist. All possible events } \\
\text { were independently evaluated by three } \\
\text { members of the endpoint committee, } \\
\text { comprising physicians from different } \\
\text { clinical departments. }\end{array}$ & $\begin{array}{l}\text { Participants were seen at } 1 \text { and } 6 \text { months } \\
\text { after randomization, and thereafter at } \\
6 \text {-month intervals to record outcomes } \\
\text { and adverse events. }\end{array}$ \\
\hline
\end{tabular}


(b) Definitions of outcome events (continued)

\begin{tabular}{|c|c|c|}
\hline & SMART & COMPASS \\
\hline $\begin{array}{l}\text { Myocardial } \\
\text { infarction }\end{array}$ & $\begin{array}{l}\text { Fatal and non-fatal myocardial infarction, } \\
\text { characterized by at least two of the } \\
\text { following criteria: } \\
\text { 1. Chest pain for at least } 20 \text { minutes not } \\
\text { disappearing after administration of } \\
\text { nitrates } \\
\text { 2. ST-elevation }>1 \mathrm{~mm} \text { in two following } \\
\text { leads or a left bundle branch block on the } \\
\text { ECG * } \\
\text { 3. CK elevation of at least two times the } \\
\text { normal value of CK and an MB-fraction } \\
>5 \% \text { of the total CK }\end{array}$ & $\begin{array}{l}\text { Fatal and non-fatal myocardial infarction, } \\
\text { with } \\
\text { 1. Cardiac ischemic symptoms, and/or } \\
\text { 2. ECG or imaging changes consistent } \\
\text { with MI (e.g. ST-segment changes, } \\
\text { development of new left bundle } \\
\text { branch block, new diagnostic Q waves, } \\
\text { identification of intracoronary thrombus } \\
\text { by angiography), and/or } \\
\text { 3. Increased cardiac markers (e.g. troponin } \\
>5 \times \text { 99th percentile) }\end{array}$ \\
\hline Stroke & $\begin{array}{l}\text { Relevant clinical features which have } \\
\text { caused an increase in handicap of at } \\
\text { least one grade on the modified Rankin } \\
\text { scale, accompanied by a fresh infarct on a } \\
\text { repeat CT scan. }\end{array}$ & $\begin{array}{l}\text { Presence of acute focal neurological } \\
\text { deficit thought to be of vascular origin } \\
\text { with signs and symptoms lasting } \geq 24 \\
\text { hours or to time of death. The stroke } \\
\text { definition included the main categories } \\
\text { definite ischemic stroke, definite } \\
\text { hemorrhagic stroke, and uncertain or } \\
\text { unknown stroke }\end{array}$ \\
\hline $\begin{array}{l}\text { Cardiovascular } \\
\text { death }\end{array}$ & $\begin{array}{l}\text {-Sudden death: unexpected cardiac } \\
\text { death occurring within } 1 \text { hour after onset } \\
\text { of symptoms or within } 24 \text { hours given } \\
\text { convincing circumstantial evidence } \\
\text {-Death from ischemic stroke } \\
\text {-Death from congestive heart failure } \\
\text {-Death from myocardial infarction } \\
\text {-Death from rupture of abdominal aortic } \\
\text { aneurysm } \\
\text {-Vascular death from other cause, i.e. } \\
\text { sepsis following stent placement }\end{array}$ & $\begin{array}{l}\text { Death for which a definite non- } \\
C V \text { cause (e.g. cancer) had not been } \\
\text { identified }\end{array}$ \\
\hline Bleeding & $\begin{array}{l}\text { Any intracranial bleeding, fatal bleeding } \\
\text { and any bleeding complication requiring } \\
\text { hospitalization }\end{array}$ & $\begin{array}{l}\text { ISTH: Fatal bleeding, bleeding in a critical } \\
\text { organ (incl intracranial), bleeding causes } \\
\text { a fall in } \mathrm{Hb}>20 \mathrm{~g} / \mathrm{L} \text { or leads to transfusion } \\
\text { or }>=2 \text { units of blood cells }\end{array}$ \\
\hline
\end{tabular}


Supplemental Table 2. Coefficients and subdistribution hazard ratios of the major bleeding risk model

\begin{tabular}{|c|c|c|c|c|}
\hline \multirow[b]{2}{*}{ Male sex } & \multicolumn{2}{|c|}{$\begin{array}{l}\text { Model 1: bleeding } \\
\text { CoefficientsHR (95\% CI) }\end{array}$} & \multicolumn{2}{|c|}{$\begin{array}{l}\text { Model 2: competing event } \\
\text { Coefficient sHR ( } 95 \% \text { CI) }\end{array}$} \\
\hline & -0.1608 & $0.85(0.68-1.07)$ & 0.1334 & $1.14(0.97-1.35)$ \\
\hline \multicolumn{5}{|l|}{ Ethnicity } \\
\hline White or Caucasian & 0 (ref) & 1 (ref) & 0 (ref) & 1 (ref) \\
\hline Black or African American & -0.6676 & $0.51(0.16-1.62)$ & 0.3774 & $1.46(0.83-2.57)$ \\
\hline Asian & 0.2946 & $1.34(0.92-1.96)$ & -0.3851 & $0.68(0.51-0.91)$ \\
\hline Other & 0.4400 & $1.55(0.99-2.45)$ & -0.1272 & $0.88(0.67-1.16)$ \\
\hline \multicolumn{5}{|l|}{ Geographical region } \\
\hline North America & 0 (ref) & 1 (ref) & 0 (ref) & 1 (ref) \\
\hline West-Europe & -0.3072 & $0.74(0.57-0.95)$ & -0.0489 & $0.95(0.75-1.21)$ \\
\hline East-Europe & -0.7425 & $0.48(0.34-0.67)$ & 0.2058 & $1.23(0.95-1.58)$ \\
\hline South America & -1.3442 & $0.26(0.16-0.43)$ & 0.7312 & $2.08(1.52-2.83)$ \\
\hline Other & -0.2817 & $0.75(0.52-1.10)$ & 0.4966 & $1.64(1.22-2.21)$ \\
\hline Current smoking & 0.3392 & $1.40(1.11-1.78)$ & 0.5637 & $1.76(1.49-2.07)$ \\
\hline Systolic blood pressure (per 10 mmHg) & 0.0460 & $1.00(1.00-1.01)$ & -0.4920 & $N / E^{*}$ \\
\hline Systolic blood pressure squared (per $10 \mathrm{mmHg}$ ) & N/A & $\mathrm{N} / \mathrm{A}$ & 0.0020 & $N / E^{*}$ \\
\hline \multicolumn{5}{|l|}{ Number of cardiovascular beds } \\
\hline One & 0 (ref) & 1 (ref) & 0 (ref) & 1 (ref) \\
\hline Two & 0.4885 & $1.63(1.32-2.02)$ & 0.3743 & $1.45(1.25-1.69)$ \\
\hline Three & 0.2981 & $1.35(0.93-1.95)$ & 0.3684 & $1.45(1.13-1.85)$ \\
\hline Congestive heart failure & 0.2437 & $1.28(1.01-1.62)$ & 0.4634 & $1.59(1.37-1.85)$ \\
\hline Diabetes mellitus & 0.1026 & $1.11(0.92-1.33)$ & 0.4275 & $1.53(1.35-1.75)$ \\
\hline History of bleeding requiring transfusion & 0.6963 & $2.01(1.41-2.86)$ & 0.0146 & $1.01(0.70-1.47)$ \\
\hline Total cholesterol (per mmol/L) & -0.0913 & $0.91(0.83-1.01)$ & -0.3286 & $N / E^{*}$ \\
\hline Total cholesterol squared (per mmol/L) & N/A & $\mathrm{N} / \mathrm{A}$ & 0.0437 & $N / E^{*}$ \\
\hline Creatinine (per 10 mol/L) & 0.0780 & $1.01(1.00-1.01)$ & -0.1630 & $N / E^{*}$ \\
\hline Creatinine squared (per $10 \mu \mathrm{mol} / \mathrm{L}$ ) & N/A & $\mathrm{N} / \mathrm{A}$ & 0.0010 & $N / E^{*}$ \\
\hline $\begin{array}{l}\text { * Model } 2 \text { contains squared terms } \\
\text { and creatinine, and an interaction } b \\
\text { only coefficients were provided as }\end{array}$ & $\begin{array}{l}\text { for } S \\
\text { between } \\
\text { the } H F\end{array}$ & $\begin{array}{l}\text { stolic blood p } \\
\text { creatinine and } \\
\text { cannot be }\end{array}$ & $\begin{array}{l}\text { oressure, } \\
\text { age. } \\
\text { interpret }\end{array}$ & $\begin{array}{l}\text { otal cholesterol, } \\
r \text { these terms } \\
\text { independently. }\end{array}$ \\
\hline
\end{tabular}


Supplemental Table 3. Age-specific baseline survivals for the major bleeding risk model

\begin{tabular}{|c|c|c|}
\hline Age & $\begin{array}{c}\text { 1-year survival free } \\
\text { of bleeding* }\end{array}$ & $\begin{array}{c}\text { 1-year survival for } \\
\text { competing mortality }^{* *}\end{array}$ \\
\hline 45 & 1.0000 & 0.4042 \\
\hline 46 & 0.9976 & 0.7489 \\
\hline 47 & 1.0000 & 0.7048 \\
\hline 48 & 0.9953 & 0.6440 \\
\hline 49 & 0.9958 & 0.6995 \\
\hline 50 & 1.0000 & 0.6337 \\
\hline 51 & 1.0000 & 0.5667 \\
\hline 52 & 0.9979 & 0.7209 \\
\hline 53 & 1.0000 & 0.7940 \\
\hline 54 & 0.9984 & 0.7916 \\
\hline 55 & 0.9989 & 0.8221 \\
\hline 56 & 0.9990 & 0.5536 \\
\hline 57 & 0.9992 & 0.6253 \\
\hline 58 & 0.9987 & 0.5981 \\
\hline 59 & 0.9970 & 0.6238 \\
\hline 60 & 0.9978 & 0.6724 \\
\hline 61 & 0.9981 & 0.6853 \\
\hline 62 & 0.9986 & 0.5341 \\
\hline 63 & 0.9983 & 0.5064 \\
\hline 64 & 0.9991 & 0.4786 \\
\hline 65 & 0.9983 & 0.5363 \\
\hline 66 & 0.9972 & 0.5702 \\
\hline 67 & 0.9980 & 0.6074 \\
\hline 68 & 0.9973 & 0.5133 \\
\hline 69 & 0.9970 & 0.4859 \\
\hline 70 & 0.9969 & 0.4815 \\
\hline 71 & 0.9964 & 0.5664 \\
\hline 72 & 0.9966 & 0.5203 \\
\hline 73 & 0.9952 & 0.4056 \\
\hline 74 & 0.9969 & 0.3786 \\
\hline 75 & 0.9980 & 0.3374 \\
\hline 76 & 0.9958 & 0.3372 \\
\hline 77 & 0.9955 & 0.3585 \\
\hline 78 & 0.9959 & 0.3699 \\
\hline 79 & 0.9961 & 0.2489 \\
\hline 80 & 0.9959 & 0.2399 \\
\hline 81 & 0.9961 & 0.1808 \\
\hline 82 & 0.9935 & 0.0964 \\
\hline 83 & 0.9967 & 0.1840 \\
\hline 84 & 0.9969 & 0.1598 \\
\hline 85 & 0.9898 & 0.1512 \\
\hline 86 & 1.0000 & 0.0502 \\
\hline 87 & 0.9927 & 0.0282 \\
\hline 88 & 0.9961 & 0.2764 \\
\hline 89 & 0.9954 & 0.1729 \\
\hline
\end{tabular}




\section{Supplemental Table 4. Major bleeding risk model formulas}

\section{Major bleeding model}

1-year survival $=\left(\text { age-specific } 1 \text {-yr baseline survival }{ }^{*}\right)^{\wedge} \exp (A)$

$A=-0.1608$ (if male) -0.6676 (if African American) + 0.2946 (if Asian) +0.4400 (if other race (not Caucasian) $\left.^{\dagger}\right)+0.3392$ (if current smoker) +0.1026 (if diabetes mellitus) +0.0046 * systolic blood pressure (in $\mathrm{mmHg}$ ) $-0.0913^{*}$ total cholesterol (in mmol/L) + 0.0078*creatinine (in $\mu \mathrm{mol} / \mathrm{L}$ ) + 0.4885 (if two locations of cardiovascular disease) ${ }^{\S}+0.2981$ (if three locations of cardiovascular disease) ${ }^{\S}+0.6963$ (if history of bleeding requiring transfusion) +0.2437 (if history of congestive heart failure) -0.09584

\section{Mortality not due to major bleeding - model}

1-year survival $=\left(\text { age-specific } 1 \text {-yr baseline survival }{ }^{*}\right)^{\wedge} \exp (B)$ $B=0.1334$ (if male) + 0. 3774 (if African American) - 0.3851 (if Asian) - 0.1272 (if other race $\left.(\text { not Caucasian) })^{\dagger}\right)+0.5637$ (if current smoker) +0.4275 (if diabetes mellitus) $-0.0492^{*}$ systolic blood pressure (in $\mathrm{mmHg}$ ) $+0.0002^{*}$ squared systolic blood pressure (in $\mathrm{mmHg}$ ) $-0.3286^{*}$ total cholesterol (in mmol/L) $+0.0437^{\star}$ squared total cholesterol (in $\mathrm{mmol} / \mathrm{L}$ ) $-0.0163^{\star}$ creatinine (in $\mu \mathrm{mol} / \mathrm{L}$ ) $+0.0001^{\star}$ squared creatinine (in $\mu \mathrm{mol} / \mathrm{L}$ ) +0.3743 (if two locations of cardiovascular disease) $)^{\S}+0.3684$ (if three locations of cardiovascular disease) ${ }^{\S}+0.0146$ (if history of bleeding requiring transfusion) +0.4634 (if history of congestive heart failure) +0.3674

$¥$ Age-specific baseline survivals are shown in Supplemental Table 2 for both models

+ As Caucasian is used as the reference, no coefficient should be added for Caucasian patients.

$\S$ The coefficients for number of locations of cardiovascular disease (CAD, CVD, PAD) should not be added up.

For patients from Western Europe: add -0.3072 to $A$ and -0.0489 to B. For patients from Eastern Europe: add -0.7425 to $A$ and 0.2058 to B. For patients from South America: add -1.3442 to $A$ and 0.7312 to B. For other geographical regions, except North America: add -0.2817 to A and 0.4966 to $B$. 
Supplemental Figure 1: Distribution in the combined study populations of (a) 10-year absolute risks for MACE; (b) 10-year absolute risk reductions for MACE with added rivaroxaban; (c) 10-year absolute risks of major bleeding; (d) 10-year absolute risk reductions for major bleeding with added rivaroxaban.
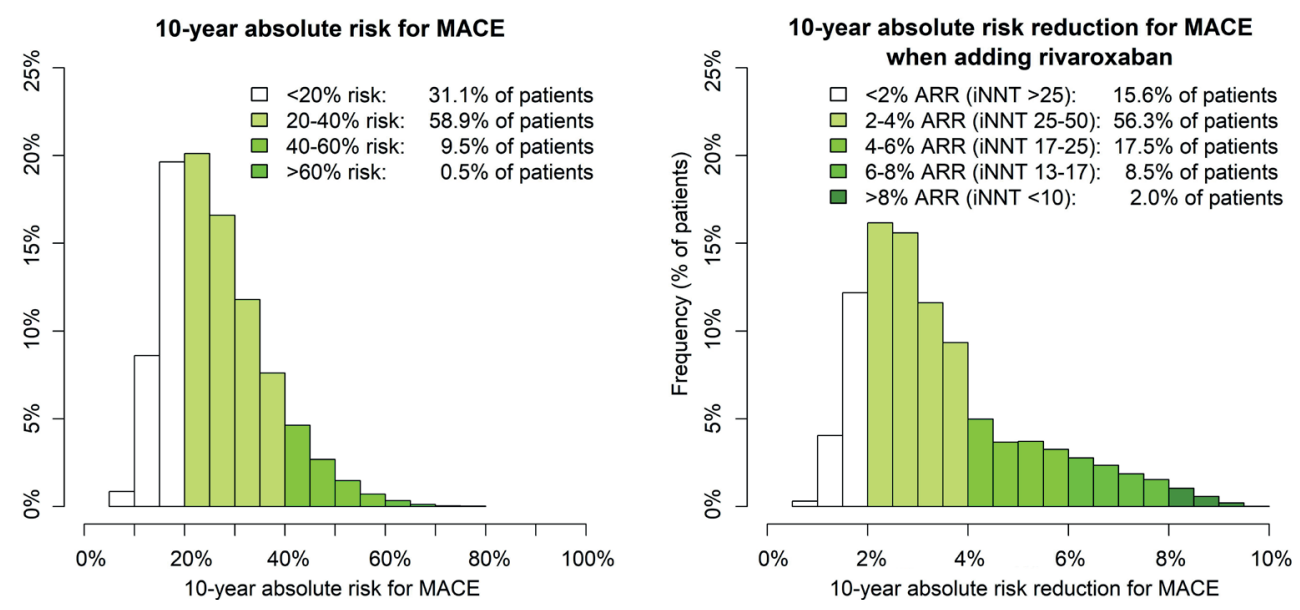

\section{0-year absolute risk for major bleeding}
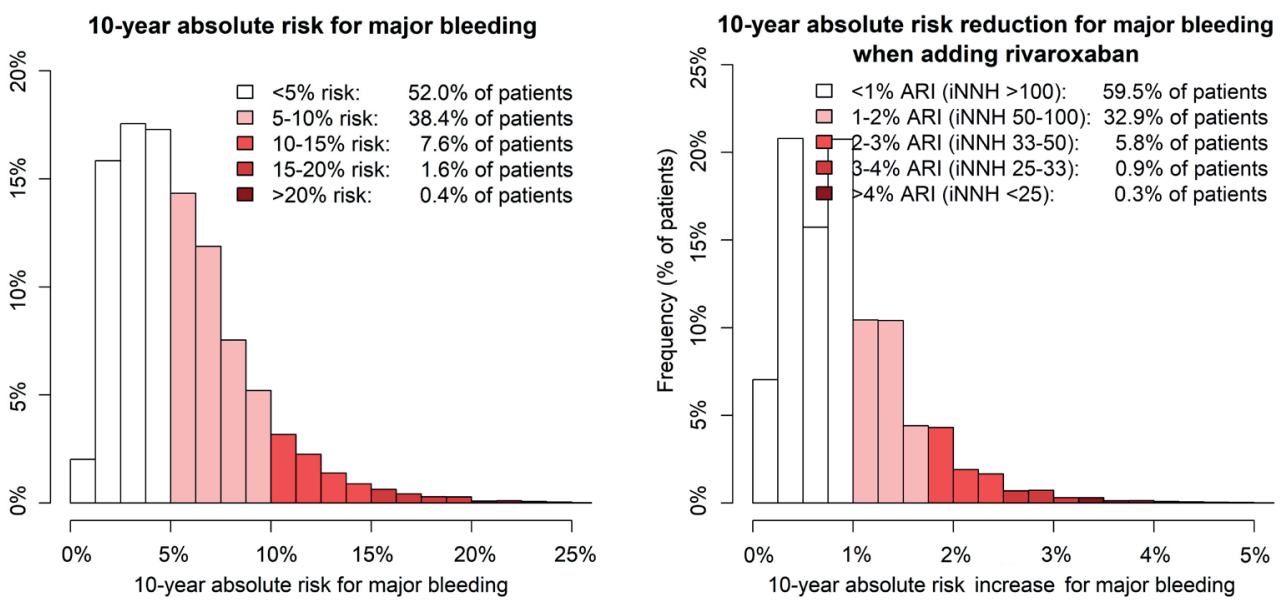


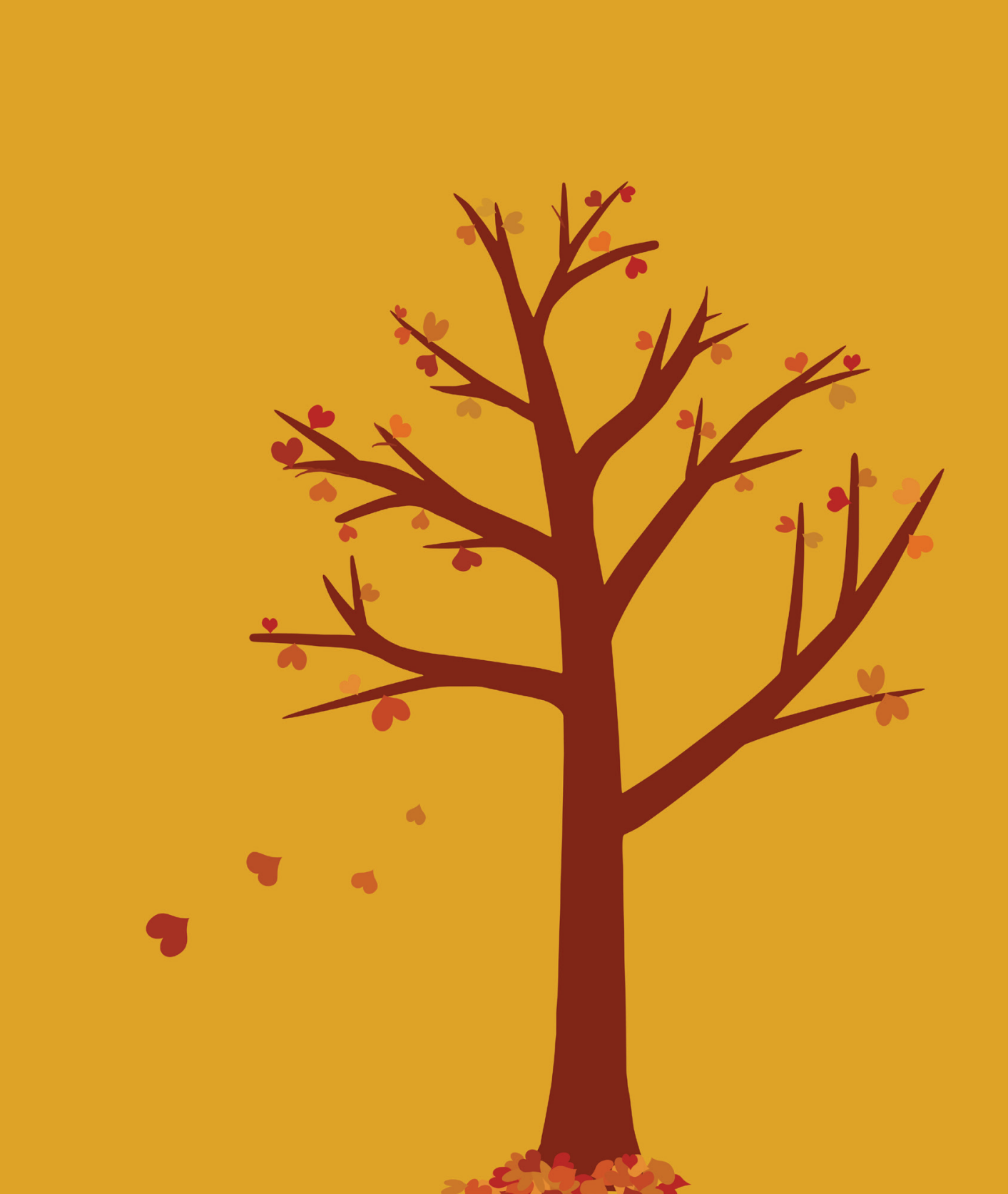


Heterogeneity of treatment effects from an intensive lifestyle weight loss intervention on cardiovascular events in patients with type 2 diabetes: data from the Look AHEAD trial.

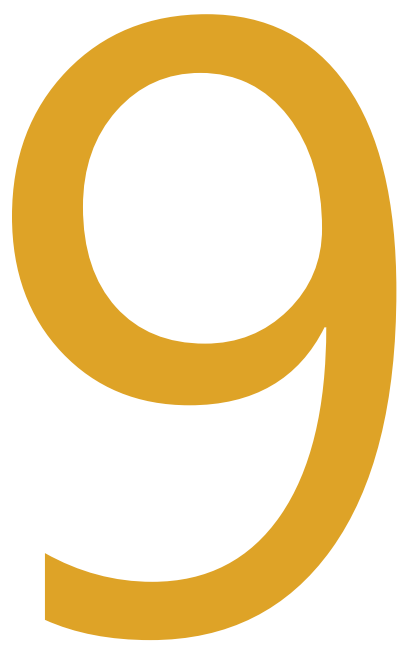

Tamar I. de Vries Jannick A.N. Dorresteijn Yolanda van der Graaf Frank L.J. Visseren Jan Westerink 


\section{ABSTRACT}

\section{Objective}

To explore the presence of heterogeneity of treatment effect (HTE) of an intensive lifestyle intervention (ILI) on the occurrence of major cardiovascular events (MACE) in overweight or obese patients with type 2 diabetes mellitus; and to identify patient characteristics associated with individual treatment effect.

\section{Methods}

In 4,901 participants from the Action for Health in Diabetes (Look AHEAD) trial, a penalized Cox regression model to predict treatment effect of ILI for the risk of MACE was derived including all possible treatment-by-covariate interaction terms. The ability of the model to predict HTE was confirmed by calculating hazard ratios (HR) and absolute risk change in quartiles of predicted treatment effect, and baseline patient characteristics were compared between quartiles.

\section{Results}

In quartile 1 of predicted treatment effect, with the highest predicted risk reduction, there was a significant treatment benefit of ILI (HR 0.64; 95\% confidence interval [95\% CI] 0.49 0.83), while there was no effect from treatment in quartiles 2 and 3 (HR 0.81, 95\% Cl 0.58 1.14 , and $1.13,95 \% \mathrm{Cl} 0.80-1.60$, respectively), and a detrimental effect in quartile 4 (HR 1.37, 95\% Cl 1.09-1.73). Several patient characteristics in demographics, medical history, physical examination, and laboratory values were associated with the level of treatment effect.

\section{Conclusion}

This post-hoc analysis of the Look AHEAD trial showed that an intensive lifestyle intervention aimed at weight loss may reduce cardiovascular events in selected patients, but may have a detrimental treatment effect in others. 


\section{Background}

In patients with type 2 diabetes, it has been demonstrated that weight loss has beneficial effects on metabolic control and cardiovascular risk factors, ${ }^{1,2}$ and bariatric surgery may decrease the risk of cardiovascular events in obese patients with type 2 diabetes. ${ }^{3}$ However, it has not been demonstrated that weight loss through lifestyle intervention also has a positive effect on cardiovascular outcomes. The Action for Health in Diabetes trial (Look AHEAD) randomized overweight and obese patients with type 2 diabetes to an intensive lifestyle intervention program that promoted weight loss through decreased caloric intake and increased physical activity, or to a control group with regular diabetes support and education. ${ }^{4}$ It was ended prematurely on basis of a futility analysis after a median follow-up of 9.6 years. Although the intervention lead to greater weight loss and greater reductions in glycated haemoglobin ( $\mathrm{HbA1c}$ ) and cardiovascular risk factors, there was no reduced risk of cardiovascular events or mortality in the intervention group compared to the control group. ${ }^{1}$

Subgroup analyses in the Look AHEAD trial have shown that in several subgroups, the lifestyle program led to an important reduction of cardiovascular risk factors. A posthoc analysis has shown that individuals who lost more than $10 \%$ of their body weight, had a significantly lower risk of major cardiovascular outcomes (MACE). ${ }^{5}$ These findings suggest that lifestyle modification may have a beneficial effect in reducing cardiovascular outcomes in individual patients or patient subgroups, but no subgroups based on single baseline characteristics were identified with a significant treatment effect on cardiovascular outcomes. However, treatment decisions based on group level are suboptimal, as they are based on single patient characteristics while it may be possible that a combination of patient characteristics influences the treatment effect from an intervention. Furthermore, most trials do not have enough power to study treatment effects in subgroups. ${ }^{6}$ The question therefore remains whether intensive lifestyle interventions can be beneficial in reducing cardiovascular events in individual patients, when no treatment effect has been found on average.

The aim of this post-hoc analysis of the Look AHEAD trial is to explore the possible presence of heterogeneity of treatment effect (HTE) of an intensive lifestyle intervention on the occurrence of major cardiovascular events (MACE) in overweight or obese patients with type 2 diabetes mellitus, and to identify patient characteristics associated with heterogeneity in treatment effect. 


\section{Methods}

\section{Data acquisition and study population}

We submitted a research proposal for the current study at the National Institute of Diabetes and Digestive and Kidney Diseases Central Repositories. The proposal was reviewed by an independent review panel, and access to the anonymized individual patient data from the Look AHEAD was provided.

The Look AHEAD trial (trial registration NCT00017953) included 5,145 overweight and obese patients with type 2 diabetes mellitus, of which 4,901 are available in the public access datasets, as those participants participating from Native American sites are excluded, per consent limitations. The design and methods of Look AHEAD have been reported elsewhere. ${ }^{4}$ In short, participants were recruited from 16 clinical centers in the United States, who were then randomized to either diabetes support and education (control) or intensive lifestyle intervention. Trial enrolment began in 2001, with follow-up continuing through 2012. The Look Ahead trial complied with the Declaration of Helsinki, ethical approval was obtained from local Institutional Review Boards, and all study participants provided written informed consent.

\section{Study end points}

As defined in the trial, the primary endpoint was the first occurrence of four-point MACE, a composite endpoint of nonfatal myocardial infarction or stroke, hospitalization for angina, and death from cardiovascular causes.

\section{Data analysis}

Covariate data were missing in $<1 \%$ of study participants and were imputed using single imputation using predictive mean matching, based on other patient characteristics and outcomes. All analyses were conducted with R statistical software V.3.4.1 (www.r-project. org), using add-on packages Hmisc, survival, and penalized. ${ }^{7}$ For all analyses, a p-value $<0.05$ was considered statistically significant, unless noted otherwise.

Model development and internal validation

To model treatment effect, first, a Cox proportional hazard functions for the prediction of MACE was derived in the Look AHEAD trial population. The model contained the following pre-specified predictors: age, sex, current smoking, history of CVD, use of insulin, the duration of diabetes, systolic blood pressure (SBP; in $\mathrm{mmHg}$ ), non-high density lipoprotein (non-HDL) cholesterol (in mmol/L), HbA1c (in $\mathrm{mmol} / \mathrm{mol}$ ), estimated glomerular filtration rate (eGFR, measured according to the CKD-EPI formula; in $\mathrm{ml} / \mathrm{min} / 1.73 \mathrm{~m}^{2}$ ), body mass index $\left(\mathrm{BMI}\right.$, in $\left.\mathrm{kg} / \mathrm{m}^{2}\right)$, and the presence of micro- or macro-albuminuria. The choice for these predictors was based on a recently developed life-time risk model in patients with 
type 2 diabetes. ${ }^{8}$ To model treatment effect directly, treatment-by-covariate interaction terms for all included predictors were added to the model.6,9 To avoid chance findings and overfitting, no statistical selection was applied. Thus, all pre-selected predictors and interactions were included in the model. Continuous predictors were truncated at the $1^{\text {st }}$ and $99^{\text {th }}$ percentile to limit the effects of outliers. To improve the robustness of the model, transformation was applied for continuous variables when this improved model fit based on Akaike's Information Criterion (AIC). ${ }^{10}$ The proportional hazards assumption was checked by visually assessing the scaled Schoenfeld residuals. The final model coefficients were estimated using penalized estimation methods using an L2 quadratic (i.e. "ridge") penalty to further prevent overfitting. ${ }^{6,7}$

The predictive value of the model was assessed based on calibration plots of predicted versus observed 10-year risks of MACE and the c-statistic as a measure for discrimination.

Individual treatment effect estimations

Subsequently, the newly derived prediction model was used to estimate the 10-year risk of MACE for each study participant using two scenarios: as if they had been treated (a) with diabetes support and education or (b) with intensive lifestyle intervention. The treatment effect was defined as the 10-year risk with diabetes support and education minus the 10year risk with the intensive lifestyle intervention.

Identifying heterogeneity of treatment effect

Next, to assess HTE for intensive lifestyle intervention, the study population was divided into quartiles based on predicted treatment effect. The hazard ratios (HR) and associated 95\% confidence intervals $(95 \% \mathrm{Cl}$ ) for the effect of treatment per quartiles were obtained with Cox proportional hazard models corrected for the prognostic variables used in the risk models to ensure that no confounding had been induced by the division of the study population in quartiles.

Baseline characteristics were described for the four quartiles to identify characteristics that are associated with a high versus low predicted benefit from an intensive lifestyle intervention for the risk of MACE. The differences between these patient characteristics over quartiles of baseline risk were compared using Kruskal-Wallis tests for continuous variables and Chi-square tests for categorical variables, with Bonferroni correction for multiple testing. Furthermore, in order to generate hypotheses about the causes of possible HTE, we graphically displayed changes in body weight, waist circumference, $\mathrm{HbA1c}$, SBP, and low-density lipoprotein (LDL) cholesterol during the study period stratified for quartiles 1 and 4 , and for treatment allocation. 


\section{Results}

Of the patients included in the trial, 2448 (50\%) were allocated to the intensive lifestyle intervention arm. Patients included in the trial were on average 59 years old, 41\% was male, and $14 \%$ had a history of cardiovascular disease. The mean BMI was $36 \mathrm{~kg} / \mathrm{m}^{2}$. The median duration of type 2 diabetes before inclusion in the trial was 5 years. Detailed baseline characteristics of the study population are shown in Supplemental Table 1 for the entire study population, and in Table 1 stratified for quartiles of estimated treatment effect. The median follow-up in the trial was 9.4 years (interquartile range 8.5-10.2), during which MACE occurred 799 times.

Table 1. Patient characteristics in quartiles of predicted treatment effect

\begin{tabular}{llllll}
\hline & $\begin{array}{l}\text { Quartile 1 } \\
(n=1,226)\end{array}$ & $\begin{array}{l}\text { Quartile 2 } \\
(n=1,225)\end{array}$ & $\begin{array}{l}\text { Quartile 3 } \\
(n=1,225)\end{array}$ & $\begin{array}{l}\text { Quartile 4 } \\
(n=1,225)\end{array}$ & p-value \\
\cline { 2 - 5 } Allocated to intensive lifestyle intervention & $600(49 \%)$ & $600(49 \%)$ & $651(53 \%)$ & $597(49 \%)$ &
\end{tabular}

\section{Demographics}

Age (years)

Male sex

Current smoking

Ethnicity

White / Caucasian

Black / African-American

Hispanic

Other

$\begin{array}{lllll}63 \pm 6 & 59 \pm 6 & 56 \pm 6 & 58 \pm 7 & <.0001^{*} \\ 692(56 \%) & 441(36 \%) & 313(26 \%) & 584(48 \%) & <.0001^{*} \\ 32(3 \%) & 29(2 \%) & 45(4 \%) & 102(8 \%) & <.0001^{*} \\ & & & & <.0001^{*} \\ 882(72 \%) & 843(69 \%) & 762(62 \%) & 760(62 \%) & \mathrm{N} / \mathrm{A} \\ 146(12 \%) & 183(15 \%) & 242(20 \%) & 233(19 \%) & \mathrm{N} / \mathrm{A} \\ 141(12 \%) & 153(12 \%) & 186(15 \%) & 196(16 \%) & \mathrm{N} / \mathrm{A} \\ 57(5 \%) & 46(4 \%) & 35(3 \%) & 36(3 \%) & \mathrm{N} / \mathrm{A}\end{array}$

\section{Medical history and medication use}

History of cardiovascular disease

Duration of diabetes (years), median

Use of insulin

Use of statin

Use of BP lowering medication

SF-36 general health score

$\begin{array}{lllll}124(10 \%) & 48(4 \%) & 63(5 \%) & 455(37 \%) & <.0001^{*} \\ 7(3-13) & 4(2-8) & 4(2-7) & 5(3-10) & <.0001^{*} \\ 205(17 \%) & 144(12 \%) & 164(13 \%) & 319(26 \%) & <.0001^{*} \\ 550(45 \%) & 539(44 \%) & 545(44 \%) & 657(54 \%) & <.0001^{*} \\ 959(78 \%) & 879(72 \%) & 814(66 \%) & 896(73 \%) & <.0001^{*} \\ 48 \pm 9 & 48 \pm 9 & 47 \pm 9 & 46 \pm 9 & <.0001^{*}\end{array}$

\section{Physical examination}

\begin{tabular}{llllll} 
Weight $(\mathrm{kg})$ & $101 \pm 19$ & $102 \pm 20$ & $99 \pm 19$ & $102 \pm 19$ & $<.0001^{*}$ \\
Body mass index $\left(\mathrm{kg} / \mathrm{m}^{2}\right)$ & $35 \pm 6$ & $36 \pm 6$ & $36 \pm 6$ & $36 \pm 6$ & $0.0001^{\star}$ \\
Waist circumference $(\mathrm{cm})$ & $114 \pm 13$ & $114 \pm 14$ & $112 \pm 14$ & $115 \pm 14$ & $<.0001^{\star}$ \\
\hline
\end{tabular}


Table 1. (continued)

\begin{tabular}{llllll}
\hline & Quartile 1 & Quartile 2 & Quartile 3 & Quartile 4 & \\
\cline { 2 - 5 } $\begin{array}{l}\text { Systolic blood pressure (mmHg) } \\
\text { Diastolic blood pressure (mmHg) }\end{array}$ & $139 \pm 17$ & $130 \pm 15$ & $123 \pm 15$ & $124 \pm 16$ & $<.0001^{*}$ \\
& $73 \pm 10$ & $70 \pm 9$ & $69 \pm 9$ & $69 \pm 10$ & $<.0001^{*}$ \\
Laboratory tests & & & & & \\
Glycated haemoglobin (\%) & $6.7 \pm 0.8$ & $6.7 \pm 0.8$ & $7.2 \pm 0.7$ & $8.5 \pm 1.3$ & $<.0001^{*}$ \\
Glycated haemoglobin (mmol/mol) & $50 \pm 9$ & $50 \pm 9$ & $55 \pm 8$ & $69 \pm 14$ & $<.0001^{*}$ \\
HDL cholesterol (mmol/L) & $1.1 \pm 0.3$ & $1.1 \pm 0.3$ & $1.2 \pm 0.3$ & $1.1 \pm 0.3$ & $<.0001^{*}$ \\
LDL cholesterol (mmol/L) & $3.0 \pm 0.9$ & $2.9 \pm 0.8$ & $2.8 \pm 0.7$ & $2.9 \pm 0.9$ & $<.0001^{*}$ \\
Triglycerides (mmol/L) & $2.1 \pm 1.3$ & $2.0 \pm 1.2$ & $1.9 \pm 1.1$ & $2.2 \pm 1.6$ & $<.0001^{*}$ \\
Creatinine ( $\mu$ mol/L), median (IQR) & $88(71-97)$ & $80(63-88)$ & $71(62-88)$ & $80(71-97)$ & $<.0001^{*}$ \\
Presence of albuminuria & & & & & $<.0001^{*}$ \\
Micro-albuminuria & $412(34 \%)$ & $124(10 \%)$ & $46(4 \%)$ & $82(7 \%)$ & $\mathrm{N} / \mathrm{A}$ \\
Macro-albuminuria & $13(1 \%)$ & $13(1 \%)$ & $20(2 \%)$ & $89(7 \%)$ & $\mathrm{N} / \mathrm{A}$
\end{tabular}

\section{Socio-economic status}

Highest level of education

High school or less

$\begin{array}{lllll}221(18 \%) & 219(18 \%) & 233(19 \%) & 257(21 \%) & \text { N/A } \\ 455(37 \%) & 477(39 \%) & 496(40 \%) & 468(38 \%) & \text { N/A }\end{array}$

College graduate

$549(45 \%) \quad 529(43 \%) \quad 496(40 \%) \quad 499(41 \%) \quad N / A$

Income in the last 12 months

$<\$ 10,000$

$90(9 \%) \quad 96(10 \%)$

$127(12 \%)$

$150(14 \%)$

$<.0001^{\star}$

$\$ 10,000-\$ 100,000$

$148(15 \%) \quad 168(17 \%)$

$220(21 \%)$

$204(20 \%)$

N/A

$>\$ 100,000$

$745(76 \%) \quad 743(74 \%)$

$687(66 \%)$

$688(66 \%)$

N/A

$I Q R=$ interquartile range; $N / A=$ not applicable; $B P=$ blood pressure

All values are presented as either $n(\%)$, mean \pm standard deviation unless noted otherwise

* $=$ statistically significant at the Bonferroni-corrected alpha

\section{Individual risk and treatment effect predictions}

Supplemental Table 2 shows the formula for the estimation of the risk of MACE that was used for the predictions. Supplemental Figure 1 shows good agreement between the predicted and observed risk of MACE in the study population (internal validation); the c-statistic for discrimination was 0.73 (95\% Cl: 0.71-0.75).

The median estimated baseline 10 year risk for MACE when treated with the control treatment was 15\% (range 0.3-96\%; Figure 1). The median estimated absolute treatment effect on 10 year risk for MACE with lifestyle intervention was $-1.3 \%$ and varied substantially, ranging from $-39 \%$ to $+43 \%$ (Figure 1 ). 
Figure 1. Histograms showing the distribution of (left) the 10-year predicted baseline risk of the primary outcome four-point major cardiovascular events (MACE) with Diabetes Support and Education; (right) the 10-year absolute treatment effect on the risk of MACE with an intensive lifestyle intervention.
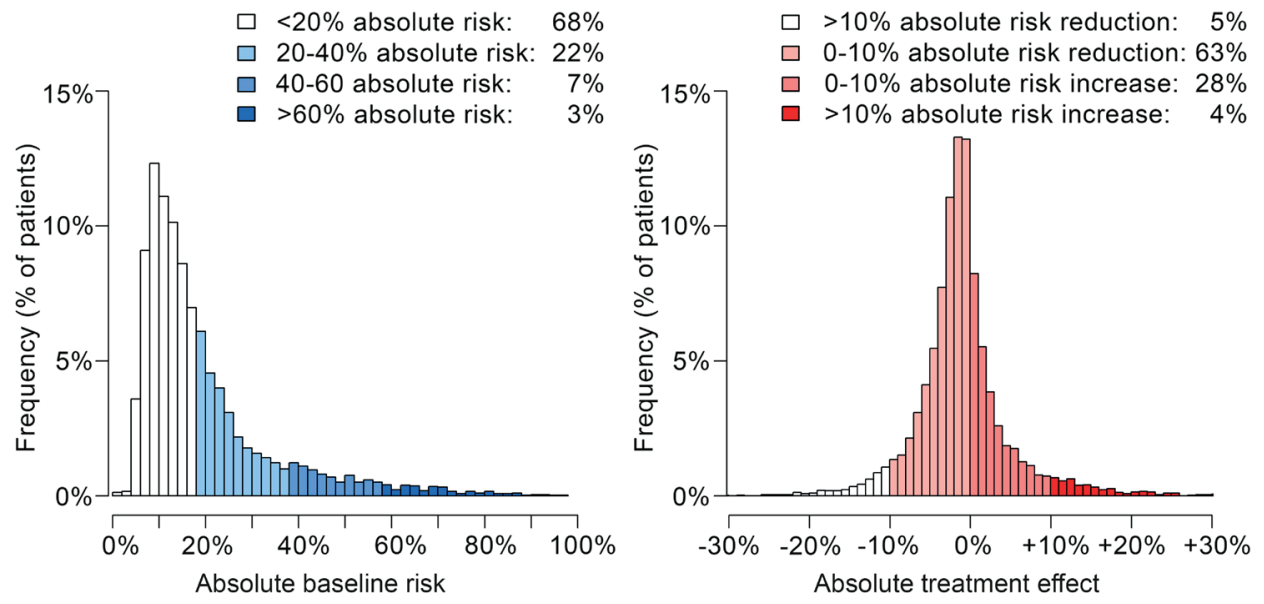

\section{Heterogeneity of treatment effects}

Figure 2 shows the event rates in both treatment arms, the associated hazard ratios and the median absolute treatment effect stratified for quartiles of estimated treatment effect. Cox proportional hazard models adjusted for all prognostic factors included in the risk model showed an observed benefit of intervention versus control in quartile 1 (HR 0.64; $95 \% \mathrm{Cl} 0.49-0.83)$, no statistically significant treatment effect from treatment in quartiles 2 and 3 (HR 0.81, 95\% Cl 0.58-1.14, and 1.13, 95\% Cl 0.80-1.60, respectively), and a detrimental effect of intervention in quartile 4 (HR 1.37, 95\% Cl 1.09-1.73). Table 1 shows the baseline patient characteristics stratified for the quartiles of predicted treatment effect. Figure 3 shows the percentage change from baseline in body weight, waist circumference, HbA1c, SBP, and LDL cholesterol during 10 years of follow-up in quartiles 1 and 4 stratified for trial allocation. 
Figure 2. Risk of major cardiovascular events (MACE) and treatment effects from intensive lifestyle intervention (ILI) versus diabetes support and education (DSE) arms in the Look AHEAD trial, stratified in quartiles of predicted therapy benefit from ILI on the risk of MACE. (A) event rates of MACE in the DSE versus ILI groups; (B) hazard ratios (with $95 \%$ confidence interval) of ILI versus DSE; (C) median (with interquartile range) absolute treatment effect of ILI versus DSE.
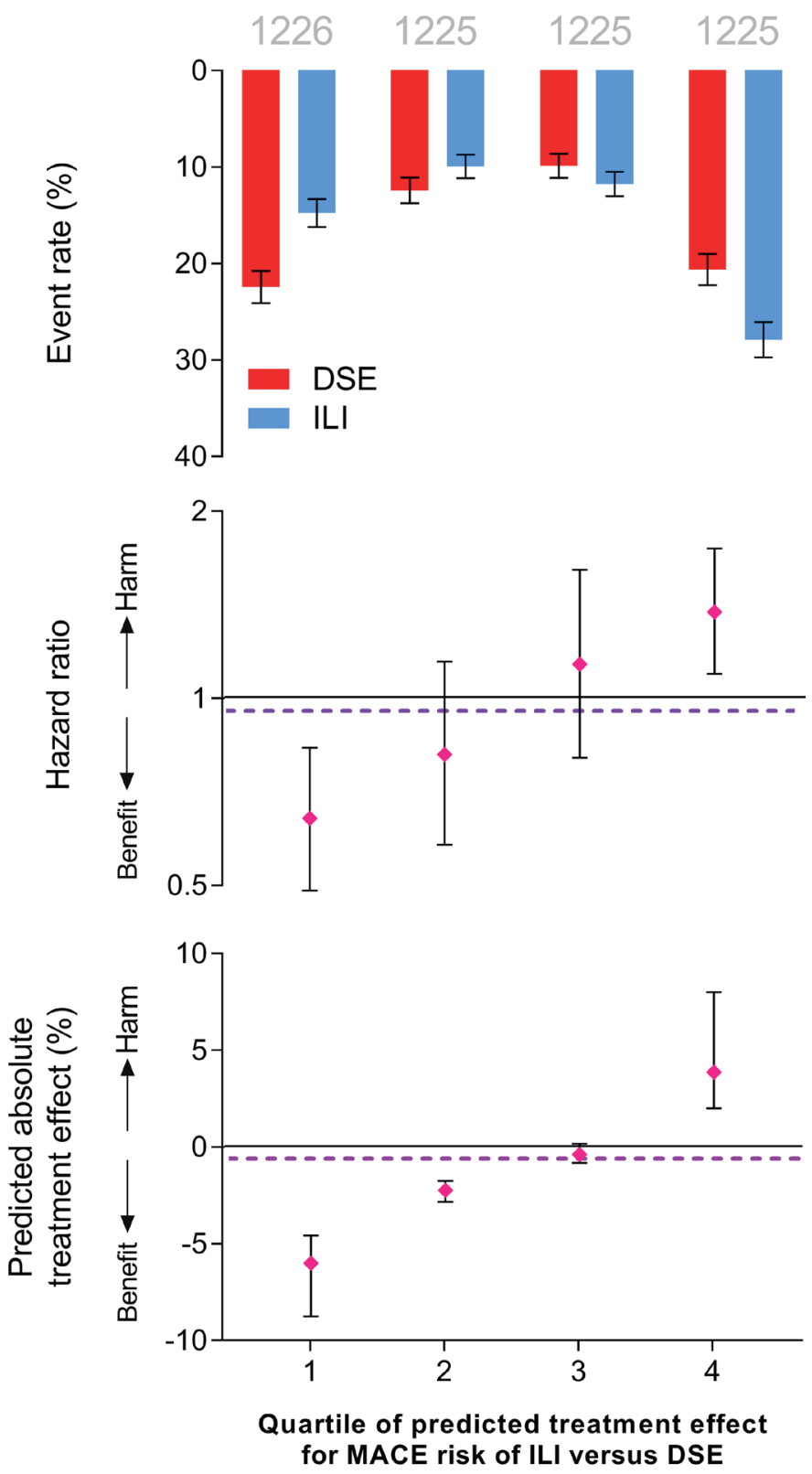
Figure 3. Changes from baseline in weight, waist circumference, glycated haemoglobin, systolic blood pressure, and low-density lipoprotein (LDL) cholesterol during 4 years of follow-up in patients from the Look AHEAD trial, shown for the quartiles with respectively the highest or lowest predicted benefit from intervention, and stratified for the treatment allocation in the trial (ILI = intensive lifestyle intervention; DSE = diabetes support and education)

\section{(A) Weight}

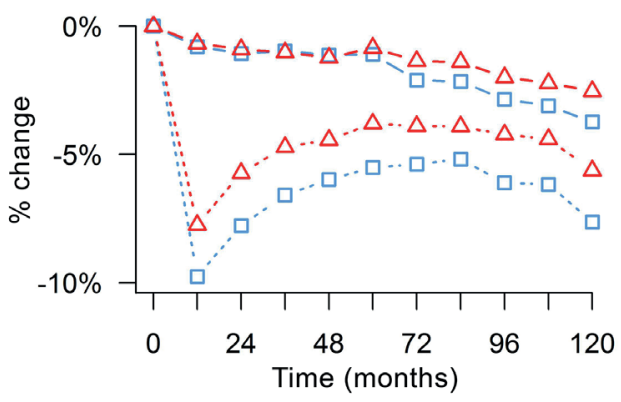

(B) Waist circumference

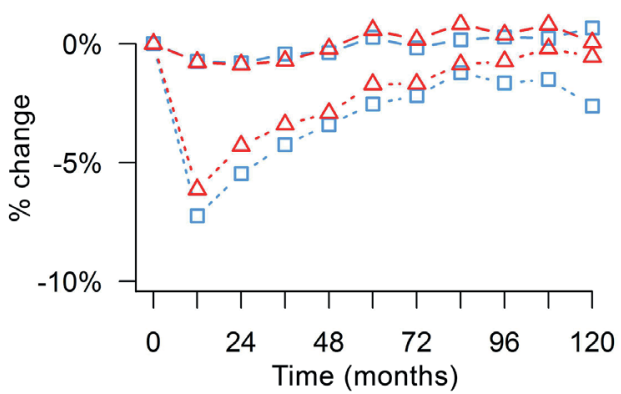

(C) Glycated hemoglobin

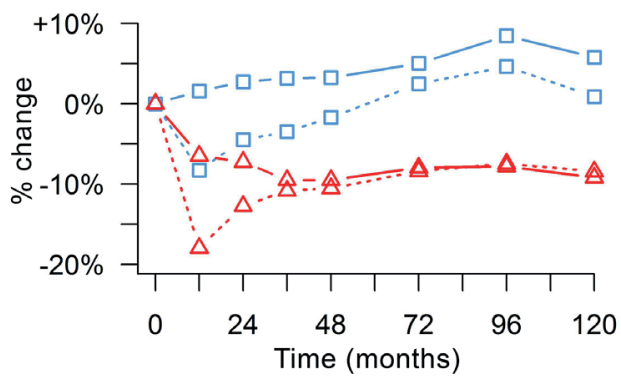

(D) Systolic blood pressure

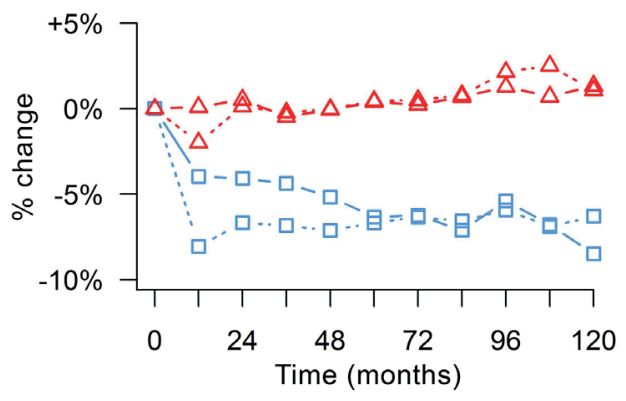

(E) LDL cholesterol

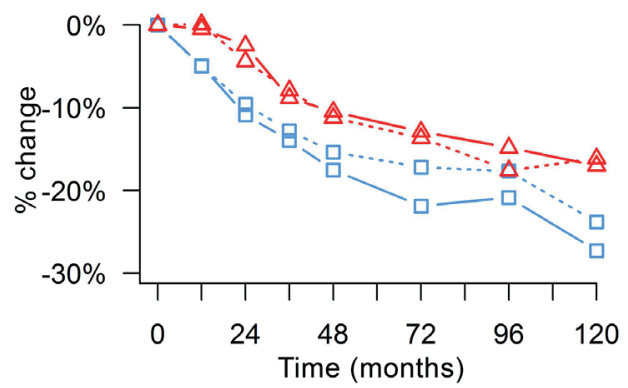

Legend

--E-- Quartile 1 intervention (ILI) arm

- Quartile 1 control (DSE) arm

- $\Delta$ - Quartile 4 intervention (ILI) arm

$\triangle$ Quartile 4 control (DSE) arm 


\section{Discussion}

This exploratory analysis of the Look AHEAD trial demonstrated heterogeneity in treatment effects from an intensive lifestyle intervention on the occurrence of MACE in overweight and obese patients with type 2 diabetes. Furthermore, patient characteristics were identified that are associated with possible HTE, including patient demographics, medical history, measures of socio-economic status, and laboratory values.

Currently, most international guidelines for type 2 diabetes include recommendations of lifestyle interventions. ${ }^{11-13}$ The European Association of Preventive Cardiology recently published a position paper stressing the importance of exercise training in patients with type 2 diabetes and cardiovascular disease, based on the potential of exercise to improve cardiovascular and metabolic functions, despite the lack of evidence of a positive effect on cardiovascular risk. ${ }^{14}$ In the current study, it is demonstrated that there is a subgroup of patients in the Look AHEAD trial who did benefit from a lifestyle intervention aimed at weight loss in terms of a reduction in the risk of MACE, confirming the importance of lifestyle interventions in at least part of the population with type 2 diabetes.

Based on the results from the current study, however, there may also be a group of patients in whom an intensive lifestyle intervention has a detrimental effect on CVD-free survival. It is however important to realize that the intervention in the Look AHEAD trial is a specific and intensive lifestyle intervention and these results may be different in other lifestyle programs. The Look AHEAD intervention aims at a low caloric intake (1200-1800 kcal per day) and an increased physical activity (at least 175 minutes of moderate-intensity physical activity per week). It is unclear how different weight loss lifestyle interventions would influence the findings of the current study. Furthermore, the lifestyle intervention in Look AHEAD has been found to improve e.g. quality of life, ${ }^{15}$ mobility, ${ }^{16}$ sleep apnoea, ${ }^{17}$ sexual dysfunction, ${ }^{18}$ and depression, ${ }^{19}$ and improved glycaemic control will also benefit the risk of microvascular complications. ${ }^{12}$

Nonetheless, based on the results from the current study, it may be wise to be cautious with regards to very intensive lifestyle interventions such as the intervention from the Look AHEAD trial in certain patient categories. Future research should be aimed at investigating which types of (intensive) lifestyle interventions are effective and safe to use in these subgroups of overweight and obese patients with type 2 diabetes.

The current study identifies several patient characteristics that differ between quartiles of predicted treatment effect (as shown in Table 1), while in (pre-specified) simple subgroup analyses no baseline characteristics were identified that modified the treatment effect of the intensive lifestyle intervention. In a pre-specified subgroup analysis, a nonsignificantly higher event rate for the primary outcome was found in the intervention 
arm compared to the control arm in the subgroup with patients with a history of CVD, versus a non-significantly lower event rate in patients without CVD, which is in line with the findings in the current study. A post-hoc, machine learning based analysis identified higher $\mathrm{HbA1c}$ levels as a characteristic associated with treatment benefit, ${ }^{20}$ which is in contrast with the findings in the current study. This difference may be explained by the multivariable approach of the current study, compared with the subgroup-based approach of the machine learning-based study. Subgroup analyses are univariable analyses, whereas HTE likely cannot be explained by single patient characteristics only. ${ }^{6}$ Importantly, while certain characteristics may have been found to be associated with the treatment effect of intensive lifestyle intervention in Look AHEAD, this does not necessarily imply a causal relation between this characteristic and the modified treatment response. It may prove to be difficult to disentangle the relation between these risk factors and HTE. For example, while the proportion of current smokers is higher in quartile 4 compared to the other quartiles, smoking status is also associated with having a history of cardiovascular disease, and with socio-economic status. Using the current methodology, it is not possible to prove which of these risk factors - if any - are independent and causal treatment effect modifiers.

However, although the methodology of the current study is not suitable for investigating causal relationships, it can be used to generate hypotheses about possible mechanisms underlying the HTE found in the study.

In the Action to Control Cardiovascular Risk in Diabetes (ACCORD) trial, which randomized patients with a history of or a high risk for cardiovascular disease to either strict or standard glycaemic control, an increased risk of mortality was seen in the intensive glycaemic control arm. ${ }^{21}$ This increase was most prominent in patients with a high baseline HbA1c (>8.5\% or $69 \mathrm{mmol} / \mathrm{mol}$ ). ${ }^{22}$ Subsequent analyses found that this was partly, though not fully, explained by the increased risk of hypoglycaemia. In ACCORD, the risk of hypoglycaemia was higher in African-Americans, those with lower education levels, those with higher baseline HbA1c levels, those with signs of nephropathy, and users of insulins, ${ }^{23,24}$ characteristics that are also associated with a detrimental effect of treatment in the current study. It is possible that the risk of hypoglycaemia may partly explain the increased risk in part of the study population in Look AHEAD. In line with the findings in the ACCORD trial, the current study found that in the quartile with the largest risk increase, the mean baseline HbA1c levels were highest, and the decline in HbA1c during the study period was markedly steeper than in those patients with a predicted risk reduction (those who would benefit from the intensive lifestyle intervention), as shown in Figure 2. In ACCORD, however, not all of the increased risk of mortality could be explained by hypoglycaemia, as is the case in the present study, and it is still uncertain what other underlying mechanisms are present. 
The current study has several strengths. First of all, a multivariable risk prediction-based approach was used to deal with several limitations from subgroup analyses. ${ }^{6}$ Secondly, using a risk-based approach to define quantiles of treatment effect, randomization remains intact within these quantiles. To further ensure that no confounding has been induced by chance during stratification, the hazard ratios of intervention per quartile were corrected for prognostic factors.

Several limitations should also be acknowledged. Firstly, it is important to note that these analyses are exploratory and not pre-specified in the trial. Therefore, the conclusions should be interpreted with caution. Additionally, differences of baseline characteristics between quartiles of treatment effect were at least partially created by the choice of predictors used in the prediction model. Thirdly, the results from this study cannot be used to make causal inferences, but are merely hypotheses-generating. Fourthly, we did not have data available to analyse the presence of hypoglycaemia, variability in glucose control, or haemoglobin glycation index as potential mechanisms underlying the potential treatment effects. Finally, as the treatment effect-based model was derived within the Look AHEAD data, it is possible that there is overfitting of this model to the data, which gives the risk of false discoveries. ${ }^{6}$ However, to limit this risk, we used pre-specified predictors and estimated the final model using penalized regression.

In conclusion, this exploratory study of the Look AHEAD trial shows heterogeneity in the treatment effect from an intensive lifestyle intervention aimed at weight loss on the occurrence of MACE in overweight and obese patients with type 2 diabetes. Using an approach based on treatment effect modelling, it is possible to identify subgroups of patients with a possible beneficial or potentially even detrimental treatment effect of the intensive lifestyle intervention used in the Look AHEAD trial on cardiovascular outcomes. Patient characteristics associated with a potential treatment benefit are, among other, no history of cardiovascular disease, good control of type 2 diabetes, no use of insulin, higher socio-economic status, and the absence of macro-albuminuria. Future research into intensive lifestyle weight loss interventions for cardiovascular disease risk reduction should on the one hand be specifically aimed at subgroups of patients with a higher likelihood of treatment benefit, and on the other hand at finding safe lifestyle interventions for subgroups of patients with a potential treatment harm. 


\section{References}

1. The Look AHEAD Research Group, Wing RR, Bolin P, Brancati FL, Bray GA, Clark JM, et al. Cardiovascular Effects of Intensive Lifestyle Intervention in Type 2 Diabetes. N Engl J Med. 2013;369(2):145-54.

2. Schauer PR, Bhatt DL, Kirwan JP, Wolski K, Aminian A, Brethauer SA, et al. Bariatric Surgery versus Intensive Medical Therapy for Diabetes - 5-Year Outcomes. N Engl J Med. 2017;376(7):641-51.

3. Romeo S, Maglio C, Burza MA, Pirazzi C, Sjoholm K, Jacobson P, et al. Cardiovascular events after bariatric surgery in obese subjects with type 2 diabetes. Diabetes Care. 2012;35(12):2613-7.

4. Ryan DH, Espeland MA, Foster GD, Haffner SM, Hubbard VS, Johnson KC, et al. Look AHEAD (Action for Health in Diabetes): design and methods for a clinical trial of weight loss for the prevention of cardiovascular disease in type 2 diabetes. Control Clin Trials. 2003;24(5):610-28.

5. Gregg E, Jakicic J, Blackburn G, Bloomquist P, Bray G, Clark J, et al. Association of the magnitude of weight loss and changes in physical fitness with long-term cardiovascular disease outcomes in overweight or obese people with type 2 diabetes: a post-hoc analysis of the Look AHEAD randomised clinical trial. Lancet Diabetes Endocrinol. 2016;4(11):913-21.

6. Kent DM, Steyerberg E, van Klaveren D. Personalized evidence based medicine: predictive approaches to heterogeneous treatment effects. BMJ. 2018;363:k4245.

7. Goeman JJ. L1 penalized estimation in the Cox proportional hazards model. Biom J. 2010;52(1):7084.

8. Berkelmans GF, Gudbjörnsdottir S, Visseren FL, Wild SH, Franzen S, Chalmers J, et al. Prediction of individual life-years gained without cardiovascular events from lipid, blood pressure, glucose, and aspirin treatment based on data of more than 500000 patients with Type 2 diabetes mellitus. Eur Heart J. 2019; [Epub ahead of print].

9. Farooq V, van Klaveren D, Steyerberg EW, Meliga E, Vergouwe Y, Chieffo A, et al. Anatomical and clinical characteristics to guide decision making between coronary artery bypass surgery and percutaneous coronary intervention for individual patients: development and validation of SYNTAX score II. Lancet. 2013;381(9867):639-50.

10. Steyerberg EW. Clinical prediction models: a practical approach to development, validation and updating. New York, USA: Springer; 2009.

11. Davies MJ, D'Alessio DA, Fradkin J, Kernan WN, Mathieu C, Mingrone G, et al. Management of hyperglycaemia in type 2 diabetes, 2018. A consensus report by the American Diabetes Association (ADA) and the European Association for the Study of Diabetes (EASD). Diabetologia. 2018;61(12):2461-98.

12. Piepoli MF, Hoes AW, Agewall S, Albus C, Brotons C, Catapano AL, et al. 2016 European guidelines on cardiovascular disease prevention in clinical practice: the Sixth Joint Task Force of the European Society of Cardiology and Other Societies on Cardiovascular Disease Prevention in Clinical Practice. Eur Heart J. 2016;37(29):2315-81.

13. Lifestyle Management: Standards of Medical Care in Diabetes-2018. Diabetes Care. 2018 
Jan;41(Suppl 1):S38-50.

14. Kemps $H$, Krankel $N$, Dorr $M$, Moholdt $T$, Wilhelm M, Paneni $F$, et al. Exercise training for patients with type 2 diabetes and cardiovascular disease: What to pursue and how to do it. A Position Paper of the European Association of Preventive Cardiology (EAPC). Eur J Prev Cardiol. 2019;2047487318820420.

15. Williamson DA, Rejeski J, Lang W, Van Dorsten B, Fabricatore AN, Toledo K. Impact of a weight management program on health-related quality of life in overweight adults with type 2 diabetes. Arch Intern Med. 2009;169(2):163-71.

16. Rejeski WJ, Ip EH, Bertoni AG, Bray GA, Evans G, Gregg EW, et al. Lifestyle Change and Mobility in Obese Adults with Type 2 Diabetes. N Engl J Med. 2012;366(13):1209-17.

17. Kuna ST, Reboussin DM, Borradaile KE, Sanders MH, Millman RP, Zammit G, et al. Long-term effect of weight loss on obstructive sleep apnea severity in obese patients with type 2 diabetes. Sleep. 2013;36(5):641-649A.

18. Wing RR, Bond DS, Gendrano 3rd IN, Wadden T, Bahnson J, Lewis CE, et al. Effect of intensive lifestyle intervention on sexual dysfunction in women with type 2 diabetes: results from an ancillary Look AHEAD study. Diabetes Care. 2013;36(10):2937-44.

19. Faulconbridge LF, Wadden TA, Rubin RR, Wing RR, Walkup MP, Fabricatore AN, et al. One-Year Changes in Symptoms of Depression and Weight in Overweight/Obese Individuals With Type 2 Diabetes in the Look AHEAD Study. Obesity. 2012;20(4):783-93.

20. Baum A, Scarpa J, Bruzelius E, Tamler R, Basu S, Faghmous J. Targeting weight loss interventions to reduce cardiovascular complications of type 2 diabetes: a machine learning-based posthoc analysis of heterogeneous treatment effects in the Look AHEAD trial. Lancet Diabetes Endocrinol. 2017;5(10):808-15.

21. Gerstein HC, Miller ME, Genuth S, Ismail-Beigi F, Buse JB, Goff DCJ, et al. Long-term effects of intensive glucose lowering on cardiovascular outcomes. N Engl J Med. 2011;364(9):818-28.

22. Calles-Escandón J, Lovato LC, Simons-Morton DG, Kendall DM, Pop-Busui R, Cohen RM, et al. Effect of Intensive Compared With Standard Glycemia Treatment Strategies on Mortality by Baseline Subgroup Characteristics. Diabetes Care. 2010;33(4):721-7.

23. Miller ME, Bonds DE, Gerstein HC, Seaquist ER, Bergenstal RM, Calles-Escandon J, et al. The effects of baseline characteristics, glycaemia treatment approach, and glycated haemoglobin concentration on the risk of severe hypoglycaemia: post hoc epidemiological analysis of the ACCORD study. BMJ. 2010;340:b5444.

24. Genuth S, Ismail-Beigi F. Clinical implications of the ACCORD trial. J Clin Endocrinol Metab. 2012;97(1):41-8. 


\section{Supplementary Material}

Supplemental Table 1. Patient characteristics

\section{Look AHEAD \\ participants}

$(n=4,901)$

Demographics

Age (years)

$59 \pm 7$

Male sex

$2030(41 \%)$

Current smoking

$208(4 \%)$

Ethnicity

White / Caucasian

$3247(66 \%)$

Black / African-American

$804(16 \%)$

Hispanic

$676(14 \%)$

Other

$174(4 \%)$

Medical history

History of cardiovascular disease

690 (14\%)

History of myocardial infarction

$301(6 \%)$

History of stroke

$119(2 \%)$

Use of insulin

$832(17 \%)$

Duration of diabetes (years), median (IQR)

$5(2-10)$

Physical examination

Weight ( $\mathrm{kg}$ )

$101 \pm 19$

Body mass index $(\mathrm{kg} / \mathrm{m} 2)$

$36 \pm 6$

Waist circumference $(\mathrm{cm})$

$114 \pm 14$

Systolic blood pressure $(\mathrm{mmHg})$

$129 \pm 17$

Laboratory tests

Glycated hemoglobin $(\mathrm{mmol} / \mathrm{mol})$

$56 \pm 13$

Total cholesterol $(\mathrm{mmol} / \mathrm{L})$

$5.0 \pm 1.0$

High-density lipoprotein cholesterol ( $\mathrm{mmol} / \mathrm{L})$

$1.1 \pm 0.3$

Low-density lipoprotein cholesterol ( $\mathrm{mmol} / \mathrm{L})$

$2.9 \pm 0.8$

Triglycerides ( $\mathrm{mmol} / \mathrm{L})$

$2.0 \pm 1.3$

Creatinine $(\mu \mathrm{mol} / \mathrm{L})$, median $(\mathrm{IQR})$

$80(71-97)$

Presence of albuminuria

799 (17\%)

Presence of micro-albuminuria

$664(14 \%)$

Presence of macro-albuminuria

$135(3 \%)$

All values are presented as either $n(\%)$ or mean \pm SD unless noted otherwise 
Supplemental Table 2: Estimation of major cardiovascular events (myocardial infarction, stroke, hospitalization for angina, or cardiovascular mortality) for individual patients

Risk of major cardiovascular events

Formula

Linear predictor

\begin{tabular}{|c|}
\hline$\left(1-0.845^{\wedge}(\exp (\mathbf{A}-3.191))\right) * 100 \%$ \\
\hline $\mathbf{A}=0.038 *$ (age) $-0.005 *$ (age if treated with intervention) $+0.552 *$ (if male) - \\
\hline treated with intervention $)+0.165 *(i$ \\
\hline treated with intervention $)+0.007 *(\mathrm{SBP} i$ \\
\hline in $\mathrm{mmHg}$ if treated with intervention) -0.246 * (non-HDL in $\mathrm{mmol} / \mathrm{L})+0.015 *$ (non- \\
\hline $\mathrm{HDL}$ in $\mathrm{mmol} / \mathrm{L}$ if treated with intervention $)+0.053 *$ (non- $\mathrm{HDL}^{2}$ in $\left.\mathrm{mmol} / \mathrm{L}\right)-0.007$ \\
\hline * $\left(\right.$ non- $\mathrm{HDL}^{2}$ in $\mathrm{mmol} / \mathrm{L}$ if treated with intervention $)+0.003$ * $(\mathrm{HbA} 1 \mathrm{c}$ in $\mathrm{mmol} / \mathrm{mol})$ \\
\hline$+0.019 *(\mathrm{HbA} 1 \mathrm{c}$ in $\mathrm{mmol} / \mathrm{mol}$ if treated with intervention $)+0.0005 *(\mathrm{BMl}$ in $\mathrm{kg} /$ \\
\hline $\mathrm{g} / \mathrm{m}^{2}$ if treated with intervention) -0.006 * (CKD-EPI in $\mathrm{ml} /$ \\
\hline $\mathrm{min} / 1.73)-6.850 \mathrm{E}-5$ * (CKD-EPI in $\mathrm{ml} / \mathrm{min} / 1.73$ if treated with intervention $)+0.288$ \\
\hline inuria) -0.320 * (if micro-albun \\
\hline+0.136 * (if macro-albuminuria) +0.258 * (if macro-albuminuria if treated with \\
\hline intervention $)+0.016 *$ (duration of diabetes \\
\hline in years if treated with intervention) +1.041 * (if history of CVD) +0.316 * (if history \\
\hline of CVD if treated with intervention) -0.002 * (if insulin user) +0.036 * (if insulin user \\
\hline treated with intervention) +0.016 * (if treated with intervention) \\
\hline
\end{tabular}

$\overline{S B P}=$ systolic blood pressure; non-HDL = non-high density lipoprotein cholesterol; $H b A 1 c=$ glycated hemoglobin $A 1 c ; B M I=$ body mass index; $C K D-E P I=$ glomerular filtration rate (estimated with the CKD-EPI formula); $C V D$ = cardiovascular disease 
Supplemental Figure 1: Internal validation of the major cardiovascular events (MACE) risk model: Calibration plot of agreement between estimated and observed 10-year risk of MACE in the Look AHEAD study population, and associated C-statistic.

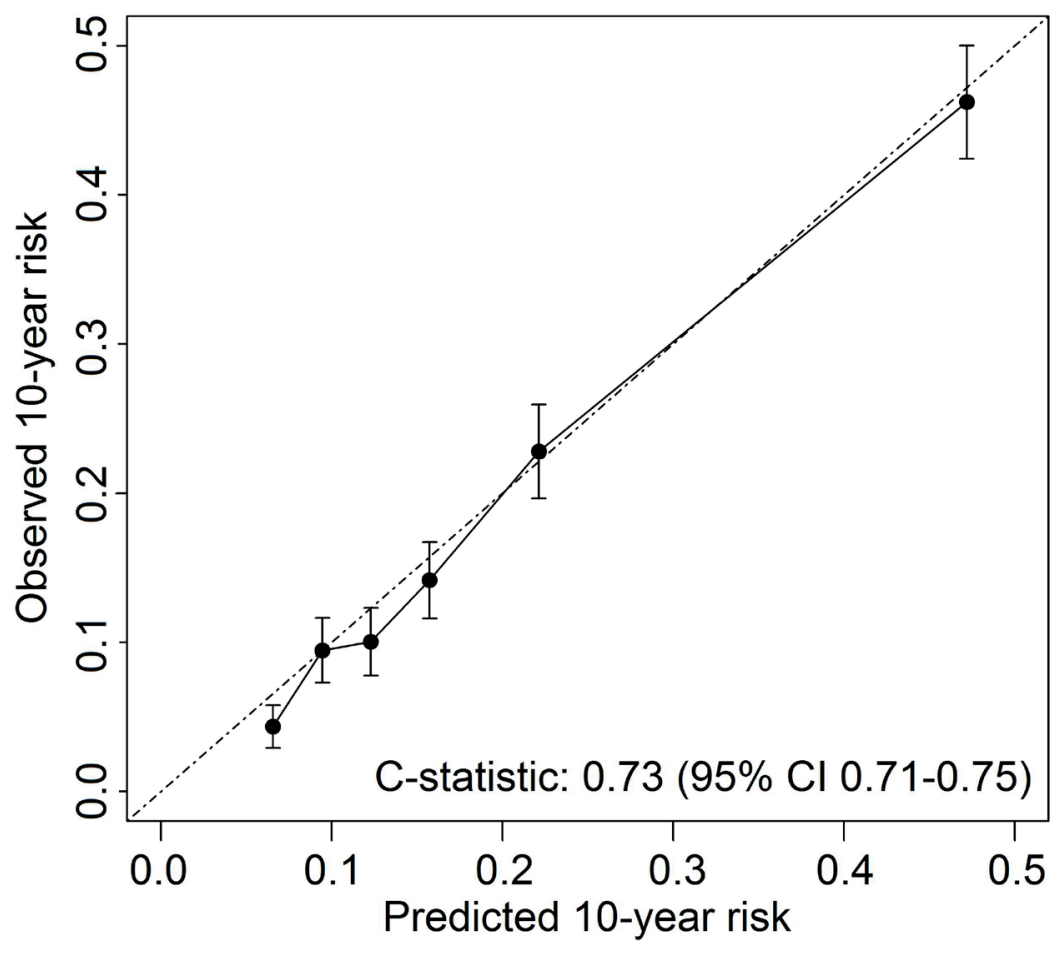


CHAPTER 9 | Heterogeneity of treatment effects from an intensive lifestyle intervention 


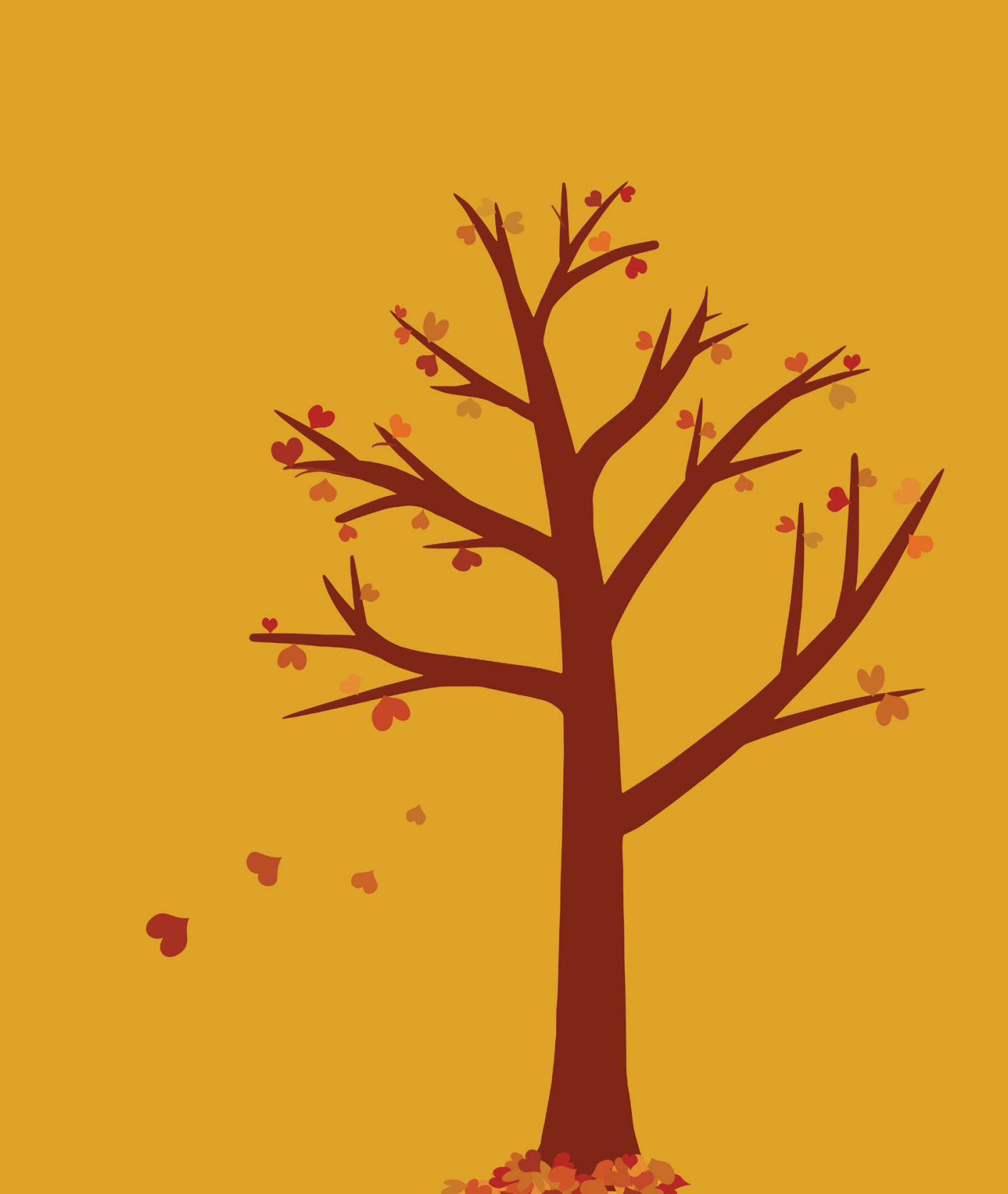



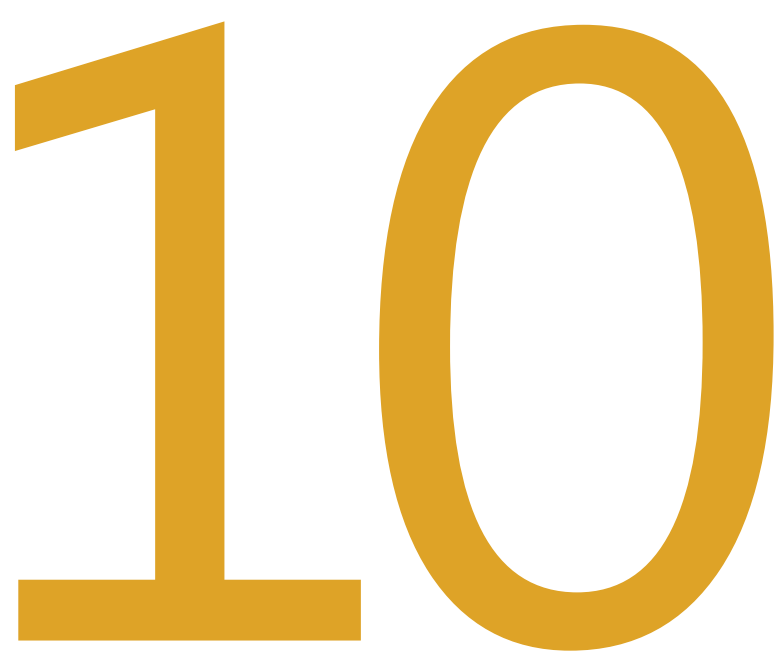

General Discussion 
Cardiovascular disease (CVD) remains a leading cause of morbidity and mortality worldwide, despite implementation of international guidelines focused on management of the most important amendable risk factors for CVD. Many observational studies and randomized controlled trials have demonstrated the beneficial effects of smoking cessation, ${ }^{1}$ lipid lowering, ${ }^{2}$ blood pressure lowering, ${ }^{3}$ antithrombotic therapy, ${ }^{4}$ and glucose lowering. ${ }^{5}$ As cardiovascular risk management is associated with a high burden on the health care system, there is a need to identify those patients who have the highest risk of CVD to be able to effectively treat those patients who are likely to benefit from those preventive treatment strategies. Therefore, risk assessment is the cornerstone in current international guidelines. ${ }^{6,7}$ However, the paradigm of "high risk equals high benefit" is under debate.

\section{Residual risk of cardiovascular disease}

Traditional modifiable risk factors, such as smoking, blood pressure, and cholesterol, are associated with the cumulative event burden in patients with established CVD (chapter 2), demonstrating the importance of adequate risk factor control in patients with established CVD.

Although blood pressure-lowering, lipid-lowering, and smoking cessation have been the cornerstone of cardiovascular risk management in for decades, ${ }^{8,9}$ high-risk patients, especially those with established CVD or with type 2 diabetes, continue to experience (recurrent) CVD. ${ }^{10}$ This concept has been dubbed 'residual risk'.11

\section{Novel risk factors for cardiovascular disease}

There is ongoing interest to find novel potential drivers of (residual) CVD risk. A plethora of biomarkers have been studied in relation to the risk of CVD. These can be causally related risk factors involved in atherosclerosis, but non-causally related biomarkers can also be of clinical importance if they can improve CVD risk stratification to improve clinical decision making. ${ }^{12}$

Biomarkers that have been found to be independently associated with CVD include, among many others, inflammation markers, such as C-reactive protein ${ }^{13}$ and interleukin-6; ${ }^{14}$ lipidrelated biomarkers, such as lipoprotein $(a)^{15}$ and apolipoprotein $\mathrm{B}_{i^{16}}$ and other biomarkers, such as pro-N-terminal brain natriuretic peptide ${ }^{17}$ and high-sensitivity cardiac troponin. ${ }^{18,19}$ Another potential risk factor of interest is the thyroid function. Even within what is currently considered to be the 'normal range', thyroid-stimulating hormone (TSH) has been found to be associated with myocardial infarction, ${ }^{20}$ mortality, ${ }^{21,22}$ and stroke. ${ }^{23}$ In patients with type 2 diabetes, higher TSH within the normal range was found to be related to a lower risk of stroke (chapter $\mathbf{3}$ ). This is of interest, as type 2 diabetes and thyroid function are closely 
related. ${ }^{24,25}$ It has previously even been suggested that TSH levels, even within the normal range, are related to the risk of incident type 2 diabetes, however, these findings were not supported by the cohort study and meta-analysis presented in this thesis (chapter 4). Thus, TSH levels are associated with CVD, even in what is currently considered the normal range. There have been ongoing discussions in the literature whether the reference range for TSH needs to be changed to reflect these findings. ${ }^{26}$ However, this would lead to the re-classification of many people, including many healthy people, to 'abnormal' thyroid function, while it is yet unknown how this what the consequence of this would be for (healthy) individual patients in clinical practice. Despite the evidence that thyroid function is a risk factor for CVD, there is of yet no evidence that thyroid hormone replacement has important health benefits outside of overt thyroid dysfunction. ${ }^{27,28}$ As such, these findings, for now, carry no clinical consequences. TSH might prove to improve CVD risk stratification, however, this should be assessed in prognostic studies.

\section{Treatment choices for reducing residual risk}

Knowledge of (novel) drivers of residual risk may lead to new treatment targets. There have been major advances in addressing this residual risk in the last few years. As lipids remain an important risk factor for recurrent events in patients with established CVD (as also shown in chapter $\mathbf{2}$ ), the newest guidelines have set even more stringent treatment goals, with a target for low density lipoprotein $(L D L)$ cholesterol of $1.4 \mathrm{mmol} / \mathrm{L}$ in the newest dyslipidaemia guideline from the European Society of Cardiology (ESC), compared to $1.8 \mathrm{mmol} / \mathrm{L}$ in the previous guideline. ${ }^{29,30}$ These very low levels can be achieved with new lipid-lowering treatments, for example with proprotein convertase subtilisin/kexin type 9 (PCSK9) inhibitors. ${ }^{31,32}$

Other recent advancements have been the development of anti-inflammatory therapies to address the residual inflammatory risk, ${ }^{33,34}$ the addition of low-dose rivaroxaban to aspirin for more intensive antithrombotic therapy, ${ }^{35}$ and new therapies in the management of type 2 diabetes, such as sodium-glucose transport protein 2 (SGLT2) inhibitors and glucagonlike peptide-1 (GLP-1) analogues. ${ }^{36,37}$

\section{Making the right treatment choices}

With the many different possible treatment options now available to further lower cardiovascular risk, it can be difficult to know what the best choice is for an individual patient in clinical practice. Should we lower their blood pressure, or their lipids, or maybe both? And how much should we lower them? Do we need to add PCSK9 inhibitors, because "the lower LDL-cholesterol the better"? ${ }^{29}$ Will they benefit from (intensified) antithrombotic therapy? Is this patient perhaps a candidate for an anti-inflammatory therapy? Will SGLT2 
inhibition or a GLP-1 agonist improve the prognosis of this patient with type 2 diabetes? Besides having a risk of adverse events, some of these therapies are associated with high costs, which may be important on a healthcare economic perspective, especially if not given to the 'right' patients.

\section{Using risk stratification for treatment decisions}

Luckily, international guidelines are available to provide guidance in making these treatment decisions. As stated in the general introduction of this thesis, the prevalent paradigm is that patients are treated based on their risk of future events. Depending on the risk category, there are certain treatment threshold and treatment targets for pharmacotherapy. In primary prevention, future (10-year) risk is determined using individualized risk stratification with multivariate risk models. Patients with established CVD are all assumed to be at (very) high risk of future cardiovascular events, as are patients with type 2 diabetes. However, it has been demonstrated that not all patients with established vascular disease are at high future risk of $C V D,{ }^{40}$ and the same is true for patients with type 2 diabetes. ${ }^{41}$ The current one size fits all risk-based guidelines for high risk patients may therefore be rather too simplistic, and risk prediction may have an important place in a more individualized approach to make personalized treatment decisions for (secondary) prevention of CVD.

\section{Risk prediction in different patient populations}

Although individualized risk stratification using multivariate risk models is currently only widely recommended for primary prevention, using e.g. SCORE (in Europe) or ASCVD in the USA, ${ }^{6,7}$ risk models predicting future (10-year) CVD risk have already been developed and made available as online calculators for several different patient populations. These include e.g. the SMART score and TRS $2^{\circ} \mathrm{P}$ risk score for patients with established CVD; ${ }^{42,43}$ the ADVANCE and UKPDS risk engines for patients with type 2 diabetes; ${ }^{44,45}$ and SCOREO.P. and an older-person specific risk score derived in the PROSPER trial for older persons (chapter 6). ${ }^{46,47}$

Prediction models for specific populations may include predictors that are specifically aimed at these populations. For example, although several risk models for patients without CVD also include the presence of diabetes as a predictor, using a model targeted at patients with diabetes specifically will likely result in more accurate estimations, as diabetes-specific risk factors (such as $\mathrm{HbA1c}$ levels and the duration of diabetes) are taken into consideration. Risk prediction in older persons requires risk models derived in an older population, as the relationship between risk factors and CVD attenuates with age.48 Furthermore, an older person-specific risk score should be adjusted for the competing risk of non-CVD mortality. Failing to do so leads to overestimations of CVD risk in an older 
population, in whom non-CVD mortality becomes increasingly important. ${ }^{49}$ The older person-specific risk score presented in this thesis takes these factors into account, and reliably estimates risk in older persons (chapter 6).

However, an additional problem in older persons is that even with a reliable risk estimations, the vast majority will have high 10-year risks of CVD. But does that mean that we should treat all these elderly with preventive therapy? Or does that mean that all older persons will even really benefit from cardiovascular risk management? Especially if an individual patient also has very high risk of non-CVD mortality, the benefit may be very limited. In other words, we might need another approach than 10-year risk to properly discriminate between patients who will and will not benefit importantly from preventive (pharmaco) therapy. Thankfully, recent methodological advancements that allow estimations of risk on a lifetime perspective may provide an answer for this problem.

\section{Lifetime risk estimations}

As age is the most important driver of 10 -year CVD risk, older persons are invariably at higher risk than younger persons. Based on 10-year risk thresholds for treatment decisions, such as the ones established for SCORE in the ESC/EAS guidelines, ${ }^{6}$ older persons may have very high 10-year risks, but a relatively limited life expectancy over which a treatment can exert a beneficial effect. At the same time, younger patients are usually not eligible for preventive pharmacotherapy.

It is now possible to estimate future risk in a lifetime perspective, expressed as either a percentage lifetime CVD risk, or event-free life expectancy. ${ }^{50}$ Currently available prediction models for CVD include JBS-3 and LIFE-CVD for primary prevention, ${ }^{50,51}$ SMART-REACH for secondary prevention, ${ }^{52}$ and DIAL for patients with type 2 diabetes. ${ }^{53}$ As younger patients have a relatively longer remaining life expectancy, their lifetime risk of developing CVD is higher than older persons with similar risk factor levels (chapter 7). As such, this new methodology can change the way we look at which patients are 'at high risk of future CVD', shifting away from the traditional high 10-year risk approach. Additionally, the new lifetime methodology can be used to go even a step further in a personalized approach, as will be explained in the next paragraph.

\section{Going from risk stratification to treatment effect estimation}

The current guidelines use risk stratification to guide the decision-making process. An alternative method to decide which patients should be treated, and with what therapy, is to directly look at treatment benefit rather than risk. After all, the use of risk stratification is meant as a way to identify those patients who will have the highest benefit, under the assumption that "high risk equals high benefit". 
Assuming that the relative treatment effect is the same for all patients (chapter $\mathbf{5}$ ), results from either single randomized clinical trials or meta-analyses can be combined with multivariate risk models to estimate the absolute treatment effect from therapies (e.g. 10year absolute risk reduction [ARR], or lifetime benefit in terms of 'increased life expectancy free from $\left.C V D^{\prime}\right) .{ }^{54,55}$ This methodology can be used to predict treatment effects from a wide range of therapy options, including e.g. lipid lowering, blood pressure-lowering, antithrombotic treatment, and glucose lowering. ${ }^{51,53,56-58}$ We have included an example of this methodology in this thesis in chapter $\mathbf{6}$, by estimating the treatment effect of blood pressure lowering for individual older persons using the older person-specific risk score. There is a wide distribution in estimated 10 -year absolute risk reduction from blood pressure lowering, which is not only dependent on the baseline ('pre-treatment') predicted future CVD risk, but also on the blood pressure at baseline. These results demonstrate the importance of individualized treatment effect predictions for making treatment decisions - those with the highest future risk are not necessarily the same who have high benefit, especially when their risk is not primarily driven by blood pressure.

Using lifetime benefit rather than 10-year risk gives a shift of patients benefitting most from treatment from older persons towards younger persons with higher risk factor levels (chapter 7). Thus, lifetime benefit estimations can help with the following clinical conundrum: young patients are under the current definitions usually considered at low risk even in the presence of significant risk factors, and therefore do not have an indication for preventive pharmacological interventions, even though atherosclerotic disease is the result of life-long exposure to these risk factors. Under the risk-based approach, risk factor modification may be unjustly delayed in younger patients with high lifetime risk, while there is potential overtreatment in older persons with high short-term risk but limited benefit (especially in patients with high risk of competing events or with limited remaining life expectancy). Being able to estimate benefit might thus lead to a shift in paradigm, where "high risk equals high benefit" is no longer always considered to be true, and preventive strategies are aimed at maximizing benefit instead of treating those at highest risk.

\section{Predicting adverse effects}

In shared-decision making it is important to not only consider the return on investment from (potentially lifelong) preventive pharmacotherapy, but also to weigh the benefit against potential harms. Although some considerations against initiation (or intensification) of therapy may be of a subjective nature (e.g. the perceived burden of having to take daily medication, past experiences, experiences from family or friends), it may be possible to quantify some of the harms for individual patients using multivariate risk assessment. Examples of harms that may be quantified for individual patients include the risk of severe hypoglycemia from intensive glucose control in patients with type 2 diabetes, ${ }^{57}$ or the risk of major bleeding with antithrombotic therapy. ${ }^{58}$ 
The individual treatment effects from therapy on the risk of adverse events may also be estimated on a lifetime perspective, as illustrated in chapter $\mathbf{8}$ of this thesis. Adding a lowdose direct oral anticoagulant (DOAC) to aspirin for patients with stable cardiovascular disease is one of the newer developments to target residual risk in secondary prevention. ${ }^{35}$ Lifetime treatment effects from this therapy can be estimated for both the cardiovascular benefit (using the SMART-REACH model available on www.U-Prevent.com) ${ }^{52}$ and the bleeding harm (using a newly derived major bleeding lifetime model). Figure 1 illustrates how these estimations can be obtained for an individual patient in clinical practice. These treatment effect estimations of benefit versus harm can then be weighed in clinical decision making for this individual patient. Cardiovascular benefit outweighs bleeding harm in the majority of patients (chapter 8 ), indicating that low-dose rivaroxaban may be

Figure 1. What are the lifetime benefit and lifetime harm of adding low-dose rivaroxaban to aspirin for an individual patient? A patient example.

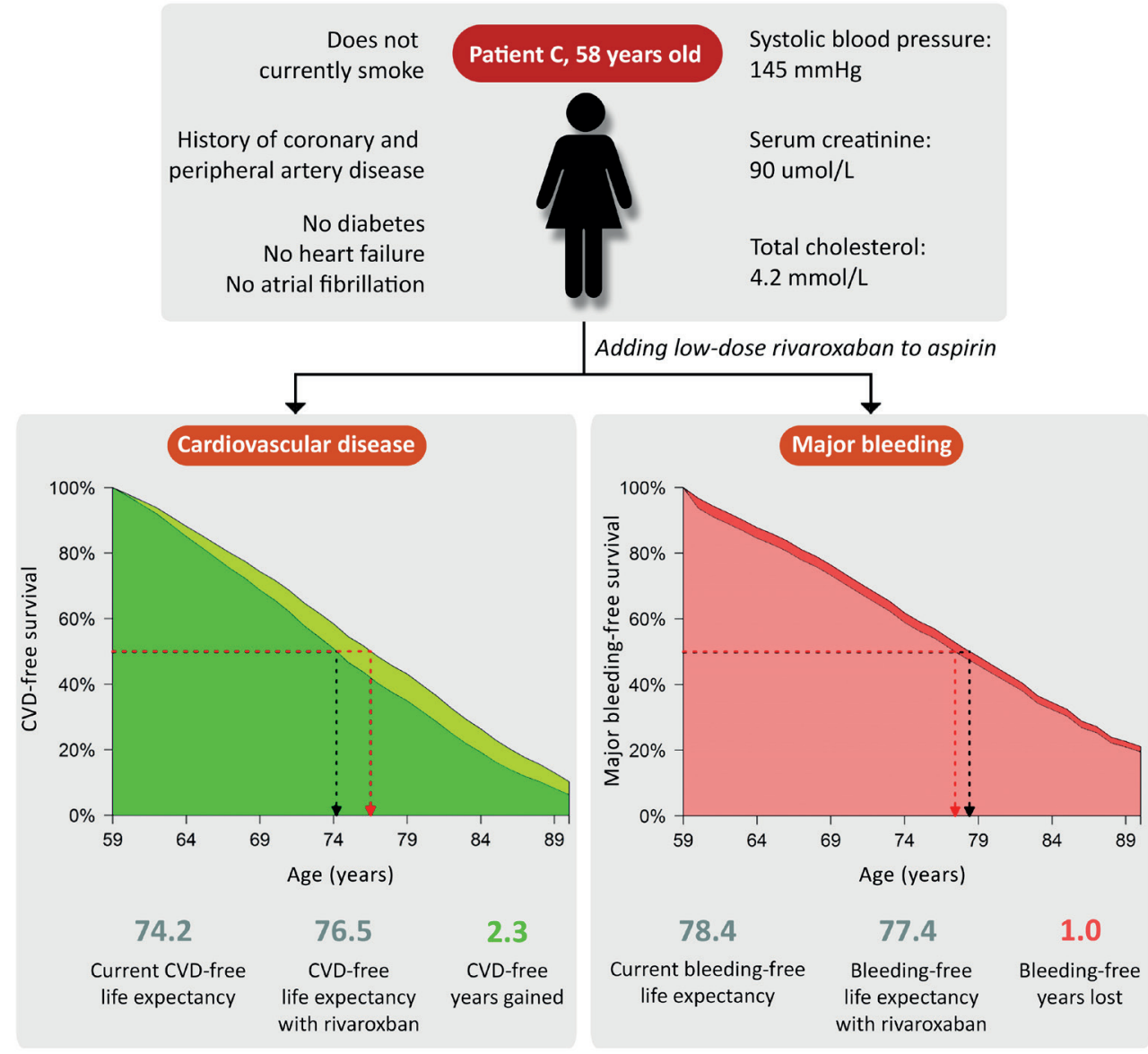


a suitable new therapy for further reducing residual CVD risk in a major part of patients in secondary prevention, although what is considered to be 'enough' lifetime benefit for treatment to be warranted should be discussed by patients, doctors and governments. ${ }^{59}$

\section{Methodological considerations for treatment effect estimation}

It was previously mentioned that risk models can be combined with hazard ratios to estimate treatment effects, under the assumption that the relative treatment effect is the same for all patients. However, the relative treatment effect may in reality not be uniform across a population and may differ in terms of benefits and important side effects (i.e. relative heterogeneity of treatment effects may be present). Therefore, this assumption needs to be checked before this methodology can be applied. There are multiple methods available for checking this assumption. ${ }^{60}$

While subgroup analyses are the most well-known analyses to assess heterogeneity of treatment effects, there are multiple disadvantages. ${ }^{61}$ An alternative method is to assess whether the baseline risk - as assessed with multivariate risk estimation - of individual patients in a trial is associated with the relative treatment effect. Heterogeneity of treatment effect can be assessed in trial data using Cox models including trial allocation, baseline risk as predicted with a multivariate risk model, and their interaction term as covariates. If the interaction term is statistically significant, this indicates that the relative trial effect is dependent on baseline risk. In that case, the single hazard ratio reported by a trial cannot be applied to all patients, and a more personalized approach is necessary to determine treatment effects of the respective therapy for individual patients (chapter 5). An alternative method to capture treatment effect heterogeneity on the relative scale is through the inclusion of treatment allocation-by-covariate interaction terms in a multivariate risk model derived in an RCT. ${ }^{60,62}$ This makes it possible to directly estimate the treatment effect for individual patients. Stratification by estimated treatment effect, rather than by risk, can then be used for clinical treatment decision making. As an example of this methodology, in chapter 9 of this thesis, such a model was developed to assess relative heterogeneity of treatment effects in the Action for Health in Diabetes (Look AHEAD) trial. This trial found, on average, no effect of an intensive lifestyle intervention aimed at weight loss in overweight and obese patients with type 2 diabetes on cardiovascular outcomes in the primary analysis. ${ }^{63}$ Stratification based on the treatment effect, estimated using a multivariate risk model including treatment-by-covariate interaction terms derived in the trial, showed that this intervention may reduce cardiovascular events in selected patients, but may have a detrimental treatment effect in others. This finding is thought-provoking and clinically important, as it has always been assumed that lifestyle interventions are relatively harmless, and are currently recommended for all patients with diabetes. ${ }^{6,64,65}$ We propose that effect modification of the relative treatment effect should be assessed 
systematically in all RCTs using multivariate risk prediction, in terms of both treatment efficacy and important treatment harms, as a pre-specified analysis. Relative treatment effects can then be translated more reliably to individual absolute treatment effects which can be weighed for individualized clinical decision making.

\section{Future perspectives in CVD prediction}

It is clear that using CVD prediction tools in multiple patient populations as decision support tools may be instrumental in finding the best balance between benefit and disadvantages for individual patients, and in effectively allocating available clinical resources.

The ultimate goal of CVD prediction tools is to distinguish between those patients who will and will not benefit importantly from (specific) preventive strategies. It should be considered to extend the use of CVD prediction tools from just middle-aged persons without CVD to many different patient populations, including patients with established CVD or with diabetes. Lifetime predictions models can improve personalized prevention strategies in patient populations where 10-year risk models cannot properly distinguish between those who will and will not benefit from treatment, such as younger or older persons (chapter 7). This will personalize CVD prevention in these patient categories in a way that is not done in the current international guidelines.

However, there are still many questions and concerns that need to be answered. First, there is, for now, no clear precedent in how to interpret predictions from different risk models. While thresholds for treatment decisions exist for e.g. SCORE and ASCVD, this is not true for all available risk models, and certainly not in terms of (lifetime) benefit estimations. Further research is necessary to establish which thresholds have the best balance in terms of benefits versus harms, and in terms of cost-effectiveness at both a group and a more individualized level.

Secondly, more research is needed to establish how therapy benefit can be most effectively communicated with patients. Lifetime treatment effect estimations - e.g. "one year of life expectancy without myocardial infarction or stroke gained"- are more intuitive than the percentages that are estimated with 10 -year risk models, ${ }^{66}$ This may improve the communication of therapy benefits in clinician-patient communication, improving shareddecision making. As lifetime benefit is an easy to interpret concept, it may also help in patient involvement in shared decision making and patient motivation for preventive measures. ${ }^{67}$ However, there may be patients for whom absolute or relative risk reductions are easier to understand and weigh, and therefore still more effective.

Thirdly, cost-effectiveness analyses are necessary to compare an individualized, benefitbased approach with the risk-based approach currently used in the guidelines. A potential disadvantage of a lifetime approach is that persons will be eligible for treatment at a much 
younger age, increasing the average treatment duration and thus the lifetime treatment costs.

Fourthly, there are some potential obstacles in implementation of CVD prediction tools in clinical practice. An important objection that clinicians may have against routine use of risk models in their daily practice, is that it may be experienced as time-consuming. Although the use of risk stratification will likely be more efficient long-term, in terms of improvement in decision-making and potentially even in reduction of CVD morbidity and mortality, this is an important obstacle in the implementation. Therefore, future innovation can help with incorporating risk prediction tools into electronic health records, so that predictor information can be automatically filled in, saving a lot of time.

Finally, an important assumption that is made in lifetime treatment effect modelling is that the relative treatment effect of preventive medication remains constant over long-term follow-up. Mendelian randomization studies have shown that lifelong genetic exposure to lower SBP and LDL was associated with a lower cardiovascular risk, with a magnitude larger than expected from the combined treatment effects from pharmacological risk factor reduction. ${ }^{68}$ Although these findings cannot be assumed to represent the potential benefit achievable with risk factor reduction, they may indicate that risk factor treatment from a very young age may have an even higher lifetime benefit than estimated currently.

\section{Concluding remarks}

Residual risk for CVD remains an important problem in high-risk patients. Many biomarkers have been studied that are either causally or prognostically associated with the risk of CVD. However, CVD prediction tools should preferably include clinically readily available patient characteristics to be able to be easy to use in clinical practice. Using CVD prediction tools in different patient populations, including those considered (very) high-risk in the current international guidelines, allows a more individualized approach of tackling the issue of residual risk. Going from a risk-based approach to a benefit-based approach may further personalize and improve individualized CVD prevention. Estimations of treatment benefit can be weighed with the potential risks and costs. Recent methodological developments that allow us to estimate lifetime treatment effects may help in clinicianpatient communication, and thus improve shared decision making and patient motivation. Especially in both the younger and the older persons, in whom 10-year risk estimations fall short to make personalized treatment decisions, lifetime treatment effects have the chance to improve personalized CVD prevention. (Lifetime) prediction models for several different patient populations are freely available on www.U-Prevent.com. These prediction tools allow us to choose the right therapy for the right patient. To conclude, although we still cannot truly look into our patients' futures, we are trying our hardest. 


\section{Highlights of this thesis}

- In patients with a recent first manifestation of cardiovascular disease, the cardiovascular risk factors smoking, non-high density lipoprotein cholesterol, and systolic blood pressure are not only independently associated with the risk a first recurrent event during follow-up, but with the total cardiovascular event burden during long-term follow-up. (Chapter 2)

- Thyroid stimulating hormone levels in the normal range do not influence the risk of incident type 2 diabetes. In patients with established diabetes, higher thyroid stimulating hormone levels in the normal range give a reduced risk of future stroke, but are not associated with other cardiovascular disease. (Chapters 3 and 4)

- Effect modification of the relative treatment effect of preventive medication based on the baseline risk of future events, as estimated with multivariate risk modelling, can be assessed in RCTs with a survival model including an interaction term between baseline risk and treatment allocation (Chapter 5)

- An older person-specific risk score can be used to reliably predict 10-year risk of CVD in individual persons aged 65 or older, and to estimate the absolute treatment benefit from blood pressure lowering (Chapter 6)

- Treatment decisions for risk factor modification based on estimated lifetime benefit rather than estimated absolute 10-year risk may lead to the treatment of younger high-risk patients, rather than older patients, in the primary prevention of CVD (Chapter 7)

- Using lifetime prediction models, lifetime cardiovascular benefit and bleeding harm from adding low-dose rivaroxaban to aspirin for patients with stable CVD can be estimated, which can be weighed to facilitate treatment decisions in clinical practice (Chapter 8)

- In a trial studying intensive lifestyle intervention aimed at weight loss in overweight and obese patients with type 2 diabetes, a multivariate risk model including treatment-by-covariate interaction terms showed that this intervention may reduce cardiovascular events in selected patients, but may have a detrimental treatment effect in others (Chapter 9) 


\section{References}

1. Jha P, Ramasundarahettige $C$, Landsman V, Rostron B, Thun M, Anderson RN, et al. 21stcentury hazards of smoking and benefits of cessation in the United States. N Engl J Med. 2013;368(4):341-50.

2. Mihaylova B, Emberson J, Blackwell L, Keech A, Simes J, Barnes EH, et al. The effects of lowering LDL cholesterol with statin therapy in people at low risk of vascular disease: meta-analysis of individual data from 27 randomised trials. Lancet. 2012;380(9841):581-90.

3. Ettehad D, Emdin CA, Kiran A, Anderson SG, Callender T, Emberson J, et al. Blood pressure lowering for prevention of cardiovascular disease and death: a systematic review and metaanalysis. Lancet. 2016;387(10022):957-67.

4. Collaboration AT (ATT), Baigent C, Blackwell L, Collins R, Emberson J, Godwin J, et al. Aspirin in the primary and secondary prevention of vascular disease: collaborative meta-analysis of individual participant data from randomised trials. Lancet. 2009;373(9678):1849-60.

5. Turnbull FM, Abraira C, Anderson RJ, Byington RP, Chalmers JP, Duckworth WC, et al. Intensive glucose control and macrovascular outcomes in type 2 diabetes. Diabetologia. 2009;52(11):88-98

6. Piepoli MF, Hoes AW, Agewall S, Albus C, Brotons C, Catapano AL, et al. 2016 European Guidelines on cardiovascular disease prevention in clinical practice. Eur Heart J. 2016;37(29):2315-81.

7. Goff DCJ, Lloyd-Jones DM, Bennett G, Coady S, D'Agostino RB, Gibbons R, et al. 2013 ACC/ AHA Guideline on the Assessment of Cardiovascular Risk: A Report of the American College of Cardiology/American Heart Association Task Force on Practice Guidelines. J Am Coll Cardiol. 2014;63(25):S49-73.

8. Pyorala K, De Backer G, Graham I, Poole-Wilson P, Wood D. Prevention of coronary heart disease in clinical practice. Recommendations of the Task Force of the European Society of Cardiology, European Atherosclerosis Society and European Society of Hypertension. Eur Heart J. 1994;15(10):1300-31.

9. The recognition and management of hyperlipidaemia in adults: A policy statement of the European Atherosclerosis Society. Eur Heart J. 1988;9(5):571-600.

10. Engelen SE, van der Graaf Y, Stam-Slob MC, Grobbee DE, Cramer MJ, Kappelle LJ, et al. Incidence of cardiovascular events and vascular interventions in patients with type 2 diabetes. Int J Cardiol. 2017;248:301-7.

11. Matsuura Y, Kanter JE, Bornfeldt KE. Highlighting Residual Atherosclerotic Cardiovascular Disease Risk. Arterioscler Thromb Vasc Biol. 2019;39(1):e1-9.

12. Lacey B, Herrington WG, Preiss D, Lewington S, Armitage J. The Role of Emerging Risk Factors in Cardiovascular Outcomes. Curr Atheroscler Rep. 2017;19(6):28.

13. Kaptoge S, Di Angelantonio E, Lowe G, Pepys MB, Thompson SG, Collins R, et al. C-reactive protein concentration and risk of coronary heart disease, stroke, and mortality: an individual participant meta-analysis. Lancet. 2010;375(9709):132-40.

14. Sarwar N, Butterworth AS, Freitag DF, Gregson J, Willeit P, Gorman DN, et al. Interleukin-6 
receptor pathways in coronary heart disease: a collaborative meta-analysis of 82 studies. Lancet. 2012;379(9822):1205-13.

15. Erqou S, Kaptoge S, Perry PL, Di Angelantonio E, Thompson A, White IR, et al. Lipoprotein(a) concentration and the risk of coronary heart disease, stroke, and nonvascular mortality. J Am Med Assoc. 2009;302(4):412-23.

16. Boekholdt SM, Arsenault BJ, Mora S, Pedersen TR, LaRosa JC, Nestel PJ, et al. Association of LDL cholesterol, non-HDL cholesterol, and apolipoprotein B levels with risk of cardiovascular events among patients treated with statins: a meta-analysis. JAMA. 2012;307(12):1302-9.

17. Di Angelantonio E, Chowdhury R, Sarwar N, Ray KK, Gobin R, Saleheen D, et al. B-type natriuretic peptides and cardiovascular risk: systematic review and meta-analysis of 40 prospective studies. Circulation. 2009;120(22):2177-87.

18. Sze J, Mooney J, Barzi F, Hillis GS, Chow CK. Cardiac Troponin and its Relationship to Cardiovascular Outcomes in Community Populations - A Systematic Review and Meta-analysis. Heart Lung Circ. 2016;25(3):217-28.

19. Willeit P, Welsh P, Evans JDW, Tschiderer L, Boachie C, Jukema JW, et al. High-Sensitivity Cardiac Troponin Concentration and Risk of First-Ever Cardiovascular Outcomes in 154,052 Participants. J Am Coll Cardiol. 2017;70(5):558-68.

20. Westerink J, van der Graaf Y, Faber DR, Spiering W, Visseren FLJ, SMART study group. Relation between thyroid-stimulating hormone and the occurrence of cardiovascular events and mortality in patients with manifest vascular diseases. Eur J Prev Cardiol. 2012;19(4):864-73.

21. Åsvold BO, Bjøro T, Platou C, Vatten LJ. Thyroid function and the risk of coronary heart disease: 12-year follow-up of the HUNT study in Norway. Clin Endocrinol. 2012;77(6):911-7.

22. Inoue K, Tsujimoto T, Saito J, Sugiyama T. Association Between Serum Thyrotropin Levels and Mortality Among Euthyroid Adults in the United States. Thyroid. 2016;26(10):1457-65.

23. Chaker L, Baumgartner C, den Elzen WPJ, Collet T-H, Ikram MA, Blum MR, et al. Thyroid Function Within the Reference Range and the Risk of Stroke: An Individual Participant Data Analysis. J Clin Endocrinol Metab. 2016;101(11):4270-82.

24. Wang C. The Relationship between Type 2 Diabetes Mellitus and Related Thyroid Diseases. J Diabetes Res. 2013;2013:390534.

25. Han C, He X, Xia X, Li Y, Shi X, Shan Z, et al. Subclinical Hypothyroidism and Type 2 Diabetes: A Systematic Review and Meta-Analysis. PLoS One. 2015;10(8):e0135233.

26. Biondi B. The Normal TSH Reference Range: What Has Changed in the Last Decade? J Clin Endocrinol Metab. 2013;98(9):3584-7.

27. Lamine F, De Giorgi S, Marino L, Michalaki M, Sykiotis GP. Subclinical hypothyroidism: new trials, old caveats. Hormones. 2018;17(2):231-6.

28. Stott DJ, Rodondi N, Kearney PM, Ford I, Westendorp RGJ, Mooijaart SP, et al. Thyroid Hormone Therapy for Older Adults with Subclinical Hypothyroidism. N Engl J Med. 2017;376(26):25342544.

29. Mach F, Baigent C, Catapano AL, Koskinas KC, Casula M, Badimon L, et al. 2019 ESC/EAS 
Guidelines for the management of dyslipidaemias: lipid modification to reduce cardiovascular risk. Eur Heart J. 2020;41(1):111-188.

30. Catapano AL, Graham I, De Backer G, Wiklund O, Chapman MJ, Drexel H, et al. 2016 ESC/EAS Guidelines for the Management of Dyslipidaemias. Eur Heart J. 2016 Oct 14;37(39):2999-3058.

31. Sabatine MS, Giugliano RP, Keech AC, Honarpour N, Wiviott SD, Murphy SA, et al. Evolocumab and Clinical Outcomes in Patients with Cardiovascular Disease. N Engl J Med. 2017;376(18):171322.

32. Schwartz GG, Steg PG, Szarek M, Bhatt DL, Bittner VA, Diaz R, et al. Alirocumab and cardiovascular outcomes after acute coronary syndrome. N Engl J Med. 2018;379(22):2097-107.

33. Ridker PM, Everett BM, Thuren T, MacFadyen JG, Chang WH, Ballantyne C, et al. Antiinflammatory therapy with canakinumab for atherosclerotic disease. N Engl J Med. 2017;377(12):1119-31.

34. Tardif J-C, Kouz S, Waters DD, Bertrand OF, Diaz R, Maggioni AP, et al. Efficacy and Safety of Low-Dose Colchicine after Myocardial Infarction. N Engl J Med. 2019;381(26):2497-505.

35. Eikelboom JW, Connolly SJ, Bosch J, Dagenais GR, Hart RG, Shestakovska O, et al. Rivaroxaban with or without Aspirin in Stable Cardiovascular Disease. N Engl J Med. 2017;377(14):1319-30.

36. Zelniker TA, Wiviott SD, Raz I, Im K, Goodrich EL, Bonaca MP, et al. SGLT2 inhibitors for primary and secondary prevention of cardiovascular and renal outcomes in type 2 diabetes: a systematic review and meta-analysis of cardiovascular outcome trials. Lancet. 2019;393(10166):31-9.

37. Kristensen SL, Rørth R, Jhund PS, Docherty KF, Sattar N, Preiss D, et al. Cardiovascular, mortality, and kidney outcomes with GLP-1 receptor agonists in patients with type 2 diabetes: a systematic review and meta-analysis of cardiovascular outcome trials. Lancet Diabetes Endocrinol. 2019;7(10):776-85.

38. Sehested TSG, Bjerre J, Ku S, Chang A, Jahansouz A, Owens DK, et al. Cost-effectiveness of Canakinumab for Prevention of Recurrent Cardiovascular Events. JAMA Cardiol. 2019;4(2):12835.

39. Stam-Slob MC, van der Graaf Y, de Boer A, Greving JP, Visseren FLJ. Cost-effectiveness of PCSK9 inhibition in addition to standard lipid-lowering therapy in patients at high risk for vascular disease. Int J Cardiol. 2018;253:148-54.

40. Kaasenbrood L, Boekholdt SM, van der Graaf Y, Ray KK, Peters RJG, Kastelein JJP, et al. Distribution of Estimated 10-Year Risk of Recurrent Vascular Events and Residual Risk in a Secondary Prevention Population. Circulation. 2016;134(19):1419-29.

41. Kaasenbrood L, Poulter NR, Sever PS, Colhoun HM, Livingstone SJ, Boekholdt SM, et al. Development and Validation of a Model to Predict Absolute Vascular Risk Reduction by Moderate-Intensity Statin Therapy in Individual Patients With Type 2 Diabetes Mellitus: The Anglo Scandinavian Cardiac Outcomes Trial, Antihypertensive and Lipid-Lowering. Circ Cardiovasc Qual Outcomes. 2016;9(3):213-21.

42. Dorresteijn JAN, Visseren FLJ, Wassink AMJ, Gondrie MJA, Steyerberg EW, Ridker PM, et al. Development and validation of a prediction rule for recurrent vascular events based on a cohort study of patients with arterial disease: the SMART risk score. Heart. 2013;99(12):866-72. 
43. Bohula EA, Bonaca MP, Braunwald E, Aylward PE, Corbalan R, De Ferrari GM, et al. Atherothrombotic Risk Stratification and the Efficacy and Safety of Vorapaxar in Patients With Stable Ischemic Heart Disease and Previous Myocardial Infarction. Circulation. 2016;134(4):30413.

44. Kengne AP, Patel A, Marre M, Travert F, Lievre M, Zoungas S, et al. Contemporary model for cardiovascular risk prediction in people with type 2 diabetes. Eur J Cardiovasc Prev Rehabil. 2011;18(3):393-8.

45. Stevens RJ, Kothari V, Adler Al, Stratton IM. The UKPDS risk engine: a model for the risk of coronary heart disease in Type II diabetes (UKPDS 56). Clin Sci. 2001;101(6):671-9.

46. Cooney MT, Selmer R, Lindman A, Tverdal A, Menotti A, Thomsen T, et al. Cardiovascular risk estimation in older persons: SCORE O.P. Eur J Prev Cardiol. 2016;23(10):1093-103.

47. Stam-Slob MC, Visseren FLJ, Jukema J, van der Graaf Y, Poulter NR, Gupta A, et al. Personalized absolute benefit of statin treatment for primary or secondary prevention of vascular disease in individual elderly patients. Clin Res Cardiol. 2017;106(1):58-68.

48. Kannel WB, D'Agostino RB. The Importance of Cardiovascular Risk Factors in the Elderly. Am J Geriatr Cardiol. 1995;4(2):10-23.

49. Berry SD, Ngo L, Samelson EJ, Kiel DP. Competing risk of death: an important consideration in studies of older adults. J Am Geriatr Soc. 2010;58(4):783-7.

50. Joint British Societies' consensus recommendations for the prevention of cardiovascular disease (JBS3). Heart. 2014;100(Suppl 2):ii1 LP-ii67.

51. Jaspers NEM, Blaha MJ, Matsushita K, van der Schouw YT, Wareham NJ, Khaw K-T, et al. Prediction of individualized lifetime benefit from cholesterol lowering, blood pressure lowering, antithrombotic therapy, and smoking cessation in apparently healthy people. Eur Heart J. 2019;31:1-10.

52. Kaasenbrood L, Bhatt DL, Dorresteijn JAN, Wilson PWF, D'Agostino RB, Massaro JM, et al. Estimated life expectancy without recurrent cardiovascular events in patients with vascular disease: The SMART-REACH model. J Am Heart Assoc. 2018;7(16).

53. Berkelmans GF, Gudbjörnsdottir S, Visseren FL, Wild SH, Franzen S, Chalmers J, et al. Prediction of individual life-years gained without cardiovascular events from lipid, blood pressure, glucose, and aspirin treatment based on data of more than 500000 patients with Type 2 diabetes mellitus. Eur Heart J. $2019 ; 40(34): 2899-2906$.

54. Dorresteijn JAN, Visseren FLJ, Ridker PM, Wassink AMJ, Paynter NP, Steyerberg EW, et al. Estimating treatment effects for individual patients based on the results of randomised clinical trials. BMJ. 2011;343:d5888.

55. Dorresteijn JAN, Kaasenbrood L, Cook NR, van Kruijsdijk RCM, van der Graaf Y, Visseren FLJ, et al. How to translate clinical trial results into gain in healthy life expectancy for individual patients. BMJ. 2016;352:i1548.

56. van der Leeuw J, Ridker PM, van der Graaf Y, Visseren FLJ. Personalized cardiovascular disease prevention by applying individualized prediction of treatment effects. Eur Heart J. 
2014;35(13):837-43.

57. van der Leeuw J, Visseren FLJ, Woodward M, van der Graaf Y, Grobbee DE, Harrap S, et al. Estimation of individual beneficial and adverse effects of intensive glucose control for patients with type 2 diabetes. Diabetologia. 2016;59(12):2603-12.

58. Stam-Slob MC, Connolly SJ, van der Graaf Y, Leeuw J van der, Dorresteijn JA, Eikelboom JW, et al. Individual Treatment Effect Estimation of 2 Doses of Dabigatran on Stroke and Major Bleeding in Atrial Fibrillation. Circulation. 2019;139(25):2846-2856.

59. Jaspers NEM, Visseren FLJ, Numans ME, Smulders YM, van Loenen Martinet FA, van der Graaf $Y$, et al. Variation in minimum desired cardiovascular disease-free longevity benefit from statin and antihypertensive medications: a cross-sectional study of patient and primary care physician perspectives. BMJ Open. 2018;8(5):e021309.

60. Kent DM, Steyerberg E, van Klaveren D. Personalized evidence based medicine: predictive approaches to heterogeneous treatment effects. BMJ. 2018;363:k4245.

61. Yusuf S, Wittes J, Probstfield J, Tyroler HA. Analysis and interpretation of treatment effects in subgroups of patients in randomized clinical trials. JAMA. 1991;266(1):93-8.

62. van Klaveren D, Vergouwe Y, Farooq V, Serruys PW, Steyerberg EW. Estimates of absolute treatment benefit for individual patients required careful modeling of statistical interactions. J Clin Epidemiol. 2015;68(11):1366-74.

63. The Look AHEAD Research Group, Wing RR, Bolin P, Brancati FL, Bray GA, Clark JM, et al. Cardiovascular Effects of Intensive Lifestyle Intervention in Type 2 Diabetes. N Engl J Med. 2013;369(2):145-54

64. Davies MJ, D'Alessio DA, Fradkin J, Kernan WN, Mathieu C, Mingrone G, et al. Management of hyperglycaemia in type 2 diabetes, 2018. A consensus report by the American Diabetes Association (ADA) and the European Association for the Study of Diabetes (EASD). Diabetologia. 2018;61(12):2461-98.

65. 4. Lifestyle Management: Standards of Medical Care in Diabetes-2018. Diabetes Care. 2018;41(Suppl 1):S38-50.

66. Grisolia JM, Longo A, Hutchinson G, Kee F. Comparing mortality risk reduction, life expectancy gains, and probability of achieving full life span, as alternatives for presenting CVD mortality risk reduction: A discrete choice study of framing risk and health behaviour change. Soc Sci Med. 2018;211:164-74.

67. Jegan NRA, Kürwitz SA, Kramer LK, Heinzel-Gutenbrunner M, Adarkwah CC, Popert U, et al. The effect of a new lifetime-cardiovascular-risk display on patients' motivation to participate in shared decision-making. BMC Fam Pract. 2018;19(1):1-9.

68. Ference BA, Bhatt DL, Catapano AL, Packard CJ, Graham I, Kaptoge S, et al. Association of Genetic Variants Related to Combined Exposure to Lower Low-Density Lipoproteins and Lower Systolic Blood Pressure with Lifetime Risk of Cardiovascular Disease. J Am Med Assoc. 2019;322(14):1381-91. 
CHAPTER 10 | General discussion 


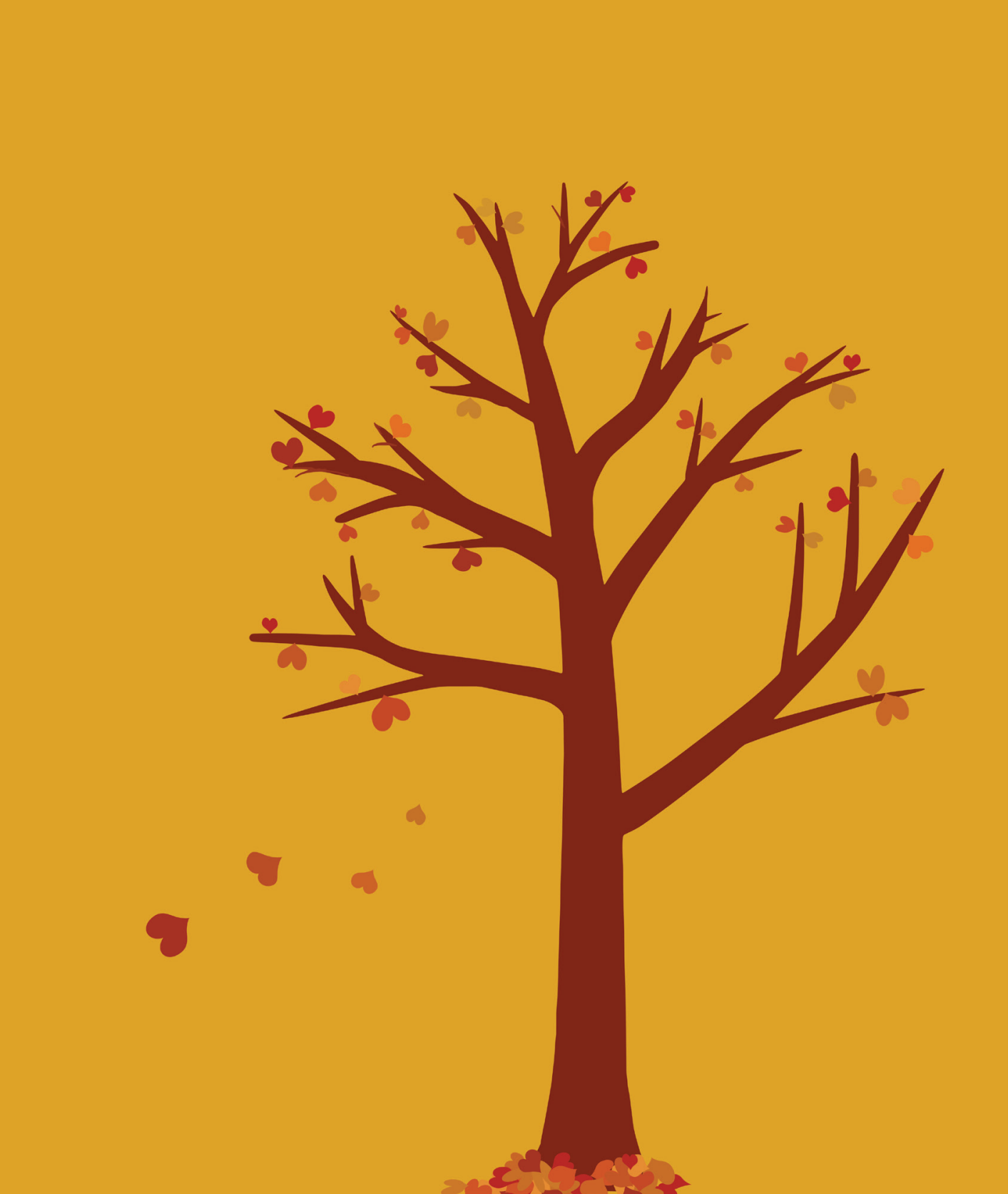




\section{APPENDIX}

Summary

Samenvatting (voor niet-ingewijden)

Contributing authors

Dankwoord

List of publications

Curriculum Vitae 


\section{SUMMARY}

Despite many international efforts to reduce the disease burden, cardiovascular disease (CVD) remains the most important cause of mortality worldwide. In international guidelines, cardiovascular risk management is the cornerstone in CVD prevention, consisting of treatment of modifiable risk factors for CVD, including lipid lowering, blood pressure lowering, blood glucose lowering (in patients with diabetes), and smoking cessation. As these treatments are associated with a high burden on the health care system globally, it is important to be able to identify those patients who will benefit most from preventive treatment strategies.

This thesis consists of two parts: Part $\mathbf{1}$ focuses on traditional and novel risk factors for CVD and type 2 diabetes, while Part 2 focuses on the prediction of CVD risk and treatment effects for individual patients.

\section{Part 1: Risk factors for cardiovascular disease and type 2 diabetes}

Secondary prevention remains an important part of the treatment of patients with established CVD. However, despite increasingly intensified treatment targets and novel treatment options, patients still experience recurrent CVD, a concept dubbed 'residual (vascular) risk'.

In Chapter 2, we have demonstrated that in patients who have recently had a first manifestation of CVD, classical risk factors such as smoking, lipids, and high blood pressure, are not only important risk factors for the first recurrent (subsequent) atherosclerotic event, but for multiple events in the future, even well into long-term follow-up (hazard ratio (HR) for cumulative events 1.26 [95\% confidence interval [Cl] 1.17 - 1.35] for smoking; 1.09 [95\%Cl 1.06 - 1.12] for non-HDL-cholesterol; 1.04 [95\% Cl 1.03 - 1.06] per $10 \mathrm{mmHg}$ for systolic blood pressure [SBP]). Recurrent event analysis captures the full cumulative burden of CVD in patients and does not merely focus upon the first subsequent cardiovascular event. These data thus confirm the importance of these classical risk factors for the total cardiovascular burden in patients with established CVD. The results of this study underline the necessity of adequate treatment of traditional risk factors in patients with CVD.

But even patients in whom the classical risk factors have been treated appropriately, a residual risk remains. A plethora of biomarkers have been studied in relation to the risk of CVD. These can be causally related risk factors involved in atherosclerosis, but noncausally related biomarkers can also be of clinical importance if they can improve CVD risk stratification to improve clinical decision making. In Chapters $\mathbf{3}$ and 4, we have studied one such potential risk factor for diabetes and CVD: thyroid function measured as the level of thyroid stimulating hormone (TSH). There is evidence in the medical literature that 
even within the established reference range, the level of TSH may be related to the risk of morbidity and mortality. In Chapter 3, we have performed a cohort study and metaanalysis, both showing no evidence that TSH levels within the normal range are related to the risk of incident type 2 diabetes (pooled HR 1.06; 95\% Cl 0.99-1.14 in the meta-analysis). In Chapter 4, we have demonstrated that these same reference range TSH levels are inversely related to the risk of future stroke (HR per mIU/L 0.64, 95\% Cl 0.45-0.89), but not to the risk of other cardiovascular events (HR for major cardiovascular events [MACE] 0.93; $95 \% \mathrm{Cl}$ 0.80-1.08). Despite the evidence that thyroid function is a risk factor for CVD, even within the normal range, there is of yet no evidence that thyroid hormone replacement has important health benefits outside of overt thyroid dysfunction.

\section{Part 2: Individualized prediction of CVD risk and treatment effects}

Current international guidelines advise risk factor treatment based on the assumed or predicted future risk of new cardiovascular events, under the principle, "the higher the risk, the higher the benefit". In current international guidelines, including the guidelines by the European Society of Cardiology (ESC) and American College of Cardiology/American Heart Association (ACC/AHA), patients with established CVD or with diabetes mellitus are all assumed to be at (very) high risk of future CVD. For patients without established CVD and diabetes, risk prediction models are recommended to determine what the future CVD risk for an individual patient is, and to treat accordingly. These include the Systematic COronary Risk Evaluation (SCORE) model in the ESC guidelines, and the atherosclerotic cardiovascular disease (ASCVD) model in the ACC/AHA guidelines.

\section{Going from risk to treatment effect estimations}

The main goal of risk prediction in clinical practice, is to be able to make informed treatment decisions for individual patients to lower their (residual) risk of future CVD. Under the principle "the higher risk, the higher the benefit", estimations of future risk can be used to decide which patients should be treated the most strictly. This principle is based on the following assumption: if the relative risk reduction (RRR) from a preventive therapy is the same for all patients, those patients with the highest risk of CVD, will have the highest absolute risk reduction (ARR). For example, a RRR of $20 \%$ for an intervention (e.g. from $10 \mathrm{mmHg}$ SBP lowering), will lead to a $2 \%$ 10-year ARR in a patient with a 10 year CVD risk of $10 \%$, but to a 10\% 10-year ARR in a patient with a 10 -year risk of $50 \%$. These calculations are made under the assumption that the RRR is indeed uniform across a patient population.

One way to present data on whether RRR is uniform across patients populations is to perform subgroup analyses, e.g. in men versus women, younger versus older patients, or 
patients with and without diabetes mellitus, in randomized controlled trials (RCTs) However, traditional subgroup analyses have several disadvantages. Firstly, limited statistical power leads to both false positive and false negative subgroup effects. Furthermore, only one patient characteristic is tested at a time, while a real-life patient consists of many different characteristics (e.g. an older man with diabetes versus a younger woman without diabetes). In Chapter 5, we described and illustrated a method to test the assumption that RRR is uniform across a patient population from an RCT by testing whether the RRR differs across levels of estimated baseline (i.e. untreated) risk using multivariate risk prediction models. Heterogeneity of treatment effect can be assessed in RCTs using Cox models of trial allocation, baseline risk as predicted with a multivariate risk model, and their interaction term as covariates. In the example included in the chapter of the Randomized Evaluation of Long Term Anticoagulant Therapy (RE-LY) trial, heterogeneity of the relative treatment effect existed for the major bleeding outcome. There was significant interaction between baseline risk and treatment effect from dabigatran versus warfarin in RE-LY $(p<0.001)$ on the major bleeding endpoint. Quartile-specific HRs for major bleeding ranged from $0.40(95 \% \mathrm{Cl} 0.26-0.61)$ to $1.04(95 \% \mathrm{Cl} 0.83-1.03)$ for dabigatran in a dose of $110 \mathrm{mg}$ and from $0.61(95 \% \mathrm{Cl} 0.42-0.88)$ to $1.20(95 \% \mathrm{Cl} 0.97-1.50)$ for dabigatran in a dose of $150 \mathrm{mg}$ compared to warfarin. There was however no evidence of heterogeneity of the treatment effect on the primary trial outcome $(p=0.71)$. The consequence of this finding is that the single hazard ratio for major bleeding reported by a trial cannot be applied to all patients, and a more individualized approach is warranted. Although RRR may be uniform across a patient population seen from the perspective of efficacy, this may not be the case for safety.

\section{Risk and treatment effect predictions can be performed in different patient populations}

Although current international guidelines only recommend risk prediction for younger and middle-aged 'apparently healthy persons', 10-year risk estimation tools exist for many different patient populations, for example, patients with established CVD (SMART or TRS $2^{\circ} \mathrm{P}$ ), patients with type 2 diabetes (ADVANCE or UKPDS), and older persons (SCORE O.P. or the PROSPER older person-specific risk model). Prediction models for specific populations may include predictors that are specifically aimed these populations. Although current guidelines assume all patients with CVD or diabetes to be at (very) high risk, it has been found that this view is rather too simplistic. Risk prediction may have an important place for these patients too, especially to identify patients with a high residual risk of CVD after the general prevention goals are met.

For older persons, it is important to take the competing risk of non-CVD mortality into account when predicting CVD risk. Failing to adjust for competing risks will lead to overestimations of CVD risk in older persons, in whom the competing risks become 
increasingly more important. In Chapter 6, we have validated a previously developed older person-specific risk model, derived in the PROSPER trial, in several patient populations. This risk model reliable estimates 10-year CVD risk in older persons from several geographical backgrounds, with good calibration and a pooled C-statistic of 0.68 (95\% Cl 0.67-0.69). Furthermore, we have included an example of how this model can be used to predict treatment effects from cardiovascular risk management in older persons by combining risk predictions with results from RCTs or meta-analyses. These treatment effect estimations can be used for shared-decision making in clinical practice. We have demonstrated that there is a wide range in ARR from blood pressure lowering between older persons (median ARR 8.0\%; interquartile range 3.0-12.9\%).

\section{Risk and treatment effect predictions can be performed for different time perspectives}

'Traditional' risk prediction models estimate the risk in the next few years, for example the 1-year risk, 5-year risk, or 10-year risk of CVD, expressed as a percentage (e.g. "10\% risk of fatal CVD in the next 10 years"). The associated treatment effect predictions, ARRs, are also expressed as a percentage. A novel method to look at future CVD risk uses a lifetime perspective, and is expressed as the median "CVD-free life expectancy ". Combining these lifetime predictions with relative treatment effects from trials or meta-analyses does not give a percentage absolute risk reduction, but rather "months (or years) CVD-free life expectancy gained", which is more intuitive to understand for patients in clinical practice. The 10-year absolute risk is very dependent on age: The higher the age, the higher the risk. However, lifetime treatment effects lessen with increasing age, simply because the remaining life expectancy is relatively limited. In Chapter 7, we have shown that using lifetime treatment effect estimations from preventive strategies, rather than the traditional 10-year absolute risk, to make treatment decisions, will lead to initiation of treatment in younger patients with high risk factor levels, rather than older patients, as is currently the case. Thus, lifetime predictions have the potential to cause a proverbial paradigm shift from treating the patients with the highest risk, to treating the patients with the highest benefit.

Lifetime estimations can be performed in several different patient populations, including 'apparently healthy persons' (LIFE-CVD model), patients with diabetes (DIAL model) and patients with established CVD (SMART-REACH model). In Chapter 8 we have estimated the increase in life expectancy free from CVD when adding a low-dose direct oral anticoagulant (DOAC) to aspirin in individual patients with established CVD from the COMPASS trial using the SMART-REACH model. We further developed a new lifetime model to predict the life expectancy free from major bleeding and the loss in life expectancy free from major bleeding when adding a low-dose DOAC to aspirin in these patients. We demonstrated that in the majority of patients, the increase in CVD- 
free life expectancy outweighs the decrease in major bleeding-free life expectancy, a very comforting finding. Using these lifetime models, benefits and bleeding risk can be weighed for each individual patient, which could facilitate treatment decisions in clinical practice. Cost-effectiveness analyses and discussions between patients, doctors, and health policy makers are necessary to establish what is sufficient lifetime benefit from medication to warrant preventive treatment.

\section{Direct treatment effect estimations}

In the previously discussed chapters, treatment effect estimations were estimated by combining a single relative treatment effect with multivariate risk predictions. In cases where the assumption of a uniform RRR is not met, it is also possible to directly estimate treatment effect using a multivariate risk model derived in an RCT. This method is an alternative method to assess heterogeneity of the RRR from the one presented in Chapter 5. A multivariate prediction model including treatment-by-covariate interaction terms for all relevant predictors can be derived in an RCT. In Chapter 9, we derived such a model in the Look AHEAD trial, a trial studying an intensive lifestyle intervention aimed at weight loss in overweight and obese patients with type 2 diabetes. In the primary analysis of this trial, no significant treatment effect was found on the risk of CVD. A prediction model including all possible treatment allocation-by-covariate interaction terms was derived in the trial study population. After stratification of the trial population in quartiles of predicted treatment effect, it was shown that there was a quartile of patients with a high observed benefit from the lifestyle intervention ( $\mathrm{HR} 0.64 ; 95 \% \mathrm{Cl} 0.49-0.83$ ), but also a quartile of patients with a detrimental effect from the intervention ( $\mathrm{HR} 1.37,95 \% \mathrm{Cl} 1.09-1.73)$. This is very interesting and clinically important, as it has always been assumed that lifestyle interventions are relatively harmless, and are recommended for all patients with type 2 diabetes. We would recommend that the assessment of differences in relative treatment effect among patients is routinely included in RCT design, using the methods from either Chapter $\mathbf{5}$ or Chapter $\mathbf{9}$.

\section{Concluding remarks}

Although more research is needed in the fields of prognostication in cardiovascular research, it is clear from recent developments that there is a shift towards a more individualized approach to cardiovascular prevention in many different patient categories. Methodological advancements allow a shift from a risk-based approach with treatment decision thresholds based on 10-year risk, towards a benefit-based approach in which we can estimate the treatment effects from preventive medication for individual patients, allowing us to choose the right therapy for the right patient. 
APPENDIX | Summary

$\mathbf{A}$

233 


\section{SAMENVATTING (voor niet-ingewijden)}

Hart- en vaatziekten blijven wereldwijd de belangrijkste oorzaak van ziekte en sterfte. Preventieve maatregelen richten zich op behandelbare risicofactoren, waaronder stoppen met roken, cholesterolverlaging, bloeddrukverlaging, bloedverdunnende middelen en het verlagen van de bloedglucose (bij patiënten met diabetes). Aangezien deze behandelingen wereldwijd voor een grote belasting van het gezondheidszorgsysteem vormen, met hoge bijbehorende kosten, is het belangrijk om de patiënten te identificeren die het meest baat zullen hebben bij preventieve behandelstrategieën. Daarvoor moeten onderzoeksresultaten die op groepsniveau worden gepresenteerd, vertaald worden naar individuele patiënten.

Dit proefschrift bestaat uit twee delen: Deel 1 richt zich op risicofactoren voor hart- en vaatziekten en type 2 diabetes; Deel 2 gaat over het voorspellen van het risico op hart- en vaatziekten en het voorspellen van behandeleffecten voor individuele patiënten.

\section{Deel 1: Risicofactoren voor hart- en vaatziekten en diabetes type 2}

Al enkele decennia maakt secundaire preventie een belangrijk deel uit van de behandelingsstrategie bij patiënten die hart- en vaatziekten hebben ontwikkeld, zoals patiënten die een hartinfarct of beroerte hebben doorgemaakt, of een vaatvernauwing in de benen hebben. Ondanks dat we risicofactoren over de jaren heen steeds intensiever zijn gaan behandelen, hebben deze patiënten echter nog steeds een risico om opnieuw een vaatprobleem door te maken. In Hoofdstuk 2 hebben we aangetoond dat bij patiënten die recentelijk een eerste manifestatie van hart- en vaatziekten hebben gehad, klassieke risicofactoren zoals roken, cholesterol en hoge bloeddruk niet alleen belangrijke risicofactoren zijn voor het eerste daarop volgende vaatprobleem, maar voor meerdere toekomstige vaatproblemen (bijvoorbeeld meerdere hartinfarcten of meerdere dotterbehandelingen) in de toekomst, zelfs tot vele jaren later. Deze data bevestigen het belang van deze klassieke risicofactoren voor de totale cardiovasculaire belasting bij patiënten met een voorgeschiedenis van hart- en vaatziekten. Deze risicofactoren blijven dus ook belangrijk als je al hart- en vaatziekten hebt ontwikkeld.

Maar zelfs bij patiënten bij wie de 'klassieke' risicofactoren op de juiste wijze zijn behandeld, is er nog steeds een zogenaamd 'residueel risico' op toekomstige vaatproblemen. In Hoofdstukken $\mathbf{3}$ en $\mathbf{4}$ hebben we een nieuwe potentiële risicofactor voor type 2 diabetes en hart- en vaatziekten bestudeerd, namelijk de schildklierfunctie. Er zijn aanwijzingen in de literatuur dat zelfs binnen de referentiewaarden de hoogte van het schildklier-stimulerend hormoon (TSH; het hormoon dat de schildklier aanzet tot de 
productie van schildklierhormoon), gerelateerd kan zijn aan het risico op bepaalde ziekten.

In Hoofdstuk $\mathbf{3}$ hebben we laten zien dat TSH binnen de referentiewaarden geen relatie lijkt te hebben met het risico op het ontstaan van diabetes type 2. In Hoofdstuk 4 hebben we aangetoond dat de hoogte van het TSH binnen de referentiewaarden wel omgekeerd evenredig is met het risico op een toekomstige beroerte, maar niet met het risico op andere hart- en vaatziekten. Ondanks het bewijs dat de schildklierfunctie een risicofactor is voor hart- en vaatziekten, is er echter nog geen bewijs dat schildklierhormoonvervanging belangrijke gezondheidsvoordelen heeft in mensen zonder schildklierafwijkingen.

\section{Deel 2: Geïndividualiseerde voorspellingen van het risico op hart- en vaatziekten en behandeleffecten}

In de preventie van hart- en vaatziekten wordt in de huidige internationale richtlijnen onderscheid gemaakt tussen mensen met een voorgeschiedenis van hart- en vaatziekten, mensen met type 2 diabetes en mensen zonder vaatziekten of type 2 diabetes. Patiënten met hart- en vaatziekten of met type 2 diabetes worden allemaal verondersteld een (zeer) hoog risico te lopen op toekomstige vaatproblemen. Voor patiënten zonder vaatziekten of diabetes worden momenteel al risicomodellen gebruikt om patiënten te identificeren die een hoog risico lopen op hart- en vaatziekten en dus mogelijk meer baat zullen hebben bij preventieve maatregelen.

Het belangrijkste doel van risicovoorspelling in de klinische praktijk is dus om geïnformeerde beslissingen te kunnen nemen over de behandeling van individuele patiënten om hun (residueel) risico op toekomstige hart- en vaatziekten te verlagen.

Er wordt verondersteld dat hoe hoger het risico van een persoon om hart- en vaatziekten te krijgen is, hoe hoger het te verwachten effect van behandeling is. Als de relatieve risicovermindering van een bepaalde preventieve therapie voor alle patiënten gelijk is, zullen de patiënten met het hoogste risico op hart- en vaatziekten immers de hoogste absolute risicovermindering hebben. Wiskundig gezien is dit logisch: als een bepaalde behandeling het risico op een vaatprobleem met $20 \%$ zal verminderen, zal iemand die een 10 -jaars risico van $10 \%$ op een vaatprobleem heeft, een risicoreductie van $2 \%$ hebben (20\% van 10\%), terwijl iemand die een 10 -jaarsrisico van $50 \%$ heeft, een risicoreductie van $10 \%$ zal hebben (20\% van $50 \%$ ). In het eerste geval moet je 50 patiënten behandelen gedurende 10 jaar om 1 keer hart- en vaatziekten te voorkomen terwijl in het tweede geval dit maar 10 patiënten is.

Deze berekeningen kunnen worden gemaakt in de veronderstelling dat de relatieve risicoreductie inderdaad voor alle patiënten gelijk is. In veel medicatiestudies is het al gebruikelijk om te testen of deze relatieve reductie hetzelfde is in verschillende 
subgroepen van patiënten, bijvoorbeeld bij mannen versus vrouwen, jongere versus oudere patiënten, of patiënten met en zonder diabetes mellitus. Deze methode heeft echter een aantal nadelen. Een belangrijk nadeel is dat er slechts één patiëntkenmerk tegelijk wordt getest, terwijl een echte patiënt(e) uit veel verschillende kenmerken bestaat (bijvoorbeeld een oudere man met diabetes versus een jongere vrouw zonder diabetes). In Hoofdstuk 5 hebben we een methode beschreven en geïllustreerd om deze aanname te testen in medicijnstudies op basis van meerdere patiëntkarakteristieken tegelijk. Dit wordt gedaan door patiënten in te delen op basis van hun voorspelde risico op hart- en vaatziekten en dan te kijken of de relatieve risicoreductie van de bestudeerde behandeling voor alle niveaus van risico vergelijkbaar is. Eén van de voorbeelden die wordt getoond gaat over een medicatiestudie waarbij twee type bloedverdunners worden vergeleken om bij patiënten met boezemfibrilleren het risico op beroerte en vaatverstoppingen te verkleinen. Een nadeel van bloedverdunners is dat ze het risico op bloedingen vergroten. In dit hoofdstuk laten we zien dat bij patiënten met een laag risico om te bloeden, dabigatran het risico op bloeden verlaagt ten opzichte van warfarine, terwijl in patiënten met een hoog risico op bloeden, het risico op bloeden vergelijkbaar is voor de twee middelen of zelfs iets hoger is bij een hogere dosering van dabigatran vergeleken met warfarine. Het relatieve behandeleffect op het eindpunt beroerte en vaatverstoppingen is wel vergelijkbaar voor alle niveaus van risico. De resultaten uit dit hoofdstuk laten dus zien dat de gemiddelde behandeleffecten die gerapporteerd zijn voor deze medicatiestudie niet voor alle patiënten waar zijn, wat zeer belangrijk is bij het nemen van beslissingen over de behandeling in de klinische praktijk.

\section{Risico- en behandeleffectvoorspellingen kunnen in diverse groepen patiënten worden gemaakt}

Hoewel er in de internationale richtlijnen op dit moment alleen voor patiënten zonder harten vaatziekten en diabetes wordt aangeraden om risicomodellen te gebruiken, bestaan er risicomodellen voor veel meer groepen patiënten: bijvoorbeeld voor patiënten met een voorgeschiedenis van hart- en vaatziekten, voor mensen met diabetes en specifiek voor oudere mensen ongeacht de voorgeschiedenis. Het gedachtegoed dat alle patiënten met hart- en vaatziekten en/of diabetes automatisch een hoog risico hebben op toekomstige vaatproblemen blijkt te eenvoudig te zijn. Zeker als de 'basispreventie' (stoppen met roken, bloeddrukverlaging, cholesterolverlaging) al goed is ingezet, blijkt er een groot verschil te zijn in het residueel risico (het risico wat je overhoudt na behandeling) op toekomstige vaatproblemen.

Voorspellingsmodellen voor specifieke patiëntengroepen kunnen risicofactoren bevatten die specifiek voor die patiëntengroep belangrijk is. Als voorbeeld: bij ouderen is het risico op andere soorten ziekten (anders dan hart- en vaatziekten, bijvoorbeeld kanker) veel groter dan bij jongere mensen en die risico's hebben ook invloed op het risico van een 
persoon om hart- en vaatziekten te krijgen. Immers, cru gezegd, als een patient een grote kans heeft om te overlijden aan bijvoorbeeld kanker, dan is de kans om te overlijden aan hart- en vaatziekten natuurlijk lager. Als hier geen rekening mee gehouden wordt, dan overschatten we consequent de kans op hart- en vaatziekten, en zoals we eerder bespraken, de potentiele winst van behandeling. In Hoofdstuk 6 hebben we een risicomodel onderzocht dat speciaal ontwikkeld is om het risico op hart- en vaatziekten te voorspellen in ouderen. We hebben laten zien dat het risicomodel betrouwbare risicovoorspellingen geeft voor patiënten die ouder dan 65 jaar zijn. Verder hebben we laten zien hoe dit model kan worden gebruikt om behandelingseffecten van preventieve behandeling bij ouderen te voorspellen, in dit geval bloeddrukverlaging, door de risicovoorspellingen te combineren met de relatieve behandeleffecten uit een medicatiestudie. Zoals verwacht is er een groot verschil in de te behalen winst van bloeddrukverlaging in oudere mensen. Deze voorspellingen kunnen worden gebruikt om behandelbeslissingen te nemen voor artsen samen met hun patiënt in de klinische praktijk.

\section{Risico- en behandeleffectvoorspellingen kunnen voor diverse tijdsduren worden gemaakt}

'Traditionele' risicovoorspellingsmodellen schatten het risico in de komende aantal jaren, bijvoorbeeld het 1-jaarsrisico, het 5-jaarsrisico of het 10-jaarsrisico op hart- en vaatziekten, uitgedrukt als een percentage (bijvoorbeeld " $5 \%$ 10-jaarsrisico op fatale hart- en vaatziekten"). Schattingen van het behandeleffect van deze modellen geven een absolute risicoreductie, ook uitgedrukt in een percentage. Een nieuwe methode om naar het risico op toekomstige hart- en vaatziekten te kijken, maakt gebruik van een levenslang perspectief en wordt uitgedrukt als de mediane "hart- en vaatziektevrije levensverwachting". Als we op basis van deze 'levenslange' voorspellingen de individuele behandeleffecten willen berekenen, krijgen we geen procentuele absolute risicovermindering, maar de "te winnen maanden (of jaren) hart- en vaatziektevrije levensverwachting", wat intuïtiever is om te begrijpen voor patiënten dan een percentage.

De hoogte van het 10-jaarsrisico wordt met name bepaald door de leeftijd van een patiënt: hoe ouder, hoe hoger het risico. Het aantal te winnen hart- en vaatziektevrije jaren wordt juist minder bij hogere leeftijd, simpelweg omdat de overgebleven levensverwachting ook minder wordt. In Hoofdstuk $\mathbf{7}$ laten we zien dat het gebruik van behandeleffectvoorspellingen in een levenslang perspectief om beslissingen over de behandeling te nemen zal leiden tot het starten van behandeling bij jongere patiënten met belangrijke risicofactoren, in plaats van bij oudere patiënten met een hoog 10-jaarsrisico, zoals nu het geval is. Dit zou tot een heel grote verandering kunnen leiden ten opzichte van de huidige situatie, waarin ouderen vaker worden behandeld met preventieve medicatie dan jongere mensen. 
Ook de 'levenslange' schattingen kunnen worden uitgevoerd in verschillende groepen patiënten, waaronder patiënten zonder hart- en vaatziekten (LIFE-CVD model), patiënten met diabetes (DIAL-model) en patiënten met een voorgeschiedenis van hart- en vaatziekten (SMART-REACH-model). In Hoofdstuk 8 hebben we met behulp van het SMART-REACH model een schatting gemaakt van de toename van de levensverwachting zonder hart- en vaatziekten bij het toevoegen van een lage dosis van een bepaalde bloedverdunner, rivaroxaban, aan aspirine bij individuele patiënten met hart- en vaatziekten uit de COMPASS studie. Omdat bloedingen de belangrijkste bijwerking zijn van een bloedverdunner, hebben wij hebben een nieuw model ontwikkeld om de "levensverwachting zonder bloedingen" en het "aantal jaren zonder bloedingen te verliezen" te voorspellen bij het starten van rivaroxaban bij deze patiënten. We hebben aangetoond dat bij de meerderheid van de patiënten de toename van de hart- en vaatziektevrije levensverwachting groter is dan de afname van de bloedingsvrije levensverwachting, een zeer geruststellende bevinding. Kosteneffectiviteitsanalyses en discussies tussen patiënten, artsen en gezondheidsbeleidsmakers zijn nodig om vast te stellen wat voldoende levenslange voordelen van medicatie zijn om het starten van een preventieve behandeling te rechtvaardigen.

\section{Direct schatten van behandeleffecten}

In de eerder besproken hoofdstukken werd het behandeleffect voorspeld door risicovoorspellingen te combineren met het relatieve behandeleffect van een behandeling. Zoals eerder besproken, klopt de veronderstelling dat het relatieve behandeleffect voor iedereen waar is niet altijd. In Hoofdstuk 9 gebruiken we een methodologie die kan worden gebruikt om direct het individuele behandeleffect te schatten waarbij deze aanname niet waar hoeft te zijn. Deze methode kan gebruikt worden om een model te maken waarmee het behandeleffect van een behandeling voor individuele patiënten kan worden geschat, maar ook om in eerste instantie te onderzoeken of er verschillen zijn in het relatieve behandeleffect tussen groepen. Het vormt dus gelijk een alternatieve methode vergeleken met Hoofdstuk $\mathbf{5}$ om die veronderstelling te testen in medicatiestudies.

In deze methode wordt voor elke variabele die in een risicomodel wordt gebruikt, ook een interactieterm toegevoegd met de behandeling die onderzocht wordt in de studie. In Hoofdstuk 9 hebben we zo'n model gemaakt in de Look AHEAD studie, een studie die een intensieve leefstijlinterventie gericht op gewichtsverlies bij patiënten met type 2 diabetes met overgewicht en obesitas bestudeerde. In de originele publicatie van de Look AHEAD studie werd niet gevonden dat deze behandeling een positief effect had op het risico op hart- en vaatziekten. In deze studiepopulatie werd een predictiemodel afgeleid waarbij voor alle variabelen ook een interactie met de behandeling werd toegevoegd aan het model. Daarna hebben we de studiepopulatie opgedeeld in vier groepen op basis van het voorspelde risico en toen gekeken wat het geobserveerde behandeleffect was in die 
groepen. Er bleek toen één groep te zijn met patiënten waarin er wél een positief effect was van de intensieve leefstijlinterventie op het risico op hart- en vaatziekten, maar ook een groep waarin juist een schadelijk effect werd waargenomen. Dit is zeer interessant en klinisch belangrijk, omdat altijd is aangenomen dat leefstijlinterventies relatief ongevaarlijk zijn en daarom worden aanbevolen voor alle patiënten met diabetes. Wij bevelen aan om in de opzet van (de statistische methodologie van) een medicatiestudie de formele toetsing van verschillen in het relatieve behandeleffect tussen patiënten altijd op te nemen, met behulp van de methoden uit Hoofdstuk $\mathbf{5}$ of Hoofdstuk 9.

Hoewel er meer onderzoek nodig is op het gebied van risicovoorspelling in onderzoek naar hart- en vaatziekten, blijkt uit recente ontwikkelingen dat er een verschuiving plaatsvindt naar een meer geïndividualiseerde benadering van preventie van hart- en vaatziekten in veel verschillende patiëntcategorieën. De methodologische vooruitgang maakt een verschuiving mogelijk van een op risico gebaseerde aanpak met behandelingsbeslissingsdrempels op basis van 10 jaarsrisico, naar een aanpak waarbij we de behandeleffecten van preventieve medicatie voor individuele patiënten kunnen inschatten, zodat we de juiste therapie voor de juiste patiënt kunnen kiezen. 


\section{List of publications}

de Vries TI, Visseren FLJ. Cardiovascular risk prediction tools made relevant for GPs and patients. Heart. 2020; accepted.

de Vries TI, Eikelboom JW, Bosch J, et al. Estimating individual lifetime benefit and bleeding risk of adding rivaroxaban to aspirin for patients with stable cardiovascular disease: results from the COMPASS trial. Eur Heart J. 2019;40(46):3771-3778a.

de Vries TI, de Valk HW, van der Graaf Y, et al. Normal-range thyroid-stimulating hormone levels and cardiovascular events and mortality in type 2 diabetes. Diabetes Res Clin Pract. 2019;157:107880.

de Vries TI, Dorresteijn JAN, van der Graaf Y, Visseren FLJ, Westerink J. Heterogeneity of Treatment Effects From an Intensive Lifestyle Weight Loss Intervention on Cardiovascular Events in Patients With Type 2 Diabetes: Data From the Look AHEAD Trial. Diabetes Care. 2019;42(10):1988-1994.

van Welzen BJ, de Vries TI, Arends JE, Mudrikova T, Hoepelman AIM. The use of corticosteroids does not influence CD4+ lymphocyte recovery in HIV-infected patients with advanced immunodeficiency. AIDS Care. 2020 Jun;32(6):701-704.

de Vries TI, Kappelle LJ, van der Graaf Y, et al. Thyroid-stimulating hormone levels in the normal range and incident type 2 diabetes mellitus. Acta Diabetol. 2019;56(4):431440 .

de Vries TI, Alsters SI, Kleinendorst L, van Haaften G, van der Zwaag B, Van Haelst MM. Genetische obesitas: nieuwe diagnostische mogelijkheden. Ned Tijdschr Geneeskd. 2017;161(0):D688.

de Vries TI, R Monroe G, van Belzen MJ, van der Lans CA, Savelberg SM, Newman WG et al. Mosaic CREBBP mutation causes overlapping clinical features of Rubinstein-Taybi and Filippi syndromes. Eur J Hum Genet. 2016;24(9):1363-6. 
Monroe GR, Frederix GW, Savelberg SM, de Vries TI, Duran KJ, van der Smagt JJ, et al. Effectiveness of whole-exome sequencing and costs of the traditional diagnostic trajectory in children with intellectual disability. Genet Med. 2016;18(9):949-56.

de Vries TI, van Haelst MM. Ciliary disturbances in syndromal and non-syndromal obesity. J Pediatr Genet. 2014;3(2):79-88. 


\section{Contributing authors}

Marco Alings

Riitta Antikainen

Folkert W. Asselbergs

Nigel Beckett

Scott D Berkowitz

Michael J. Blaha

Gert Jan de Borst

Jackie Bosch

Michiel L. Bots

Maarten-Jan M. Cramer

Jannick A.N. Dorresteijn

Leanne Dyal

John W Eikelboom

Marielle H. Emmelot-Vonk

Keith AA Fox
Department of Cardiology, Amphia Hospital, Breda, the Netherlands

Medical Research Center Oulu, University of Oulu and Oulu City Hospital, Oulu, Finland

Department of Cardiology, University Medical Center Utrecht, Utrecht, the Netherlands; Institute of Cardiovascular Science, Faculty of Population Health Sciences, University College London, and Health Data Research UK and Institute of Health Informatics, University College London, London, United Kingdom Imperial Clinical Trials Unit, Imperial College London, London, United Kingdom

Clinical Development, Thrombosis, Bayer U.S. LLC, Parsippany, NJ, USA

Johns Hopkins University, Baltimore, USA

Department of Vascular Surgery, University Medical Center Utrecht, The Netherlands

Population Health Research Institute, McMaster University and Hamilton Health Sciences, Hamilton, Ontario, Canada

Julius Center for Health Sciences and Primary Care, University Medical Center Utrecht, the Netherlands

Department of Cardiology, University Medical Center Utrecht, The Netherlands

Department of Vascular Medicine, University Medical Center Utrecht, the Netherlands

Population Health Research Institute, McMaster University and Hamilton Health Sciences, Hamilton, Ontario, Canada

Population Health Research Institute, McMaster University and Hamilton Health Sciences, Hamilton, Ontario, Canada

Department of Geriatrics, University Medical Center Utrecht, Utrecht University, The Netherlands

Centre for Cardiovascular Science, University of Edinburgh, Edinburgh, United Kingdom 
Yolanda van der Graaf

Steven H.J. Hageman

L.J. (Jaap) Kappelle

Kunihiro Matsushita

Majon Muller

Vijay Nambi

Hendrik M. Nathoe

Ron J. Peters

Ruth Peters

Yvo M. Smulders

Manon C. Stam-Slob

Harold W. de Valk

Frank L.J. Visseren

Jan Westerink
Julius Center for Health Sciences and Primary Care, University Medical Center Utrecht, the Netherlands

Department of Vascular Medicine, University Medical Center Utrecht, the Netherlands

Department of Neurology, University Medical Center Utrecht, Utrecht, The Netherlands

Johns Hopkins University, Baltimore, USA

Department of Internal Medicine, VU University, Amsterdam, The Netherlands

Michael E DeBakey Veterans Affairs Hospital and Baylor College of Medicine, Houston, USA

Department of Cardiology, University Medical Center Utrecht, The Netherlands

Department of Internal Medicine, VU University, Amsterdam, The Netherlands

School of Public Health, Imperial College London, London, United Kingdom; Psychology, University of New South Wales, Sydney, Australia; Neuroscience Research Australia, Sydney, Australia

Department of Internal Medicine, VU University, Amsterdam, The Netherlands

Department of Internal Medicine, University Medical Center Utrecht, the Netherlands

Department of Internal Medicine, University Medical Center Utrecht, the Netherlands

Department of Vascular Medicine, University Medical Center Utrecht, the Netherlands

Department of Vascular Medicine, University Medical Center Utrecht, the Netherlands 


\section{Dankwoord}

Het is dan zover! Na inmiddels ruim meer dan 3.5 jaar is mijn proefschrift een feit. Het heeft wat langer op zich doen wachten dan we oorspronkelijk hadden gedacht, maar dan ligt er nu wel een resultaat waar ik enorm trots op ben. Ik wil graag om dit proefschrift af te sluiten nog een hele hoop mensen bedanken.

Prof. dr. F.L.J. Visseren, beste Frank, jouw enthousiasme over het onderzoek en het vakgebied is ontzettend inspirerend. Fantastisch om te zien hoe je met voldoende geloof en volharding de buitenwereld kan overtuigen dat nieuwe ideeën niet altijd slecht hoeven te zijn. Bedankt voor alle steun de afgelopen jaren. Bedankt dat je altijd zo laagdrempelig bereikbaar was en er zo vaak uitgebreid de tijd voor had (of tijd voor nam) om te kunnen sparren. En als laatste ook bedankt dat je me de kans gunde om nog wat langer te blijven om nog deel uit te mogen maken van een aantal fantastische projecten en voor de (op de valreep voor de lockdowns) leuke trip naar Cambridge.

Dr. J. Westerink, beste Jan, bedankt dat je het vertrouwen in mij had om mij bij de Vascu naar binnen te loodsen en ook het vertrouwen dat je gedurende de jaren daarna altijd in mij hebt getoond. Jij hebt vaak net een andere blik op zaken en dat is heerlijk verfrissend. Heel fijn om iemand te hebben die helpt de discussie op gang te brengen. Bij mijn allereerste manuscript mailde je terug: "Natuurlijk heb ik kritiek." en dat is eigenlijk altijd zo gebleven. Ik heb heel veel van je geleerd, niet alleen over onderzoek, maar ook op de polibesprekingen en daarbuiten. Zoals je zelf altijd zegt - de wereld zou saai zijn zonder mensen die een beetje raar zijn!

Verder wil ik graag Prof. dr. Y. van der Graaf en Prof. dr. M. Bots bedanken voor de begeleiding van het researchproject tijdens de master epidemiologie. Beste Yolanda, daarnaast ook bedankt voor de inspirerende begeleiding tijdens de gezamenlijke besprekingen met Nicole.

Dr. J.A.N. Dorresteijn, beste Jannick, ook jou wil ik graag bedanken voor je onmisbare begeleiding, met name op het gebied van predictie. Wat een ontzettend voorrecht om mee te mogen werken aan onderzoek wat echt invloed heeft op de dagelijkse praktijk en ik ben trots op hoe zich dat heeft vertaald naar de prachtige nieuwe U-Prevent website. Ik vind het ontzettend leuk dat je terug bent gekomen naar het UMC en hoop dat je in de toekomst nog heel veel arts-onderzoekers bij de Vascu mag inspireren.

Beste collega's van de vasculaire geneeskunde, beste Wilko, Stan, Melvin, Jorn, Corien en Ilona en Margie, bedankt voor de prettige samenwerking. 
Lieve Inge en Corina, bedankt voor de Nespresso's en de leuke gesprekken. Ik vond het ontzettend gezellig en prettig om met jullie samen te mogen werken op de researchpoli.

Beste UCC-SMART medewerkers; Ursula, Ank, Lies, Loes, Yvonne, Hetty, Baukje en Rutger, bedankt voor de fijne samenwerking bij SMART tijdens mijn jaar als SMART-arts en voor alle harde inzet voor de SMART studie.

De leden van de beoordelingscommissie, Prof. dr. Hoes, Prof. dr. Emmelot-Vonk, Prof. dr. Kaasjager, Prof. dr. Kappelle, Prof. dr. de Koning en Prof. dr. van der Schouw, dank ik voor hun bereidheid dit proefschrift te beoordelen.

I would like to thank all the co-authors for their invaluable contributions to this thesis. In particular, I want to thank Dr. Eikelboom and Dr. Bosch for the opportunity to do research at the PHRI in Hamilton. Dear Jackie, thank you for the warm welcome in Hamilton and for the hospitality at your home. Lucie, thanks for taking me under your wing when I first arrived at PHRI. Katie, thanks for taking me to the mall and to the Mule. Chloe and Elizabeth, thanks for the morning coffee runs. And also Leah, Preston, Liam, Sabrina, Sandra, and Jodi, and others I am surely forgetting, thank you for making my stay in Hamilton a memorable one.

Lieve mede-onderzoekers, lieve "Franks Army", bedankt voor de dagelijkse gezelligheid, eerst op onze kamertjes op de D-vleugel (kamertje 1!) en later in het van Geuns, en achteraf nog mijn welgemeende excuses aan diegenen die wél soms gewoon in stilte hadden willen werken.

Bedankt voor alle mentale steun, brainstorm sessies, R hulp, slechte woordgrappen, memes, Whatsapp-stickers, alle keren dat ik moest huilen van het lachen ("doe de MALE dance!"); de koffietjes bij Micafe, de Kroonluchter of de Pitstop; de uitgebreide lunchsessies (team 100\% pindakaas represent!); de al dan niet uit de hand gelopen (vrijdagmiddag)borrels met té veel biertjes, GTs, wijntjes, kaasstengels, bitterballen en nachos; festivals en (Single)feestjes; en zélfs bedankt voor de (soms mislukte) sportieve uitdagingen (planking challenge, anyone?). Bedankt ook dat jullie de thuiswerk/social distancing periode in het afgelopen jaar toch nog een beetje leuk hebben gehouden met de dagelijkse Zoom-lunches en de digitale VrijMiBo's. Bedankt voor de gezelligheid bij de ESC congressen in München en Parijs. Ik heb echt buikpijn gehad van het gelachen op het 'vriendinnenweekend' in Sevilla (zelfs al hebben we niets van het uitgaansleven meegekregen, oh, en kebab en pizza zijn dus géén tapas) en in Breukelen (featuring dierenprints, ranking de onderzoekers, cocktails, kubben, foute hitjes, Secret Hitler en in het water vallen bij 't suppen). 
Guido, Shahnam, Nicolette en Johanneke, bedankt dat jullie mij zo snel hebben laten thuis voelen bij de Vascu. Gijs, zonder jou had ik hier niet gezeten! Bedankt dat je Jan overtuigde om me op gesprek te laten komen, bedankt voor alle R hulp en voor je heerlijke directheid. Nicole, bedankt voor de lessen over de Oxford komma, je heerlijke humor en je bijzondere recepten. Ik denk nog met veel plezier terug aan de schoenencollectie op kamertje 1. Monique, sportieve, hardwerkende, inspirerende, lieve, empathische collega, zoveel respect voor hoe jij je promotietraject met alle tegenslagen tot zo'n mooi einde hebt weten te brengen. Ik hoop snel weer met je te mogen samenwerken in het Antonius! Cilie, mijn master buddy! Ik heb respect voor de manier waarop je altijd door alle drukte heen tóch gewoon rustig door kon werken (en dan ineens met een scherpe opmerking laten blijken dat je alle onzin stiekem wél gewoon had gehoord). Je was er misschien niet elke borrel bij, maar als je er dan wel was, maakte dat de avond altijd extra gezellig. Jean Paul ("Professor Vendeville"), bedankt voor alle babyfoto's en Game of Thrones en Star Wars-praat. Britt, de hippe Amsterdamse, goedlachs en altijd in voor een borrel. Je bent altijd welkom om bij mij in mijn logeerkamer te komen slapen, als je de laatste trein weer een keertje niet haalt bijvoorbeeld. Steven, bedankt voor alle (R) hulp, flauwe grappen, afleiding tijdens saaie colleges, het samen zeiken kunnen over frustrerende meetings en slechte flowcharts, het aangieren van de borrels, en vooral niet bedankt voor die keer dat ik een fles Smirnoff Ice moest drinken toen ik voor de laatste keer in bus 12 zat. Respect dat je die periode hebt overleefd waarin je de enige man bij de onderzoeksgroep was en verhalen moest aanhoren waar je geld voor over had om het nooit meer te hoeven horen. Helena, mijn favoriete Deense! Gelukkig hebben we je niet afgeschrikt tijdens je wetenschapsstage, want je bent echt een heel erg fijne en leuke collega met een goed gevoel voor humor, al laat je smaak in (Nederlandse) muziek soms een beetje te wensen over. Eline, wat mij betreft een waardig opvolger van Monique. Jammer dat je geen bier lust, maar gelukkig gaat (goede) wijn er wel zéér goed in. Je schaterlach om (flauwe) grappen, over cavia's bijvoorbeeld, was heerlijk om mee te maken. Maria, (bijna) buurvrouw! Ik heb zoveel om jou gelachen in Sevilla en andere momenten, zowel om je humor als om je "flexibele ruggengraat", ik ben blij dat we je naar het van Geuns hebben weten te halen en dat we daar mochten genieten van je (slechte) woordgrappen. Pascal, je kwam in een heel erg gekke periode binnen bij de onderzoeksgroep, maar inmiddels is dat volgens mij helemaal goed gekomen. Een goeie aanwinst voor het predictie team! Marga, we hebben niet lang samengewerkt, maar volgens mij weet jij er héél goed voor te zorgen dat het zeker niet stil gaat vallen in het van Geuns nadat ik weg ben, gezellig om je erbij te hebben! Nadia, eerst ook wel bekend als "de student", welkom terug bij de Vascu. Ik weet zeker dat heel veel plezier gaat hebben in de komende jaren!

Ik vergeet nu natuurlijk nog 1000 dingen, maar ik wil hiermee afsluiten: Lieve collega's, ik ga jullie gelach en gezelligheid op dagelijkse basis missen, maar ik weet zeker dat er nog héél veel uit de hand gelopen borrels gaan volgen. En gelukkig hebben we de foto's nog! 
Verder wil ik graag alle studiemaatjes van de Epi master bedanken met wie ik in die tijd regelmatig $\mathrm{R}$ nerd heb kunnen spelen - waaronder Cilie, Brigitte, Joline, Dirk Jan, Katrien, Aernoud en Bianca. Bedankt voor de koffietjes, borrels, R hulp en in het algemeen voor de gezelligheid tijdens de soms lange weken. Bri, fijn om altijd samen met jou alle exercises in $\mathrm{R}$ te kunnen doen, en bedankt voor de prachtige (date)verhalen.

I would also like to thank my fellow PROUT members for giving me the chance to contribute to something at the UU larger than just my own research project, in particularly my fellow board members Elmar, Federico, Vinzenz and Alice. Hetzelfde geldt voor de MD PhD sensorgroep - ik haalde maandelijks veel voldoening uit onze koffiemeetings!

Daarnaast zijn er nog heel veel mensen die de afgelopen jaren mijn leven heel veel leuker hebben gemaakt. Ik gebruik daarom graag deze zeldzame kans om hen daarvoor te bedanken, dus daar gaan we:

Mijn "vrienden van vroeger": van het Rijnlands, Maaike en Mirjam, Madeleine (lieve roomie!), Tirso; de dreuzeltjes; mijn geneeskunde-vriendinnetjes, Lieke, Isabel, Sabine (mijn andere lieve roomie!)... Ook al is het soms zo dat "Life gets in the way" en het de ene keer wat langer duurt voordat we elkaar weer zien dan de volgende, ben ik heel blij met jullie blijvende aanwezigheid in mijn leven! Al zien we elkaar soms ook heel lang niet, het is altijd direct weer gezellig als vanouds als we elkaar spreken.

Lieve 'burgers', bedankt voor de gezellige weekenden, borrels en barbeques. Clair, bedankt voor het altijd weer uitvoeren van de (on?)dankbare taak van het aangieren van de borrel. En de rest van JC Adonis, bedankt voor de leuke datediners, festivals, en andere borrels en feestjes.

Mijn lieve hockeyteam, bedankt voor een fijne plek om op de donderdagavond (of inmiddels de maandagavond) en zondagen te kunnen ontspannen. Speciale shoutout voor de 'harde kern' van de derde helft voor de vele uren die op de zondagen zijn doorgebracht in het Voordaan clubhuis met een biertje in de hand en een bittergarnituur op tafel.

Lieve jaarclub, Ay Caramba! Lieve Do, Gab, Hans, Juul, Lief, Max, Richt, San, Wub en Yen, wat fantastisch om zo'n leuke groep vriendinnen te mogen hebben, al 10 jaar lang! Heel leuk en eigenlijk ook heel bijzonder om de overgang van de studententijd naar (extreme) burgerlijkheid van iedereen zo dichtbij te kunnen meemaken. Ik weet zeker dat wij samen nog heel veel mooie dingen gaan meemaken. Lieve Sanne, bedankt dat je mij zo vaak hebt gedwongen om te gaan sporten als ik eigenlijk niet wilde;) Lieve Juul, mijn lieve paranimf, ik ben zo blij dat jij bij mij in het clubje zit. Het was altijd al zo fijn om iemand te hebben die mijn verhalen altijd begrijpt, ook al kan de rest daar soms gek van worden ("op de póóóli"). Bedankt voor alle keren duo-clubeten en koffie bij Micafé of in het WKZ. 
Lieve familie de Kleijn, lieve Henk, Lys, Thomas en Moni, Ellen en Oompie, bedankt dat jullie zo'n leuke schoonfamilie zijn waar ik me altijd bij thuis voel. Lieve Louise, jij bent echt fantastisch. Ik ben zo blij dat ik jou tante mag zijn en ik vind het zo leuk om je op te zien groeien en te zien wat een sterke persoonlijkheid jij nu al aan het ontwikkelen bent!

Lieve familie, lieve papa, mama, Kirsten, Laura en Daniël, en Myrthe en Ricardo. Soms zien we elkaar wat vaker, soms wat minder vaak, maar ik weet dat jullie er altijd voor me zijn. Papa en mama, bedankt voor de steun gedurende alle jaren en alle hulp bij het aan de bus werken. Ik ben zo trots om te zien waar de zusjes de Vries nu allemaal terecht zijn gekomen. Kirsten, is ben heel trots om te zien dat je je plekje helemaal hebt gevonden bij de marine, je enthousiasme in je verhalen is aanstekelijk. Superleuk om dit jaar op een fantastische vakantie in Zwitserland te zijn geweest samen. Lieve Laura, wij zijn rond dezelfde tijd gaan werken, maar onze banen en levens zien er nu héél anders uit. Ondanks dat ik nog altijd niet precies snap wat jij nou precies op dagelijkse basis uitvoert (en andersom) weet ik in elk geval dat ik het heel knap wat je doet. Ik vind het superleuk dat jij mijn andere paranimf bent (zelfs al heb je geen idee wat je allemaal staat te wachten). Myrthe, mijn lieve niet-meer-zo-kleine zusje, weet je nog toen wij vorig jaar de WHW met zijn tweeën toch maar even (zo'n beetje) hebben uitgelopen? Good times! Nog steeds heel trots op! Lieve opa en oma, jullie zijn een belangrijke reden geweest dat ik in de vasculaire geneeskunde geïnteresseerd ben geraakt. Ik mis jullie!

Lieve Jasper, bedankt voor je onmisbare steun gedurende de afgelopen jaren, ook als je soms stiekem helemaal geen idee had van waar ik op dagelijkse basis mee bezig was. Ik kon bij jou altijd mijn ei kwijt als ik het moeilijk had of gestressd was en je weet dingen altijd goed te relativeren, iets wat ik soms hard nodig heb als ik teveel in mijn eigen hoofd zit. Bedankt voor de fijne plek om elke dag weer thuis te komen. Ik ben ook heel trots op waar jij in de afgelopen jaren terecht bent gekomen en hoe ik je zie groeien. En ik ben héél benieuwd waar de komende jaren ons gaan brengen (al dan niet in onze camper).

Ik begon mijn proefschrift met een quote van Shakespeare, dus ik vind het niet meer dan passend om daarmee ook af te sluiten:

"I can no other answer make but thanks, And thanks, and ever thanks."

- Shakespeare, Twelfth Night 


\section{Curriculum Vitae}

Tamar de Vries was born on April 3rd, 1992 in Wageningen, the Netherlands. After she graduated cum laude from bilingual education at "het Rijnlands Lyceum" in Oegstgeest in 2010, she studied Medicine at the University of Utrecht. During her Masters programme she was admitted to the Honour's programme, during which she did a research internship at the Department of Clinical Genetics under the supervision of Dr. Mieke van Haelst, studying the genetics of obesity. In 2014, she also spent 3 months on St. Martin for a research internship at the Sister Basilia Residency and Sint

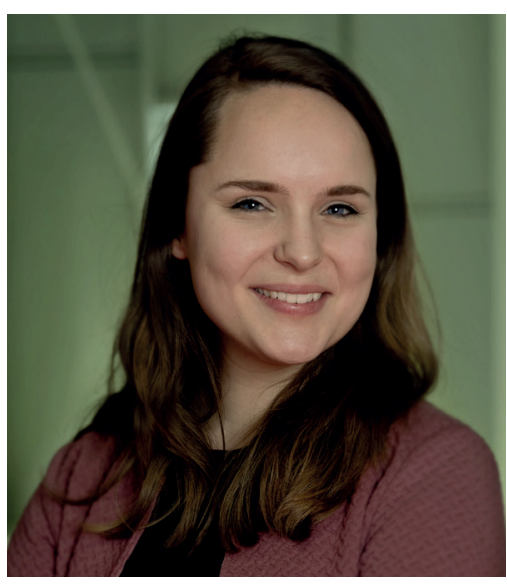
Maarten Medical Center to study the genetics of congenital malformation disorders and intellectual deficit the Dutch West Indies. She obtained her medical degree in 2017 after a senior internship in gastro-enterology and a research internship at the department of infectious diseases. Under the supervision of Jan Westerink and Frank Visseren, she started working on this thesis at the department of Vascular Medicine, University Medical Center Utrecht (UMCU) in February 2017. During her PhD candidacy, Tamar also worked at the Population Health Research Institute in Hamilton, Canada. For the results of this project, presented in Chapter 8 of this thesis, she was nominated for the Young Investigator Award in Thrombosis at the European Society of Cardiology Conference in 2019. She combined her PhD research with the post-graduate master Clinical Epidemiology, from which she graduated in the beginning of 2020. In July 2020, she has started to work as a medical doctor in Internal Medicine at the St. Antonius Hospital in Utrecht. In her free time, Tamar enjoys playing field hockey, playing (board) games, working on her self-converted campervan, travelling, and hiking. 
2

8

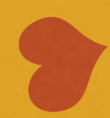

a

8 